\title{
A MALT90 study of the chemical properties of massive clumps and filaments of infrared dark clouds ${ }^{\star}, \star \star$
}

\author{
O. Miettinen
}

\author{
Department of Physics, University of Helsinki, PO Box 64, 00014 Helsinki, Finland \\ e-mail: oskari .miettinen@helsinki.fi
}

Received 3 September 2013 / Accepted 14 November 2013

\begin{abstract}
Context. Infrared dark clouds (IRDCs) provide a useful testbed in which to investigate the genuine initial conditions and early stages of massive-star formation.

Aims. We attempt to characterise the chemical properties of a sample of 35 massive clumps of IRDCs through multi-molecular line observations. We also search for possible evolutionary trends among the derived chemical parameters.

Methods. The clumps are studied using the MALT90 (Millimetre Astronomy Legacy Team $90 \mathrm{GHz}$ ) line survey data obtained with the Mopra $22 \mathrm{~m}$ telescope. The survey covers 16 different transitions near $90 \mathrm{GHz}$. The spectral-line data are used in concert with our previous LABOCA (Large APEX BOlometer CAmera) $870 \mu \mathrm{m}$ dust emission data.

Results. Eleven MALT90 transitions are detected towards the clumps at least at the $3 \sigma$ level. Most of the detected species $\left(\mathrm{SiO}, \mathrm{C}_{2} \mathrm{H}\right.$, $\mathrm{HNCO}, \mathrm{HCN}, \mathrm{HCO}^{+}, \mathrm{HNC}, \mathrm{HC}_{3} \mathrm{~N}$, and $\mathrm{N}_{2} \mathrm{H}^{+}$) show spatially extended emission towards many of the sources. Most of the fractional abundances of the molecules with respect to $\mathrm{H}_{2}$ are found to be comparable to those determined in other recent similar studies of IRDC clumps. We found that the abundances of $\mathrm{SiO}, \mathrm{HNCO}$, and $\mathrm{HCO}^{+}$are higher in IR-bright clumps than in IR-dark sources, reflecting a possible evolutionary trend. A hint of this trend is also seen for $\mathrm{HNC}$ and $\mathrm{HC}_{3} \mathrm{~N}$. An opposite trend is seen for the $\mathrm{C}_{2} \mathrm{H}$ and $\mathrm{N}_{2} \mathrm{H}^{+}$abundances. Moreover, a positive correlation is found between the abundances of $\mathrm{HCO}^{+}$and $\mathrm{HNC}$, and between those of $\mathrm{HNC}$ and $\mathrm{HCN}$. The HCN and HNC abundances also appear to increase as a function of the $\mathrm{N}_{2} \mathrm{H}^{+}$abundance. The $\mathrm{HNC} / \mathrm{HCN}$ and $\mathrm{N}_{2} \mathrm{H}^{+} / \mathrm{HNC}$ abundance ratios are derived to be near unity on average, while that of $\mathrm{HC}_{3} \mathrm{~N} / \mathrm{HCN}$ is $\sim 10 \%$. The $\mathrm{N}_{2} \mathrm{H}^{+} / \mathrm{HNC}$ ratio appears to increase as the clump evolves, while the $\mathrm{HNC} / \mathrm{HCO}^{+}$ratio shows the opposite behaviour.

Conclusions. The detected $\mathrm{SiO}$ emission is probably caused by shocks driven by outflows in most cases, although shocks resulting from the cloud formation process could also play a role. Shock-origin for the $\mathrm{HNCO}, \mathrm{HC}_{3} \mathrm{~N}$, and $\mathrm{CH}_{3} \mathrm{CN}$ emission is also plausible. The average $\mathrm{HNC} / \mathrm{HCN}$ ratio is in good agreement with those seen in other IRDCs, but gas temperature measurements would be neeeded to study its temperature dependence. Our results support the finding that $\mathrm{C}_{2} \mathrm{H}$ can trace the cold gas, and not just the photodissociation regions. The $\mathrm{HC}_{3} \mathrm{~N} / \mathrm{HCN}$ ratio appears to be comparable to the values seen in other types of objects, such as T Tauri disks and comets.
\end{abstract}

Key words. astrochemistry - stars: formation - ISM: abundances - ISM: clouds - ISM: molecules - radio lines: ISM

\section{Introduction}

The so-called infrared dark clouds (IRDCs) are, by definition, seen as dark absorption features against the Galactic mid-IR background radiation field (Pérault et al. 1996; Egan et al. 1998; Simon et al. 2006; Peretto \& Fuller 2009). Infrared dark clouds are a relatively new class of molecular clouds and, like molecular clouds in general, IRDCs represent the cradles of new stars. While the majority of IRDCs may serve as sites for the formation of low- to intermediate-mass stars and stellar clusters (Kauffmann \& Pillai 2010), observations have shown that some of them are capable of giving birth to high-mass $\left(M_{\star} \gtrsim 8 M_{\odot}\right.$; spectral type B3 or earlier) stars (e.g. Rathborne et al. 2006; Beuther \& Steinacker 2007; Chambers et al. 2009; Battersby et al. 2010; Zhang et al. 2011). Although IRDCs often show clear

* This publication is partly based on data acquired with the Atacama Pathfinder EXperiment (APEX) under programme 087.F-9315(A). APEX is a collaboration between the Max-Planck-Institut für Radioastronomie, the European Southern Observatory, and the Onsala Space Observatory.

$\star \star$ Appendices and Table 3 are available in electronic form at http://www . aanda.org signs of star-formation activity (such as point sources emitting IR radiation), some of the clumps and cores ${ }^{1}$ of IRDCs are found to be candidates for high-mass starless objects (e.g. Ragan et al. 2012; Tackenberg et al. 2012; Beuther et al. 2013; Sanhueza et al. 2013). These sources are ideal targets for examining the pristine initial conditions of high-mass star formation which are still rather poorly understood compared to the initial formation conditions of solar-type stars.

Besides the initial conditions, IRDCs provide us with the possibility of investigating the subsequent early stages of highmass star formation. These include the high-mass young stellar objects (YSOs), hot molecular cores (HMCs; e.g. Kurtz et al. 2000), and hyper- and ultracompact (UC) H II regions (e.g. Churchwell 2002; Hoare et al. 2007). From a chemical point of view, dense $\left(\gtrsim 10^{4} \mathrm{~cm}^{-3}\right)$ and cold $(\gtrsim 10 \mathrm{~K})$ starless IRDCs are expected to be characterised by the so-called dark-cloud chemistry, which is dominated by reactions between

\footnotetext{
Throughout the present paper, we use the term "clump" to refer to sources whose typical radii, masses, and mean densities are $\sim 0.2-1 \mathrm{pc}$, $\sim 10^{2}-10^{3} M_{\odot}$, and $\sim 10^{3}-10^{4} \mathrm{~cm}^{-3}$, respectively (cf. Bergin \& Tafalla 2007). The term "core" is used to describe a smaller (radius $\sim 0.1 \mathrm{pc}$ ) and denser object within a clump.
} 
electrically charged species (ions) and neutral species (e.g. Herbst \& Klemperer 1973; van Dishoeck \& Blake 1998). During this phase, the dust grains that are mixed with the gas are expected to accumulate icy mantles around them due to freezeout of some of the gas-phase species onto grain surfaces. If the source evolves to the HMC phase characterised by the dust temperature of $\gtrsim 100 \mathrm{~K}$, the ice mantles of dust grains are evaporated into the gas-phase leading to a rich and complex chemistry (e.g. Charnley 1995). Moreover, shocks occuring during the course of star formation due to outflows, for example, compress and heat the gas, and can fracture the grain mantles or even the grain cores leading to shock chemistry (e.g. Bachiller \& Perez Gutierrez 1997). As the chemistry of a star-forming region is very sensitive to prevailing physical conditions (temperature, density, ionisation degree), understanding the chemical composition is of great importance towards unveiling the physics of the early stages of high-mass star formation. Clearly, the chemical composition of the source changes with time, so the evolutionary timescale of the star-formation process can also be constrained by estimating the chemical age.

Establishing the chemical properties of certain types of interstellar clouds requires that large surveys be conducted. In past years, some multi-molecular line surveys of IRDC sources have already been published (Ragan et al. 2006; Beuther \& Sridharan 2007; Sakai et al. 2008, 2010; Gibson et al. 2009; Vasyunina et al. 2011 hereafter VLH11; Miettinen et al. 2011; Sanhueza et al. 2012 hereafter SJF12; Liu et al. 2013). However, most of the line survey studies of IRDCs performed so far are based on single-pointing observations in which case the spatial distribution of the studied species cannot be explored. To further characterise the chemical properties of IRDCs, the present paper presents a multi-line study of a sample of massive clumps within IRDCs selected from Miettinen (2012b; hereafter Paper I). As in Liu et al. (2013), the spectral-line data presented here were taken from the Millimetre Astronomy Legacy Team $90 \mathrm{GHz}$ (MALT90) survey (Foster et al. 2011; Jackson et al. 2013). As these data are based on mapping observations, we are able to study the spatial distribution of the line emission and the possible correlation between the emission of different species. This way we can examine the chemistry of several different species on clump scales and how the chemical properties vary among different sources or different evolutionary stages. After describing the source sample and data in Sect. 2, the observational results and analysis are presented in Sect. 3. In Sect. 4, we discuss the results, and summarise the paper in Sect. 5.

\section{Data}

\subsection{Source selection}

The source sample of the present paper was selected among the sources studied in Paper I where a sample of IRDC regions were investigated through mapping observations of the $870 \mu \mathrm{m}$ dust continuum emission with the APEX (Atacama Pathfinder EXperiment)/LABOCA (Large APEX BOlometer CAmera) bolometer array. From the four fields mapped with LABOCA, containing 91 clumps in total, altogether 35 clumps are included in the MALT90 survey $^{2}$. However, three of these clumps are only partly covered by MALT90 maps. The selected clumps are likely to encompass different evolutionary stages,

\footnotetext{
2 This overlap is not a coincidence in the sense that the MALT90 target sources were selected from the ATLASGAL (APEX Telescope Large Area Survey of the Galaxy) $870 \mu$ m survey (Schuller et al. 2009; Contreras et al. 2013).
}

ranging from IR-dark sources (13) to H II regions with bright IR emission (22 sources are associated with either IR point sources and/or extended-like IR emission). In Paper I, the clumps were classified into IR-dark and YSO-hosting ones depending on the Spitzer IRAC-colours of the point sources. Some of the studied clumps belong to filamentary IRDCs, most notably in the case of G11.36+0.80 (hereafter G11.36, etc.). Moreover, the sample includes clumps associated with the mid-IR bubble pair N10/11 (Churchwell et al. 2006), a potential site of ongoing triggered high-mass star formation (Watson et al. 2008).

In Fig. 1, we show the Spitzer $8 \mu \mathrm{m}$ images of our sources overlaid with contours showing the LABOCA $870 \mu \mathrm{m}$ dust emission. The angular sizes of the mid-IR images shown in Fig. 1 correspond to the MALT90 map sizes. The sources with their LABOCA peak positions are listed in Table 1. In this table, we also give the source kinematic distance $(d)$, effective radius $\left(R_{\text {eff }}\right)$, mass $(M), \mathrm{H}_{2}$ column density $\left[N\left(\mathrm{H}_{2}\right)\right]$, average $\mathrm{H}_{2}$ number density $\left[\left\langle n\left(\mathrm{H}_{2}\right)\right\rangle\right]$, and comments on the source appearance at IR wavelengths. The physical parameters shown in Table 1 were revised from those presented in Paper I, and are briefly described in Appendix A.

\subsection{MALT90 survey data}

The spectral-line data of the sources employed in the present study were observed as part of the MALT90 survey (PI: Jackson; see Foster et al. 2011, 2013; Jackson et al. 2013). The MALT90 observations cover the Galactic longitude ranges $3^{\circ}<l<20^{\circ}$ (1st quadrant) and $300^{\circ}<l<357^{\circ}$ (4th quadrant), and were targeting high-mass star-forming clumps in different stages of evolution. The survey was conducted with the $22 \mathrm{~m}$ Mopra telescope $^{3}$ in the on-the-fly (OTF) mapping mode during the austral winter in 2010-2012, covering the months of May to October. The OTF mapping was performed with the beam centre scanning in Galactic coordinates $(l, b)$ on a 3.4 $\times 3.4$ grid, where the beam FWHM (full width at half-maximum) is $38^{\prime \prime}$ at $90 \mathrm{GHz}$. The scanning speed was $3^{\prime \prime} 92 \mathrm{~s}^{-1}$. The step size between adjacent scanning rows was $12^{\prime \prime}$, i.e. $\sim 1 / 3$ of the beam FWHM, resulting in 17 rows per map. Each source was mapped twice by scanning in orthogonal directions ( $b$ versus $l$ ). One map took about half an hour to complete, and the total time spent on each field (two maps) was $1.18 \mathrm{~h}$. The mapping was carried out when the clump elevation was more than $35^{\circ}$ but less than $70^{\circ}$. The telescope pointing was checked every $1-1.5 \mathrm{~h}$ on $\mathrm{SiO}$ maser sources, and was found to be better than $\sim 10^{\prime \prime}$.

The spectrometer used was the MOPra Spectrometer (MOPS) which is a digital filter bank ${ }^{4}$. The MOPS spectrometer was tuned to a central frequency of $89.690 \mathrm{GHz}$, and the $8 \mathrm{GHz}$ wide frequency band of MOPS was split into 16 subbands of 137.5 MHz each (4 096 channels), resulting in a velocity resolution of $\sim 0.11 \mathrm{~km} \mathrm{~s}^{-1}$ in each band (the so-called zoom mode). The typical system temperatures during the observations were in the range $T_{\text {sys }} \sim 180-300 \mathrm{~K}$, and the typical rms noise level was $\sim 250 \mathrm{mK}$ per $0.11 \mathrm{~km} \mathrm{~s}^{-1}$ channel. The output intensity scale given by the Mopra/MOPS system was $T_{\mathrm{A}}^{\star}$, i.e. the antenna temperature corrected for the atmospheric attenuation. The observed intensities were converted to the main-beam brightness

\footnotetext{
3 The Mopra radio telescope is part of the Australia Telescope National Facility which is funded by the Commonwealth of Australia for operation as a National Facility managed by CSIRO.

4 The University of New South Wales Digital Filter Bank used for the observations with the Mopra Telescope was provided with support from the Australian Research Council.
} 
O. Miettinen: A MALT90 chemical study of clumps within IRDCs
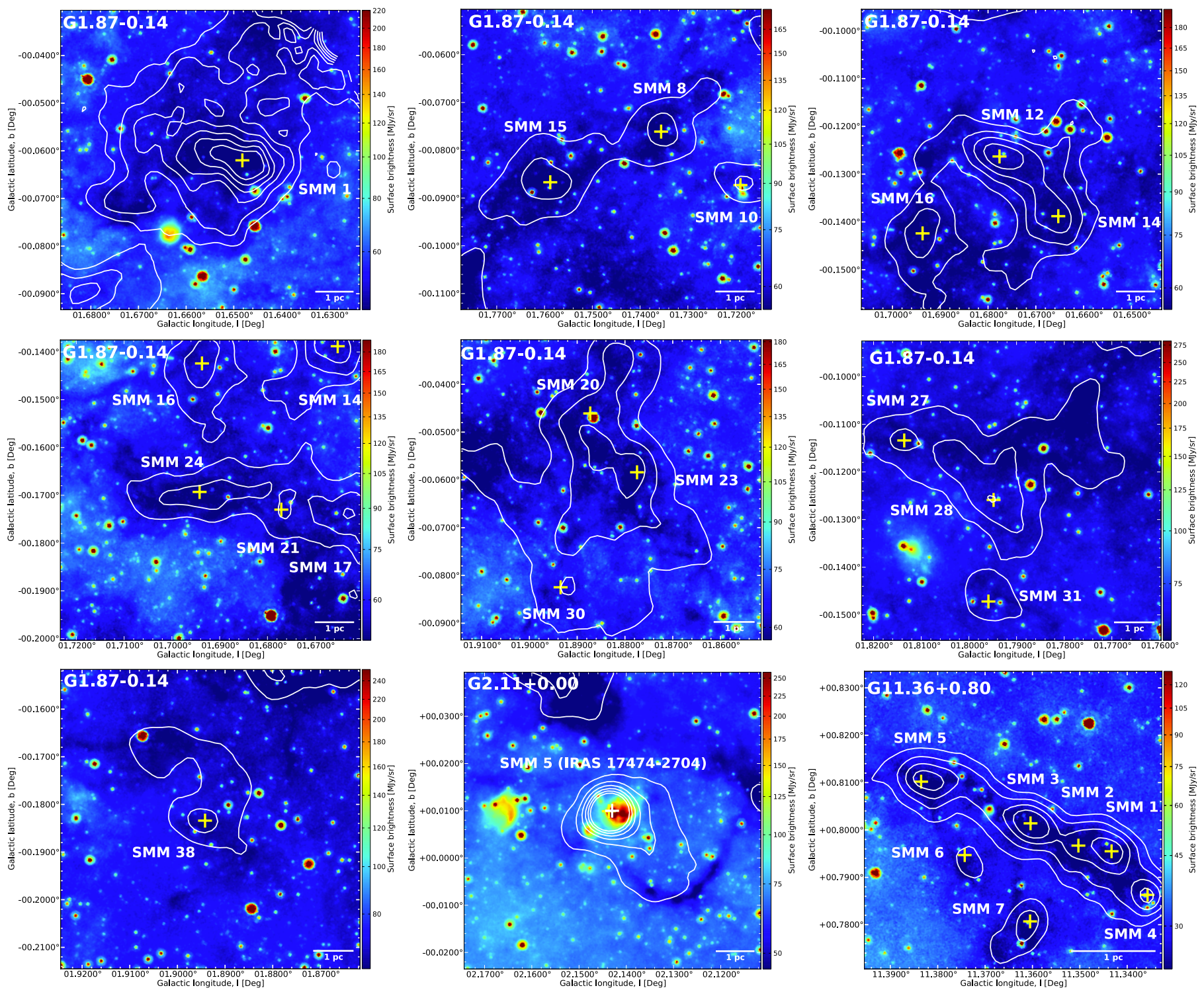

Fig. 1. Spitzer/IRAC $8 \mu \mathrm{m}$ images of the clumps and filaments studied in the present paper. The images are shown with logarithmic scaling, and the colour bars indicate the surface-brightness scale in $\mathrm{MJy} \mathrm{sr}^{-1}$. The images are overlaid with contours of LABOCA $870 \mu$ m dust continuum emission as in Paper I (starting from $3 \sigma$ and going in steps of $3 \sigma$, where $3 \sigma$ is $0.27,0.18,0.12$, and $0.14 \mathrm{Jy}_{\text {beam }}^{-1}$ for fields G1.87, G2.11, G11.36, and G13.22, respectively). The $870 \mu \mathrm{m}$ peak positions of the clumps are denoted by yellow plus signs. In each panel, a scale bar indicating the 1 pc projected length is shown, with the assumption of line-of-sight distance given in Col. (4) of Table 1. The source nomenclature follows that in Paper I. The clumps G1.87-SMM 10, 17, and G13.22-SMM 10 are only partly covered by the MALT90 maps. The white plus sign towards G2.11-SMM 5 shows the position of the UC Hil region from Becker et al. (1994; as seen at $5 \mathrm{GHz}$ ) and the $18 \mathrm{~cm} \mathrm{OH}$ maser from Argon et al. (2000); the two positions overlap, and are very close to the $870 \mu \mathrm{m}$ peak position.

temperature scale by $T_{\mathrm{MB}}=T_{\mathrm{A}}^{\star} / \eta_{\mathrm{MB}}$, where $\eta_{\mathrm{MB}}$ is the mainbeam efficiency. The value of $\eta_{\mathrm{MB}}$ is 0.49 at $86 \mathrm{GHz}$ and 0.44 at $110 \mathrm{GHz}$ (Ladd et al. 2005). Extrapolation using the Ruze formula gives the $\eta_{\mathrm{MB}}$ values in the range $0.49-0.46$ for the 86.75-93.17 GHz frequency range of MALT90.

The 16 spectral-line transitions mapped simultaneously in the MALT90 survey are listed in Table 2 . In this table, we give some spectroscopic parameters of the spectral lines, and in the last column we also provide comments on each transition and information provided by the lines. The MALT90 datafiles are publicly available and can be downloaded through the Australia Telescope Online Archive (ATOA) ${ }^{5}$.

http://atoa.atnf.csiro.au/MALT90

\section{Results and analysis}

\subsection{Spatial distributions of the spectral-line emission}

In this subsection, we present the integrated intensity maps of the spectral lines detected towards the clumps. Besides the maps of integrated intensity, or the 0th moment maps, the MALT90 data archive contains the uncertainty maps of the 0th moment images. The typical $1 \sigma$ error, in units of integrated $T_{\mathrm{MB}}$, was found to be $\sim 0.6-0.7 \mathrm{~K} \mathrm{~km} \mathrm{~s}^{-1}$. The $\operatorname{HNCO}\left(4_{1,3}-3_{1,2}\right)$ maps were an exception, however, as in many cases they were found to be very noisy with $1 \sigma$ values of $\sim 3-8 \mathrm{~K} \mathrm{~km} \mathrm{~s}^{-1}$. Moreover, the $\mathrm{HCN}$ and $\mathrm{HCO}^{+}$maps towards G1.87-SMM 38 were corrupted and could not be used.

The 0th moment maps are presented in Figs. B.1-B.14, where the white contours showing the spectral-line emission are 

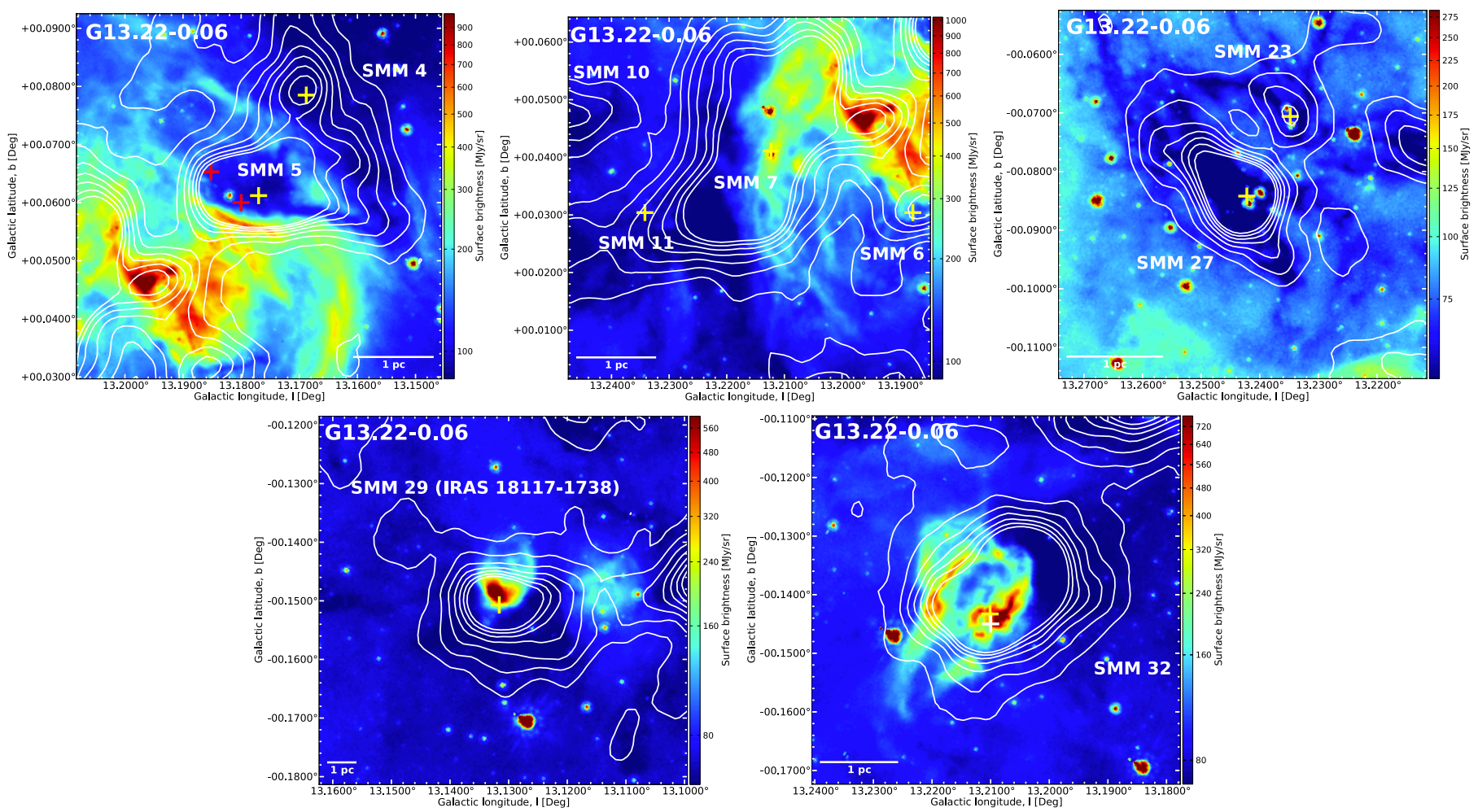

Fig. 1. continued. The red plus signs near G13.22-SMM 5 indicate the positions of the $6.7 \mathrm{GHz}$ Class II methanol masers from Szymczak et al. (2000; upper; see also Deharveng et al. 2010) and Pandian et al. (2008; lower). In the G13.22-SMM 32 panel, the white plus sign marks the position of the compact $\mathrm{H}$ II region from Wink et al. (1982), as observed at $4.9 \mathrm{GHz}$.

overlaid on the Spitzer $8 \mu \mathrm{m}$ images. The red plus signs mark the LABOCA peak positions to guide the eye. The line emission was deemed to be real if the integrated intensity was detected at least at the $3 \sigma$ level. Only maps of detected line emission are presented. In some cases, the contours are plotted to start at a stronger emission level than $3 \sigma$ for illustrative purposes.

As can be seen from Figs. B.4 and B.11 (cf. Fig. 1), the LABOCA $870 \mu \mathrm{m}$ emission peaks of G1.87-SMM 17 and G13.22-SMM 10 are not covered by the MALT90 maps. For most of the lines the detection rate is generally high. In particular, $\mathrm{HNC}(1-0)$ and $\mathrm{N}_{2} \mathrm{H}^{+}(1-0)$ were detected towards all fields. In addition, $\mathrm{SiO}(2-1), \mathrm{C}_{2} \mathrm{H}\left(1_{3 / 2,2}-0_{1 / 2,1}\right), \mathrm{HCN}(1-0)$, and $\mathrm{HCO}^{+}(1-0)$ were seen towards $86-93 \%$ of the fields. As mentioned above, the $\operatorname{HNCO}\left(4_{1,3}-3_{1,2}\right)$ maps were often very noisy, and emission was not detected even in the cases where the noise level was at the normal level of $0.6-0.7 \mathrm{~K} \mathrm{~km} \mathrm{~s}^{-1}$. The $\mathrm{HC}^{13} \mathrm{CCN}(10-9), \mathrm{H} 41 \alpha$, and ${ }^{13} \mathrm{C}^{34} \mathrm{~S}(2-1)$ lines were not detected in any of the sources. Moreover, only two fields show weak traces of ${ }^{13} \mathrm{C}^{32} \mathrm{~S}(2-1)$ emission, which explains the nondetections of the rarer isotopologue ${ }^{13} \mathrm{C}^{34} \mathrm{~S}$.

\subsection{Spectra and line parameters}

The beam-averaged ( $\left.38^{\prime \prime}\right)$ spectra were extracted from the data cubes towards the LABOCA peak positions of the clumps and towards selected line emission peaks. The spectra were analysed using the CLASS90 programme of the GILDAS software package ${ }^{6}$. Linear (first-order) to third-order baselines were determined from velocity ranges without line-emission features,

\footnotetext{
6 Grenoble Image and Line Data Analysis Software is provided and actively developed by IRAM, and is available at http://www.iram. fr/IRAMFR/GILDAS
}

and then subtracted from the spectra. The resulting $1 \sigma$ rms noise levels were in the range $\sim 0.2-0.4 \mathrm{~K}$ on the $T_{\mathrm{MB}}$ scale. The spectra are presented in Figs. C.1-C.33. The spectra are overlaid with single Gaussian/hf-structure fits (see below). The coordinates of the line emission peaks in the clump regions are shown in the top left corners of the corresponding panels (e.g. the $\mathrm{HCO}^{+}$and $\mathrm{HNC}$ spectra in Fig. C.1). When only a weak trace of emission was seen in the 0th moment map, there was no detectable line in the extracted spectrum. This was particularly the case for the ${ }^{13} \mathrm{CS}(2-1)$ transition.

As described in Table 2, of the detected lines only $\mathrm{SiO}(2-1)$ and $\mathrm{HCO}^{+}(1-0)$ have no hf structure. The $\mathrm{SiO}$ and $\mathrm{HCO}^{+}$ lines were therefore fitted with a single Gaussian profile using CLASS90. For the rest of the detected lines, we used the hfs method of CLASS90 to fit the hf structure, although the hf components were not fully resolved in any of the sources because of large linewidths typical of massive clumps. The relative positions (in frequency or velocity) and relative strengths of the hf components were searched from the literature (see the references in Table 2) or via the Splatalogue spectral line database ${ }^{7}$. The derived spectral-line parameters are listed in Table 3 at the end of the paper. In Cols. 3 and 4 we give the LSR velocity of the emission $\left(v_{\mathrm{LSR}}\right)$ and FWHM linewidth $(\Delta v)$, respectively. Columns 5 and 6 list the peak intensities $\left(T_{\mathrm{MB}}\right)$ and integrated line intensities $\left(\int T_{\mathrm{MB}} \mathrm{d} v\right)$. The quoted uncertainties in these parameters represent the formal fitting errors (i.e. calibration uncertainties are not taken into account). The values of $T_{\mathrm{MB}}$ and $\int T_{\mathrm{MB}} \mathrm{d} v$ were either determined for a blended group of hf components or, when resolved, for the strongest line which itself could be a multiplet of individual hf lines.

\footnotetext{
http://splatalogue.net/
} 
O. Miettinen: A MALT90 chemical study of clumps within IRDCs

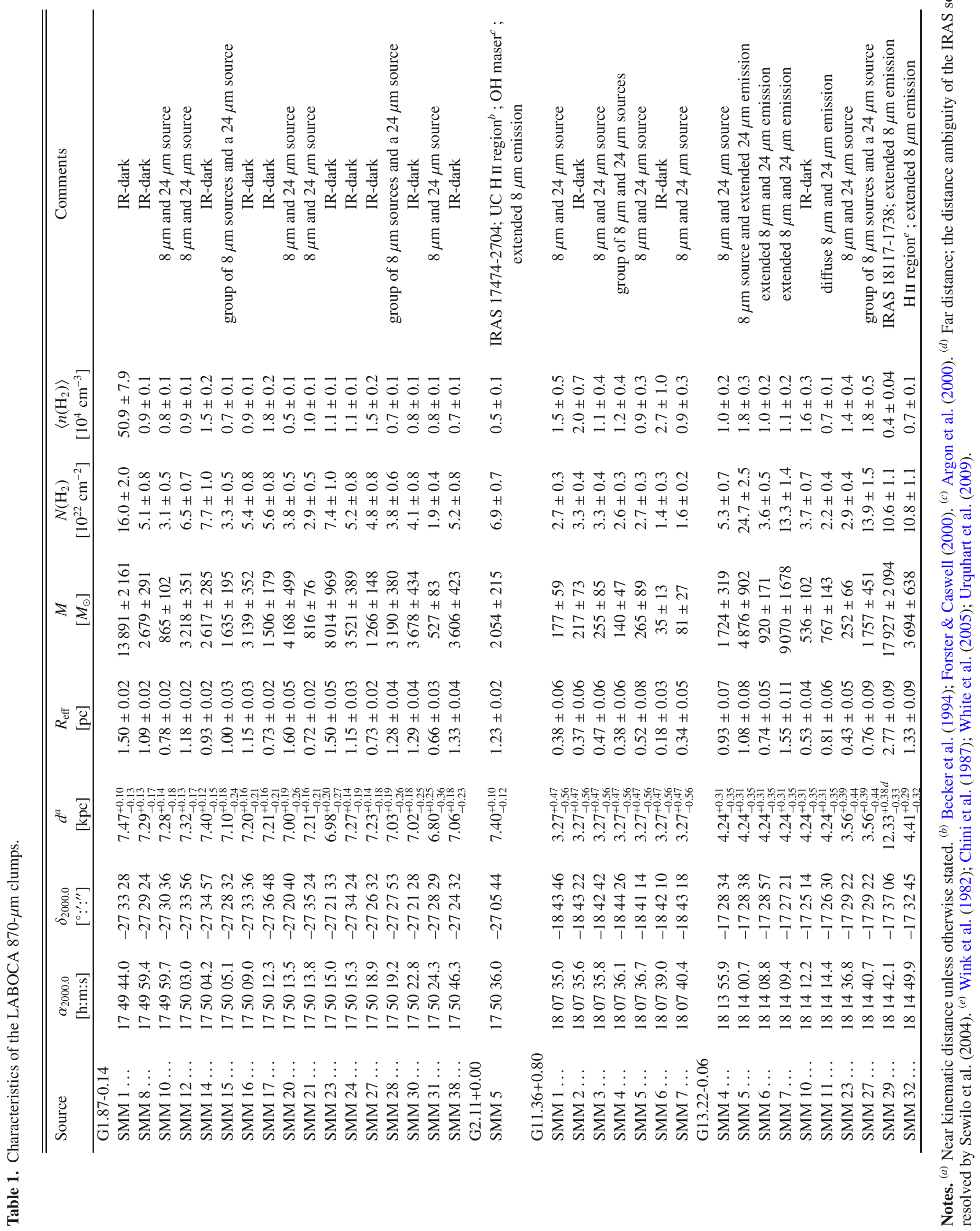


Table 2. MALT90 spectral-line transitions.

\begin{tabular}{|c|c|c|c|c|}
\hline Transition $^{a}$ & $\begin{array}{c}v^{b} \\
{[\mathrm{MHz}]}\end{array}$ & $\begin{array}{c}E_{\mathrm{u}} / k_{\mathrm{B}}^{c} \\
{[\mathrm{~K}]}\end{array}$ & $\begin{array}{c}n_{\text {crit }}{ }^{d} \\
{\left[\mathrm{~cm}^{-3}\right]}\end{array}$ & Comments $^{e}$ \\
\hline $\mathrm{H}^{13} \mathrm{CO}^{+}(J=1-0)$ & 86754.330 & 4.16 & $1.6 \times 10^{5}$ & $\begin{array}{l}\text { high-density and ionisation tracer; } \\
J=1-0 \text { is split into six hyperfine (hf) components }{ }^{f}\end{array}$ \\
\hline $\mathrm{SiO}(J=2-1)$ & 86847.010 & 6.25 & $2 \times 10^{6}$ & shocked-gas/outflow tracer \\
\hline $\mathrm{HN}^{13} \mathrm{C}(J=1-0)$ & 87090.859 & 4.18 & $2 \times 10^{5 g}$ & $\begin{array}{l}\text { high-density tracer; } \\
J=1-0 \text { is split into } 11 \mathrm{hf} \text { components } \\
\text { with four having a different frequency }\end{array}$ \\
\hline $\mathrm{C}_{2} \mathrm{H}\left(N_{J, F}=1_{3 / 2,2}-0_{1 / 2,1}\right)$ & 87316.925 & 4.19 & $2 \times 10^{5 i}$ & $\begin{array}{l}\text { a tracer of photodissociation regions (PDRs); } \\
N_{J}=1_{3 / 2}-0_{1 / 2} \text { is split into three hf components }{ }^{j}\end{array}$ \\
\hline $\operatorname{HNCO}\left(J_{K_{a}, K_{b}}=4_{0,4}-3_{0,3}\right)$ & 87925.238 & 10.55 & $4.5 \times 10^{6 k}$ & $\begin{array}{l}\text { hot core and shock-chemistry tracer; } \\
\text { six hf components } ; a \text {-type transition }\left(\Delta K_{a}=0\right)\end{array}$ \\
\hline $\operatorname{HNCO}\left(J_{K_{a}, K_{b}}=4_{1,3}-3_{1,2}\right)$ & 88239.027 & 53.86 & $2.3 \times 10^{6 k}$ & six hf components ${ }^{l} ; a$-type transition $\left(\Delta K_{a}=0\right)$ \\
\hline $\operatorname{HCN}(J=1-0)$ & 88631.847 & 4.25 & $2.4 \times 10^{6}$ & $\begin{array}{l}\text { high-density and infall tracer; } \\
J=1-0 \text { is split into three hf components }{ }^{m}\end{array}$ \\
\hline $\mathrm{HCO}^{+}(J=1-0)$ & 89188.526 & 4.28 & $1.7 \times 10^{5}$ & $\begin{array}{l}\text { high-density, infall, and ionisation tracer; } \\
\text { enhanced in outflows due to shock-induced UV radiation }{ }^{n}\end{array}$ \\
\hline $\mathrm{HC}^{13} \mathrm{CCN}(J=10-9, F=9-8)$ & 90593.059 & 23.91 & $1.7 \times 10^{5 o}$ & hot-core tracer; five hf components \\
\hline $\mathrm{HNC}(J=1-0)$ & 90663.572 & 4.35 & $2.9 \times 10^{5}$ & high-density tracer; three hf components \\
\hline${ }^{13} \mathrm{C}^{34} \mathrm{~S}(J=2-1)$ & 90926.036 & 6.54 & $4.3 \times 10^{5 p}$ & high-density tracer \\
\hline $\mathrm{HC}_{3} \mathrm{~N}(J=10-9)$ & 91199.796 & 24.01 & $5.3 \times 10^{5}$ & $\begin{array}{l}\text { high-density/hot-core tracer; } \\
\text { six hf components }\end{array}$ \\
\hline $\mathrm{CH}_{3} \mathrm{CN}\left(J_{K}=5_{1}-4_{1}\right)$ & 91985.316 & 20.39 & $4 \times 10^{5 q}$ & hot-core tracer; seven hf components \\
\hline $\mathrm{H} 41 \alpha$ & 92034.475 & $89.5^{r}$ & $\sim 5^{s}$ & $\begin{array}{l}\text { ionised gas tracer; the principal quantum number } \\
\text { changes from } n=42 \text { to } 41 \rightarrow \alpha \text {-type radio recombination line }\end{array}$ \\
\hline${ }^{13} \mathrm{CS}(J=2-1)$ & 92494.303 & 6.66 & $5 \times 10^{5 k}$ & high-density tracer; three hf components \\
\hline $\mathrm{N}_{2} \mathrm{H}^{+}(J=1-0)$ & 93173.480 & 4.47 & $1.5 \times 10^{5}$ & $\begin{array}{l}\text { high-density/CO-depleted gas tracer; } \\
J=1-0 \text { line has } 15 \text { hf components out of which } \\
\text { seven have a different frequency }\end{array}$ \\
\hline
\end{tabular}

Notes. ${ }^{(a)}$ The rotational transitions here occur in the vibrational ground state $(v=0) .{ }^{(b)}$ Rest frequencies adopted from the MALT90 webpage (http://malt90.bu.edu/parameters.html). ${ }^{(c)}$ Upper-state energy divided by the Boltzmann constant. ${ }^{(d)}$ Critical density at $15 \mathrm{~K}$ unless otherwise stated. Unless otherwise stated, the Einstein $A$ coefficients and collision rates were adopted from the Leiden Atomic and Molecular Database (LAMDA (Schöier et al. 2005); http://home.strw.leidenuniv.nl/ moldata/). (e) Comments on the species and transition in question. ${ }^{(f)}$ See e.g. Schmid-Burgk et al. (2004). ${ }^{(g)}$ Collision rate for HNC from LAMDA was used to estimate $n_{\text {crit. }}{ }^{\left({ }^{(h)}\right.}$ van der Tak et al. $(2009)$; Padovani et al. (2011). ${ }^{(i)}$ From Lo et al. (2009). ${ }^{(j)}$ Reitblat (1980); Padovani et al. (2009); Spielfiedel et al. $(2012) .{ }^{(k)} n_{\text {crit }}$ at $20 \mathrm{~K} .{ }^{(l)}$ Lapinov et al. (2007). ${ }^{(m)}$ See e.g. Cao et al. (1993). ${ }^{(n)}$ Rawlings et al. (2000), 2004. ${ }^{(o)} n_{\text {crit }}$ was estimated using the Einstein $A$ coefficient from the Cologne Database for Molecular Spectroscopy (CDMS; Müller et al. 2005; http://www.astro.uni-koeln.de/cdms) and the $\mathrm{HC}_{3} \mathrm{~N}$ collision rate at $15 \mathrm{~K}$ from LAMDA. ${ }^{(p)} n_{\text {crit }}$ was estimated using the Einstein $A$ coefficient from CDMS and the ${ }^{12} \mathrm{C}^{34} \mathrm{~S}$ collision rate at $20 \mathrm{~K}$ from LAMDA. ${ }^{(q)}$ From SJF12. ${ }^{(r)}$ The energy of the $n=42$ level $\left(E=-E_{0} / n^{2}\right.$, where $\left.E_{0}=13.6 \mathrm{eV}\right)$. ${ }^{(s)}$ Critical electron density at $10^{4} \mathrm{~K}$ (see Appendix D.1 in Gordon \& Sorochenko 2009). ${ }^{(t)}$ See Table 2 in Pagani et al. (2009) and Table 1 in Keto \& Rybicki (2010).

Some of the $\mathrm{HCN}, \mathrm{HCO}^{+}, \mathrm{HNC}$, and $\mathrm{N}_{2} \mathrm{H}^{+}$lines were found to show double-peaked profiles caused by gas kinematics, not by hf splitting. The blue-skewed profiles, i.e. those with blueshifted peaks stronger than red peaks (e.g. the HCN spectrum towards G1.87-SMM 31; Fig. C.15) could be the manifestation of large-scale collapse motions (e.g. Zhou et al. 1993; Myers et al. 1996; Lee et al. 1999; Gao et al. 2009). In contrast, the redskewed profiles with stronger red peaks and weaker blue peaks suggest that the envelope is expanding (e.g. Thompson \& White 2004; Velusamy et al. 2008; Gao \& Lou 2010); see, for example, the HCN and HNC lines towards G1.87-SMM 1 (Fig. C.1). In Table 2, for double-peaked lines we also give the line parameters of both the blue and red peaks separately derived through fitting a single Gaussian to each peak. Finally, in a few cases (e.g. the $\mathrm{HCO}^{+}$line towards G1.87-SMM 1 and G2.11-SMM5) we observe more than one velocity component along the line of sight. These additional velocity components are typically much weaker than the main component and do not significantly contribute to the integrated intensity maps that were constructed by integrating over the whole velocity range.

\subsection{Line optical thicknesses and excitation temperatures}

The optical thickness of the line emission $(\tau)$ and the excitation temperature $\left(T_{\text {ex }}\right)$ could not be derived through fitting the hf structure in all cases. The main reasons for this are the blending of the hf components and limited signal-to-noise $(\mathrm{S} / \mathrm{N})$ ratio of the spectra. The average of the $T_{\text {ex }}$ values that could be directly derived via the $\mathrm{hfs}$ method was adopted for the rest of the given lines. In a few cases we were able to derive the optical thickness by comparing the intensities of two different isotopologues of the same species, namely $\mathrm{HCO}^{+} / \mathrm{H}^{13} \mathrm{CO}^{+}$and $\mathrm{HNC} / \mathrm{HN}^{13} \mathrm{C}$ (cf. SJF12; their Eq. (6)). For this analysis, we adopted the Galactocentric distance-dependent $\left[{ }^{12} \mathrm{C}\right] /\left[{ }^{13} \mathrm{C}\right]$ ratio from Wilson \& Rood (1994):

$\frac{\left[{ }^{12} \mathrm{C}\right]}{\left[{ }^{13} \mathrm{C}\right]}=7.5 \times R_{\mathrm{GC}}[\mathrm{kpc}]+7.6$.

The optical thickness ratio between the two isotopologues was assumed to be equal to that given by Eq. (1). Using the derived value of $\tau$, the value of $T_{\mathrm{ex}}$ was calculated using the familiar antenna equation (see e.g. Eq. (A.1) of Miettinen 2012a). 
When $T_{\text {ex }}$ could not be derived/assumed as described above, we assumed that it is equal to $E_{\mathrm{u}} / k_{\mathrm{B}}$ in the case of linear molecules ( $\mathrm{SiO}$ and $\mathrm{HC}_{3} \mathrm{~N}$ in our case), and that $T_{\mathrm{ex}}=2 / 3 \times$ $E_{\mathrm{u}} / k_{\mathrm{B}}$ in the case of $\mathrm{HNCO}$, which is a nearly prolate asymmetric top molecule, and $\mathrm{CH}_{3} \mathrm{CN}$, which is a prolate symmetric top. These $T_{\text {ex }}$ values, used to estimate the line optical thickness, lead to the lower limit to the molecular column density (e.g. Hatchell et al. 1998; see also Miettinen 2012a). The values of $\tau$ and $T_{\text {ex }}$ are listed in Cols. 7 and 8 in Table 3. In case the line has a hf structure, the $\tau$ value refers to the sum of the peak optical thicknesses of individual hf components. For lines with blended hf multiplets, this total optical thickness was derived by dividing the optical thickness of the strongest hf component by its statistical weight.

\subsection{Column densities and fractional abundances}

The beam-averaged column densities of the molecules, $N(\mathrm{~mol})$, were calculated by using the standard local thermodynamic equilibrium (LTE) formulation

$N(\mathrm{~mol})=\frac{3 h \epsilon_{0}}{2 \pi^{2}} \frac{1}{\mu^{2} S} \frac{Z_{\mathrm{rot}}\left(T_{\mathrm{ex}}\right)}{g_{K} g_{I}} \mathrm{e}^{E_{\mathrm{u}} / k_{\mathrm{B}} T_{\mathrm{ex}}} F\left(T_{\mathrm{ex}}\right) \int \tau(v) \mathrm{d} v$,

where $h$ is the Planck constant, $\epsilon_{0}$ is the vacuum permittivity, $\mu$ is the permanent electric dipole moment, $S$ is the line strength, $Z_{\text {rot }}$ is the rotational partition function, $g_{K}$ is the $K$-level degeneracy, $g_{I}$ is the reduced nuclear spin degeneracy (see e.g. Turner 1991), and $F\left(T_{\mathrm{ex}}\right) \equiv\left(\mathrm{e}^{h v / k_{\mathrm{B}} T_{\mathrm{ex}}}-1\right)^{-1}$. Here, the electric dipole moment matrix element is defined as $\left|\mu_{\mathrm{ul}}\right| \equiv \mu^{2} S / g_{\mathrm{u}}$, where $g_{\mathrm{u}}=2 J+1$ is the rotational degeneracy of the upper state (Townes \& Schawlow 1975). The values of the product $\mu^{2} S$ were taken from the Splatalogue database. For linear molecules, $g_{K}=g_{I}=1$ for all levels (Turner 1991). As an asymmetric top, HNCO has $g_{K}=1$ (no $K$-level degeneracy), and because of absence of identical interchangeable nuclei, $g_{I}$ is also equal to unity. For the detected $\mathrm{CH}_{3} \mathrm{CN}$ line, $g_{K}=2$ because $K \neq 0$ (degeneration among the $K$-type doublets), and $g_{I}=1 / 4$ because $K \neq 3 n$, where $n$ is an integer (Turner 1991).

The partition function of the linear molecules was approximated as

$Z_{\mathrm{rot}}\left(T_{\mathrm{ex}}\right) \simeq \frac{k_{\mathrm{B}} T_{\mathrm{ex}}}{h B}+\frac{1}{3}$

where $B$ is the rotational constant. The above expression is appropriate for heteropolar molecules at the high temperature limit of $h B / k_{\mathrm{B}} T_{\mathrm{ex}} \ll 1$. For HNCO, the partition function was calculated as

$Z_{\mathrm{rot}}\left(T_{\mathrm{ex}}\right)=\sqrt{\frac{\pi\left(k_{\mathrm{B}} T_{\mathrm{ex}}\right)^{3}}{h^{3} A B C}}$,

where $A, B$, and $C$ are the three rotational constants. For $\mathrm{CH}_{3} \mathrm{CN}$, the partition function is given by Eq. (4) multiplied by $1 / 3$ because of the three interchangeable H-nuclei (Turner 1991). We note that for the prolate symmetric top molecule $\mathrm{CH}_{3} \mathrm{CN}, B=C$ in Eq. (4).

When the line profile has a Gaussian shape, the last integral term in Eq. (2) can be expressed as a function of the FWHM linewidth and peak optical thickness of the line as

$$
\int \tau(v) \mathrm{d} v=\frac{\sqrt{\pi}}{2 \sqrt{\ln 2}} \Delta v \tau \simeq 1.064 \Delta v \tau .
$$

Moreover, if the line emission is optically thin $(\tau \ll 1), T_{\mathrm{MB}} \propto \tau$, and $N(\mathrm{~mol})$ can be computed from the integrated line intensity (see e.g. Eq. (A.4) of Miettinen (2012a)). The values of $\tau$ listed in Table 2 were used to decide by which method (from the linewidth or integrated intensity) the column density was computed. Our analysis assumed that the line emission fills the telescope beam, i.e. that the beam filling factor is unity. As can be seen in the 0th moment maps, the line emission is often extended with respect to the $38^{\prime \prime}\left(0^{\circ} .011\right)$ beam size. However, this does not necessarily mean that the assumption of unity filling factor is correct. If the gas is clumpy within the beam area, the true filling factor is $<1$. In this case, the derived beam-averaged column density is only a lower limit to the source-averaged value.

The fractional abundances of the molecules were calculated by dividing the molecular column density by the $\mathrm{H}_{2}$ column density, $x(\mathrm{~mol})=N(\mathrm{~mol}) / N\left(\mathrm{H}_{2}\right)$. To be directly comparable to the line observations, the $N\left(\mathrm{H}_{2}\right)$ values were derived from the LABOCA dust continuum maps smoothed to the MALT90 resolution of $38^{\prime \prime}$.

The beam-averaged column densities and abundances with respect to $\mathrm{H}_{2}$ are given in the last two columns of Table 3. Statistics of these parameters are given in Table 4, where we provide the mean, median, standard deviation (std), and minimum and maximum values of the sample (the values for additional velocity components have been neglected). This table provides an easier way to compare the derived molecular column densities and abundances with those found in other studies.

\subsection{Abundance ratios and correlations}

As the purpose of the present study is to examine the chemistry of the sources, we computed the abundance ratios between selected molecules. In Table 5, we list the $\mathrm{HNC} / \mathrm{HCN}$, $\mathrm{HNC} / \mathrm{HCO}^{+}, \mathrm{N}_{2} \mathrm{H}^{+} / \mathrm{HCO}^{+}, \mathrm{N}_{2} \mathrm{H}^{+} / \mathrm{HNC}$, and $\mathrm{HC}_{3} \mathrm{~N} / \mathrm{HCN}$ column density ratios for the clumps. The quoted uncertainties were propagated from those of the column densities.

We also searched for possible correlations between different parameter pairs. As shown in the top left panel of Fig. 2, there is a hint that the fractional abundance of HCN decreases as a function of the $\mathrm{H}_{2}$ column density. A least squares fit to the data points yields $\log [x(\mathrm{HCN})]=(24.64 \pm 13.53)-(1.46 \pm$ $0.60) \log \left[N\left(\mathrm{H}_{2}\right)\right]$, with the linear Pearson correlation coefficient of $r=-0.55$. For this plot, the $\mathrm{H}_{2}$ column densities were derived from the LABOCA maps smoothed to the resolution of the MALT90 data. In the rest of the panels in Fig. 2, we show the correlations found between different molecular fractional abundances. The top right panel plots the HCN abundance as a function of $x(\mathrm{HNC})$. The overplotted linear regression model is of the form $\log [x(\mathrm{HCN})]=(-2.36 \pm 3.46)+$ $(0.70 \pm 0.39) \log [x(\mathrm{HNC})]$, with the Pearson's $r$ of 0.45 . The middle left panel plots the HNC abundance as a function of the $\mathrm{HCO}^{+}$abundance. A positive corrrelation is found, and the fitted linear relationship is of the form $\log [x(\mathrm{HNC})]=(0.42 \pm$ $1.32)+(0.99 \pm 0.14) \log \left[x\left(\mathrm{HCO}^{+}\right)\right](r=0.80)$. The middle right panel shows the HCN abundance plotted as a function of $x\left(\mathrm{~N}_{2} \mathrm{H}^{+}\right)$. Again, the data suggest a positive correlation, and the functional form of the linear fit is $\log [x(\mathrm{HCN})]=(3.56 \pm 3.38)+$ $(1.34 \pm 0.37) \log \left[x\left(\mathrm{~N}_{2} \mathrm{H}^{+}\right)\right](r=0.71)$. The bottom panel shows the $\mathrm{HNC}$ abundance as a function of the $\mathrm{N}_{2} \mathrm{H}^{+}$abundance. Here, the correlation coefficient is only 0.39 , and no linear fit is shown.

\section{Discussion}

In this section, we discuss the obtained results for each individual species separately. We compare our results with those 
Table 4. Statistics of column densities and fractional abundances.

\begin{tabular}{|c|c|c|c|c|c|}
\hline \multicolumn{6}{|c|}{ All clumps } \\
\hline Quantity & Mean & Median & $\mathrm{Std}^{a}$ & Min. & Max. \\
\hline$N\left(\mathrm{H}^{13} \mathrm{CO}^{+}\right)^{b}$ & $7.4(12)$ & $6.4(12)$ & $2.3(12)$ & $5.8(12)$ & $1.0(13)$ \\
\hline$x\left(\mathrm{H}^{13} \mathrm{CO}^{+}\right)^{b}$ & $9.7(-11)$ & $1.1(-10)$ & $5.1(-11)$ & $4.0(-11)$ & $1.4(-10)$ \\
\hline$N(\mathrm{SiO})$ & $1.9(13)$ & $1.5(13)$ & $1.3(13)$ & $5.5(12)$ & $4.8(13)$ \\
\hline$x(\mathrm{SiO})$ & $5.7(-10)$ & $3.6(-10)$ & $5.1(-10)$ & $4.0(-11)$ & $1.8(-9)$ \\
\hline$N\left(\mathrm{HN}^{13} \mathrm{C}\right)$ & 1.1(13) & 1.1(13) & $2.5(12)$ & $9.6(12)$ & $1.5(13)$ \\
\hline$x\left(\mathrm{HN}^{13} \mathrm{C}\right)$ & $1.2(-10)$ & $1.2(-10)$ & $1.6(-11)$ & $1.0(-10)$ & $1.4(-10)$ \\
\hline$N\left(\mathrm{C}_{2} \mathrm{H}\right)$ & $4.3(14)$ & $3.4(14)$ & $3.3(14)$ & $7.8(13)$ & $1.2(15)$ \\
\hline$x\left(\mathrm{C}_{2} \mathrm{H}\right)$ & $8.1(-9)$ & $6.3(-9)$ & $6.1(-9)$ & $1.8(-9)$ & $2.4(-8)$ \\
\hline$N(\mathrm{HNCO})$ & $2.2(14)$ & $1.4(14)$ & $2.2(14)$ & $5.4(12)$ & $7.9(14)$ \\
\hline$x(\mathrm{HNCO})$ & $6.6(-9)$ & $5.0(-9)$ & $5.8(-9)$ & $1.3(-10)$ & $1.9(-8)$ \\
\hline$N(\mathrm{HCN})$ & $5.9(14)$ & $8.7(13)$ & $1.4(15)$ & $1.6(13)$ & $5.5(15)$ \\
\hline$x(\mathrm{HCN})$ & $2.1(-8)$ & $2.0(-9)$ & $5.2(-8)$ & $2.7(-10)$ & $2.1(-7)$ \\
\hline$N\left(\mathrm{HCO}^{+}\right)$ & $3.0(13)$ & $9.7(12)$ & $5.6(13)$ & $1.1(12)$ & $2.6(14)$ \\
\hline$x\left(\mathrm{HCO}^{+}\right)$ & $5.6(-10)$ & $3.3(-10)$ & $6.9(-10)$ & $6.0(-11)$ & $3.6(-9)$ \\
\hline$N(\mathrm{HNC})$ & 7.1(13) & $4.8(13)$ & $9.4(13)$ & $3.7(12)$ & 4.4(14) \\
\hline$x(\mathrm{HNC})$ & $2.2(-9)$ & $1.6(-9)$ & $2.1(-9)$ & $2.3(-10)$ & $8.4(-9)$ \\
\hline$N\left(\mathrm{HC}_{3} \mathrm{~N}\right)$ & $1.6(14)$ & $2.8(13)$ & $2.8(14)$ & $7.0(12)$ & 7.9(14) \\
\hline$x\left(\mathrm{HC}_{3} \mathrm{~N}\right)$ & $5.0(-9)$ & $6.1(-10)$ & $8.5(-9)$ & $1.0(-10)$ & $2.7(-8)$ \\
\hline$N\left(\mathrm{CH}_{3} \mathrm{CN}\right)^{b}$ & $5.5(11)$ & $5.4(11)$ & $1.4(11)$ & $4.2(11)$ & $7.0(11)$ \\
\hline$x\left(\mathrm{CH}_{3} \mathrm{CN}\right)^{b}$ & $1.1(-11)$ & $1.0(-11)$ & $8.1(-12)$ & $4.0(-12)$ & $2.0(-11)$ \\
\hline$N\left(\mathrm{~N}_{2} \mathrm{H}^{+}\right)$ & $5.4(13)$ & $2.8(13)$ & 8.8(13) & $6.6(12)$ & $5.4(14)$ \\
\hline$x\left(\mathrm{~N}_{2} \mathrm{H}^{+}\right)$ & $1.6(-9)$ & $9.9(-10)$ & $1.8(-9)$ & $2.8(-10)$ & $9.8(-9)$ \\
\hline \multicolumn{6}{|c|}{ IR-dark clumps } \\
\hline Quantity & Mean & Median & $\mathrm{Std}^{a}$ & Min. & Max. \\
\hline$N(\mathrm{SiO})$ & $1.5(13)$ & $1.1(13)$ & $7.9(12)$ & $8.2(12)$ & $2.8(13)$ \\
\hline$x(\mathrm{SiO})$ & $3.6(-10)$ & $3.5(-10)$ & $1.4(-10)$ & $1.6(-10)$ & $5.7(-10)$ \\
\hline$N\left(\mathrm{HN}^{13} \mathrm{C}\right)$ & $9.6(12)^{c}$ & & & & \\
\hline$x\left(\mathrm{HN}^{13} \mathrm{C}\right)$ & $1.0(-10)^{c}$ & & & & \\
\hline$N\left(\mathrm{C}_{2} \mathrm{H}\right)$ & $4.5(14)$ & $1.7(14)$ & $4.7(14)$ & $7.8(13)$ & $1.2(15)$ \\
\hline$x\left(\mathrm{C}_{2} \mathrm{H}\right)$ & $8.6(-9)$ & $6.3(-9)$ & $4.0(-9)$ & $5.1(-9)$ & $1.3(-8)$ \\
\hline$N(\mathrm{HNCO})$ & $2.4(14)$ & $1.3(14)$ & $2.7(14)$ & $5.4(12)$ & $7.9(14)$ \\
\hline$x(\mathrm{HNCO})$ & $5.5(-9)$ & 4.1(-9) & $5.7(-9)$ & $1.3(-10)$ & $1.9(-8)$ \\
\hline$N(\mathrm{HCN})$ & $6.5(14)$ & $2.0(14)$ & $7.5(14)$ & $3.4(13)$ & $1.7(15)$ \\
\hline$x(\mathrm{HCN})$ & $2.0(-8)$ & $4.4(-9)$ & $2.5(-8)$ & $9.3(-10)$ & $5.6(-8)$ \\
\hline$N\left(\mathrm{HCO}^{+}\right)$ & $2.2(13)$ & $1.3(13)$ & 2.1(13) & $1.4(12)$ & $6.3(13)$ \\
\hline$x\left(\mathrm{HCO}^{+}\right)$ & $4.3(-10)$ & $3.5(-10)$ & $3.3(-10)$ & $6.0(-11)$ & $1.1(-9)$ \\
\hline$N(\mathrm{HNC})$ & $9.6(13)$ & $6.3(13)$ & $1.4(14)$ & $3.7(12)$ & $5.3(14)$ \\
\hline$x(\mathrm{HNC})$ & $2.0(-9)$ & $1.7(-9)$ & $1.5(-9)$ & $4.2(-10)$ & $5.5(-9)$ \\
\hline$N\left(\mathrm{HC}_{3} \mathrm{~N}\right)$ & $1.6(14)$ & $3.3(13)$ & $3.1(14)$ & $1.4(13)$ & $7.9(14)$ \\
\hline$x\left(\mathrm{HC}_{3} \mathrm{~N}\right)$ & $5.1(-9)$ & $5.7(-10)$ & $1.1(-8)$ & $2.8(-10)$ & $2.7(-8)$ \\
\hline$N\left(\mathrm{CH}_{3} \mathrm{CN}\right)$ & $5.6(11)$ & $5.6(11)$ & $2.0(11)$ & $4.2(11)$ & $7.0(11)$ \\
\hline$x\left(\mathrm{CH}_{3} \mathrm{CN}\right)$ & $1.2(-11)$ & $1.2(-11)$ & $1.1(-11)$ & $4.0(-12)$ & $2.0(-11)$ \\
\hline$N\left(\mathrm{~N}_{2} \mathrm{H}^{+}\right)$ & $9.0(13)$ & $4.0(13)$ & $1.6(14)$ & $1.6(13)$ & $5.4(14)$ \\
\hline$x\left(\mathrm{~N}_{2} \mathrm{H}^{+}\right)$ & $1.8(-9)$ & $1.3(-9)$ & $1.5(-9)$ & $7.3(-10)$ & $5.6(-9)$ \\
\hline \multicolumn{6}{|c|}{ IR-bright clumps } \\
\hline Quantity & Mean & Median & $\mathrm{Std}^{a}$ & Min. & Max. \\
\hline$N\left(\mathrm{H}^{13} \mathrm{CO}^{+}\right)$ & $7.4(12)$ & $6.4(12)$ & $2.3(12)$ & $5.8(12)$ & $1.0(13)$ \\
\hline$x\left(\mathrm{H}^{13} \mathrm{CO}^{+}\right)$ & $9.7(-11)$ & $1.1(-10)$ & $5.1(-11)$ & $4.0(-11)$ & $1.4(-10)$ \\
\hline$N(\mathrm{SiO})$ & $2.4(13)$ & $2.2(13)$ & $1.8(13)$ & $5.5(12)$ & $4.8(13)$ \\
\hline$x(\mathrm{SiO})$ & $9.4(-10)$ & $9.7(-10)$ & $7.3(-10)$ & $4.0(-11)$ & $1.8(-9)$ \\
\hline$N\left(\mathrm{HN}^{13} \mathrm{C}\right)$ & $1.2(13)$ & $1.1(13)$ & $2.6(12)$ & $1.0(13)$ & $1.5(13)$ \\
\hline$x\left(\mathrm{HN}^{13} \mathrm{C}\right)$ & $1.3(-10)$ & $1.2(-10)$ & $1.2(-11)$ & $1.2(-10)$ & $1.4(-10)$ \\
\hline$N\left(\mathrm{C}_{2} \mathrm{H}\right)$ & 4.2(14) & $4.0(14)$ & $2.3(14)$ & $1.1(14)$ & $8.8(14)$ \\
\hline$x\left(\mathrm{C}_{2} \mathrm{H}\right)$ & $7.9(-9)$ & $5.2(-9)$ & $7.3(-9)$ & $1.8(-9)$ & $2.4(-8)$ \\
\hline$N(\mathrm{HNCO})$ & $1.8(14)$ & $1.5(14)$ & $1.3(14)$ & $1.7(13)$ & $4.3(14)$ \\
\hline$x(\mathrm{HNCO})$ & $7.9(-9)$ & $6.2(-9)$ & $5.9(-9)$ & $6.1(-10)$ & $1.9(-8)$ \\
\hline$N(\mathrm{HCN})$ & $5.7(14)$ & $6.1(13)$ & $1.6(15)$ & $1.6(13)$ & $5.5(15)$ \\
\hline$x(\mathrm{HCN})$ & $2.1(-8)$ & $1.7(-9)$ & $6.1(-8)$ & $2.7(-10)$ & $2.1(-7)$ \\
\hline$N\left(\mathrm{HCO}^{+}\right)$ & $3.3(13)$ & $9.2(12)$ & $6.5(13)$ & $1.1(12)$ & $2.6(14)$ \\
\hline$x\left(\mathrm{HCO}^{+}\right)$ & $6.2(-10)$ & $3.2(-10)$ & $8.0(-10)$ & $7.0(-11)$ & $3.6(-9)$ \\
\hline$N(\mathrm{HNC})$ & $7.7(13)$ & $3.3(13)$ & 1.1(14) & $4.0(12)$ & $4.4(14)$ \\
\hline$x(\mathrm{HNC})$ & $2.4(-9)$ & $1.4(-9)$ & $2.4(-9)$ & $2.3(-10)$ & $8.4(-9)$ \\
\hline$N\left(\mathrm{HC}_{3} \mathrm{~N}\right)$ & $1.6(14)$ & $2.8(13)$ & $2.7(14)$ & $7.0(12)$ & $7.6(14)$ \\
\hline$x\left(\mathrm{HC}_{3} \mathrm{~N}\right)$ & $4.7(-9)$ & $7.8(-10)$ & $7.2(-9)$ & $1.0(-10)$ & $1.8(-8)$ \\
\hline$N\left(\mathrm{CH}_{3} \mathrm{CN}\right)$ & $5.4(11)^{c}$ & & & & \\
\hline$x\left(\mathrm{CH}_{3} \mathrm{CN}\right)$ & $1.0(-11)^{c}$ & & & & \\
\hline$N\left(\mathrm{~N}_{2} \mathrm{H}^{+}\right)$ & 4.1(13) & $2.5(13)$ & $3.7(13)$ & $6.6(12)$ & $1.4(14)$ \\
\hline$x\left(\mathrm{~N}_{2} \mathrm{H}^{+}\right)$ & $1.5(-9)$ & $9.0(-10)$ & $1.9(-9)$ & $2.8(-10)$ & $9.8(-9)$ \\
\hline
\end{tabular}

Notes. The notation $a(b)$ means $a \times 10^{b} .{ }^{(a)}$ Standard deviation. ${ }^{(b)}$ Only three detections. ${ }^{(c)}$ Only one detection.

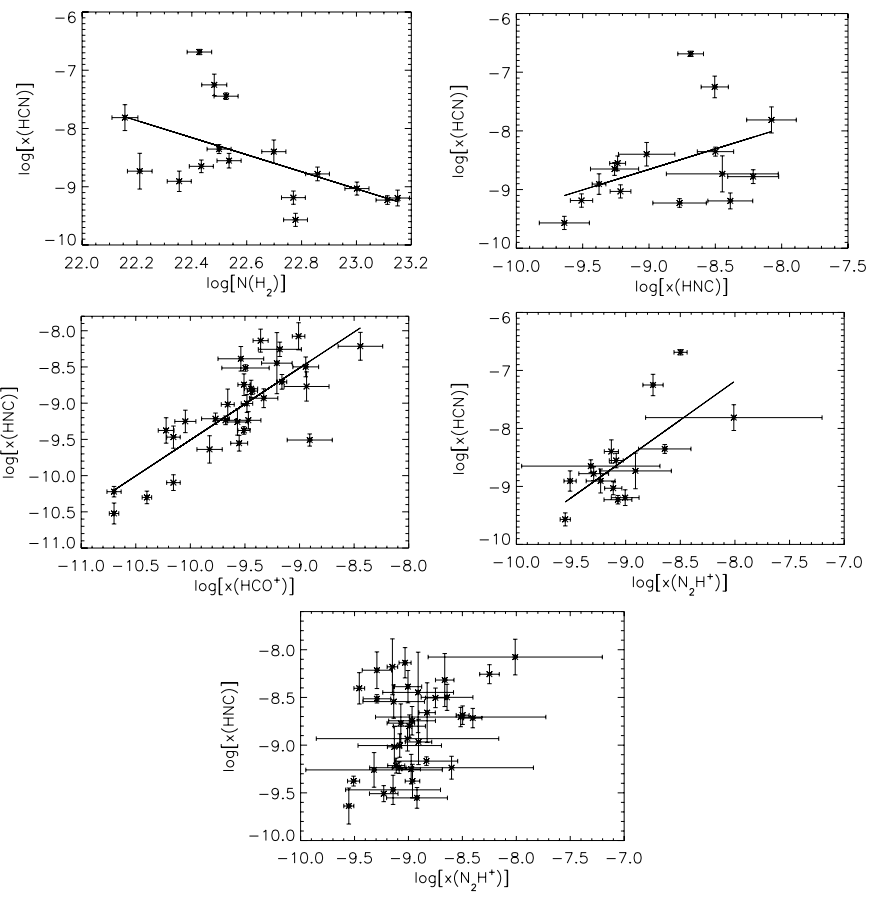

Fig. 2. Top left panel: HCN fractional abundance plotted as a function of $\mathrm{H}_{2}$ column density in logarithmic scales. The rest of the panels show the correlations found between the derived fractional abundances of the molecules. From top right to bottom panel, the panels plot $x(\mathrm{HCN})$ versus $x(\mathrm{HNC}), x(\mathrm{HNC})$ versus $x\left(\mathrm{HCO}^{+}\right), x(\mathrm{HCN})$ versus $x\left(\mathrm{~N}_{2} \mathrm{H}^{+}\right)$, and $x(\mathrm{HNC})$ versus $x\left(\mathrm{~N}_{2} \mathrm{H}^{+}\right)$in logarithmic scales. The solid lines show the least squares fit to the data (see text for details).

obtained by VLH11 and SJF12, because they also employed the Mopra telescope observations for their studies.

\section{1. $\mathrm{HCO}^{+}$and $\mathrm{H}^{13} \mathrm{CO}^{+}$(formylium)}

In dense molecular clouds, $\mathrm{HCO}^{+}$is mainly formed through the gas-phase ion-neutral reaction $\mathrm{H}_{3}^{+}+\mathrm{CO} \rightarrow \mathrm{HCO}^{+}+\mathrm{H}_{2}$ (e.g. Herbst \& Klemperer 1973). The $\mathrm{HCO}^{+}$abundance can be increased in regions where shocks are generated, e.g. due to outflows from embedded YSOs. When the shock heats the gas and produces UV radiation through Ly- $\alpha$ emission $(\lambda=121.6 \mathrm{~nm})$, the icy grain mantles evaporate and the $\mathrm{HCO}^{+}$abundance is enhanced because of evaporated $\mathrm{CO}$ and $\mathrm{H}_{2} \mathrm{O}$, where the latter species can form $\mathrm{HCO}^{+}$in the reaction with photoionised carbon $\left(\mathrm{C}^{+}+\mathrm{H}_{2} \mathrm{O} \rightarrow \mathrm{HCO}^{+}+\mathrm{H}\right.$; Rawlings et al. 2000, 2004). The destruction of the $\mathrm{HCO}^{+}$molecules is, in turn, mainly caused by the dissociative recombination with electrons, $\mathrm{HCO}^{+}+\mathrm{e}^{-} \rightarrow$ $\mathrm{CO}+\mathrm{H}$.

Extended $\mathrm{HCO}^{+}$emission is seen particularly around the submm peaks G1.87-SMM 1, 8, 10, 12, 14-16, and G13.22-SMM 4, 5, 6, 7, 10, 11, 23, 27, and 32. Moreover, the submm clump/UC H II region G2.11-SMM 5 is associated with an elongated $\mathrm{HCO}^{+}$clump (Fig. B.8). In the case of G2.11-SMM 5, G11.36-SMM 5, G13.22-SMM 5, and $\mathrm{G} 13.22-\mathrm{SMM} 32$, the $\mathrm{HCO}^{+}$emission peak is close to the LABOCA $870 \mu \mathrm{m}$ dust emission peak. The clump G1.87-SMM 1 is classified as IR-dark, but the $\mathrm{HCO}^{+}$line towards the submm peak position shows nonGaussian wing emission, indicative of outflows/shocks. The clumps G13.22-SMM 4-7 and 11 are associated with the Spitzer IR-bubble system N10/11 (Figs. B.10 and B.11; 
Table 5. Column density ratios.

\begin{tabular}{|c|c|c|c|c|c|}
\hline Source & $\frac{N(\mathrm{HNC})}{N(\mathrm{HCN})}$ & $\frac{N\left(\mathrm{HNC}^{+}\right.}{N\left(\mathrm{HCO}^{+}\right)}$ & $\frac{N\left(\mathrm{~N}_{2} \mathrm{H}^{+}\right)}{N\left(\mathrm{HCO}^{+}\right)}$ & $\frac{N\left(\mathrm{~N}_{2} \mathrm{H}^{+}\right)}{N(\mathrm{HNC})}$ & $\frac{N\left(\mathrm{HC}_{3} \mathrm{~N}\right)}{N(\mathrm{HCN})}$ \\
\hline \multicolumn{6}{|l|}{ G1.87-0.14 } \\
\hline SMM 1 & $\ldots$ & $8.41 \pm 4.13$ & $8.57 \pm 4.13$ & $1.02 \pm 0.28$ & $\ldots$ \\
\hline SMM $1^{a}$ & $\ldots$ & $1.83 \pm 0.06$ & $\ldots$ & & $\ldots$ \\
\hline SMM 8 & $0.71 \pm 0.24$ & $2.78 \pm 1.08$ & $2.00 \pm 1.19$ & $0.72 \pm 0.45$ & $\ldots$ \\
\hline SMM 10 & $0.55 \pm 0.36$ & $8.57 \pm 3.62$ & $10.00 \pm 18.59$ & $1.17 \pm 2.22$ & $\ldots$ \\
\hline SMM 12 & $\ldots$ & $2.84 \pm 0.61$ & $4.40 \pm 8.00$ & $1.55 \pm 2.84$ & $\ldots$ \\
\hline SMM 14 & $\ldots$ & $5.87 \pm 1.97$ & $3.53 \pm 2.41$ & $0.60 \pm 0.45$ & $\ldots$ \\
\hline SMM 15 & $\ldots$ & $\ldots$ & $\ldots$ & $0.11 \pm 0.07$ & $\ldots$ \\
\hline SMM 20 & $\ldots$ & $\ldots$ & $\ldots$ & $0.09 \pm 0.03$ & $\ldots$ \\
\hline SMM 21 & $\ldots$ & $\ldots$ & $\ldots$ & $2.05 \pm 0.55$ & $\ldots$ \\
\hline SMM 23 & $0.24 \pm 0.16$ & $4.36 \pm 2.13$ & $3.36 \pm 0.48$ & $0.77 \pm 0.38$ & $0.07 \pm 0.03$ \\
\hline SMM $23^{a}$ & $\ldots$ & $\ldots$ & $\ldots$ & $1.74 \pm 1.15$ & $\ldots$ \\
\hline SMM 24 & $\ldots$ & $\ldots$ & $\ldots$ & $0.26 \pm 0.19$ & $\ldots$ \\
\hline SMM 27 & $0.06 \pm 0.03$ & $\ldots$ & $\ldots$ & $0.57 \pm 0.16$ & $0.02 \pm 0.01$ \\
\hline SMM 28 & $0.01 \pm 0.002$ & $\ldots$ & $\ldots$ & $1.55 \pm 0.34$ & $0.02 \pm 0.01$ \\
\hline SMM 30 & $\ldots$ & $4.36 \pm 1.16$ & $2.82 \pm 0.94$ & $0.65 \pm 0.26$ & $\ldots$ \\
\hline SMM 31 & $\ldots$ & $16.49 \pm 6.13$ & $2.11 \pm 0.31$ & $0.13 \pm 0.05$ & $\ldots$ \\
\hline SMM 38 & $\ldots$ & $\ldots$ & $\ldots$ & $0.69 \pm 0.51$ & $\ldots$ \\
\hline SMM $38^{a}$ & $\ldots$ & $\ldots$ & $\ldots$ & $0.48 \pm 0.31$ & $\ldots$ \\
\hline \multicolumn{6}{|l|}{$\mathrm{G} 2.11+0.00$} \\
\hline SMM 5 & $0.25 \pm 0.11$ & $2.03 \pm 0.90$ & $1.76 \pm 2.59$ & $0.87 \pm 1.31$ & $\ldots$ \\
\hline \multicolumn{6}{|l|}{$\mathrm{G} 11.36+0.80$} \\
\hline SMM 1 & $\ldots$ & $5.94 \pm 2.19$ & $11.25 \pm 2.34$ & $1.89 \pm 0.73$ & $\ldots$ \\
\hline SMM 2 & $\ldots$ & $7.14 \pm 3.03$ & $18.57 \pm 3.41$ & $2.60 \pm 1.08$ & $\ldots$ \\
\hline SMM 3 & $\ldots$ & $5.18 \pm 1.97$ & $10.91 \pm 11.09$ & $2.11 \pm 2.22$ & $\ldots$ \\
\hline SMM 4 & $\ldots$ & $\ldots$ & & $1.15 \pm 0.69$ & $\ldots$ \\
\hline SMM 5 & $\ldots$ & $1.00 \pm 0.26$ & $4.25 \pm 2.80$ & $4.25 \pm 2.91$ & $\ldots$ \\
\hline SMM 6 & $\ldots$ & $1.68 \pm 0.56$ & $7.27 \pm 12.83$ & $4.32 \pm 7.64$ & $\ldots$ \\
\hline SMM 7 & $\ldots$ & $2.47 \pm 0.98$ & $2.06 \pm 4.07$ & $0.84 \pm 1.65$ & $\ldots$ \\
\hline \multicolumn{6}{|l|}{ G13.22-0.06 } \\
\hline SMM 4 & $0.21 \pm 0.06$ & $2.78 \pm 0.71$ & $3.89 \pm 1.01$ & $1.40 \pm 0.21$ & \\
\hline SMM 5 & $2.86 \pm 1.35$ & $1.47 \pm 0.96$ & $0.73 \pm 0.40$ & $0.50 \pm 0.27$ & $0.17 \pm 0.03$ \\
\hline SMM 6 & $0.34 \pm 0.13$ & $1.36 \pm 0.13$ & $1.00 \pm 0.09$ & $0.73 \pm 0.36$ & $\ldots$ \\
\hline SMM 7 & $6.38 \pm 3.05$ & $14.23 \pm 8.49$ & $3.42 \pm 1.85$ & $0.24 \pm 0.11$ & $\ldots$ \\
\hline $\operatorname{SMM} 7\left(37 \mathrm{~km} \mathrm{~s}^{-1}\right)^{b}$ & $\ldots$ & $3.67 \pm 0.83$ & & & $\ldots$ \\
\hline SMM 11 & $\ldots$ & $3.02 \pm 0.83$ & $2.54 \pm 2.23$ & $0.84 \pm 0.77$ & $\ldots$ \\
\hline SMM $11\left(37 \mathrm{~km} \mathrm{~s}^{-1}\right)^{b}$ & $\ldots$ & $1.07 \pm 0.26$ & & & $\ldots$ \\
\hline SMM 23 & $1.93 \pm 2.31$ & $5.80 \pm 5.86$ & $2.00 \pm 1.62$ & $0.34 \pm 0.42$ & $\ldots$ \\
\hline SMM $23\left(53 \mathrm{~km} \mathrm{~s}^{-1}\right)^{b}$ & & $1.68 \pm 0.34$ & & & $\ldots$ \\
\hline SMM 27 & $3.67 \pm 1.83$ & $1.69 \pm 1.07$ & $0.14 \pm 0.08$ & $0.08 \pm 0.04$ & $0.06 \pm 0.02$ \\
\hline SMM 29 & $0.88 \pm 0.43$ & $1.52 \pm 0.76$ & $1.85 \pm 0.47$ & $1.21 \pm 0.52$ & $\ldots$ \\
\hline SMM $29\left(13.6 \mathrm{~km} \mathrm{~s}^{-1}\right)^{b}$ & $\ldots$ & $1.68 \pm 0.43$ & $\ldots$ & $\ldots$ & $\ldots$ \\
\hline SMM $29\left(37 \mathrm{~km} \mathrm{~s}^{-1}\right)^{b}$ & $\ldots$ & $1.25 \pm 0.17$ & $\ldots$ & $\ldots$ & $\ldots$ \\
\hline SMM $29^{a}$ & $\ldots$ & $1.55 \pm 1.11$ & $2.58 \pm 1.44$ & $1.67 \pm 1.00$ & $\ldots$ \\
\hline SMM 32 & $0.47 \pm 0.14$ & $0.25 \pm 0.12$ & $0.48 \pm 0.26$ & $1.94 \pm 0.64$ & $0.24 \pm 0.06$ \\
\hline SMM $32^{a}$ & $0.38 \pm 0.11$ & $1.40 \pm 0.43$ & $4.95 \pm 1.53$ & $3.54 \pm 0.77$ & $\ldots$ \\
\hline SMM $32\left(14 \mathrm{~km} \mathrm{~s}^{-1}\right)^{a, b}$ & $\ldots$ & $1.83 \pm 0.51$ & $\ldots$ & $\ldots$ & $\ldots$ \\
\hline
\end{tabular}

Notes. ${ }^{(a)}$ Towards the line emission peak. ${ }^{(b)}$ For the additional velocity component.

Churchwell et al. 2006) that, it has been suggested, represents a site of triggered massive-star formation (Watson et al. 2008). The strong $\mathrm{HCO}^{+}$emission seen towards the bubble surroundings could originate in the swept-up bubble shells where shock fronts are expanding into the surrounding medium. Moreover, the high-mass stars in the system produce a strong radiation field of UV photons. Some of the IRDCs studied by Liu et al. (2013) show similar extended $\mathrm{HCO}^{+}$emission to the sources studied here. The $\mathrm{HCO}^{+}$column densities derived by Liu et al. (2013), $2.24 \times 10^{12}-1.31 \times 10^{13} \mathrm{~cm}^{-2}\left(\sim 5.1 \times 10^{12} \mathrm{~cm}^{-2}\right.$ on average), are lower by a factor of about six on average than those we derived. On the other hand, the values derived by SJF12, $5.8 \times 10^{13}-1 \times 10^{15} \mathrm{~cm}^{-2}$ with the median of $1.88 \times 10^{14} \mathrm{~cm}^{-2}$, exceed our values.
The $\mathrm{HCO}^{+}$abundances we derive lie in the range $6 \times$ $10^{-11}-3.6 \times 10^{-9}$, with the mean (median) value of $5.6 \times 10^{-10}$ $\left(3.3 \times 10^{-10}\right)$. For their sample of the 4 th quadrant IRDC sources, VLH1 1 derived the abundances of $3.5 \times 10^{-9}-5.1 \times 10^{-8}$ with an average value of $1.7 \times 10^{-8}$. The values determined by SJF12 for $x\left(\mathrm{HCO}^{+}\right)$for their sample of IRDC clumps were also higher, ranging from $3.9 \times 10^{-9}$ to $2.8 \times 10^{-7}$ (with the median of $2.51 \times 10^{-8}$ ). These authors found that both the $\mathrm{HCO}^{+}$column density and abundance increase as the clump evolves from the quiescent state with no IR emission (as seen by Spitzer) to the red state with bright $8 \mu \mathrm{m}$ emission and when the central source has probably formed an H II region. More recently, the MALT90 study of 333 massive clumps by Hoq et al. (2013) revealed a similar evolutionary trend in $x\left(\mathrm{HCO}^{+}\right)$(their Fig. 5). We also 
derive higher $\mathrm{HCO}^{+}$column densities and abundances on average for IR-bright clumps than for IR-dark ones, although the median values are quite similar between the two classes. The lowest value of $x\left(\mathrm{HCO}^{+}\right)$in our sample is derived towards the IR-dark clump SMM 2 in the G11.36 filament, while the highest abundance is seen towards the IR-bright clump G13.22-SMM 27. The G13.22-SMM 32 clump, associated with an HII region, also shows a relatively high value of $x\left(\mathrm{HCO}^{+}\right)$compared to the rest of our sources. These findings are in agreement with the evolutionary trend found by SJF12. The fact that we derive lower values of $x\left(\mathrm{HCO}^{+}\right)$than in VLH11 and SJF12 could mean that our sources are, on average, less evolved. The depletion of $\mathrm{CO}$ molecules would, at least partly, explain the meagre amount of $\mathrm{HCO}^{+}$found in the present study. In Paper I, the CO depletion factor was derived towards some of our clumps. For example, towards G11.36-SMM 1 and G13.22-SMM 27 the values $f_{\mathrm{D}}(\mathrm{CO})=3.9 \pm 0.6$ and $9.9 \pm 1.5$ were determined

The ${ }^{13} \mathrm{C}$ isotopologue $\mathrm{H}^{13} \mathrm{CO}^{+}$is formed in a similar way to the main ${ }^{12} \mathrm{C}$-form except from ${ }^{13} \mathrm{CO}$. The isotope transfer via $\mathrm{HCO}^{+}+{ }^{13} \mathrm{CO} \leftrightarrow \mathrm{H}^{13} \mathrm{CO}^{+}+{ }^{12} \mathrm{CO}$ can also play a role in the formation of $\mathrm{H}^{13} \mathrm{CO}^{+}$(Langer et al. 1984). Only weak emission of $\mathrm{H}^{13} \mathrm{CO}^{+}$, if any, is detected towards our clumps (only three detections). Towards G13.22-SMM 27, the line emission is quite well correlated with the $8 \mu \mathrm{m}$ absorption (Fig. B.12). The column densities and fractional abundances are derived to be $5.8 \times 10^{12}-1 \times 10^{13} \mathrm{~cm}^{-2}\left(7.4 \times 10^{12} \mathrm{~cm}^{-2}\right.$ on average $)$ and $4 \times 10^{-11}-1.4 \times 10^{-10}\left(9.7 \times 10^{-11}\right.$ on average $)$. The former values are comparable to those found by Sakai et al. (2010) for their sample of clumps within IRDCs $\left(1.3 \times 10^{12}-1.4 \times 10^{13} \mathrm{~cm}^{-2}\right)$. Vasyunina et al. (2011) derived $\mathrm{H}^{13} \mathrm{CO}^{+}$abundances of $7.6 \times$ $10^{-11}-7.4 \times 10^{-10}$ with an average of $3.4 \times 10^{-10}$, which is 3.5 times higher than our average abundance.

\subsection{SiO (silicon monoxide)}

In star-forming regions, $\mathrm{SiO}$ emission is usually believed to be linked to the action of high-velocity $\left(\sim 20-50 \mathrm{~km} \mathrm{~s}^{-1}\right)$ shocks (Martín-Pintado et al. 1992; Schilke et al. 1997; Gusdorf et al. 2008a,b). SiO emission can also trace irradiated mediumvelocity $\left(\sim 10-20 \mathrm{~km} \mathrm{~s}^{-1}\right)$ shocks in PDRs (e.g. Schilke et al. 2001).

The compund $\mathrm{SiO}$ can form via sputtering of $\mathrm{Si}$ atoms from the grain cores, which then undergo oxidation through the neutral-neutral gas-phase reactions $\mathrm{Si}+\mathrm{O}_{2} \rightarrow \mathrm{SiO}+\mathrm{O}$ and $\mathrm{Si}+\mathrm{OH} \rightarrow \mathrm{SiO}+\mathrm{H}$. If $\mathrm{Si}$ is present in the icy grain mantles, a clearly lower shock velocity is sufficient to release it into the gas phase (Gusdorf et al. 2008b). Alternatively, SiO can be directly formed through dust destruction by vaporisation in grain-grain collisions (e.g. Guillet et al. 2009). In the hot postshock gas, $\mathrm{OH}$ molecules are abundant owing to the reaction $\mathrm{O}+\mathrm{H}_{2} \rightarrow \mathrm{OH}+\mathrm{O}$. When $\mathrm{SiO}$ reacts with $\mathrm{OH}$, a conversion to $\mathrm{SiO}_{2}$ takes place ( $\mathrm{SiO}+\mathrm{OH} \rightarrow \mathrm{SiO}_{2}+\mathrm{H}$; Schilke et al. 1997). This limits the $\mathrm{SiO}$ abundance in the shocked gas.

The $\mathrm{SiO}$ emission appears to be quite extended, particularly towards G1.87-SMM 27, 28, 31 (Fig. B.6). Moreover, G1.87-SMM 20, 23, 30 (Fig. B.5) and G1.87-SMM 38 (Fig. B.7) are associated with a few parsec-scale $\mathrm{SiO}$ clump. It is also worth noting that the submm peaks G1.87-SMM 28, 30 , and 38 are coincident with the local $\mathrm{SiO}$ peak positions. Jiménez-Serra et al. (2010) proposed that the extended SiO emission they observed along the filamentary IRDC G035.39-00.33 is the result of a low-velocity shock produced by colliding flows (see also Henshaw et al. 2013). The widespread SiO emission could therefore originate in the cloud formation process instead of during star formation. Sanhueza et al. (2013) recently detected $\mathrm{SiO}$ emission from the candidate starless IRDC G028.23-00.19. The authors suggested that the $\mathrm{SiO}$ emission with narrow linewidths, coincident with the subclouds' interface within the source, could be caused by vaporisation of icy grain mantles in grain-grain collisions. Some of our clumps around which extended $\mathrm{SiO}$ emission is detected are, however, associated with IR sources, and probably host embedded YSOs. Outflows from these forming stars are likely to be responsible for the detected $\mathrm{SiO}$ emission. This is supported by the fact that the $\mathrm{SiO}$ and LABOCA emission peaks are coincident in G1.87-SMM 28, 30 , and 38 , and that some of the line profiles show wing emission. We also note that the filamentary IRDC G11.36 does not show extended $\mathrm{SiO}$ emission along its long axis (and neither do the other filaments in this study). The clumps SMM 4 and 5 around the bubble system N10/11 are not associated with extended $\mathrm{SiO}$ emission, although expanding shock fronts are expected to be present there.

Sakai et al. (2010) found that towards their IRDC sources, the $\mathrm{SiO}$ column densities are $\sim 4.6 \times 10^{12}-3.8 \times 10^{13} \mathrm{~cm}^{-2}$ with an average value of $1.5 \times 10^{13} \mathrm{~cm}^{-2}$. This is very similar to our clumps, for which $N(\mathrm{SiO})=5.5 \times 10^{12}-4.8 \times 10^{13} \mathrm{~cm}^{-2}$ $\left(1.9 \times 10^{13} \mathrm{~cm}^{-2}\right.$ on average). However, given the assumption made for $T_{\text {ex }}$ (Sect. 3.3), our $N(\mathrm{SiO})$ values should be taken as lower limits. Vasyunina et al. (2011) derived $\mathrm{SiO}$ abundances in the range of $1.6 \times 10^{-10}-1.6 \times 10^{-9}$ (average value $9.5 \times$ $\left.10^{-10}\right)$ towards their IRDCs. Our values, $4 \times 10^{-11}-1.8 \times 10^{-9}$ (average $5.7 \times 10^{-10}$ ), are mostly comparable to them. Sanhueza et al. (2012) found that the $\mathrm{SiO}$ column densities and abundances for their whole sample are $1.36 \times 10^{12}-3.47 \times 10^{13} \mathrm{~cm}^{-2}$ and $2.78 \times 10^{-10}-9.20 \times 10^{-10}$ (median values are $7.72 \times 10^{12} \mathrm{~cm}^{-2}$ and $\left.5.79 \times 10^{-10}\right)$. In particular, their median abundance is close to what we found $\left(3.6 \times 10^{-10}\right)$.

The mean and median $\mathrm{SiO}$ abundances are found to be almost three times higher towards IR-bright clumps than towards IR-dark sources (Table 4), which is in accordance with the results by SJF12. However, there are distinct exceptions from the average $\mathrm{SiO}$ trend among our sources, calling its statistical significance into question. The lowest $\mathrm{SiO}$ abundance is derived towards the IR-bright clump G13.22-SMM 5, and the second highest $\mathrm{SiO}$ abundance is observed towards the IR-dark clump G1.87-SMM 31. Indeed, Sakai et al. (2010) found a trend opposite to ours, and they proposed that the $\mathrm{SiO}$ emission from the mid-IR dark sources originates in newly formed shocks, while the $\mathrm{SiO}$ emission from more evolved, mid-IR bright sources could originate in gas that was shocked earlier in time. This could be related to the discovery by Miettinen et al. (2006), namely that the $\mathrm{SiO}$ abundance in massive clumps appears to decrease as a function of gas kinetic temperature, which might reflect an evolutionary trend.

\section{3. $\mathrm{HCN}, \mathrm{HNC}$, and $H N^{13} \mathrm{C}$ [hydrogen (iso-)cyanide]}

Gas-phase chemical models suggest that $\mathrm{HCN}$ and its metastable geometrical isomer, HNC (hydrogen isocyanide), are primarily produced via the dissociative recombination reaction $\mathrm{HCNH}^{+}+$ $\mathrm{e}^{-} \rightarrow \mathrm{HCN}+\mathrm{H}$ or $\mathrm{HNC}+\mathrm{H}$ (e.g. Herbst 1978). The resulting $\mathrm{HNC} / \mathrm{HCN}$ abundance ratio is predicted to be 0.9 in this case, i.e. close to unity. The formation of HNC (and only HNC, not $\mathrm{HCN}$ ) can also take place via the reactions $\mathrm{H}_{2} \mathrm{CN}^{+}+\mathrm{e}^{-}$ or $\mathrm{H}_{2} \mathrm{NC}^{+}+\mathrm{e}^{-} \rightarrow \mathrm{HNC}+\mathrm{H}$ (Pearson \& Schaefer 1974; Allen et al. 1980). As a result of this additional HNC production channel, the $\mathrm{HNC} / \mathrm{HCN}$ ratio can rise above unity. Other ways to form $\mathrm{HCN}$ and $\mathrm{HNC}$ are the neutral-neutral reactions 
$\mathrm{CH}_{2}+\mathrm{N} \rightarrow \mathrm{HCN}+\mathrm{H}$ and $\mathrm{NH}_{2}+\mathrm{C} \rightarrow \mathrm{HNC}+\mathrm{H}$ (Herbst et al. 2000). After these reactions, the species are able to undergo rapid isomerisation reactions, again leading to the near unity $\mathrm{HNC} / \mathrm{HCN}$ ratio.

In dense clouds and PDRs, HCN can be photodissociated into $\mathrm{CN}$, either directly or via cosmic-ray induced photodissociation (Boger \& Sternberg 2005). Additional destruction processes of $\mathrm{HCN}$ in dense clouds are $\mathrm{HCN}+\mathrm{H}^{+} \rightarrow \mathrm{HCN}^{+}+\mathrm{H}$ and $\mathrm{HCN}+\mathrm{HCO}^{+} \rightarrow \mathrm{H}_{2} \mathrm{CN}^{+}+\mathrm{CO}$ (Boger \& Sternberg 2005). The $\mathrm{HNC}$ molecule can be destroyed via the reactions with hydrogen and oxygen atoms, $\mathrm{HNC}+\mathrm{H} \rightarrow \mathrm{HCN}+\mathrm{H}$ and $\mathrm{HNC}+\mathrm{O} \rightarrow$ $\mathrm{NH}+\mathrm{CO}$ (Schilke et al. 1992; Talbi et al. 1996). HNC also converts to $\mathrm{HCN}$ through the reaction $\mathrm{HNC}+\mathrm{H}^{+} \rightarrow \mathrm{HCN}+\mathrm{H}^{+}$ (Herbst et al. 2000).

As can be seen in the 0th moment maps in Appendix B, the spatial distributions of $\mathrm{HCN}$ and $\mathrm{HNC}$ emissions are generally extended. Moreover, for example in the case of G2.11-SMM 5 and G13.22-SMM 5, the submm peak position is close to the peak $\mathrm{HCN}$ and $\mathrm{HNC}$ emissions. The $\mathrm{HN}^{13} \mathrm{C}$ emission is extended, although weak, in G1.87-SMM 20, 23, and 30 (Fig. B.5). Similarly, Jackson et al. (2010) found that HNC(1-0) emission traces well the filamentary IRDC G338.4-0.4, also known as the Nessie Nebula. Sanhueza et al. (2012) found that HNC is ubiquitous in the clumps of IRDCs, in agreement with our results. Liu et al. (2013) also found a good correlation between the $8 \mu \mathrm{m}$ absorption and $\mathrm{HNC}$ and $\mathrm{HCN}$ emissions towards many of their IRDCs.

The $\mathrm{HCN}$ and $\mathrm{HNC}$ abundances we derived are in the ranges of $2.7 \times 10^{-10}-2.1 \times 10^{-7}$ and $2.3 \times 10^{-10}-8.4 \times 10^{-9}$, respectively. The $\mathrm{HN}^{13} \mathrm{C}$ abundances are found to be $\sim 10^{-10}$ on average. Sakai et al. (2010) found $\mathrm{HN}^{13} \mathrm{C}$ column densities of $\sim 2.6 \times$ $10^{12}-1.4 \times 10^{13} \mathrm{~cm}^{-2}$ towards IRDCs, quite similar to our values of $9.6 \times 10^{12}-1.5 \times 10^{13} \mathrm{~cm}^{-2}$. Vasyunina et al. $(2011)$ derived $x(\mathrm{HCN})$ and $x(\mathrm{HNC})$ values of $3.3 \times 10^{-10}-6.8 \times 10^{-9}$ (average $\left.2 \times 10^{-9}\right)$ and $2.4 \times 10^{-10}-6.3 \times 10^{-9}$ (average $\left.1.6 \times 10^{-9}\right)$, respectively. In general, these are comparable to our values, although the average HCN abundance we found is an order of magnitude higher than that derived by VLH11. The column densities and fractional abundances of HNC found by SJF12 are $5.1 \times 10^{13}-1.36 \times 10^{15} \mathrm{~cm}^{-2}$ and $5.1 \times 10^{-9}-1.85 \times 10^{-7}$. Their median values of $2.42 \times 10^{14} \mathrm{~cm}^{-2}$ and $3.73 \times 10^{-8}$ are both clearly higher than our values. Among the sample of SJF12, $N(\mathrm{HNC})$ was found to slightly increase with the clump evolution up to the active phase (when the clump shows an extended $4.5 \mu \mathrm{m}$ emission and hosts an embedded $24 \mu \mathrm{m}$ source), possibly as a result of accretion of the ambient material as suggested by the authors. However, the authors did not find evidence of increasing $x(\mathrm{HNC})$ as a function of source evolution. In contrast, we found higher $N(\mathrm{HNC})$ values towards IR-dark clumps on average than towards IR-bright clumps (the same holds for the median values also). However, the average HNC abundance (but not the median value) appears to be slightly higher in IR-bright clumps.

As shown in the top left panel of Fig. 2, there is a slight hint that $x(\mathrm{HCN})$ decreases when the $\mathrm{H}_{2}$ column density increases. Although the correlation coefficient is quite low $(r=-0.55)$, this could be related to the enhanced abundance of $\mathrm{HCO}^{+}$in more evolved (i.e. denser) sources ${ }^{8} ; \mathrm{HCO}^{+}$destroys $\mathrm{HCN}$ producing $\mathrm{H}_{2} \mathrm{CN}^{+}$ions (see above). The middle left panel in Fig. 2 shows

\footnotetext{
8 A higher $\mathrm{H}_{2}$ column density may not necessarily indicate a more advanced evolutionary stage. For example, Hoq et al. (2013) found no clear tendency for more evolved clumps to have a higher $\mathrm{H}_{2}$ column density (their Fig. 3).
}

that the $\mathrm{HNC}$ abundance increases when the $\mathrm{HCO}^{+}$abundance is enhanced. This can be understood as an increased production of $\mathrm{H}_{2} \mathrm{CN}^{+}$from $\mathrm{HCO}^{+}$. The dissociative recombination of $\mathrm{H}_{2} \mathrm{CN}^{+}$ then leads to the production of HNC (see above). The top right panel plot of Fig. 2 suggests that the HCN abundance increases when that of HNC increases. This is reminiscent of the positive correlation between the integrated intensities of $\mathrm{HCN}$ and HNC found by Liu et al. (2013; their Fig. 17). They also found a similar correlation between $\mathrm{HCN}$ and $\mathrm{HCO}^{+}$, in agreement with our relationship shown in the middle left panel of Fig. 2. Our positive $x\left(\mathrm{HCO}^{+}\right)-x(\mathrm{HNC})$ correlation supports the scenario where the HNC abundance increases as the clump evolves. However, if $x(\mathrm{HCN})$ also increases as our Fig. 2 (top right panel) suggests, the possible negative correlation we found between $N\left(\mathrm{H}_{2}\right)$ and $x(\mathrm{HCN})$ becomes questionable.

The values of the $\mathrm{HNC} / \mathrm{HCN}$ ratio found towards the clumps lie in the range of $0.01 \pm 0.002-6.38 \pm 3.05$, with the mean \pm std of $1.26 \pm 1.78$ (median is 0.47 ). The average value resembles the result by VLH11 who found that for their IRDCs the $\mathrm{HNC} / \mathrm{HCN}$ ratio is $\sim 1$. Liu et al. (2013) determined values in the range $0.71 \pm 0.11-2.26 \pm 0.44$ (average $1.47 \pm 0.50$ ) towards IRDCs, also comparable to our average value. In the dark cloud cores studied by Hirota et al. (1998), the HNC/HCN ratio was found to be $0.54 \pm 0.33-4.5 \pm 1.2(2.1 \pm 1.2$ on average), mostly comparable to our values within the errors. The above results are consistent with the near unity $\mathrm{HNC} / \mathrm{HCN}$ ratio theoretically expected in cold molecular clouds (Sarrasin et al. 2010). However, the abundance ratio between $\mathrm{HNC}$ and $\mathrm{HCN}$ is strongly dependent on the temperature. A good example is the Orion molecular cloud, where the $\mathrm{HNC} / \mathrm{HCN}$ ratio was found to strongly decrease (by more than an order of magnitude) when going from the colder quiescent parts of the cloud to the warm plateau and hot-core regions where the ratio is much smaller than unity (Goldsmith et al. 1986; cf. Schilke et al. 1992). Hirota et al. (1998) found that the $\mathrm{HNC} / \mathrm{HCN}$ ratio starts to rapidly decrease when the gas kinetic temperature rises above $24 \mathrm{~K}$, because of HNC conversion into HCN. The mean \pm std (median) of the $\mathrm{HNC} / \mathrm{HCN}$ ratio towards our IR-dark clumps is $0.34 \pm 0.34$ (0.24), while that for IR-bright clumps is $1.49 \pm 1.93(0.55)$. Because of the large scatter of these values, it is difficult to say how well (or poorly) they agree with the earlier studies of the temperature dependence of the $\mathrm{HNC} / \mathrm{HCN}$ ratio. Moreover, the clump temperatures should be determined in order to study this behaviour more quantitatively. Hoq et al. (2013) examined the integrated intensity ratios between $\mathrm{HNC}$ and $\mathrm{HCN}$, and found the median values of $0.9,0.8$, and 0.6 for quiescent IR-dark clumps, clumps containing YSOs, and clumps associated with $\mathrm{H}$ II regions/PDRs. This suggests a possible, although weak, evolutionary trend (see Fig. 4 in Hoq et al. 2013).

The column density ratio between $\mathrm{HNC}$ and $\mathrm{HCO}^{+}$is found to lie in the range $0.25 \pm 0.12-16.49 \pm 6.13$ with a mean \pm std of $4.29 \pm 3.94$ (median is 2.78 ) (the values for the additional velocity components are excluded here). Within the errors, this is in agreement with the gas-phase chemical models of cold dark clouds which suggest comparable abundances for these species (see Roberts et al. 2012 and references therein). Moreover, the average $\mathrm{HN}^{13} \mathrm{C}$ and $\mathrm{H}^{13} \mathrm{CO}^{+}$abundances, $1.2 \times 10^{-10}$ and $9.7 \times 10^{-11}$, are very similar. This could be indicative of weak ${ }^{13} \mathrm{C}$ fractionation effects. For IR-dark clumps, the mean \pm std and median values of the $\mathrm{HNC} / \mathrm{HCO}^{+}$ratio are $4.55 \pm 2.46$ and 4.36. For IR-bright sources the corresponding values are $4.19 \pm 4.47$ and 2.47. It is therefore possible that the $\mathrm{HNC} / \mathrm{HCO}^{+}$ ratio decreases slightly as the source evolves. This trend can be seen from the data in SJF12 when the clump evolves from 
the so-called intermediate state (either extended $4.5 \mu \mathrm{m}$ emission or an associated $24 \mu \mathrm{m}$ source, but not both) to the red state. However, the quiescent sources of SJF12 do not follow this trend.

\section{4. $\mathrm{C}_{2} \mathrm{H}$ (ethynyl)}

The origin of $\mathrm{C}_{2} \mathrm{H}$ in the PDR regions of the interstellar medium, i.e. at the boundary layers between ionised and molecular gas, is believed to be in the photodissociation of acetylene $\left(\mathrm{C}_{2} \mathrm{H}_{2}\right)$ : $\mathrm{C}_{2} \mathrm{H}_{2}+h v \rightarrow \mathrm{C}_{2} \mathrm{H}+\mathrm{H}$ (e.g. Fuente et al. 1993). The neutralneutral reaction $\mathrm{CH}_{2}+\mathrm{C} \rightarrow \mathrm{C}_{2} \mathrm{H}+\mathrm{H}$ can also produce $\mathrm{C}_{2} \mathrm{H}$, where the precursor carbon atom is formed through the photodissociation of CO (e.g. Turner et al. 2000). The dissociative recombination of the ions $\mathrm{CH}^{+}$(Rimmer et al. 2012), $\mathrm{C}_{2} \mathrm{H}_{2}^{+}$, and $\mathrm{C}_{2} \mathrm{H}_{3}^{+}$also yield ethynyl molecules in dense molecular clouds (Mul \& McGowan 1980). The $\mathrm{C}_{2} \mathrm{H}$ molecules can themselves be photodissociated back to form $\mathrm{C}_{2}$ and $\mathrm{C}_{2} \mathrm{H}^{+}$(Fuente et al. 1993). $\mathrm{C}_{2} \mathrm{H}$ is also destroyed through the reactions $\mathrm{C}_{2} \mathrm{H}+\mathrm{O} \rightarrow \mathrm{CO}+\mathrm{CH}$ and $\mathrm{C}_{2} \mathrm{H}+\mathrm{C}^{+} \rightarrow \mathrm{C}_{3}^{+}+\mathrm{H}$ (Watt et al. 1988).

The spatial distribution of the $\mathrm{C}_{2} \mathrm{H}$ emission is found to be quite extended (e.g. the clumps G1.87-SMM 1, G1.87-SMM 20, 23, 30, and G13.22-SMM 32). It is also worth noting that the emission is ridge-like around the IR-bubble pair N10/11 on the side of G13.22-SMM 4 and 5, and extended on the other side containing G13.22-SMM 6, 7, 10, and 11. In N10/11, and towards G13.22-SMM 32, we are probably probing the PDR parts of the sources, where the origin of $\mathrm{C}_{2} \mathrm{H}$ can be understood in terms of UV photodissociation. Only weak emission of $\mathrm{C}_{2} \mathrm{H}$ is seen towards G1.87-SMM 38, the G11.36 filament, and G13.22-SMM 23, 27, and 29. In the massive starforming clumps NGC $6334 \mathrm{E}$ and I, both associated with H II regions, the absence of $\mathrm{C}_{2} \mathrm{H}$ emission is suggested to be caused by the destruction of the molecules in such harsh environments (Walsh et al. 2010). Sanhueza et al. (2013) mapped the IRDC G028.23-00.19 in $\mathrm{C}_{2} \mathrm{H}$, and found the emission to come from the cold central part of the cloud, instead of from the outer layers. The authors suggested that $\mathrm{C}_{2} \mathrm{H}$ is tracing the dense and cold gas in their IRDC, which also seems to be the case in some of our clumps. Beuther et al. (2008) also found that $\mathrm{C}_{2} \mathrm{H}$ is prevalent during the early stages of star formation (and not only in PDRs). They suggested that $\mathrm{C}_{2} \mathrm{H}$ starts to decrease in abundance at the hot-core phase as a result of transformation to other species (e.g. $\mathrm{CO}$ from $\mathrm{O}$; see above). Only in the outer layers of the source, where UV photons produce elemental $\mathrm{C}$ from $\mathrm{CO}$ does the abundance of $\mathrm{C}_{2} \mathrm{H}$ can remain high. Furthermore, it was proposed by the authors that $\mathrm{C}_{2} \mathrm{H}$ could be a useful tracer of the initial conditions of high-mass star formation.

The $\mathrm{C}_{2} \mathrm{H}$ column densities we found, $7.8 \times 10^{13}-1.2 \times$ $10^{15} \mathrm{~cm}^{-2}\left(4.3 \times 10^{14} \mathrm{~cm}^{-2}\right.$ on average $)$, are quite similar to those derived by Sakai et al. (2008) towards their IRDC sources $\left(5.4 \times 10^{13}-2.9 \times 10^{14} \mathrm{~cm}^{-2}\right.$, the average being $\left.1.5 \times 10^{14} \mathrm{~cm}^{-2}\right)$. The fractional abundances we found are $1.8 \times 10^{-9}-2.4 \times 10^{-8}$ $\left(8.1 \times 10^{-9}\right.$ on average $)$. Similarly to us, VLH11 found abundances in the range $2.5 \times 10^{-9}-5.3 \times 10^{-8}\left(1.4 \times 10^{-8}\right.$ on average). Sanhueza et al. (2012) derived the values of $6.1 \times$ $10^{13}-7.75 \times 10^{14} \mathrm{~cm}^{-2}$ and $7.5 \times 10^{-9}-1.62 \times 10^{-7}$ for the column densities and abundances of $\mathrm{C}_{2} \mathrm{H}$. Their median column density of $2.41 \times 10^{14} \mathrm{~cm}^{-2}$ is similar to our value, but their median abundance of $3.72 \times 10^{-8}$ is almost six times higher. We found that both the mean and median values of the $\mathrm{C}_{2} \mathrm{H}$ abundance are higher towards IR-dark clumps than towards IR-bright clumps, and the lowest $\mathrm{C}_{2} \mathrm{H}$ abundance $(1.8 \times$ $10^{-9}$ ) is seen towards G13.22-SMM 29, the clump associated with IRAS 18117-1738 and probably (at least) in the hotcore phase of evolution. This is in accordance with the results by Beuther et al. (2008). On the other hand, SJF12 found no clear evolutionary trends for $N\left(\mathrm{C}_{2} \mathrm{H}\right)$ or $x\left(\mathrm{C}_{2} \mathrm{H}\right)$.

\subsection{HNCO (isocyanic acid)}

$\mathrm{HNCO}$ is a ubiquitous molecule in the interstellar medium. It was detected for the first time over $40 \mathrm{yr}$ ago in Sgr B2 by Snyder \& Buhl (1971). Since then, HNCO has been detected in, e.g. dark clouds (TMC-1; Brown 1981), low-mass protostellar cores (IRAS 16293-2422; van Dishoeck et al. 1995), hot cores/UC H II regions (e.g. G34.3+0.15; MacDonald et al. 1996), translucent clouds (Turner et al. 1999), and outflows emanating from massive YSOs (IRAS 17233-3606; Leurini et al. 2011). Zinchenko et al. (2000) searched for HNCO emission towards a sample of 81 dense molecular cloud cores, and detected it in $57(70 \%)$ of them. Jackson et al. (1984) proposed that HNCO is tracing the densest $\left(\gtrsim 10^{6} \mathrm{~cm}^{-3}\right)$ parts of molecular clouds. Other authors have suggested that $\mathrm{HNCO}$ could be tracing shocks because it is found to correlate with $\mathrm{SiO}$ emission (e.g. Zinchenko et al. 2000; Minh \& Irvine 2006; Rodríguez-Fernández et al. 2010). Association of HNCO clumps with embedded YSOs was recently suggested by the Purple Mountain Observatory $13.7 \mathrm{~m}$ telescope observations by Li et al. (2013).

Gas-phase reactions that could be responsible for the formation of $\mathrm{HNCO}$ include the dissociative recombinations of $\mathrm{H}_{2} \mathrm{NCO}^{+}$(Iglesias 1977), $\mathrm{H}_{3} \mathrm{NCO}^{+}, \mathrm{CNH}_{3} \mathrm{O}^{+}$, and $\mathrm{CNH}_{2} \mathrm{O}^{+}$, and the neutral-neutral reaction between $\mathrm{NCO}$ and $\mathrm{H}_{2}$ (Turner et al. 1999). The compound HNCO is dominantly destroyed by reactions with $\mathrm{H}_{3}^{+}$and $\mathrm{He}^{+}$(Turner et al. 1999); it could also form through grain-surface chemistry, namely hydrogenation of accreted OCN (Garrod et al. 2008). In grain ices containing $\mathrm{H}_{2} \mathrm{O}$ and $\mathrm{NH}_{3}$, the $\mathrm{HNCO}$ molecules can react with these species to form $\mathrm{OCN}^{-}$anions (e.g. van Broekhuizen et al. 2004; Table 2 therein). More recently, Tideswell et al. (2010) found that gasphase reactions, even at hot-core temperatures, are unlikely to be able to produce the observed HNCO abundances. Instead, grain-surface pathways are required, although direct evaporation from the icy grain mantles is not sufficent itself. More complex molecules formed from $\mathrm{HNCO}$ on grain surfaces are expected to be evaporated into the gas phase; their dissociation can then lead to the formation of HNCO (Tideswell et al. 2010).

The $\operatorname{HNCO}\left(4_{0,4}-3_{0,3}\right)$ emission is found to be extended in many of the clumps (G1.87-SMM 1, 12, 14, 16, 20, 23 , 30). Particularly in G1.87-SMM 14, the HNCO emission peaks at the position of the strongest dust emission as traced by LABOCA (Fig. B.3). On the other hand, towards G1.87-SMM 20, 23, and 30, where the HNCO emission is also extended, the emission peak is not coincident with any of the submm peaks (Fig. B.5). Instead, the $\mathrm{HNCO}$ emission has its maximum at the position of the $\mathrm{SiO}, \mathrm{HNC}, \mathrm{HC}_{3} \mathrm{~N}, \mathrm{CH}_{3} \mathrm{CN}$, and $\mathrm{N}_{2} \mathrm{H}^{+}$emission maxima. We note that there is no Spitzer $24 \mu \mathrm{m}$ source within the Mopra beam in this molecular-line emission peak. Towards G1.87-SMM 1, the HNCO emission morphology is quite similar to that of $\mathrm{HC}_{3} \mathrm{~N}$ (Fig. B.1). Towards the clumps G13.22-SMM 23, 27, and 32 the HNCO emission is very weak (Figs. B.12 and B.14).

Based on the $4_{0,4}-3_{0,3}$ transition, the HNCO column densities and fractional abundances are determined to be $5.4 \times$ $10^{12}-7.9 \times 10^{14} \mathrm{~cm}^{-2}\left(2.2 \times 10^{14} \mathrm{~cm}^{-2}\right.$ on average $)$ and $1.3 \times$ $10^{-10}-1.9 \times 10^{-8}\left(6.6 \times 10^{-9}\right.$ on average $)$. The above values should be taken as lower limits only because of the assumption made for $T_{\text {ex }}$ (Sect. 3.3). Vasyunina et al. (2011) derived HNCO 
abundances of $2.2 \times 10^{-10}-3.7 \times 10^{-9}$. Their average value, $1.2 \times 10^{-9}$, is 5.5 times lower than ours. Sanhueza et al. (2012) found HNCO column densities in the range $\sim 9.8 \times 10^{12}-9.9 \times$ $10^{13} \mathrm{~cm}^{-2}$, with the median value of $3.36 \times 10^{13} \mathrm{~cm}^{-2}$, i.e. about four times lower than our value $\left(1.4 \times 10^{14} \mathrm{~cm}^{-2}\right)$. The authors found that the column density increases when the source evolves. Our median HNCO column densities agree with this trend, as do the mean and median fractional abundances. It is unclear whether our high $N(\mathrm{HNCO})$ values could point towards more evolved sources on average compared to those studied by $\mathrm{SJF} 12$, because the $\mathrm{HCO}^{+}$data suggest the opposite (Sect. 4.1). The fractional HNCO abundances found by SJF12 are $\sim 2.7 \times 10^{-10}-7.6 \times 10^{-9}$. Their median value, $2.8 \times 10^{-9}$, is only $\sim 1.8$ times lower than the value $5 \times 10^{-9}$ we found. In most of the clumps studied by SJF12, the HNCO line profiles showed no signatures of shocks. In contrast, we found that eleven of our clumps (G1.87-SMM 1, 8, 10, 14, 15, 21, 24, 27, 28, 31, and 38) show HNCO wing-emission indicative of outflows (and therefore shocks).

\section{6. $\mathrm{HC}_{3} \mathrm{~N}$ and $\mathrm{HC}^{13} \mathrm{CCN}$ (cyanoacetylene)}

Cyanopolyynes are organic chemical species that contain a chain of at least one C-C triple bond, alternating with single bonds and ending with a cyanide $(\mathrm{CN})$ group. The most simple example of cyanopolyynes is the cyanoacetylene $\mathrm{HC}_{3} \mathrm{~N}$. Its first interstellar detection was made towards Sgr B2 (Turner 1971). This molecule can trace both the cold molecular clouds (see below) and hot cores where it is formed through the gas-phase reaction $\mathrm{C}_{2} \mathrm{H}_{2}+\mathrm{CN} \rightarrow \mathrm{HC}_{3} \mathrm{~N}+\mathrm{H}$ after $\mathrm{C}_{2} \mathrm{H}_{2}$ is released from the grain mantles after heating (e.g. Chapman et al. 2009).

The $\mathrm{HC}_{3} \mathrm{~N}$ emission is found to be extended towards many of the observed fields. G1.87-SMM 1 and G1.87-SMM 28 are examples where the line emission peaks towards the dust emission peak. Similarly, Walsh et al. (2010) found that the $\mathrm{HC}_{3} \mathrm{~N}$ emission closely resembles that of the dust continuum emission in NGC 6334, and suggested that $\mathrm{HC}_{3} \mathrm{~N}$ is a good tracer of quiescent dense gas (see also Pratap et al. 1997 for the $\mathrm{HC}_{3} \mathrm{~N}$ emission along the quiescent TMC- 1 ridge). The sources G1.87-SMM 20, 23, and 30 are associated with a few parsecscale $\mathrm{HC}_{3} \mathrm{~N}$ clump, but the line emission peak is not coincident with any of the submm peaks. Instead, the emission morphology resembles those of $\mathrm{SiO}, \mathrm{HNCO}, \mathrm{HNC}$, and $\mathrm{N}_{2} \mathrm{H}^{+}$. In addition, the $\mathrm{CH}_{3} \mathrm{CN}$ emission towards the field peaks at the $\mathrm{HC}_{3} \mathrm{~N}$ peak. There is also a small $\mathrm{HC}_{3} \mathrm{~N}$ clump in G1.87-SMM 38, and its maximum is coincident with the dust peak. The clump G13.22-SMM 5, belonging to the N10/11 bubble system, is associated with a small $\mathrm{HC}_{3} \mathrm{~N}$ clump. On the other hand, towards G1.87-SMM 8, 10, 14-17, 21, 24, and G13.22-SMM 23, 27 the $\mathrm{HC}_{3} \mathrm{~N}$ emission is very weak or absent. The $\mathrm{HC}_{3} \mathrm{~N}$ line wings detected towards some of our clumps suggest the shockorigin for the emission (e.g. the case of the IR-dark clump G1.87-SMM 27).

The $\mathrm{HC}_{3} \mathrm{~N}$ column densities and abundances we derive are $7 \times 10^{12}-7.9 \times 10^{14} \mathrm{~cm}^{-2}$ and $1 \times 10^{-10}-2.7 \times 10^{-8}$. The mean values are $1.6 \times 10^{14} \mathrm{~cm}^{-2}$ and $5 \times 10^{-9}$. As we did in the case of the linear $\mathrm{SiO}$ molecule, we assumed that $T_{\mathrm{ex}}=E_{\mathrm{u}} / k_{\mathrm{B}}$ (Sect. 3.3), so the above values should be interpreted as lower limits. Sakai et al. (2008) determined $N\left(\mathrm{HC}_{3} \mathrm{~N}\right)$ values in the range $<2.2 \times 10^{12}-5.4 \times 10^{13} \mathrm{~cm}^{-2}$ for their sources. Our mean column density exceeds the highest value found by Sakai et al. (2008) by a factor of three. Vasyunina et al. (2011) derived $\mathrm{HC}_{3} \mathrm{~N}$ abundances of $1 \times 10^{-10}-1.5 \times 10^{-9}$ with an average value of $5.4 \times 10^{-10}$. Our average value is almost an order of magnitude higher. The $N\left(\mathrm{HC}_{3} \mathrm{~N}\right)$ values derived by SFJ12 are $1.4 \times 10^{12}-3.33 \times 10^{13} \mathrm{~cm}^{-2}$ (median $4.77 \times 10^{12} \mathrm{~cm}^{-2}$ ). Their median $N\left(\mathrm{HC}_{3} \mathrm{~N}\right)$ is about six times lower than ours, but the median abundance for their sample, $4.23 \times 10^{-10}$, is comparable to our value of $6.1 \times 10^{-10}$.

In agreement with the chemical model by Nomura \& Millar (2004), SJF12 found that $N\left(\mathrm{HC}_{3} \mathrm{~N}\right)$ increases as a function of clump evolutionary stage, but their median abundances did not show such a trend. Among the quiescent clumps they studied, $\mathrm{HC}_{3} \mathrm{~N}$ was detected in only one source. The trend between $N\left(\mathrm{HC}_{3} \mathrm{~N}\right)$ and the evolutionary stage found by SJF12 is not seen among our sources. On the other hand, we found that the average $\mathrm{HC}_{3} \mathrm{~N}$ abundance is very similar between the IR-dark and -bright clumps $\left(5.1 \times 10^{-9}\right.$ and $\left.4.7 \times 10^{-9}\right)$, while the median value is about 1.4 times higher towards the latter sources.

The $\mathrm{HC}_{3} \mathrm{~N} / \mathrm{HCN}$ ratios are found to lie in the range $0.02 \pm$ $0.01-0.24 \pm 0.06$, where the mean \pm std is $0.10 \pm 0.09$ (median is 0.07). For comparison, Dickens et al. (2000) found that the $\mathrm{HC}_{3} \mathrm{~N} / \mathrm{HCN}$ ratio is about $0.05-0.07$ in the $\mathrm{L} 134 \mathrm{~N}$ (L183) prestellar core. In comet Hale-Bopp, the above ratio was found to be $8.2 \%$ (Bockelée-Morvan et al. 2000). More recently, Chapillon et al. (2012), who made the first detection of $\mathrm{HC}_{3} \mathrm{~N}$ in protoplanetary disks, derived the $\mathrm{HC}_{3} \mathrm{~N} / \mathrm{HCN}$ ratios of $\leq 0.05$, 0.075, and 0.55 towards DM Tau, LkCa 15, and MWC 480, respectively. Interestingly, the abundance ratio between $\mathrm{HC}_{3} \mathrm{~N}$ and HCN appears to be quite similar in IRDC clumps, low-mass prestellar cores, disks around T Tauri stars, and comets 9 .

The ${ }^{13} \mathrm{C}$ isotopologue of $\mathrm{HC}_{3} \mathrm{~N}, \mathrm{HC}^{13} \mathrm{CCN}$, forms via the isotope exchange reaction ${ }^{13} \mathrm{C}^{+}+\mathrm{HC}_{3} \mathrm{~N} \rightarrow \mathrm{C}^{+}+\mathrm{HC}^{13} \mathrm{CCN}$ (Takano et al. 1998). No $\mathrm{HC}^{13} \mathrm{CCN}$ emission was detected in any of our sources. The $E_{\mathrm{u}} / k_{\mathrm{B}}$ value of the observed $\mathrm{HC}^{13} \mathrm{CCN}$ transition is very similar to that of $\mathrm{HC}_{3} \mathrm{~N}(\sim 24 \mathrm{~K})$, so the nondetection is not likely to be caused by excitation conditions. The probable reasons are the relative rareness of $\mathrm{HC}^{13} \mathrm{CCN}$, and the limited $\mathrm{S} / \mathrm{N}$ ratio of mapping observations. A positive detection is expected with a longer integration time/single-pointing observation, at least towards the strongest $\mathrm{HC}_{3} \mathrm{~N}$ sources. The compound $\mathrm{HC}^{13} \mathrm{CCN}$ was first detected in Sgr B2 by Gardner \& Winnewisser (1975), and Gibb et al. (2000) make a detection towards the hot core G327.3-0.6.

\section{7. ${ }^{13} \mathrm{CS}$ and ${ }^{13} \mathrm{C}^{34} \mathrm{~S}$ (carbon monosulfide)}

Only traces of ${ }^{13} \mathrm{CS}(2-1)$ emission are seen towards the clump G1.87-SMM 1 (Fig. B.1), and towards the field containing G1.87-SMM 27, 28, and 31 (Fig. B.6). The isotopologue ${ }^{13} \mathrm{C}^{34} \mathrm{~S}$ is not detected at all. Beuther \& Henning (2009) found only weak ${ }^{13} \mathrm{CS}(2-1)$ emission towards the IRDCs $19175-4$ and -5 , and derived very high CS depletion factors of $\sim 100$. Although CS can suffer from depletion onto dust grain surfaces in cold sources, its non-detection in the present study is probably caused by a limited $\mathrm{S} / \mathrm{N}$ ratio. The authors VLH11 detected only very weak ${ }^{13} \mathrm{CS}(2-1)$ emission in only three sources of their IRDC sample, with an average $x\left({ }^{13} \mathrm{CS}\right)$ of $3 \times 10^{-10}$.

\section{8. $\mathrm{CH}_{3} \mathrm{CN}$ (methyl cyanide)}

Methyl cyanide is a good tracer of warm/hot and dense parts of molecular clouds (e.g. Araya et al. 2005; Purcell et al. 2006).

9 The high $\mathrm{HC}_{3} \mathrm{~N} / \mathrm{HCN}$ ratio in the MWC 480 disk is an exception due to the warmer dust present, which enhances the diffusion of radicals on grain surfaces and leads to a higher abundance of solid $\mathrm{HC}_{3} \mathrm{~N}$ (Chapillon et al. 2012). 
The formation of $\mathrm{CH}_{3} \mathrm{CN}$ probably takes place on the grain surfaces during the earliest stages of YSO evolution. The most efficient route for this is the reaction between $\mathrm{CH}_{3}$ and $\mathrm{CN}$ (e.g. Garrod et al. 2008). Later, when the central star starts to heat its surrounding medium, $\mathrm{CH}_{3} \mathrm{CN}$ evaporates into the gas phase. In the gas phase, $\mathrm{CH}_{3} \mathrm{CN}$ can form when $\mathrm{CH}_{3}^{+}$and $\mathrm{HCN}$ first form $\mathrm{H}_{4} \mathrm{C}_{2} \mathrm{~N}^{+}$ions through radiative association, and which then dissociatively recombine with electrons to form $\mathrm{CH}_{3} \mathrm{CN}$ molecules and $\mathrm{H}$-atoms (e.g. Mackay 1999). Shocks associated with YSO outflows can also be responsible for the production of $\mathrm{CH}_{3} \mathrm{CN}$ (Codella et al. 2009). The $\mathrm{CH}_{3} \mathrm{CN}$ molecules can be photodissociated into $\mathrm{CH}_{3}$ and $\mathrm{CN}$ molecules (Mackay 1999).

Inspection of the $\mathrm{CH}_{3} \mathrm{CN}$ maps shows that the emission is quite weak in most cases (e.g. G1.87-SMM 12, 14, 16, 27, 28, and 31). Towards G1.87-SMM 1, the $\mathrm{CH}_{3} \mathrm{CN}$ emission is extended (although weak) around the submm peak (Fig. B.1). Interestingly, the clump appears to be IR-dark, so perhaps the emission has its origin in shocks. Near the submm peaks G1.87-SMM 20, 23, and 30, there is a pc-scale $\mathrm{CH}_{3} \mathrm{CN}$ clump, with the emission peak being coincident with the $\mathrm{SiO}, \mathrm{HNCO}$, $\mathrm{HNC}, \mathrm{HC}_{3} \mathrm{~N}$, and $\mathrm{N}_{2} \mathrm{H}^{+}$maxima (Fig. B.5). Here too, the shockorigin of $\mathrm{CH}_{3} \mathrm{CN}$ is supported by the coincidence with the $\mathrm{SiO}$ and $\mathrm{HNCO}$ emission peaks.

The $\mathrm{CH}_{3} \mathrm{CN}$ column densities and fractional abundances could be derived only towards three positions (where the extracted spectra showed visible lines). These are $5.5 \times 10^{11} \mathrm{~cm}^{-2}$ and $\sim 10^{-11}$ on average and are only lower limits because we assumed that $T_{\mathrm{ex}}=2 E_{\mathrm{u}} / 3 k_{\mathrm{B}}$ (Sect. 3.3). Beuther \& Sridharan (2007) found average values higher by one order of magnitude for both the column density and abundance towards their IRDC sources. However, they used single-pointing observations with the IRAM $30 \mathrm{~m}$ telescope, while we have used OTF-data with lower $\mathrm{S} / \mathrm{N}$ ratios. It is also possible that our sources are in an earlier stage of evolution and therefore show only weak $\mathrm{CH}_{3} \mathrm{CN}$ emission. For example, Nomura \& Millar (2004) modelled the chemistry of the hot core $\mathrm{G} 34.3+0.15$, and found that the $\mathrm{CH}_{3} \mathrm{CN}$ column density is at the level we have derived when the source's age is only $\lesssim 10^{3} \mathrm{yr}$. Moreover, Vasyunina et al. (2011) detected no $\mathrm{CH}_{3} \mathrm{CN}$ emission towards their IRDCs, and SJF12 found $\mathrm{CH}_{3} \mathrm{CN}$ emission in only one active (extended $4.5 \mu \mathrm{m}$ emission in addition to a YSO seen at $24 \mu \mathrm{m}$ ) clump (G034.43 MM1) of their large sample of IRDC sources. The hot cores studied by Bisschop et al. (2007) show $\mathrm{CH}_{3} \mathrm{CN}$ abundances of $1.5 \times 10^{-8}-1.5 \times 10^{-7}$, much higher than seen towards IRDCs.

\section{9. $H 41 \alpha$}

The H41 $\alpha$ recombination line emission is not detected in this study. It is known that some of our clumps do contain H II regions, and in these partially or fully ionised gas regions protons can capture electrons. Therefore, these H II regions are expected to emit recombination lines. The non-detection towards the clumps with $\mathrm{HII}$ regions is probably caused by our low $\mathrm{S} / \mathrm{N}$ ratio (similarly to $\mathrm{HC}^{13} \mathrm{CCN}$ and ${ }^{13} \mathrm{CS}$ ). For example, Araya et al. (2005), who used single-pointing $15 \mathrm{~m}$ SEST (SwedishESO Submillimetre Telescope) telescope observations, detected $\mathrm{H} 41 \alpha$ line emission in nine sites of high-mass star formation. More recently, Klaassen et al. (2013) detected H41 $\alpha$ towards the high-mass star-forming region K3-50A using the CARMA (Combined Array for Research in Millimetre-wave Astronomy) interferometer, and suggested that the line is tracing the outflow entrained by photoionised gas.

\subsection{0. $\mathrm{N}_{2} \mathrm{H}^{+}$(diazenylium)}

Owing to its resistance of depletion at low temperatures and high densities, $\mathrm{N}_{2} \mathrm{H}^{+}$is an excellent tracer of cold and dense molecular clouds (e.g. Caselli et al. 2002). Diazenylium molecules are primarily formed through the gas-phase reaction $\mathrm{H}_{3}^{+}+\mathrm{N}_{2} \rightarrow \mathrm{N}_{2} \mathrm{H}^{+}+\mathrm{H}_{2}$. When there are $\mathrm{CO}$ molecules in the gas phase, they can destroy $\mathrm{N}_{2} \mathrm{H}^{+}$producing $\mathrm{HCO}^{+}\left(\mathrm{N}_{2} \mathrm{H}^{+}+\mathrm{CO} \rightarrow\right.$ $\mathrm{HCO}^{+}+\mathrm{N}_{2}$ ). When $\mathrm{CO}$ has depleted via freeze-out onto dust grains, $\mathrm{N}_{2} \mathrm{H}^{+}$is mainly destroyed in the electron recombination $\left(\mathrm{N}_{2} \mathrm{H}^{+}+\mathrm{e}^{-} \rightarrow \mathrm{N}_{2}+\mathrm{H}\right.$ or $\left.\mathrm{NH}+\mathrm{N}\right)$.

The $\mathrm{N}_{2} \mathrm{H}^{+}$emission is found to be extended towards many of our fields (e.g. the fields covering G1.87-SMM 1; G1.87-SMM 12, 14, 16; G1.87-SMM 20, 23, 30; G1.87-SMM 27, 28, 31; G1.87-SMM 38; G13.22-SMM 23, 27; G13.22-SMM 32). The $\mathrm{N}_{2} \mathrm{H}^{+}$emission also traces well the G11.36 filament as seen by LABOCA at $870 \mu \mathrm{m}$ and in absorption by Spitzer at $8 \mu \mathrm{m}$ (Fig. B.9). Very strong and extended $\mathrm{N}_{2} \mathrm{H}^{+}$emission is seen around the N10/11 double bubble, and the emission peaks are coincident with the submm peaks G13.22-SMM 5 and 7 (Figs. B.10 and B.11). In G1.87-SMM 38 the line emission also peaks at the dust peak (Fig. B.7). Interestingly, towards G1.87-SMM 20, 23, 30 , the $\mathrm{N}_{2} \mathrm{H}^{+}$emission peaks in between the submm dust emission maxima, where the line emission from several other species has its maximum, as discussed earlier. In addition, in G13.22-SMM 23, 27, and 32 the $\mathrm{N}_{2} \mathrm{H}^{+}$emission peaks are offset from the submm peaks by $17^{\prime \prime}, 15^{\prime \prime} .9$, and $30{ }^{\prime} .5$, respectively. The pc-scale $\mathrm{N}_{2} \mathrm{H}^{+}$clump in G2.11-SMM 5 has its maximum at about $11^{\prime \prime} .6(0.42 \mathrm{pc})$ in projection from the strongest dust emission. Ragan et al. (2006) and Liu et al. (2013), who mapped IRDCs in the $J=1-0$ line of $\mathrm{N}_{2} \mathrm{H}^{+}$, also found good correspondence between the line emission and $8 \mu \mathrm{m}$ absorption.

We found $\mathrm{N}_{2} \mathrm{H}^{+}$column densities in the range of $6.6 \times$ $10^{12}-5.4 \times 10^{14} \mathrm{~cm}^{-2}$, and abundances of $2.8 \times 10^{-10}-9.8 \times$ $10^{-9}$. The average values are $5.4 \times 10^{13} \mathrm{~cm}^{-2}$ and $1.6 \times 10^{-9}$, respectively. Ragan et al. (2006) derived comparable abundances of $5.7 \times 10^{-11}-2 \times 10^{-9}$ for their sample of IRDCs. The column density range found by Sakai et al. (2008) for their IRDCs, $\sim 4.3 \times 10^{12}-1.3 \times 10^{14} \mathrm{~cm}^{-2}$, is also very similar to our range of values. Vasyunina et al. (2011) derived fractional $\mathrm{N}_{2} \mathrm{H}^{+}$abundances of $1.9 \times 10^{-10}-8.5 \times 10^{-9}$ with an average of $2.8 \times 10^{-9}$, remarkably similar to our values. More recently, SJF12 derived column densities of $3.6 \times 10^{12}-1.37 \times 10^{14} \mathrm{~cm}^{-2}(\mathrm{me}-$ dian is $\left.1.6 \times 10^{13} \mathrm{~cm}^{-2}\right)$ and abundances in the range $1.9 \times$ $10^{-10}-1.68 \times 10^{-8}$ (the median being $2.4 \times 10^{-9}$ ), again similar to our results. They also found that both the values of $N\left(\mathrm{~N}_{2} \mathrm{H}^{+}\right)$ and $x\left(\mathrm{~N}_{2} \mathrm{H}^{+}\right)$increase as the clump evolves from the quiescent (IR-dark) to the star-forming stage. Similarly, Hoq et al. (2013) found that $x\left(\mathrm{~N}_{2} \mathrm{H}^{+}\right)$rises as a function of evolutionary stage (their Fig. 5). The reason for this correlation is somewhat unclear because of the destroying effect of $\mathrm{CO}$ (see above), and in contrast, our clumps show the opposite trend. Sanhueza et al. (2012) suggested that the rate coefficients of the relevant chemical reactions might be inaccurate, and/or the large beam size of Mopra observations is seeing the cold $\mathrm{N}_{2} \mathrm{H}^{+}$gas around the warmer central YSOs, thus leading to their observed correlation. On the other hand, higher temperature could lead to an enhanced evaporation of $\mathrm{N}_{2}$ from the dust grains, thus allowing $\mathrm{N}_{2} \mathrm{H}^{+}$to increase in abundance (Chen et al. 2013). Finally, Liu et al. (2013) derived $\mathrm{N}_{2} \mathrm{H}^{+}$column densities of $\sim 3.3 \times 10^{12}-2.7 \times 10^{13} \mathrm{~cm}^{-2}$ with the mean value being $\sim 9.4 \times 10^{12} \mathrm{~cm}^{-2}$, a factor of about six lower than ours. 
The $\mathrm{N}_{2} \mathrm{H}^{+} / \mathrm{HCO}^{+}$ratios we derived lie in the range $0.14 \pm$ $0.08-18.57 \pm 3.41$, i.e. the ratio varies a lot. The mean \pm std is $4.48 \pm 4.26$, and the median value is 3.36 . Separately for IR-dark and -bright clumps, these values are $6.59 \pm 5.82$ and 3.53, and $8.49 \pm 22.61$ and 2.54, respectively. Because of the large dispersion in these values, there is no clear correlation between the clump evolutionary stage and the value of the $\mathrm{N}_{2} \mathrm{H}^{+} / \mathrm{HCO}^{+}$ ratio among our source sample. However, one would expect to observe a higher ratio of $\mathrm{N}_{2} \mathrm{H}^{+} / \mathrm{HCO}^{+}$towards clumps in the earliest stages of evolution, which is actually mildly suggested by our median values. This is because $\mathrm{CO}$ is expected to be depleted in starless clumps, so that $\mathrm{HCO}^{+}$cannot form via the reaction between $\mathrm{H}_{3}^{+}$and $\mathrm{CO}$. When the source evolves, it gets warmer and $\mathrm{CO}$ should be evaporated from the dust grains when the dust temperature exceeds about $\sim 20 \mathrm{~K}$ (see Tobin et al. 2013). Two effects can then follow: the $\mathrm{CO}$ molecules can start to form $\mathrm{HCO}^{+}$, and destroy $\mathrm{N}_{2} \mathrm{H}^{+}$. As a consequence, the $\mathrm{N}_{2} \mathrm{H}^{+} / \mathrm{HCO}^{+}$ratio should decrease. Sanhueza et al. (2012) found that the $\mathrm{N}_{2} \mathrm{H}^{+} / \mathrm{HCO}^{+}$abundance ratio decreases from intermediate to active and red clumps, and therefore acts as a chemical clock in accordance with the scenario described above. However, the quiescent clumps studied by SJF12 do not follow this trend. As an explanation for this, the authors suggested, for example, that some of them could contain YSOs not detected by Spitzer, or that CO has not had enough time to deplete from the gas phase. During the starless phase of evolution, however, the $\mathrm{N}_{2} \mathrm{H}^{+} / \mathrm{HCO}^{+}$ratio is expected to increase (CO becomes increasingly depleted and cannot destroy $\mathrm{N}_{2} \mathrm{H}^{+}$). Hoq et al. (2013) also inspected whether there is an evolutionary trend in the $\mathrm{N}_{2} \mathrm{H}^{+} / \mathrm{HCO}^{+}$abundance ratio, and the median value appeared to be slightly higher in more evolved clumps, although, as the authors stated, the result is not statistically significant (their Fig. 6). Hoq et al. (2013) employed the chemical evolution model by Vasyunina et al. (2012) to further investigate the behaviour of the $\mathrm{N}_{2} \mathrm{H}^{+} / \mathrm{HCO}^{+}$ratio. As shown in their Fig. 10, the abundance ratio appears to increase as a function of time until the peak value is reached at $\sim 10^{4} \mathrm{yr}$.

We also derived the $\mathrm{N}_{2} \mathrm{H}^{+} / \mathrm{HNC}$ ratios, ranging from $0.08 \pm$ 0.04 to $4.32 \pm 7.64$. The mean \pm std is $1.26 \pm 1.07$, and the median value is 0.87 . For IR-dark clumps these values are $1.20 \pm 1.17$ and 0.72 , while for IR-bright clumps they are $1.26 \pm 1.05$ and 1.17 . The median $\mathrm{N}_{2} \mathrm{H}^{+} / \mathrm{HNC}$ ratio found by SJF12 for all their clumps (0.07) is comparable to the lowest values we found, while our median is over 12 times higher. For the IRDC sample examined by Liu et al. (2013), the ratio ranges from $\sim 0.54-3.86$ with the mean \pm std of $1.20 \pm 0.79$ and median of 1.07 (computed from the values in their Table 4). These are comparable to our values. Sanhueza et al. (2012) suggested that there is a trend of increasing $\mathrm{N}_{2} \mathrm{H}^{+} / \mathrm{HNC}$ ratio with the clump evolution, but the change in their median value is very small, only from 0.06 for quiescent clumps to 0.08 for active and red clumps. Our clumps show a similar, except stronger, trend in median values (the average ratios also suggest this trend). Sanhueza et al. (2012) proposed that HNC could be preferentially formed during the cold phase, while their median $\mathrm{N}_{2} \mathrm{H}^{+}$abundance appeared to increase when the source evolves. We found that there is a positive $\mathrm{N}_{2} \mathrm{H}^{+}-\mathrm{HCN}$ abundance correlation, as shown in the middle right panel of Fig. 2. A weaker, although possible positive correlation, is also found between $\mathrm{N}_{2} \mathrm{H}^{+}$and $\mathrm{HNC}$ (Fig. 2, bottom panel). According to the Liu et al. (2013) results, and our results agree, the $\mathrm{N}_{2} \mathrm{H}^{+} / \mathrm{HNC}$ ratio is near unity on average, although the std is very large. If the increasing trend in $x$ (HNC) as a function of $x\left(\mathrm{~N}_{2} \mathrm{H}^{+}\right)$is real, the abundance ratio is not expected to change significantly, which agrees with the results in SJF12 and of the present study.

\section{Summary and conclusions}

Altogether, 14 subfields from the LABOCA $870 \mu \mathrm{m}$ survey of IRDCs by Miettinen (2012b) are covered by the MALT90 molecular-line survey. These IRDC fields contain 35 clumps in total, ranging from quiescent (IR-dark) sources to clumps associated with $\mathrm{H}$ II regions. In the present study, the MALT90 observations were used to investigate the chemical properties of the clumps. Our main results and conclusions are summarised as follows:

1. Of the 16 transitions at $\lambda \approx 3 \mathrm{~mm}$ included in the MALT90 survey, all except five $\left[\mathrm{HNCO}\left(4_{1,3}-3_{1,2}\right), \mathrm{HC}^{13} \mathrm{CCN}(10-9)\right.$, ${ }^{13} \mathrm{C}^{34} \mathrm{~S}(2-1), \mathrm{H} 41 \alpha$, and $\left.{ }^{13} \mathrm{CS}(2-1)\right]$ are detected towards our sources.

2. The $\mathrm{HCO}^{+}(1-0)$ emission is extended in many of the clumps, resembling the MALT90 mapping results of IRDCs by Liu et al. (2013). The fractional $\mathrm{HCO}^{+}$abundances appear to be lower than in the IRDC clumps studied by Vasyunina et al. (2011) and Sanhueza et al. (2012), who also used the Mopra telescope observations. We found that the average $\mathrm{HCO}^{+}$abundance increases when the clump evolves (the median $\mathrm{HCO}^{+}$abundance is very similar between the IR-dark and -bright clumps), resembling the trend discovered by Sanhueza et al. (2012) and Hoq et al. (2013). The $\mathrm{H}^{13} \mathrm{CO}^{+}(1-0)$ emission is generally weak in our clumps, and its average abundance is a factor of 3.5 lower than in the sources of Vasyunina et al. (2011).

3. Extendend or clump-like $\mathrm{SiO}(2-1)$ emission is seen towards several clumps. In three cases, the maximum of the integrated intensity of $\mathrm{SiO}$ is coincident with the LABOCA $870 \mu \mathrm{m}$ peak position. As supported by the observed line wings, $\mathrm{SiO}$ emission is probably caused by outflow activity which generates shocks releasing $\mathrm{SiO}$ into the gas phase. However, some of the widespread $\mathrm{SiO}$ emission could result from shocks associated with cloud formation as suggested in the IRDC G035.39-00.33 (Jiménez-Serra et al. 2010). The $\mathrm{SiO}$ abundances derived for our clumps are mostly comparable to those seen in other IRDCs (Vasyunina et al. 2011; Sanhueza et al. 2012). No trend of decreasing SiO abundance with the clump evolution is seen, as suggested by some earlier studies of massive clumps (Miettinen et al. 2006; Sakai et al. 2010). Instead, our data suggest the opposite trend, as seen in the data by Sanhueza et al. (2012).

4. The $J=1-0$ line emission of HCN and HNC is generally found to be spatially extended. The fractional abundances of these species are mostly comparable to those found by Vasyunina et al. (2011), but Sanhueza et al. (2012) derived clearly higher $\mathrm{HNC}$ abundances. We found no evidence of increasing HNC column density as the source evolves as Sanhueza et al. (2012) did. However, our average HNC abundance (but not the median value) appears to be slightly higher in more evolved sources. This is perhaps related to the accumulation of gas from the surrounding mass reservoir (Sanhueza et al. 2012). We found a hint that the HCN abundance decreases when the column density of molecular hydrogen increases. This might be related to the enhanced $\mathrm{HCO}^{+}$abundance in more evolved sources, where the species can destroy $\mathrm{HCN}$ molecules to form $\mathrm{H}_{2} \mathrm{CN}^{+}$and $\mathrm{CO}$. The HNC abundance is found to increase as a function of the $\mathrm{HCO}^{+}$abundance. This is probably related to 
the increased production of $\mathrm{H}_{2} \mathrm{CN}^{+}$, a molecular ion that forms $\mathrm{HNC}$ in the dissociative recombination with an electron. The $\mathrm{HCN}$ fractional abundance appears to increase when that of HNC increases, in agreement with the result by Liu et al. (2013). In this case, however, the decrease in $x(\mathrm{HCN})$ as a function of time is questionable if HNC gets more abundant as the clump evolves. The $\mathrm{HNC} / \mathrm{HCN}$ ratio is found to lie in the range $\sim 0.01-6.38$ with an average value near unity, as seen in other IRDCs (Vasyunina et al. 2011; Liu et al. 2013). It is also in agreement with the theoretical prediction (Sarrasin et al. 2010). The gas kinetic temperature measurements would be needed to study how the $\mathrm{HNC} / \mathrm{HCN}$ ratio depends on the temperature. Moreover, the average $\mathrm{HNC} / \mathrm{HCO}^{+}$and $\mathrm{HN}^{13} \mathrm{C} / \mathrm{H}^{13} \mathrm{CO}^{+}$ratios, $4.3 \pm 3.9$ and $\sim 1.8 \pm 1.1$, are in reasonable agreement with the gasphase chemical models which suggest similar abundances for $\mathrm{HNC}$ and $\mathrm{HCO}^{+}$and their ${ }^{13} \mathrm{C}$ isotopologues in the case of weak ${ }^{13} \mathrm{C}$ fractionation (see e.g. Roberts et al. 2012 and references therein). The median $\mathrm{HNC} / \mathrm{HCO}^{+}$ratio is found to decrease as the clump evolves, in agreement with data from Sanhueza et al. (2012).

5. The $\mathrm{C}_{2} \mathrm{H}(N=1-0)$ emission is also found to be extended. This molecule is believed to be a good tracer of the PDR regions. Towards the IR-binary bubble system N10/11 and the clump G13.22-SMM 32, the detected extended $\mathrm{C}_{2} \mathrm{H}$ emission is probably related to the $\mathrm{UV}$ photodissociation process. Recently, Sanhueza et al. (2013) have found that $\mathrm{C}_{2} \mathrm{H}$ traces cold gas in the IRDC G028.23-00.19, and this also appears to be the case in some of our sources. The fractional $\mathrm{C}_{2} \mathrm{H}$ abundances are found to be comparable to the values obtained by Vasyunina et al. (2011), but the median abundance derived by Sanhueza et al. (2012) exceeds our value by a factor of $\sim 6$. We found that the average and median $\mathrm{C}_{2} \mathrm{H}$ abundances are lower towards more evolved clumps, some of which are likely to be in the hot-core phase or even more evolved. This agrees with the suggestion that $\mathrm{C}_{2} \mathrm{H}$ starts to decrease in abundance in hot cores, and could therefore be used to probe the initial conditions of massive-star formation (Beuther et al. 2008).

6. The $\operatorname{HNCO}\left(4_{0,4}-3_{0,3}\right)$ line emission shows extended morphology towards many of our sources. The average HNCO abundance we derive is a factor of 5.5 times higher than the value obtained by Vasyunina et al. (2011). However, the median value we found is comparable to the one derived by Sanhueza et al. (2012) (within a factor of 1.8). According to models, grain-surface chemistry appears to be required for the origin of gas-phase HNCO (Tideswell et al. 2010). The extended-like emission of HNCO could have its origin in shocks, as suggested in some other molecular clouds (e.g. Zinchenko et al. 2000). In some cases, this is supported by the similarity to the $\mathrm{SiO}$ emission, and also by non-Gaussian line wing emission. Our HNCO data support the discovery that the molecule's column density increases as the clump evolves (Sanhueza et al. 2012); the fractional abundance shows the same trend in the present study.

7. The $J=10-9$ emission of cyanoacetylene $\left(\mathrm{HC}_{3} \mathrm{~N}\right)$ shows extended morphology towards many of our clumps. In some cases the emission maxima correlate well with the dust emission peaks. However, in some sources the $\mathrm{HC}_{3} \mathrm{~N}$ emission is found to be weak or completely absent. The deteced line wings suggest that the $\mathrm{HC}_{3} \mathrm{~N}$ emision has its origin in shocks. On average, we derived an abundance of $\mathrm{HC}_{3} \mathrm{~N}$ that is one order of magnitude higher than Vasyunina et al. (2011) determined for their sources. Our median $\mathrm{HC}_{3} \mathrm{~N}$ abundance is very close to that derived by Sanhueza et al. (2012; within a factor of $\sim 1.4$ ) who found that the column density of $\mathrm{HC}_{3} \mathrm{~N}$ increases as the clump evolves, but our column density data do not show this trend. Only the median abundance shows a hint of positive correlation with the clump evolution. Our results support the finding that, besides tracing hot cores, $\mathrm{HC}_{3} \mathrm{~N}$ can also exist in cold molecular clouds (e.g. in the prestellar core L183; Dickens et al. 2000). The $\mathrm{HC}_{3} \mathrm{~N} / \mathrm{HCN}$ ratios are derived to be $0.02 \pm 0.01-0.24 \pm 0.06$ with an average of $\sim 0.1$. Interestingly, this is similar to what has been detected in low-mass starless cores (Dickens et al. 2000), T Tauri disks (Chapillon et al. 2012), and comets (Bockelée-Morvan et al. 2000).

8. The $\mathrm{CH}_{3} \mathrm{CN}\left(5_{1}-4_{1}\right)$ emission is found to be weak in the three sources where it was detected. The fractional abundance, estimated in these three targets, was found to be $\sim 10^{-11}$ on average. As $\mathrm{CH}_{3} \mathrm{CN}$ is believed to be a hot-core tracer, it is possible that our clumps are mostly too cold (i.e. young) to produce any significant $\mathrm{CH}_{3} \mathrm{CN}$ emission. This agrees with the very low detection rates of $\mathrm{CH}_{3} \mathrm{CN}$ by Vasyunina et al. (2011; no sources) and Sanhueza et al. (2012; one source with active star formation manifested in $4.5 \mu \mathrm{m}$ and $24 \mu \mathrm{m}$ emission). Shock-origin is possible for G1.87-SMM 1 (line-wing emission) and G1.87-SMM 20, 23,30 (peaks at the $\mathrm{SiO}$ maximum).

9. The $J=1-0$ emission of $\mathrm{N}_{2} \mathrm{H}^{+}$is also extended in the clumps we have studied. For example, the line emission traces well the filamentary IRDC G11.36. The $\mathrm{N}_{2} \mathrm{H}^{+}$abundances we derived are comparable to those obtained by Ragan et al. (2006), Vasyunina et al. (2011), and Sanhueza et al. (2012). The correlation found by the last authors and by Hoq et al. (2013), i.e. that the $\mathrm{N}_{2} \mathrm{H}^{+}$abundance increases as the source evolves, is not recognised in our sample. Instead, the opposite trend is manifested in both the average and median abundances. The correlation found by Sanhueza et al. (2012) is also difficult to explain because gas-phase CO should destroy the $\mathrm{N}_{2} \mathrm{H}^{+}$molecules, as discussed by the authors. It could be related to an enhanced $\mathrm{N}_{2}$ abundance, however. The derived $\mathrm{N}_{2} \mathrm{H}^{+} / \mathrm{HCO}^{+}$ratios are in the range $0.14 \pm 0.08-18.57 \pm 3.41$, but no evolutionary trend in this parameter is found. From a theoretical point of view, the $\mathrm{N}_{2} \mathrm{H}^{+} / \mathrm{HCO}^{+}$ratio should decrease when $\mathrm{CO}$ is evaporated off the grain mantles, starting to destroy $\mathrm{N}_{2} \mathrm{H}^{+}$and produce more $\mathrm{HCO}^{+}$. The result by Sanhueza et al. (2012), i.e. that the median $\mathrm{N}_{2} \mathrm{H}^{+} / \mathrm{HCO}^{+}$ratio decreases slightly as the clump continues to evolve from the so-called intermediate stage (associated with an extended $4.5 \mu \mathrm{m}$ emission or a $24 \mu \mathrm{m}$ point source), is in agreement with the above scheme, but in contrast to that of Hoq et al. (2013). The $\mathrm{N}_{2} \mathrm{H}^{+} / \mathrm{HNC}$ is found to be near unity on average in both IR-dark and -bright clumps of our sample. Sanhueza et al. (2012) found generally lower values, while similar values can be calculated from the Liu et al. (2013) data of IRDCs. We found that the $\mathrm{N}_{2} \mathrm{H}^{+} / \mathrm{HNC}$ abundance ratio increases slightly as the clump evolves (our median value changes from 0.72 for IR-dark sources to 1.17 for IR-bright clumps). This is in agreement with the discovery by Sanhueza et al. (2012). Our data suggest that as the $\mathrm{N}_{2} \mathrm{H}^{+}$abundance increases, the abundances of both $\mathrm{HCN}$ and $\mathrm{HNC}$ also increase. This conforms to the small observed change in the $\mathrm{N}_{2} \mathrm{H}^{+} / \mathrm{HNC}$ ratio as the clump evolves further.

Acknowledgements. This paper is dedicated to the memory of S. Miettinen. I would like to thank the anonymous referee for his/her comments and suggestions. The author acknowledges financial support from the Academy of 
Finland through grant 132291. This research has made use of data products from the Millimetre Astronomy Legacy Team $90 \mathrm{GHz}$ (MALT90) survey, NASA's Astrophysics Data System, and the NASA/IPAC Infrared Science Archive, which is operated by the JPL, California Institute of Technology, under contract with NASA. This research made use of APLpy, an open-source plotting package for Python hosted at http://aplpy . gi thub.com.

\section{References}

Allen, T. L., Goddard, J. D., \& Schaefer, H. F., III 1980, J. Chem. Phys., 73, 3255

Araya, E., Hofner, P., Kurtz, S., Bronfman, L., \& DeDeo, S. 2005, ApJS, 157, 279

Argon, A. L., Reid, M. J., \& Menten, K. M. 2000, ApJS, 129, 159

Bachiller, R., \& Perez Gutierrez, M. 1997, ApJ, 487, L93

Battersby, C., Bally, J., Jackson, J. M., et al. 2010, ApJ, 721, 222

Becker, R. H., White, R. L., Helfand, D. J., \& Zoonematkermani, S. 1994, ApJS, 91, 347

Bergin, E. A., \& Tafalla, M. 2007, ARA\&A, 45, 339

Beuther, H., \& Henning, T. 2009, A\&A, 503, 859

Beuther, H., \& Sridharan, T. K. 2007, ApJ, 668, 348

Beuther, H., \& Steinacker, J. 2007, ApJ, 656, L85

Beuther, H., Semenov, D., Henning, T., \& Linz, H. 2008, ApJ, 675, L33

Beuther, H., Linz, H., Tackenberg, J., et al. 2013, A\&A, 553, A115

Bisschop, S. E., Jørgensen, J. K., van Dishoeck, E. F., \& de Wachter, E. B. M. 2007, A\&A, 465, 913

Bockelée-Morvan, D., Lis, D. C., Wink, J. E., et al. 2000, A\&A, 353, 1101

Boger, G. I., \& Sternberg, A. 2005, ApJ, 632, 302

Brown, R. L. 1981, ApJ, 248, L119

Cao, Y. X., Zeng, Q., Deguchi, S., et al. 1993, AJ, 105, 1027

Caselli, P., Benson, P. J., Myers, P. C., \& Tafalla, M. 2002, ApJ, 572, 238

Chambers, E. T., Jackson, J. M., Rathborne, J. M., \& Simon, R. 2009, ApJS, 181, 360

Chapillon, E., Dutrey, A., Guilloteau, S., et al. 2012, ApJ, 756, 58

Chapman, J. F., Millar, T. J., Wardle, M., et al. 2009, MNRAS, 394, 221

Charnley, S. B. 1995, Ap\&SS, 224, 251

Chen, X., Gan, C.-G., Ellingsen, S. P., et al. 2013, ApJS, 206, 22

Chini, R., Kruegel, E., \& Wargau, W. 1987, A\&A, 181, 378

Churchwell, E. 2002, ARA\&A, 40, 27

Churchwell, E., Povich, M. S., Allen, D., et al. 2006, ApJ, 649, 759

Codella, C., Benedettini, M., Beltrán, M. T., et al. 2009, A\&A, 507, L25

Contreras, Y., Schuller, F., Urquhart, J. S., et al. 2013, A\&A, 549, A45

Deharveng, L., Schuller, F., Anderson, L. D., et al. 2010, A\&A, 523, A6

Dickens, J. E., Irvine, W. M., Snell, R. L., et al. 2000, ApJ, 542, 870

Draine, B. T. 2011, Physics of the Interstellar and Intergalactic Medium (Princeton University Press)

Egan, M. P., Shipman, R. F., Price, S. D., et al. 1998, ApJ, 494, L199

Forster, J. R., \& Caswell, J. L. 2000, ApJ, 530, 371

Foster, J. B., Jackson, J. M., Barnes, P. J., et al. 2011, ApJS, 197, 25

Foster, J. B., Rathborne, J. M., Sanhueza, P., et al. 2013, PASA, 30, 38

Fuente, A., Martín-Pintado, J., Cernicharo, J., \& Bachiller, R. 1993, A\&A, 276, 473

Gao, Y., \& Lou, Y.-Q. 2010, MNRAS, 403, 1919

Gao, Y., Lou, Y.-Q., \& Wu, K. 2009, MNRAS, 400, 887

Gardner, F. F., \& Winnewisser, G. 1975, ApJ, 197, L73

Garrod, R. T., Weaver, S. L. W., \& Herbst, E. 2008, ApJ, 682, 283

Gibb, E., Nummelin, A., Irvine, W. M., Whittet, D. C. B., \& Bergman, P. 2000, ApJ, 545, 309

Gibson, D., Plume, R., Bergin, E., et al. 2009, ApJ, 705, 123

Goldsmith, P. F., Irvine, W. M., Hjalmarson, A., \& Ellder, J. 1986, ApJ, 310, 383

Gordon, M. A., \& Sorochenko, R. L. 2009, Radio Recombination Lines - Their Physics and Astronomical Applications (Berlin: Springer), Astrophys. Space Sci. Lib., 282

Guillet, V., Jones, A. P., \& Pineau des Forêts, G. 2009, A\&A, 497, 145

Gusdorf, A., Cabrit, S., Flower, D. R., \& Pineau des Forêts, G. 2008a, A\&A, 482, 809

Gusdorf, A., Pineau des Forêts, G., Cabrit, S., \& Flower, D. R. 2008b, A\&A, 490, 695

Hatchell, J., Thompson, M. A., Millar, T. J., \& MacDonald, G. H. 1998, A\&AS, 133,29

Henshaw, J. D., Caselli, P., Fontani, F., et al. 2013, MNRAS, 428, 3425

Herbst, E. 1978, ApJ, 222, 508

Herbst, E., \& Klemperer, W. 1973, ApJ, 185, 505

Herbst, E., Terzieva, R., \& Talbi, D. 2000, MNRAS, 311, 869

Hirota, T., Yamamoto, S., Mikami, H., \& Ohishi, M. 1998, ApJ, 503, 717

Hoare, M. G., Kurtz, S. E., Lizano, S., Keto, E., \& Hofner, P. 2007, in Protostars and Planets V, eds. B. Reipurth, D. Jewitt, \& K. Keil (Tucson: Univ. of Arizona Press), 181
Hoq, S., Jackson, J. M., Foster, J. B., et al. 2013, ApJ, 777, 157

Iglesias, E. 1977, ApJ, 218, 697

Jackson, J. M., Armstrong, J. T., \& Barrett, A. H. 1984, ApJ, 280, 608

Jackson, J. M., Finn, S. C., Chambers, E. T., et al. 2010, ApJ, 719, L185

Jackson, J. M., Rathborne, J. M., Foster, J. B., et al. 2013, PASA, 30, e057

Jiménez-Serra, I., Caselli, P., Tan, J. C., et al. 2010, MNRAS, 406, 187

Kauffmann, J., \& Pillai, T. 2010, ApJ, 723, L7

Kauffmann, J., Bertoldi, F., Bourke, T. L., et al. 2008, A\&A, 487, 993

Keto, E., \& Rybicki, G. 2010, ApJ, 716, 1315

Klaassen, P. D., Galván-Madrid, R., Peters, T., Longmore, S. N., \& Maercker, M. 2013, A\&A, 556, A107

Kurtz, S., Cesaroni, R., Churchwell, E., Hofner, P., \& Walmsley, C. M. 2000, in Protostars and Planets IV, eds. V. Mannings, A. P. Boss, \& S. S. Russell (Tucson: Univ. of Arizona Press), 299

Ladd, N., Purcell, C., Wong, T., \& Robertson, S. 2005, PASA, 22, 62

Langer, W. D., Graedel, T. E., Frerking, M. A., \& Armentrout, P. B. 1984, ApJ, 277,581

Lapinov, A. V., Golubiatnikov, G. Y., Markov, V. N., \& Guarnieri, A. 2007, Astron. Lett., 33, 121

Lee, C. W., Myers, P. C., \& Tafalla, M. 1999, ApJ, 526, 788

Leurini, S., Codella, C., Zapata, L., et al. 2011, A\&A, 530, A12

Li, J., Wang, J. Z., Gu, Q. S., \& Zheng, X. W. 2013, A\&A, 555, A18

Liu, X.-L., Wang, J.-J., \& Xu, J.-L. 2013, MNRAS, 431, 27

Lo, N., Cunningham, M. R., Jones, P. A., et al. 2009, MNRAS, 395, 1021

MacDonald, G. H., Gibb, A. G., Habing, R. J., \& Millar, T. J. 1996, A\&AS, 119, 333

Mackay, D. D. S. 1999, MNRAS, 304, 61

Martín-Pintado, J., Bachiller, R., \& Fuente, A. 1992, A\&A, 254, 315

Miettinen, O. 2012a, A\&A, 540, A104

Miettinen, O. 2012b, A\&A, 542, A101 (Paper I)

Miettinen, O., Harju, J., Haikala, L. K., \& Pomrén, C. 2006, A\&A, 460, 721

Miettinen, O., Hennemann, M., \& Linz, H. 2011, A\&A, 534, A134

Minh, Y. C., \& Irvine, W. M. 2006, New Astron., 11, 594

Mul, P. M., \& McGowan, J. W. 1980, ApJ, 237, 749

Müller, H. S. P., Schlöder, F., Stutzki, J., \& Winnewisser, G. 2005, J. Mol. Struct., 742, 215

Myers, P. C., Mardones, D., Tafalla, M., Williams, J. P., \& Wilner, D. J. 1996, ApJ, 465, L133

Nomura, H., \& Millar, T. J. 2004, A\&A, 414, 409

Olmi, L., Cesaroni, R., \& Walmsley, C. M. 1996, A\&A, 307, 599

Ossenkopf, V., \& Henning, T. 1994, A\&A, 291, 943

Padovani, M., Walmsley, C. M., Tafalla, M., et al. 2009, A\&A, 505, 1199

Padovani, M., Walmsley, C. M., Tafalla, M., et al. 2011, A\&A, 534, A77

Pagani, L., Daniel, F., \& Dubernet, M.-L. 2009, A\&A, 494, 719

Pandian, J. D., Momjian, E., \& Goldsmith, P. F. 2008, A\&A, 486, 191

Pearson, P. K., \& Schaefer, H. F., III 1974, ApJ, 192, 33

Pérault, M., Omont, A., Simon, G., et al. 1996, A\&A, 315, L165

Peretto, N., \& Fuller, G. A. 2009, A\&A, 505, 405

Pratap, P., Dickens, J. E., Snell, R. L., et al. 1997, ApJ, 486, 862

Purcell, C. R., Balasubramanyam, R., Burton, M. G., et al. 2006, MNRAS, 367, 553

Ragan, S. E., Bergin, E. A., Plume, R., et al. 2006, ApJS, 166, 567

Ragan, S., Henning, T., Krause, O., et al. 2012, A\&A, 547, A49

Rathborne, J. M., Jackson, J. M., \& Simon, R. 2006, ApJ, 641, 389

Rawlings, J. M. C., Taylor, S. D., \& Williams, D. A. 2000, MNRAS, 313, 461

Rawlings, J. M. C., Redman, M. P., Keto, E., \& Williams, D. A. 2004, MNRAS, 351,1054

Reid, M. J., Menten, K. M., Zheng, X. W., et al. 2009, ApJ, 700, 137

Reitblat, A. A. 1980, Sov. Astron. Lett., 6, 406

Rimmer, P. B., Herbst, E., Morata, O., \& Roueff, E. 2012, A\&A, 537, A7

Roberts, J. F., Jiménez-Serra, I., Gusdorf, A., \& Martín-Pintado, J. 2012, A\&A, 544, A 150

Rodríguez-Fernández, N. J., Tafalla, M., Gueth, F., \& Bachiller, R. 2010, A\&A, 516, A98

Sakai, T., Sakai, N., Kamegai, K., et al. 2008, ApJ, 678, 1049

Sakai, T., Sakai, N., Hirota, T., \& Yamamoto, S. 2010, ApJ, 714, 1658

Sanhueza, P., Jackson, J. M., Foster, J. B., et al. 2012, ApJ, 756, 60 (SJF12)

Sanhueza, P., Jackson, J. M., Foster, J. B., et al. 2013, ApJ, 773, 123

Sarrasin, E., Abdallah, D. B., Wernli, M., et al. 2010, MNRAS, 404, 518

Schilke, P., Walmsley, C. M., Pineau des Forêts, G., et al. 1992, A\&A, 256, 595

Schilke, P., Walmsley, C. M., Pineau des Forêts, G., \& Flower, D. R. 1997, A\&A, 321, 293

Schilke, P., Pineau des Forêts, G., Walmsley, C. M., \& Martín-Pintado, J. 2001, A\&A, 372, 291

Schmid-Burgk, J., Muders, D., Müller, H. S. P., \& Brupbacher-Gatehouse, B. 2004, A\&A, 419, 949

Schöier, F. L., van der Tak, F. F. S., van Dishoeck, E. F., \& Black, J. H. 2005, A\&A, 432, 369 
Schuller, F., Menten, K. M. Contreras, Y, et al. 2009, A\&A, 504, 415 Sewilo, M., Watson, C., Araya, E., et al. 2004, ApJS, 154, 553

Simon, R., Jackson, J. M., Rathborne, J. M., \& Chambers, E. T. 2006, ApJ, 639, 227

Snyder, L. E., \& Bhul, D. 1971, BAAS, 3, 388

Spielfiedel, A., Feautrier, N., Najar, F., et al. 2012, MNRAS, 421, 1891

Szymczak, M., Hrynek, G., \& Kus, A. J. 2000, A\&AS, 143, 269

Tackenberg, J., Beuther, H., Henning, T., et al. 2012, A\&A, 540, A113

Takano, S., Masuda, A., Hirahara, Y., et al. 1998, A\&A, 329, 1156

Talbi, D., Ellinger, Y., \& Herbst, E. 1996, A\&A, 314, 688

Thompson, M. A., \& White, G. J. 2004, A\&A, 419, 599

Tideswell, D. M., Fuller, G. A., Millar, T. J., \& Markwick, A. J. 2010, A\&A, 510, A85

Tobin, J. J., Bergin, E. A., Hartmann, L., et al. 2013, ApJ, 765, 18

Townes, C. H., \& Schawlow, A. L. 1975, Microwave Spectroscopy (New York: Dover Publications)

Turner, B. E. 1971, ApJ, 163, L35

Turner, B. E. 1991, ApJS, 76, 617

Turner, B. E., Terzieva, R., \& Herbst, E. 1999, ApJ, 518, 699

Turner, B. E., Herbst, E., \& Terzieva, R. 2000, ApJS, 126, 427
Urquhart, J. S., Hoare, M. G., Purcell, C. R., et al. 2009, A\&A, 501, 539 van Broekhuizen, F. A., Keane, J. V., \& Schutte, W. A. 2004, A\&A, 415, 425 van der Tak, F. F. S., Müller, H. S. P., Harding, M. E., \& Gauss, J. 2009, A\&A, 507,347

van Dishoeck, E. F., \& Blake, G. A. 1998, ARA\&A, 36, 317

van Dishoeck, E. F., Blake, G. A., Jansen, D. J., \& Groesbeck, T. D. 1995, ApJ, 447,760

Vasyunina, T., Linz, H., Henning, T., et al. 2011, A\&A, 527, A88 (VLH11)

Vasyunina, T., Vasyunin, A. I., Herbst, E., \& Linz, H. 2012, ApJ, 751, 105

Velusamy, T., Peng, R., Li, D., et al. 2008, ApJ, 688, L87

Walsh, A. J., Thorwirth, S., Beuther, H., \& Burton, M. G. 2010, MNRAS, 404, 1396

Watson, C., Povich, M. S., Churchwell, E. B., et al. 2008, ApJ, 681, 1341

Watt, G. D., White, G. J., Millar, T. J., \& van Ardenne, A. 1988, A\&A, 195, 257

White, R. L., Becker, R. H., \& Helfand, D. J. 2005, AJ, 130, 586

Wilson, T. L., \& Rood, R. 1994, ARA\&A, 32, 191

Wink, J. E., Altenhoff, W. J., \& Mezger, P. G. 1982, A\&A, 108, 227

Zhang, S. B., Yang, J., Xu, Y., et al. 2011, ApJS, 193, 10

Zhou, S., Evans, N. J., II, Koempe, C., \& Walmsley, C. M. 1993, ApJ, 404, 232

Zinchenko, I., Henkel, C., \& Mao, R. Q. 2000, A\&A, 361, 1079 
O. Miettinen: A MALT90 chemical study of clumps within IRDCs

Table 3. Spectral-line parameters, beam-averaged molecular column densities, and fractional abundances with respect to $\mathrm{H}_{2}$.

\begin{tabular}{|c|c|c|c|c|c|c|c|c|c|}
\hline Source & Transition & $\begin{array}{c}v_{\mathrm{LSR}} \\
{\left[\mathrm{km} \mathrm{s}^{-1}\right]} \\
\end{array}$ & $\begin{array}{c}\Delta v \\
{\left[\mathrm{~km} \mathrm{~s}^{-1}\right]}\end{array}$ & $\begin{array}{c}T_{\mathrm{MB}}{ }^{a} \\
{[\mathrm{~K}]} \\
\end{array}$ & $\begin{array}{l}\int T_{\mathrm{MB}} \mathrm{d} v^{a} \\
{\left[\mathrm{~K} \mathrm{~km} \mathrm{~s}^{-1}\right]}\end{array}$ & $\tau$ & $\begin{array}{c}T_{\mathrm{ex}}{ }^{a} \\
{[\mathrm{~K}]}\end{array}$ & $\begin{array}{c}N^{b} \\
{\left[\mathrm{~cm}^{-2}\right]}\end{array}$ & $\begin{array}{c}x \\
{\left[10^{-10}\right]}\end{array}$ \\
\hline \multirow{21}{*}{$\begin{array}{l}\overline{\text { G1.87-0.14 }} \\
\text { SMM } 1\end{array}$} & & & & & & & & & \\
\hline & $\mathrm{SiO}(2-1)$ & $53.1 \pm 0.5$ & $19.56 \pm 1.02$ & $0.52 \pm 0.24$ & $10.84 \pm 0.50$ & $0.17 \pm 0.01$ & $=E_{\mathrm{u}}=6.25$ & $2.5 \pm 0.1(13)$ & $2.6 \pm 0.3$ \\
\hline & $\mathrm{HN}^{13} \mathrm{C}(1-0)$ & $54.1 \pm 0.6$ & $9.29 \pm 2.67$ & $0.26 \pm 0.21(\mathrm{G})$ & $3.83 \pm 0.40(\mathrm{G})$ & $0.29 \pm 0.03$ & $5.2 \pm 0.1(\mathrm{~A})$ & $9.6 \pm 1.0(12)$ & $1.0 \pm 0.1$ \\
\hline & $\mathrm{C}_{2} \mathrm{H}(1-0)$ & $52.0 \pm 0.3$ & $11.30 \pm 0.71$ & $0.57 \pm 0.23(\mathrm{~S})$ & $8.63 \pm 0.51(\mathrm{~S})$ & $2.38 \pm 1.00$ & $3.8 \pm 0.5$ & $1.2 \pm 0.5(15)$ & $125.4 \pm 53.7$ \\
\hline & $\operatorname{HNCO}\left(4_{0,4}-3_{0,3}\right)$ & $51.6 \pm 0.1$ & $11.70 \pm 0.14$ & $3.04 \pm 0.25(\mathrm{G})$ & $38.68 \pm 0.42(\mathrm{G})$ & $2.86 \pm 0.39$ & $=2 E_{\mathrm{u}} / 3=7.03$ & $7.9 \pm 1.1(14)$ & $82.5 \pm 14.1$ \\
\hline & $\operatorname{HCN}(1-0)^{c}$ & $41.9 \pm 0.4(\mathrm{~B}) /$ & $20.10 \pm 0.89(\mathrm{~B}) /$ & $0.97 \pm 0.37(\mathrm{~B}) /$ & $20.78 \pm 0.80(\mathrm{~B}) /$ & L $\quad \ldots$ & $\ldots$ & $\ldots$ & $\ldots$ \\
\hline & & $62.3 \pm 0.1(\mathrm{R})$ & $11.55 \pm 0.25(\mathrm{R})$ & $2.77 \pm 0.37(\mathrm{R})$ & $34.09 \pm 0.72(\mathrm{R})$ & & & & \\
\hline & $\mathrm{HCO}^{+}(1-0)^{d}$ & $56.8 \pm 0.2$ & $14.76 \pm 0.43$ & $1.58 \pm 0.29$ & $24.84 \pm 0.53$ & $1.67 \pm 0.74$ & $4.9 \pm 0.2(\mathrm{~A})$ & $6.3 \pm 2.8(13)$ & $6.6 \pm 3.0$ \\
\hline & $\mathrm{HCO}^{+}(1-0)^{e, f}$ & $53.8 \pm 0.1$ & $16.13 \pm 0.28$ & $2.24 \pm 0.31$ & $38.38 \pm 0.53$ & $\ldots{ }^{g}$ & $4.9 \pm 0.2(\mathrm{~A})$ & $3.5 \pm 0.1(13)$ & $3.7 \pm 0.4$ \\
\hline & & $-41.5 \pm 0.6$ & $3.37 \pm 1.05$ & $0.52 \pm 0.22$ & $1.87 \pm 0.60$ & $0.31 \pm 0.05$ & $4.9 \pm 0.2(\mathrm{~A})$ & $2.3 \pm 0.7(12)$ & $0.7 \pm 0.2$ \\
\hline & $\operatorname{HNC}(1-0)^{c, d}$ & $52.0 \pm 0.1$ & $10.60 \pm 0.37$ & & & $17.20 \pm 3.60$ & $4.5 \pm 0.1(\mathrm{~A})$ & $5.3 \pm 1.1(14)$ & $55.4 \pm 12.7$ \\
\hline & & $44.3 \pm 0.2(\mathrm{~B}) /$ & $9.47 \pm 0.53(\mathrm{~B}) /$ & $1.21 \pm 0.24(\mathrm{~B}) /$ & $12.24 \pm 0.62(\mathrm{~B}) /$ & & $\ldots$ & & $\ldots$ \\
\hline & & $57.2 \pm 0.1(\mathrm{R})$ & $9.86 \pm 0.29(\mathrm{R})$ & $2.19 \pm 0.24(\mathrm{R})$ & $22.95 \pm 0.62(\mathrm{R})$ & & & & \\
\hline & $\operatorname{HNC}(1-0)^{c, e}$ & $51.8 \pm 0.1$ & $15.40 \pm 0.15$ & & & $1.43 \pm 0.01$ & $4.5 \pm 0.1(\mathrm{~A})$ & $6.4 \pm 0.1(13)$ & $14.7 \pm 1.5$ \\
\hline & & $48.6 \pm 0.4(\mathrm{~B}) /$ & $17.96 \pm 0.48(\mathrm{~B}) /$ & $2.01 \pm 0.20(\mathrm{~B}) /$ & $38.37 \pm 2.03(\mathrm{~B}) /$ & & $\ldots$ & & \\
\hline & & $56.5 \pm 0.1(\mathrm{R})$ & $7.93 \pm 0.46(\mathrm{R})$ & $1.72 \pm 0.20(\mathrm{R})$ & $14.53 \pm 1.77(\mathrm{R})$ & & & & \\
\hline & $\mathrm{HC}_{3} \mathrm{~N}(10-9)$ & $51.6 \pm 0.1$ & $7.74 \pm 0.25$ & $1.66 \pm 0.24(\mathrm{G})$ & $13.92 \pm 0.35(\mathrm{G})$ & $0.23 \pm 0.01$ & $=E_{\mathrm{u}}=24.01$ & $5.8 \pm 0.1(13)$ & $6.1 \pm 0.6$ \\
\hline & $\mathrm{CH}_{3} \mathrm{CN}\left(5_{1}-4_{1}\right)$ & $48.5 \pm 0.4$ & $5.85 \pm 0.69$ & $0.32 \pm 0.23(\mathrm{G})$ & $3.07 \pm 0.31(\mathrm{G})$ & $0.03 \pm 0.01$ & $=2 E_{\mathrm{u}} / 3=13.59$ & $94.2 \pm 0.4(11)$ & $0.04 \pm 0.01$ \\
\hline & $\mathrm{N}_{2} \mathrm{H}^{+}(1-0)^{c}$ & $53.5 \pm 0.3$ & $8.85 \pm 0.53$ & & & $10.70 \pm 1.84$ & $7.2 \pm 0.2(\mathrm{~A})$ & $5.4 \pm 1.0(14)$ & $56.4 \pm 11.8$ \\
\hline & & $46.4 \pm 0.1(\mathrm{~B}) /$ & $13.98 \pm 0.11(\mathrm{~B}) /$ & $0.58 \pm 0.25(\mathrm{~B}) /$ & $8.60 \pm 0.05(\mathrm{~B}) /$ & $\ldots$ & $\ldots$ & $\ldots$ & $\ldots$ \\
\hline & & $60.5 \pm 0.1(\mathrm{R})$ & $9.71 \pm 0.11(\mathrm{R})$ & $1.14 \pm 0.25(\mathrm{R})$ & $11.80 \pm 0.05(\mathrm{R})$ & & & & \\
\hline \multirow[t]{6}{*}{ SMM 8} & $\mathrm{SiO}(2-1)$ & $53.4 \pm 1.0$ & $18.54 \pm 2.48$ & $0.24 \pm 0.22$ & $4.71 \pm 0.53$ & $0.08 \pm 0.01$ & $=E_{\mathrm{u}}=6.25$ & $1.1 \pm 0.1(13)$ & $3.5 \pm 0.5$ \\
\hline & $\operatorname{HNCO}\left(4_{0,4}-3_{0,3}\right)$ & $45.5 \pm 0.5$ & $17.00 \pm 1.52$ & $0.63 \pm 0.23(\mathrm{G})$ & $14.70 \pm 0.61(\mathrm{G})$ & $0.34 \pm 0.02$ & $=2 E_{\mathrm{u}} / 3=7.03$ & $6.1 \pm 0.3(14)$ & $193.2 \pm 21.7$ \\
\hline & $\mathrm{HCN}(1-0)$ & $55.0 \pm 0.1$ & $13.50 \pm 0.38$ & $1.35 \pm 0.26(\mathrm{G})$ & $25.19 \pm 0.57(\mathrm{G})$ & $0.89 \pm 0.10$ & $6.5 \pm 0.8$ & $1.4 \pm 0.2(14)$ & $44.3 \pm 7.8$ \\
\hline & $\mathrm{HCO}^{+}(1-0)$ & $54.1 \pm 0.3$ & $18.17 \pm 0.71$ & $1.06 \pm 0.26$ & $20.45 \pm 0.63$ & $0.79 \pm 0.19$ & $4.9 \pm 0.2(\mathrm{~A})$ & $3.6 \pm 0.9(13)$ & $11.4 \pm 3.1$ \\
\hline & $\mathrm{HNC}(1-0)$ & $49.4 \pm 0.3$ & $17.90 \pm 0.82$ & $1.05 \pm 0.23(\mathrm{G})$ & $20.18 \pm 0.79(\mathrm{G})$ & $2.00 \pm 0.55$ & $4.5 \pm 0.1(\mathrm{~A})$ & $1.0 \pm 0.3(14)$ & $31.7 \pm 10.0$ \\
\hline & $\mathrm{N}_{2} \mathrm{H}^{+}(1-0)$ & $46.6 \pm 0.6$ & $20.10 \pm 1.69$ & $0.47 \pm 0.23(\mathrm{G})$ & $12.90 \pm 0.62(\mathrm{G})$ & $1.26 \pm 0.39$ & $4.6 \pm 1.0$ & $7.2 \pm 3.9(13)$ & $22.8 \pm 12.6$ \\
\hline \multirow[t]{5}{*}{ SMM 10} & $\operatorname{HNCO}\left(4_{0,4}-3_{0,3}\right)$ & $44.3 \pm 0.3$ & $17.00 \pm 0.85$ & $1.14 \pm 0.38(\mathrm{G})$ & $20.88 \pm 0.85$ & $0.67 \pm 0.07$ & $=2 E_{\mathrm{u}} / 3=7.03$ & $2.7 \pm 0.3(14)$ & $188.5 \pm 29.3$ \\
\hline & $\mathrm{HCN}(1-0)$ & $51.7 \pm 0.3$ & $14.30 \pm 0.90$ & $1.38 \pm 0.41$ & $24.74 \pm 0.91$ & $1.87 \pm 0.94$ & $5.1 \pm 0.5(\mathrm{~A})$ & $2.2 \pm 1.1(14)$ & $153.6 \pm 78.6$ \\
\hline & $\mathrm{HCO}^{+}(1-0)$ & $51.8 \pm 0.6$ & $17.33 \pm 1.74$ & $0.61 \pm 0.40$ & $11.35 \pm 0.89$ & $0.38 \pm 0.10$ & $4.9 \pm 0.2(\mathrm{~A})$ & $1.4 \pm 0.1(13)$ & $9.8 \pm 1.3$ \\
\hline & $\mathrm{HNC}(1-0)$ & $46.1 \pm 0.5$ & $18.90 \pm 1.40$ & $1.09 \pm 0.39(\mathrm{G})$ & $22.21 \pm 1.21(\mathrm{G})$ & $2.15 \pm 0.98$ & $4.5 \pm 0.1(\mathrm{~A})$ & $1.2 \pm 0.5(14)$ & $83.8 \pm 36.1$ \\
\hline & $\mathrm{N}_{2} \mathrm{H}^{+}(1-0)$ & $45.7 \pm 0.6$ & $8.33 \pm 1.45$ & $0.64 \pm 0.38(\mathrm{G})$ & $14.68 \pm 0.91(\mathrm{G})$ & $8.08 \pm 3.16$ & $3.6 \pm 0.5$ & $1.4 \pm 2.6(14)$ & $97.7 \pm 181.8$ \\
\hline \multirow[t]{12}{*}{ SMM 12} & $\mathrm{SiO}(2-1)$ & $34.0 \pm 1.5$ & $49.27 \pm 3.32$ & $0.23 \pm 0.20$ & $11.95 \pm 0.72$ & $0.07 \pm 0.01$ & $=E_{\mathrm{u}}=6.25$ & $2.8 \pm 0.2(13)$ & $7.8 \pm 1.0$ \\
\hline & $\mathrm{C}_{2} \mathrm{H}(1-0)$ & $44.6 \pm 0.2$ & $5.67 \pm 0.61$ & $0.40 \pm 0.21(\mathrm{~S})$ & $2.86 \pm 0.27(\mathrm{~S})$ & $4.07 \pm 2.14$ & $3.3 \pm 0.3$ & $8.8 \pm 4.7(14)$ & $244.0 \pm 132.6$ \\
\hline & $\operatorname{HNCO}\left(4_{0,4}-3_{0,3}\right)$ & $44.2 \pm 0.1$ & $8.41 \pm 0.15$ & $2.64 \pm 0.27(\mathrm{G})$ & $24.13 \pm 0.34(\mathrm{G})$ & $2.17 \pm 0.26$ & $=2 E_{\mathrm{u}} / 3=7.03$ & $4.3 \pm 0.5(14)$ & $119.2 \pm 18.3$ \\
\hline & $\operatorname{HCN}(1-0)^{c}$ & $47.4 \pm 0.4$ & $30.40 \pm 0.87$ & & & $\ldots$ & $\ldots$ & $\ldots$ & $\ldots$ \\
\hline & & $41.5 \pm 0.9(\mathrm{~B}) /$ & $37.51 \pm 1.45(\mathrm{~B}) /$ & $0.56 \pm 0.20(\mathrm{~B})$ & $22.48 \pm 1.03(\mathrm{~B}) /$ & & $\ldots$ & $\ldots$ & $\ldots$ \\
\hline & & $54.6 \pm 0.2(\mathrm{R})$ & $9.51 \pm 0.65(\mathrm{R})$ & $0.79 \pm 0.20(\mathrm{R})$ & $8.00 \pm 0.70(\mathrm{R})$ & & & & \\
\hline & $\mathrm{HCO}^{+}(1-0)$ & $44.9 \pm 0.7$ & $41.65 \pm 1.58$ & $0.46 \pm 0.20$ & $20.44 \pm 0.65$ & $0.27 \pm 0.04$ & $4.9 \pm 0.2(\mathrm{~A})$ & $2.5 \pm 0.1(13)$ & $6.9 \pm 0.7$ \\
\hline & $\operatorname{HNC}(1-0)^{c}$ & $46.9 \pm 0.4$ & $22.70 \pm 1.11$ & & & $1.07 \pm 0.22$ & $4.5 \pm 0.1(\mathrm{~A})$ & $7.1 \pm 1.5(13)$ & $19.7 \pm 4.6$ \\
\hline & & $41.1 \pm 0.9(\mathrm{~B}) /$ & $31.45 \pm 1.63(\mathrm{~B}) /$ & $0.41 \pm 0.13(\mathrm{~B}) /$ & $13.77 \pm 0.77(\mathrm{~B}) /$ & $\ldots$ & $\ldots$ & $\ldots$ & \\
\hline & & $50.8 \pm 0.2(\mathrm{R})$ & $6.37 \pm 0.54(\mathrm{R})$ & $0.72 \pm 0.13(\mathrm{R})$ & $4.86 \pm 0.47(\mathrm{R})$ & & & & \\
\hline & $\mathrm{HC}_{3} \mathrm{~N}(10-9)$ & $44.5 \pm 0.1$ & $6.05 \pm 0.28$ & $1.02 \pm 0.21(\mathrm{G})$ & $6.70 \pm 0.28$ & $0.14 \pm 0.01$ & $=E_{\mathrm{u}}=24.01$ & $2.8 \pm 0.1(13)$ & $7.8 \pm 0.8$ \\
\hline & $\mathrm{N}_{2} \mathrm{H}^{+}(1-0)$ & $46.3 \pm 0.5$ & $8.84 \pm 1.21$ & $0.47 \pm 0.20(\mathrm{G})$ & $10.03 \pm 0.50(\mathrm{G})$ & $6.54 \pm 2.19$ & $3.4 \pm 0.3$ & $1.1 \pm 2.0(14)$ & $30.5 \pm 55.5$ \\
\hline \multirow[t]{12}{*}{ SMM 14} & $\mathrm{SiO}(2-1)$ & $38.7 \pm 2.0$ & $50.97 \pm 6.00$ & $0.22 \pm 0.22$ & $12.07 \pm 0.95$ & $0.07 \pm 0.01$ & $=E_{\mathrm{u}}=6.25$ & $2.8 \pm 0.2(13)$ & $5.7 \pm 0.7$ \\
\hline & $\mathrm{C}_{2} \mathrm{H}(1-0)$ & $45.7 \pm 0.5$ & $14.30 \pm 0.70$ & $0.39 \pm 0.21(\mathrm{~S})$ & $6.18 \pm 0.55(\mathrm{~S})$ & $0.94 \pm 0.20$ & $4.1 \pm 0.1(\mathrm{~A})$ & $6.5 \pm 1.3(14)$ & $132.8 \pm 29.7$ \\
\hline & $\operatorname{HNCO}\left(4_{0,4}-3_{0,3}\right)$ & $46.1 \pm 0.1$ & $10.60 \pm 0.20$ & $2.30 \pm 0.26(\mathrm{G})$ & $26.47 \pm 0.41(\mathrm{G})$ & $1.72 \pm 0.17$ & $=2 E_{\mathrm{u}} / 3=7.03$ & $4.3 \pm 0.4(14)$ & $87.9 \pm 12.0$ \\
\hline & $\operatorname{HCN}(1-0)^{c}$ & $46.1 \pm 0.1$ & $41.30 \pm 0.38$ & & & $\ldots$ & $\ldots$ & $\ldots$ & $\ldots$ \\
\hline & & $39.3 \pm 0.2(\mathrm{~B}) /$ & $45.85 \pm 1.6(\mathrm{~B}) /$ & $0.59 \pm 0.22(\mathrm{~B}) /$ & $28.99 \pm 0.98(\mathrm{~B}) /$ & $\ldots$ & $\ldots$ & $\ldots$ & $\ldots$ \\
\hline & & $56.7 \pm 0.2(\mathrm{R})$ & $7.41 \pm 0.55(\mathrm{R})$ & $0.89 \pm 0.22(\mathrm{R})$ & $6.98 \pm 0.43(\mathrm{R})$ & & & & \\
\hline & $\mathrm{HCO}^{+}(1-0)$ & $49.1 \pm 0.8$ & $32.09 \pm 1.72$ & $0.35 \pm 0.21$ & $11.94 \pm 0.58$ & $0.20 \pm 0.03$ & $4.9 \pm 0.2(\mathrm{~A})$ & $1.5 \pm 0.1(13)$ & $3.1 \pm 0.4$ \\
\hline & $\operatorname{HNC}(1-0)^{c}$ & $51.4 \pm 0.2$ & $10.80 \pm 0.61$ & & & $2.78 \pm 0.91$ & $4.5 \pm 0.1(\mathrm{~A})$ & $8.8 \pm 2.9(13)$ & $18.0 \pm 6.2$ \\
\hline & & $44.8 \pm 0.7(\mathrm{~B}) /$ & $26.50 \pm 1.32(\mathrm{~B}) /$ & $0.47 \pm 0.14(\mathrm{~B}) /$ & $13.33 \pm 0.63(\mathrm{~B}) /$ & & & & \\
\hline & & $52.5 \pm 0.1(\mathrm{R})$ & $4.61 \pm 0.25(\mathrm{R})$ & $1.27 \pm 0.14(\mathrm{R})$ & $6.21 \pm 0.36(\mathrm{R})$ & & & & \\
\hline & $\mathrm{HC}_{3} \mathrm{~N}(10-9)$ & $47.2 \pm 0.3$ & $10.40 \pm 0.62$ & $0.56 \pm 0.19(\mathrm{G})$ & $6.28 \pm 0.35(\mathrm{G})$ & $0.07 \pm 0.01$ & $=E_{\mathrm{u}}=24.01$ & $2.6 \pm 0.1(13)$ & $5.3 \pm 0.6$ \\
\hline & $\mathrm{N}_{2} \mathrm{H}^{+}(1-0)$ & $49.8 \pm 0.5$ & $10.70 \pm 2.40$ & $0.56 \pm 0.22(\mathrm{G})$ & $11.41 \pm 0.51$ & $1.85 \pm 0.70$ & $4.4 \pm 0.8$ & $5.3 \pm 3.6(13)$ & $10.8 \pm 5.4$ \\
\hline \multirow[t]{8}{*}{ SMM 15} & $\operatorname{HNCO}\left(4_{0,4}-3_{0,3}\right)$ & $43.2 \pm 0.5$ & $30.40 \pm 1.39$ & $0.63 \pm 0.24(\mathrm{G})$ & $20.76 \pm 0.80(\mathrm{G})$ & $0.34 \pm 0.02$ & $=2 E_{\mathrm{u}} / 3=7.03$ & $1.1 \pm 0.1(14)$ & $48.6 \pm 6.6$ \\
\hline & $\operatorname{HCN}(1-0)^{c}$ & $51.5 \pm 0.5$ & $23.40 \pm 1.24$ & & & 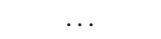 & $\ldots$ & $\ldots$ & 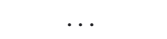 \\
\hline & & $38.5 \pm 0.1(\mathrm{~B}) /$ & $37.71 \pm 0.11(\mathrm{~B}) /$ & $0.23 \pm 0.27(\mathrm{~B}) /$ & $9.41 \pm 0.06(\mathrm{~B}) /$ & & $\ldots$ & & \\
\hline & & $54.5 \pm 0.1(\mathrm{R})$ & $18.49 \pm 0.11(\mathrm{R})$ & $0.79 \pm 0.27(\mathrm{R})$ & $15.64 \pm 0.06(\mathrm{R})$ & $\ldots$ & $\ldots$ & $\ldots$ & $\ldots$ \\
\hline & $\mathrm{HCO}^{+}(1-0)^{c}$ & $27.3 \pm 1.2(\mathrm{~B}) /$ & $14.04 \pm 2.38(\mathrm{~B}) /$ & $0.24 \pm 0.18(\mathrm{~B}) /$ & $3.66 \pm 0.56(\mathrm{~B}) /$ & & $\ldots$ & $\ldots$ & \\
\hline & & $53.7 \pm 0.4(\mathrm{R})$ & $19.26 \pm 1.03(\mathrm{R})$ & $0.75 \pm 0.18(\mathrm{R})$ & $15.43 \pm 0.66(\mathrm{R})$ & & & & \\
\hline & $\mathrm{HNC}(1-0)$ & $43.4 \pm 0.8$ & $21.20 \pm 2.81$ & $0.59 \pm 0.23(\mathrm{G})$ & $18.32 \pm 1.00(\mathrm{G})$ & $3.46 \pm 2.24$ & $3.5 \pm 0.3$ & $1.5 \pm 1.0(14)$ & $66.2 \pm 44.7$ \\
\hline & $\mathrm{N}_{2} \mathrm{H}^{+}(1-0)$ & $39.4 \pm 0.7$ & $17.00 \pm 0.70$ & $0.31 \pm 0.23(\mathrm{G})$ & $12.08 \pm 0.63(\mathrm{G})$ & $0.30 \pm 0.02$ & $7.2 \pm 0.2(\mathrm{~A})$ & $1.6 \pm 0.1(13)$ & $7.1 \pm 0.8$ \\
\hline
\end{tabular}

Notes. ${ }^{(a)}$ "S" refers to the value of the strongest hf component, while "G" refers to the blended group of hf components. The $T_{\text {ex }}$ values marked with "A" represent the average values derived for other sources. ${ }^{(b)} a(b)$ stands for $a \times 10^{b}$. ${ }^{(c)}$ The line is double peaked. Hyperfine fit results are given when possible. Gaussian fit parameters are given separately for both peaks $(\mathrm{B}=$ blue, $\mathrm{R}=\mathrm{red}) .{ }^{(d)}$ Towards the LABOCA peak position. ${ }^{(e)}$ Towards the line emission peak. "N" refers to the northern maximum while " $\mathrm{S}$ " stands for the southern peak. ${ }^{(f)}$ Two or more velocity components. ${ }^{(g)}$ The optical thickness could not be estimated with the assumptions made. The column density was therefore estimated using the assumption of optically thin emission. ${ }^{(h)}$ Calculated from the isotopologue intensity ratio. 
Table 3. continued.

\begin{tabular}{|c|c|c|c|c|c|c|c|c|c|}
\hline Source & Transition & $\begin{array}{c}v_{\mathrm{LSR}} \\
{\left[\mathrm{km} \mathrm{s}^{-1}\right]}\end{array}$ & $\begin{array}{c}\Delta v \\
{\left[\mathrm{~km} \mathrm{~s}^{-1}\right]}\end{array}$ & $\begin{array}{c}T_{\mathrm{MB}}^{a} \\
{[\mathrm{~K}]}\end{array}$ & $\underset{\left[\mathrm{K} \mathrm{km} \mathrm{s}^{-1}\right]}{T_{\mathrm{MB}} \mathrm{d} v^{a}}$ & $\tau$ & $\begin{array}{r}T_{\mathrm{ex}}{ }^{a} \\
{[\mathrm{~K}]}\end{array}$ & $\begin{array}{c}N^{b} \\
{\left[\mathrm{~cm}^{-2}\right]}\end{array}$ & $\begin{array}{c}x \\
{\left[10^{-10}\right]}\end{array}$ \\
\hline \multirow[t]{10}{*}{ SMM 16} & $\mathrm{C}_{2} \mathrm{H}(1-0)$ & $41.5 \pm 0.9$ & $18.90 \pm 2.41$ & $0.24 \pm 0.21(\mathrm{~S})$ & $4.31 \pm 0.48(\mathrm{~S})$ & $0.53 \pm 0.11$ & $4.1 \pm 0.1(\mathrm{~A})$ & $1.7 \pm 0.2(14)$ & $50.8 \pm 7.9$ \\
\hline & $\operatorname{HNCO}\left(4_{0,4}-3_{0,3}\right)$ & $41.7 \pm 0.1$ & $10.80 \pm 0.30$ & $1.33 \pm 0.21(\mathrm{G})$ & $15.51 \pm 0.36(\mathrm{G})$ & $0.81 \pm 0.05$ & $=2 E_{\mathrm{u}} / 3=7.03$ & $2.1 \pm 0.1(14)$ & $62.7 \pm 7.0$ \\
\hline & $\operatorname{HCN}(1-0)^{c}$ & $36.6 \pm 1.1$ & $38.30 \pm 0.54$ & & $\ldots$ & $3.66 \pm 0.33$ & $5.1 \pm 0.5(\mathrm{~A})$ & $1.2 \pm 0.1(15)$ & $358.4 \pm 46.9$ \\
\hline & & $32.5 \pm 1.5(\mathrm{~B}) /$ & $59.84 \pm 2.58(\mathrm{~B}) /$ & $/ 0.36 \pm 0.21(\mathrm{~B}) /$ & $/ 23.09 \pm 1.07(\mathrm{~B}) /$ & $\ldots$ & $\ldots$ & $\ldots$ & $\ldots$ \\
\hline & & $52.8 \pm 0.3(\mathrm{R})$ & $10.48 \pm 0.98(\mathrm{R})$ & $0.54 \pm 0.21(\mathrm{R})$ & $6.03 \pm 0.59(\mathrm{R})$ & $\ldots$ & $\ldots$ & $\ldots$ & $\ldots$ \\
\hline & $\mathrm{HCO}^{+}(1-0)^{c}$ & $13.6 \pm 3.5(\mathrm{~B}) /$ & $32.86 \pm 7.73(\mathrm{~B}) /$ & $0.10 \pm 0.14(\mathrm{~B}) /$ & $3.40 \pm 0.71(\mathrm{~B}) /$ & $\ldots$ & $\ldots$ & $\ldots$ & $\ldots$ \\
\hline & & $48.9 \pm 0.7(\mathrm{R})$ & $19.18 \pm 1.61(\mathrm{R})$ & $0.36 \pm 0.14(\mathrm{R})$ & $7.39 \pm 0.61(\mathrm{R})$ & $\ldots$ & $\ldots$ & $\ldots$ & $\ldots$ \\
\hline & $\mathrm{HNC}(1-0)^{c}$ & $47.0 \pm 0.3$ & $11.30 \pm 1.02$ & $\ldots$ & $\ldots$ & $\ldots$ & $\ldots$ & $\ldots$ & $\ldots$ \\
\hline & & $37.6 \pm 2.8(\mathrm{~B}) /$ & $13.79 \pm 3.82(\mathrm{~B}) /$ & $0.24 \pm 0.21(\mathrm{~B}) /$ & $3.52 \pm 1.20(\mathrm{~B}) /$ & $\ldots$ & $\ldots$ & $\ldots$ & $\ldots$ \\
\hline & & $48.3 \pm 0.5(\mathrm{R})$ & $7.42 \pm 1.05(\mathrm{R})$ & $0.68 \pm 0.21(\mathrm{R})$ & $5.34 \pm 1.24(\mathrm{R})$ & $\ldots$ & $\ldots$ & $\ldots$ & $\ldots$ \\
\hline \multirow[t]{4}{*}{ SMM 20} & $\operatorname{HNCO}\left(4_{0,4}-3_{0,3}\right)$ & $37.4 \pm 0.2$ & $2.90 \pm 0.20$ & $0.38 \pm 0.19(\mathrm{G})$ & $3.03 \pm 0.31(\mathrm{G})$ & $0.20 \pm 0.01$ & $=2 E_{\mathrm{u}} / 3=7.03$ & $1.7 \pm 0.2(13)$ & $6.1 \pm 1.0$ \\
\hline & $\mathrm{HNC}(1-0)$ & $39.2 \pm 0.2$ & $7.16 \pm 0.54$ & $0.71 \pm 0.19(\mathrm{G})$ & $8.60 \pm 0.33(\mathrm{G})$ & $7.30 \pm 2.71$ & $3.6 \pm 0.2$ & $1.1 \pm 0.4(14)$ & $39.4 \pm 14.9$ \\
\hline & $\mathrm{HC}_{3} \mathrm{~N}(10-9)$ & $37.4 \pm 0.6$ & $8.25 \pm 1.36$ & $0.30 \pm 0.25(\mathrm{G})$ & $3.13 \pm 0.38(\mathrm{G})$ & $1.46 \pm 0.68$ & $3.6 \pm 0.7$ & $4.9 \pm 2.4(14)$ & $175.5 \pm 87.8$ \\
\hline & $\mathrm{N}_{2} \mathrm{H}^{+}(1-0)$ & $38.7 \pm 0.4$ & $10.00 \pm 2.47$ & $0.45 \pm 0.21(\mathrm{G})$ & $7.62 \pm 0.40(\mathrm{G})$ & $0.45 \pm 0.03$ & $7.2 \pm 0.2(\mathrm{~A})$ & $9.9 \pm 0.5(12)$ & $3.5 \pm 0.4$ \\
\hline \multirow[t]{8}{*}{ SMM 21} & $\operatorname{HNCO}\left(4_{0,4}-3_{0,3}\right)$ & $40.4 \pm 0.2$ & $11.80 \pm 0.41$ & $1.34 \pm 0.26(\mathrm{G})$ & $17.12 \pm 0.48$ & $0.82 \pm 0.06$ & $=2 E_{\mathrm{u}} / 3=7.03$ & $2.3 \pm 0.2(14)$ & $116.5 \pm 15.7$ \\
\hline & $\operatorname{HCN}(1-0)^{c}$ & $46.9 \pm 0.6$ & $28.90 \pm 1.46$ & & & $\ldots$ & $\ldots$ & $\ldots$ & $\ldots$ \\
\hline & & $30.1 \pm 2.2(\mathrm{~B}) /$ & $19.97 \pm 4.69(\mathrm{~B}) /$ & $0.32 \pm 0.27(\mathrm{~B}) /$ & $6.79 \pm 1.71(\mathrm{~B}) /$ & $\ldots$ & $\ldots$ & $\ldots$ & $\ldots$ \\
\hline & & $51.9 \pm 0.9(\mathrm{R})$ & $18.30 \pm 1.84(\mathrm{R})$ & $0.75 \pm 0.27(\mathrm{R})$ & $14.60 \pm 1.71(\mathrm{R})$ & $\ldots$ & $\ldots$ & $\ldots$ & $\ldots$ \\
\hline & $\mathrm{HCO}^{+}(1-0)^{c}$ & $40.3 \pm 2.3(\mathrm{~B}) /$ & $6.95 \pm 3.76(\mathrm{~B}) /$ & $0.06 \pm 0.27(\mathrm{~B}) /$ & $0.45 \pm 0.50(\mathrm{~B})$ & $\ldots$ & $\ldots$ & $\ldots$ & $\ldots$ \\
\hline & & $55.0 \pm 0.8(\mathrm{R})$ & $30.89 \pm 2.76(\mathrm{R})$ & $0.45 \pm 0.27(\mathrm{R})$ & $14.72 \pm 1.16(\mathrm{R})$ & & & & \\
\hline & $\mathrm{HNC}(1-0)$ & $42.6 \pm 0.5$ & $17.30 \pm 0.86$ & $0.54 \pm 0.25(\mathrm{G})$ & $11.04 \pm 0.58(\mathrm{G})$ & $0.76 \pm 0.15$ & $4.5 \pm 0.1(\mathrm{~A})$ & $3.8 \pm 0.8(13)$ & $19.2 \pm 4.5$ \\
\hline & $\mathrm{N}_{2} \mathrm{H}^{+}(1-0)$ & $41.8 \pm 0.7$ & $16.10 \pm 2.24$ & $0.39 \pm 0.26(\mathrm{G})$ & $9.70 \pm 0.63(\mathrm{G})$ & $2.24 \pm 0.71$ & $3.8 \pm 0.7$ & $7.8 \pm 1.3(13)$ & $39.5 \pm 7.7$ \\
\hline \multirow[t]{15}{*}{ SMM 23} & $\mathrm{SiO}(2-1)$ & $39.2 \pm 0.5$ & $9.65 \pm 1.08$ & $0.34 \pm 0.21$ & $3.50 \pm 0.33$ & $0.11 \pm 0.01$ & $=E_{\mathrm{u}}=6.25$ & $8.2 \pm 0.8(12)$ & $1.6 \pm 0.2$ \\
\hline & $\operatorname{HNCO}\left(4_{0,4}-3_{0,3}\right)^{d}$ & $38.4 \pm 0.5$ & $9.77 \pm 2.28$ & $0.46 \pm 0.21(\mathrm{G})$ & $5.42 \pm 0.38(\mathrm{G})$ & $0.24 \pm 0.013$ & $=2 E_{\mathrm{u}} / 3=7.03$ & $3.0 \pm 0.2(13)$ & $6.0 \pm 0.7$ \\
\hline & $\operatorname{HNCO}\left(4_{0,4}-3_{0,3}\right)^{e}$ & $36.6 \pm 0.1$ & $10.60 \pm 0.29$ & $1.26 \pm 0.22(\mathrm{G})$ & $14.53 \pm 0.37(\mathrm{G})$ & $0.76 \pm 0.05$ & $=2 E_{\mathrm{u}} / 3=7.03$ & $1.9 \pm 0.1(14)$ & $43.4 \pm 5.0$ \\
\hline & $\operatorname{HCN}(1-0)^{c}$ & $32.1 \pm 0.7$ & $23.50 \pm 2.25$ & $\ldots$ & $\ldots$ & $1.02 \pm 0.48$ & $5.1 \pm 0.5(\mathrm{~A})$ & $2.0 \pm 0.9(14)$ & $40.0 \pm 18.5$ \\
\hline & & $22.3 \pm 1.4(\mathrm{~B}) /$ & $34.85 \pm 3.87(\mathrm{~B}) /$ & $/ 0.40 \pm 0.22(\mathrm{~B}) /$ & $14.93 \pm 1.43(\mathrm{~B}) /$ & $\ldots$ & $\ldots$ & $\ldots$ & $\ldots$ \\
\hline & & $47.8 \pm 0.6(\mathrm{R})$ & $15.18 \pm 1.40(\mathrm{R})$ & $0.44 \pm 0.22(\mathrm{R})$ & $7.16 \pm 1.13(\mathrm{R})$ & $\ldots$ & $\ldots$ & $\ldots$ & $\ldots$ \\
\hline & $\mathrm{HCO}^{+}(1-0)$ & $34.7 \pm 0.1$ & $36.23 \pm 0.92$ & $0.24 \pm 0.20$ & $9.18 \pm 0.56$ & $0.13 \pm 0.02$ & $4.9 \pm 0.2(\mathrm{~A})$ & $1.1 \pm 0.1(13)$ & $2.2 \pm 0.3$ \\
\hline & $\mathrm{HNC}(1-0)^{d}$ & $38.0 \pm 0.2$ & $9.97 \pm 0.62$ & $1.17 \pm 0.20(\mathrm{G})$ & $14.41 \pm 0.36(\mathrm{G})$ & $1.32 \pm 0.62$ & $5.2 \pm 0.9$ & $4.8 \pm 2.3(13)$ & $9.6 \pm 4.7$ \\
\hline & $\operatorname{HNC}(1-0)(\mathrm{N})^{e}$ & $37.3 \pm 0.1$ & $10.90 \pm 0.24$ & $1.64 \pm 0.21(\mathrm{G})$ & $19.22 \pm 0.35(\mathrm{G})$ & $\ldots g$ & $4.5 \pm 0.1(\mathrm{~A})$ & $3.4 \pm 0.1(13)$ & $6.8 \pm 0.7$ \\
\hline & $\operatorname{HNC}(1-0)(S)^{e}$ & $37.7 \pm 0.1$ & $9.53 \pm 0.21$ & $1.76 \pm 0.23(\mathrm{G})$ & $18.08 \pm 0.34(\mathrm{G})$ & $\ldots g$ & $4.5 \pm 0.1(\mathrm{~A})$ & $3.2 \pm 0.1(13)$ & $8.0 \pm 0.9$ \\
\hline & $\mathrm{HC}_{3} \mathrm{~N}(10-9)^{d}$ & $38.3 \pm 0.5$ & $6.53 \pm 1.19$ & $0.35 \pm 0.25(\mathrm{G})$ & $3.31 \pm 0.35(\mathrm{G})$ & $0.05 \pm 0.01$ & $=E_{\mathrm{u}}=24.01$ & $1.4 \pm 0.1(13)$ & $2.8 \pm 0.3$ \\
\hline & $\mathrm{HC}_{3} \mathrm{~N}(10-9)^{e}$ & $36.7 \pm 0.1$ & $7.60 \pm 0.37$ & $0.97 \pm 0.26(\mathrm{G})$ & $9.22 \pm 0.392(\mathrm{G})$ & $1.12 \pm 0.10$ & $5.9 \pm 0.8$ & $7.6 \pm 0.8(14)$ & $168.5 \pm 24.4$ \\
\hline & $\mathrm{CH}_{3} \mathrm{CN}\left(5_{1}-4_{1}\right)^{e}$ & $34.8 \pm 0.7$ & $12.20 \pm 1.10$ & $0.25 \pm 0.21(\mathrm{G})$ & $3.94 \pm 0.42(\mathrm{G})$ & $0.02 \pm 0.01$ & $=2 E_{\mathrm{u}} / 3=13.59$ & $5.4 \pm 0.6(11)$ & $0.1 \pm 0.02$ \\
\hline & $\mathrm{N}_{2} \mathrm{H}^{+}(1-0)^{d}$ & $37.9 \pm 0.3$ & $11.60 \pm 0.93$ & $0.57 \pm 0.21(\mathrm{G})$ & $8.93 \pm 0.41(\mathrm{G})$ & $0.57 \pm 0.04$ & $7.2 \pm 0.2(\mathrm{~A})$ & $3.7 \pm 0.4(13)$ & $7.4 \pm 1.1$ \\
\hline & $\mathrm{N}_{2} \mathrm{H}^{+}(1-0)^{e}$ & $36.0 \pm 0.2$ & $8.00 \pm 1.22$ & $1.03 \pm 0.21(\mathrm{G})$ & $15.92 \pm 0.40(\mathrm{G})$ & $1.89 \pm 1.20$ & $5.7 \pm 1.5$ & $5.9 \pm 3.9(13)$ & $14.7 \pm 9.8$ \\
\hline \multirow[t]{8}{*}{ SMM 24} & $\operatorname{HNCO}\left(4_{0,4}-3_{0,3}\right)$ & $43.2 \pm 0.2$ & $11.60 \pm 0.43$ & $1.03 \pm 0.25(\mathrm{G})$ & $13.01 \pm 0.46(\mathrm{G})$ & $0.60 \pm 0.04$ & $=2 E_{\mathrm{u}} / 3=7.03$ & $1.6 \pm 0.1(14)$ & $50.8 \pm 6.1$ \\
\hline & $\mathrm{HCN}(1-0)$ & $43.1 \pm 2.2$ & $26.60 \pm 9.90$ & & & $\ldots$ & $\ldots$ & $\ldots$ & $\ldots$ \\
\hline & & $28.8 \pm 0.1(\mathrm{~B}) /$ & $37.53 \pm 0.11(\mathrm{~B}) /$ & $0.29 \pm 0.26(\mathrm{~B}) /$ & $/ 11.58 \pm 0.05(\mathrm{~B}) /$ & $\ldots$ & $\ldots$ & $\ldots$ & $\ldots$ \\
\hline & & $52.5 \pm 0.1(\mathrm{R})$ & $16.92 \pm 0.11(\mathrm{R})$ & $0.63 \pm 0.26(\mathrm{R})$ & $11.26 \pm 0.05(\mathrm{R})$ & $\ldots$ & $\ldots$ & & $\ldots$ \\
\hline & $\mathrm{HCO}^{+}(1-0)^{c}$ & $37.9 \pm 0.2(\mathrm{~B}) /$ & $40.07 \pm 0.23(\mathrm{~B}) /$ & $0.06 \pm 0.17(\mathrm{~B}) /$ & $2.60 \pm 0.06(\mathrm{~B}) /$ & $\ldots$ & $\ldots$ & $\ldots$ & $\ldots$ \\
\hline & & $55.3 \pm 0.2(\mathrm{R})$ & $30.66 \pm 0.23(\mathrm{R})$ & $0.34 \pm 0.17(\mathrm{R})$ & $11.20 \pm 0.06(\mathrm{R})$ & $\ldots$ & $\ldots$ & $\ldots$ & $\ldots$ \\
\hline & $\mathrm{HNC}(1-0)$ & $43.5 \pm 0.6$ & $12.50 \pm 1.53$ & $0.38 \pm 0.25(\mathrm{G})$ & $6.48 \pm 0.53(\mathrm{G})$ & $3.92 \pm 1.45$ & $3.2 \pm 0.3$ & $9.0 \pm 3.5(13)$ & $28.6 \pm 11.5$ \\
\hline & $\mathrm{N}_{2} \mathrm{H}^{+}(1-0)$ & $44.5 \pm 0.2$ & $11.80 \pm 4.08$ & $0.30 \pm 0.25(\mathrm{G})$ & $7.56 \pm 0.62(\mathrm{G})$ & $0.29 \pm 0.02$ & $7.2 \pm 0.2(\mathrm{~A})$ & $2.3 \pm 1.5(13)$ & $7.3 \pm 4.8$ \\
\hline \multirow[t]{12}{*}{ SMM 27} & $\mathrm{SiO}(2-1)$ & $46.6 \pm 0.6$ & $17.29 \pm 1.12$ & $0.34 \pm 0.20$ & $6.28 \pm 0.41$ & $0.11 \pm 0.01$ & $=E_{\mathrm{u}}=6.25$ & $1.5 \pm 0.1(13)$ & $4.9 \pm 0.6$ \\
\hline & $\mathrm{C}_{2} \mathrm{H}(1-0)$ & $45.0 \pm 0.6$ & $14.90 \pm 1.55$ & $0.24 \pm 0.20(\mathrm{~S})$ & $4.75 \pm 0.43(\mathrm{~S})$ & $0.53 \pm 0.10$ & $4.1 \pm 0.1(\mathrm{~A})$ & $1.7 \pm 0.4(14)$ & $56.1 \pm 14.5$ \\
\hline & $\operatorname{HNCO}\left(4_{0,4}-3_{0,3}\right)$ & $44.5 \pm 0.2$ & $15.20 \pm 1.15$ & $0.79 \pm 0.20(\mathrm{G})$ & $17.26 \pm 0.42(\mathrm{G})$ & $0.44 \pm 0.02$ & $=2 E_{\mathrm{u}} / 3=7.03$ & $9.6 \pm 0.2(13)$ & $31.7 \pm 3.4$ \\
\hline & $\operatorname{HCN}(1-0)^{c}$ & $45.8 \pm 0.5$ & $28.10 \pm 2.33$ & $\ldots$ & & $7.24 \pm 2.98$ & $5.1 \pm 0.5(\mathrm{~A})$ & $1.7 \pm 0.7(15)$ & $560.7 \pm 238.3$ \\
\hline & & $24.6 \pm 0.5(\mathrm{~B}) /$ & $15.46 \pm 1.24(\mathrm{~B}) /$ & $0.42 \pm 0.22(\mathrm{~B}) /$ & $6.84 \pm 0.58(\mathrm{~B})$ & $\ldots$ & $\ldots$ & $\ldots$ & $\ldots$ \\
\hline & & $54.5 \pm 0.4(\mathrm{R})$ & $30.91 \pm 1.09(\mathrm{R})$ & $0.76 \pm 0.22(\mathrm{R})$ & $25.12 \pm 0.74(\mathrm{R})$ & $\ldots$ & $\ldots$ & $\ldots$ & $\ldots$ \\
\hline & $\mathrm{HCO}^{+}(1-0)^{c}$ & $24.0 \pm 1.8(\mathrm{~B}) /$ & $16.35 \pm 2.92(\mathrm{~B}) /$ & $0.13 \pm 0.14(\mathrm{~B}) /$ & $2.23 \pm 0.45(\mathrm{~B})$ & $\ldots$ & $\ldots$ & $\ldots$ & $\ldots$ \\
\hline & & $60.0 \pm 0.6(\mathrm{R})$ & $32.27 \pm 1.95(\mathrm{R})$ & $0.42 \pm 0.14(\mathrm{R})$ & $14.53 \pm 0.68(\mathrm{R})$ & $\ldots$ & $\ldots$ & $\ldots$ & $\ldots$ \\
\hline & HNC(1-0) & $44.2 \pm 0.2$ & $20.20 \pm 0.45$ & $0.92 \pm 0.20(\mathrm{G})$ & $19.95 \pm 0.42(\mathrm{G})$ & $1.60 \pm 0.34$ & $4.5 \pm 0.1(\mathrm{~A})$ & $9.5 \pm 2.0(13)$ & $31.3 \pm 7.4$ \\
\hline & $\mathrm{HC}_{3} \mathrm{~N}(10-9)$ & $46.3 \pm 0.3$ & $15.50 \pm 0.78$ & $0.57 \pm 0.19(\mathrm{G})$ & $9.50 \pm 0.40(G)$ & $0.08 \pm 0.01$ & $=E_{\mathrm{u}}=24.01$ & $4.0 \pm 0.2(13)$ & $13.2 \pm 1.5$ \\
\hline & $\mathrm{CH}_{3} \mathrm{CN}\left(5_{1}-4_{1}\right)$ & $44.5 \pm 0.3$ & $11.50 \pm 4.03$ & $0.20 \pm 0.19(\mathrm{G})$ & $3.08 \pm 0.37(\mathrm{G})$ & $0.02 \pm 0.01$ & $=2 E_{\mathrm{u}} / 3=13.59$ & $7.0 \pm 0.8(11)$ & $0.2 \pm 0.04$ \\
\hline & $\mathrm{N}_{2} \mathrm{H}^{+}(1-0)$ & $45.8 \pm 0.4$ & $18.30 \pm 3.06$ & $0.52 \pm 0.20(\mathrm{G})$ & $12.03 \pm 0.46(\mathrm{G})$ & $0.52 \pm 0.04$ & $7.2 \pm 0.2(\mathrm{~A})$ & $5.4 \pm 1.0(13)$ & $17.8 \pm 3.8$ \\
\hline \multirow[t]{11}{*}{ SMM 28} & $\mathrm{SiO}(2-1)$ & $37.6 \pm 0.4$ & $26.50 \pm 0.83$ & $0.73 \pm 0.21$ & $20.68 \pm 0.58$ & $0.25 \pm 0.02$ & $=E_{\mathrm{u}}=6.25$ & $4.8 \pm 0.1(13)$ & $17.9 \pm 1.9$ \\
\hline & $\mathrm{C}_{2} \mathrm{H}(1-0)$ & $38.2 \pm 0.6$ & $19.20 \pm 1.00$ & $0.28 \pm 0.20(S)$ & $6.16 \pm 0.52(\mathrm{~S})$ & $0.63 \pm 0.12$ & $4.1 \pm 0.1(\mathrm{~A})$ & $2.6 \pm 0.5(14)$ & $97.1 \pm 21.2$ \\
\hline & $\operatorname{HNCO}\left(4_{0,4}-3_{0,3}\right)$ & $39.1 \pm 0.3$ & $21.70 \pm 0.67$ & $0.77 \pm 0.21(\mathrm{G})$ & $18.06 \pm 0.53(\mathrm{G})$ & $0.43 \pm 0.02$ & $=2 E_{\mathrm{u}} / 3=7.03$ & $1.0 \pm 0.1(14)$ & $37.4 \pm 5.4$ \\
\hline & $\operatorname{HCN}(1-0)^{c}$ & $44.5 \pm 0.3$ & $28.90 \pm 0.29$ & & & $23.10 \pm 0.30$ & $5.1 \pm 0.5(\mathrm{~A})$ & $5.5 \pm 0.1(15)$ & $2055 \pm 215.3$ \\
\hline & & $23.4 \pm 0.2(\mathrm{~B}) /$ & $16.03 \pm 0.55(\mathrm{~B}) /$ & $\mid 1.15 \pm 0.27(\mathrm{~B}) /$ & $19.57 \pm 0.78(\mathrm{~B}) /$ & $\ldots$ & $\ldots$ & $\ldots$ & $\ldots$ \\
\hline & & $59.1 \pm 0.6(\mathrm{R})$ & $41.67 \pm 1.60(\mathrm{R})$ & $0.73 \pm 0.27(\mathrm{R})$ & $32.47 \pm 1.01(\mathrm{R})$ & $\cdots$ & $\cdots$ & $\ldots$ & $\cdots$ \\
\hline & $\mathrm{HCO}^{+}(1-0)^{c}$ & $24.2 \pm 0.6(\mathrm{~B}) /$ & $11.34 \pm 1.47(\mathrm{~B}) /$ & $0.27 \pm 0.14(\mathrm{~B})$ & $3.25 \pm 0.41(\mathrm{~B})$ & $\ldots$ & $\ldots$ & & \\
\hline & & $64.9 \pm 0.9(\mathrm{R})$ & $40.80 \pm 2.20(\mathrm{R})$ & $0.37 \pm 0.14(\mathrm{R})$ & $15.89 \pm 0.71(\mathrm{R})$ & & & & \\
\hline & HNC(1-0) & $37.5 \pm 0.2$ & $16.30 \pm 0.81$ & $0.74 \pm 0.21(\mathrm{G})$ & $23.41 \pm 0.51(\mathrm{G})$ & $1.15 \pm 0.22$ & $4.5 \pm 0.1(\mathrm{~A})$ & $5.5 \pm 1.1(13)$ & $20.6 \pm 4.6$ \\
\hline & $\mathrm{HC}_{3} \mathrm{~N}(10-9)$ & $38.3 \pm 0.2$ & $16.00 \pm 0.96$ & $0.97 \pm 0.20(\mathrm{G})$ & $22.26 \pm 0.48(\mathrm{G})$ & $0.13 \pm 0.01$ & $=E_{\mathrm{u}}=24.01$ & $9.3 \pm 0.2(13)$ & $34.7 \pm 3.7$ \\
\hline & $\mathrm{N}_{2} \mathrm{H}^{+}(1-0)$ & $37.7 \pm 0.5$ & $28.70 \pm 1.31$ & $0.56 \pm 0.21(\mathrm{G})$ & $18.35 \pm 0.64(\mathrm{G})$ & $0.52 \pm 0.04$ & $7.2 \pm 0.2(\mathrm{~A})$ & $8.5 \pm 0.8(13)$ & $31.8 \pm 4.4$ \\
\hline SMM 30 & $\mathrm{SiO}(2-1)$ & $39.0 \pm 0.4$ & $10.23 \pm 0.77$ & $0.45 \pm 0.22$ & $4.91 \pm 0.34$ & $0.15 \pm 0.01$ & $=E_{\mathrm{u}}=6.25$ & $1.1 \pm 0.1(13)$ & $3.6 \pm 0.5$ \\
\hline & $\operatorname{HNCO}\left(4_{0,4}-3_{0,3}\right)$ & $38.6 \pm 0.2$ & $10.50 \pm 0.47$ & $0.81 \pm 0.25(\mathrm{G})$ & $9.20 \pm 0.37(\mathrm{G})$ & $0.45 \pm 0.03$ & $=2 E_{\mathrm{u}} / 3=7.03$ & $5.1 \pm 0.2(13)$ & $16.8 \pm 1.9$ \\
\hline & $\operatorname{HCN}(1-0)^{c}$ & $37.9 \pm 0.3$ & $12.90 \pm 0.72$ & & $\ldots$ & $\ldots$ & $\ldots$ & $\ldots$ & $\ldots$ \\
\hline & & $31.4 \pm 0.7(\mathrm{~B}) /$ & $18.81 \pm 1.64(\mathrm{~B}) /$ & $0.64 \pm 0.22(\mathrm{~B}) /$ & $12.84 \pm 0.99(\mathrm{~B})$ & $\ldots$ & & .. & \\
\hline & & $48.6 \pm 0.4(\mathrm{R})$ & $10.24 \pm 0.94(\mathrm{R})$ & $0.77 \pm 0.22(\mathrm{R})$ & $8.41 \pm 0.91(\mathrm{R})$ & $\ldots$ & $\ldots$ & $\ldots$ & \\
\hline
\end{tabular}


Table 3. continued.

\begin{tabular}{|c|c|c|c|c|c|c|c|c|c|}
\hline Source & Transition & $\begin{array}{c}v_{\mathrm{LSR}} \\
{\left[\mathrm{km} \mathrm{s}^{-1}\right]}\end{array}$ & $\begin{array}{c}\Delta v \\
{\left[\mathrm{~km} \mathrm{~s}^{-1}\right]}\end{array}$ & $\begin{array}{c}T_{\mathrm{MB}}{ }^{a} \\
{[\mathrm{~K}]} \\
\end{array}$ & $\begin{array}{c}\int T_{\mathrm{MB}} \mathrm{d} v^{a} \\
{\left[\mathrm{~K} \mathrm{~km} \mathrm{~s}^{-1}\right]}\end{array}$ & $\tau$ & $\begin{array}{c}T_{\mathrm{ex}}{ }^{a} \\
{[\mathrm{~K}]} \\
\end{array}$ & $\begin{array}{c}N^{b} \\
{\left[\mathrm{~cm}^{-2}\right]} \\
\end{array}$ & $\begin{array}{c}x \\
{\left[10^{-10}\right]} \\
\end{array}$ \\
\hline & $\mathrm{HCO}^{+}(1-0)$ & $36.7 \pm 0.9$ & $29.20 \pm 1.95$ & $0.29 \pm 0.21$ & $8.91 \pm 0.56$ & $0.16 \pm 0.02$ & $4.9 \pm 0.2(\mathrm{~A})$ & $1.1 \pm 0.1(13)$ & $3.6 \pm 0.5$ \\
\hline & $\mathrm{HNC}(1-0)$ & $38.5 \pm 0.2$ & $9.24 \pm 0.55$ & $0.98 \pm 0.21(\mathrm{G})$ & $15.67 \pm 0.39(\mathrm{G})$ & $1.77 \pm 0.42$ & $4.5 \pm 0.1(\mathrm{~A})$ & $4.8 \pm 1.2(13)$ & $15.8 \pm 4.3$ \\
\hline & $\mathrm{HC}_{3} \mathrm{~N}(10-9)$ & $39.0 \pm 0.4$ & $5.32 \pm 0.93$ & $0.41 \pm 0.26(\mathrm{G})$ & $3.28 \pm 0.32(\mathrm{G})$ & $0.05 \pm 0.01$ & $=E_{\mathrm{u}}=24.01$ & $1.4 \pm 0.1(13)$ & $4.6 \pm 0.6$ \\
\hline & $\mathrm{N}_{2} \mathrm{H}^{+}(1-0)$ & $39.6 \pm 0.4$ & $9.80 \pm 3.21$ & $0.55 \pm 0.23(\mathrm{G})$ & $9.23 \pm 0.45(\mathrm{G})$ & $0.55 \pm 0.04$ & $7.2 \pm 0.2(\mathrm{~A})$ & $3.1 \pm 1.0(13)$ & $10.2 \pm 3.5$ \\
\hline \multirow[t]{9}{*}{ SMM 31} & $\mathrm{SiO}(2-1)$ & $33.2 \pm 1.1$ & $21.91 \pm 2.44$ & $0.28 \pm 0.26$ & $6.46 \pm 0.66$ & $0.09 \pm 0.01$ & $=E_{\mathrm{u}}=6.25$ & $1.5 \pm 0.2(13)$ & $11.6 \pm 2.0$ \\
\hline & $\operatorname{HNCO}\left(4_{0,4}-3_{0,3}\right)$ & $33.9 \pm 0.3$ & $23.60 \pm 2.03$ & $0.63 \pm 0.27(\mathrm{G})$ & $17.53 \pm 0.70(\mathrm{G})$ & $0.34 \pm 0.02$ & $=2 E_{\mathrm{u}} / 3=7.03$ & $9.7 \pm 0.4(13)$ & $75.3 \pm 8.3$ \\
\hline & $\operatorname{HCN}(1-0)^{c}$ & $28.7 \pm 0.6$ & $37.90 \pm 1.76$ & & & $\ldots$ & & & $\ldots$ \\
\hline & & $26.1 \pm 0.4(\mathrm{~B}) /$ & $26.63 \pm 1.04(\mathrm{~B}) /$ & $0.95 \pm 0.31(\mathrm{~B}) /$ & $26.97 \pm 0.96(\mathrm{~B}) /$ & $\ldots$ & $\ldots$ & & $\ldots$ \\
\hline & & $56.8 \pm 0.9(\mathrm{R})$ & $18.71 \pm 2.36(\mathrm{R})$ & $0.35 \pm 0.31(\mathrm{R})$ & $7.06 \pm 0.67(\mathrm{R})$ & & & & \\
\hline & $\mathrm{HCO}^{+}(1-0)$ & $29.3 \pm 0.6$ & $11.05 \pm 1.91$ & $0.39 \pm 0.28$ & $4.60 \pm 0.59$ & $0.22 \pm 0.04$ & $4.9 \pm 0.2(\mathrm{~A})$ & $5.7 \pm 0.7(12)$ & $4.4 \pm 0.7$ \\
\hline & $\mathrm{HNC}(1-0)$ & $29.4 \pm 0.2$ & $12.50 \pm 0.49$ & $1.19 \pm 0.25(\mathrm{G})$ & $16.11 \pm 0.51(\mathrm{G})$ & $2.57 \pm 0.91$ & $4.5 \pm 0.1(\mathrm{~A})$ & $9.4 \pm 3.3(13)$ & $73.0 \pm 26.7$ \\
\hline & $\mathrm{HC}_{3} \mathrm{~N}(10-9)$ & $33.2 \pm 0.7$ & $22.50 \pm 1.59$ & $0.41 \pm 0.27(\mathrm{G})$ & $9.90 \pm 0.63(\mathrm{G})$ & $0.05 \pm 0.01$ & $=E_{\mathrm{u}}=24.01$ & $4.1 \pm 0.3(13)$ & $31.8 \pm 4.0$ \\
\hline & $\mathrm{N}_{2} \mathrm{H}^{+}(1-0)$ & $31.6 \pm 0.8$ & $19.20 \pm 2.59$ & $0.40 \pm 0.29(\mathrm{G})$ & $9.08 \pm 0.70$ & $0.39 \pm 0.03$ & $7.2 \pm 0.2(\mathrm{~A})$ & $1.2 \pm 0.1(13)$ & $9.3 \pm 1.2$ \\
\hline \multirow[t]{7}{*}{ SMM 38} & $\mathrm{SiO}(2-1)$ & $41.7 \pm 0.6$ & $12.57 \pm 1.53$ & $0.32 \pm 0.27$ & $4.32 \pm 0.45$ & $0.10 \pm 0.01$ & $=E_{\mathrm{u}}=6.25$ & $1.0 \pm 0.1(13)$ & $3.5 \pm 0.5$ \\
\hline & $\operatorname{HNCO}\left(4_{0,4}-3_{0,3}\right)$ & $40.5 \pm 0.9$ & $25.30 \pm 2.61$ & $0.35 \pm 0.25(\mathrm{G})$ & $9.71 \pm 0.75(\mathrm{G})$ & $0.18 \pm 0.01$ & $=2 E_{\mathrm{u}} / 3=7.03$ & $5.4 \pm 0.4(13)$ & $18.8 \pm 2.4$ \\
\hline & $\mathrm{HNC}(1-0)^{d}$ & $42.1 \pm 0.5$ & $15.70 \pm 1.75$ & $0.62 \pm 0.24(\mathrm{G})$ & $12.36 \pm 0.53(\mathrm{G})$ & $1.65 \pm 1.18$ & $3.9 \pm 0.7$ & $6.2 \pm 4.5(13)$ & $21.9 \pm 15.8$ \\
\hline & $\operatorname{HNC}(1-0)^{e}$ & $43.5 \pm 0.4$ & $10.70 \pm 1.17$ & $0.63 \pm 0.25(\mathrm{G})$ & $8.85 \pm 0.44(\mathrm{G})$ & $2.28 \pm 1.40$ & $3.7 \pm 0.5$ & $5.4 \pm 3.4(13)$ & $48.1 \pm 30.8$ \\
\hline & $\mathrm{HC}_{3} \mathrm{~N}(10-9)$ & $40.5 \pm 0.6$ & $9.72 \pm 1.18$ & $0.32 \pm 0.25(\mathrm{G})$ & $3.90 \pm 0.44(\mathrm{G})$ & $0.04 \pm 0.01$ & $=E_{\mathrm{u}}=24.01$ & $7.9 \pm 0.8(14)$ & $274.6 \pm 39.9$ \\
\hline & $\mathrm{N}_{2} \mathrm{H}^{+}(1$ & & $13.40 \pm 1.30$ & $0.56 \pm 0.27(\mathrm{G})$ & $9.85 \pm 0.54(\mathrm{G})$ & $0.56 \pm 0.05$ & $7.2 \pm 0.2(\mathrm{~A})$ & $4.3 \pm 0.6(13)$ & $14.9 \pm 2.6$ \\
\hline & $\mathrm{N}_{2} \mathrm{H}^{+}(1-0)^{e}$ & & $8.49=$ & $7(\mathrm{G})$ & $6.91 \pm 0.46(\mathrm{G})$ & $0.54 \pm 0.05$ & $7.2 \pm 0.2(\mathrm{~A})$ & $2.6 \pm 0.4$ & $21.7 \pm 4.3$ \\
\hline \multicolumn{10}{|l|}{$\mathrm{G} 2.11+0.00$} \\
\hline \multirow[t]{5}{*}{ SMM 5} & $\mathrm{HCN}(1-0)$ & $59.6 \pm 0.2$ & $6.83 \pm 0.86$ & $0.61 \pm 0.20(\mathrm{G})$ & $7.13 \pm 0.40(\mathrm{G})$ & $1.09 \pm 0.21$ & $5.1 \pm 0.5(\mathrm{~A})$ & $6.1 \pm 1.4(13)$ & $22.4 \pm 5.6$ \\
\hline & $\mathrm{HCO}^{+}(1$ & & & & $4.59 \pm$ & & & $7.4 \pm 1$. & \\
\hline & & & & & 0.99 & $0.18 \pm$ & & $1.2 \pm 0$ & \\
\hline & $\mathrm{HNC}(1-0)$ & & & $0.83 \pm 0.20(\mathrm{G})$ & $3.70 \pm$ & & & & $=2.3$ \\
\hline & $\mathrm{N}_{2} \mathrm{H}^{+}(1$ & & & & $1.92 \pm$ & 2.30 & & & $4.8 \pm$ \\
\hline \multicolumn{10}{|l|}{ G11.36+0.80 } \\
\hline \multirow[t]{3}{*}{ SMM 1} & $\mathrm{HCO}^{+}(1-0)$ & & $2.03 \pm 0.36$ & $0.60 \pm 0.24$ & $1.30 \pm 0.19$ & $0.37 \pm 0.07$ & & & \\
\hline & 0) & & & $0.69 \pm 0.33$ & $2.29 \pm 0.31(\mathrm{G})$ & $1.05 \pm 0.29$ & & $9.5 \pm 3$ & \\
\hline & & & & $1.30 \pm 0.28(\mathrm{~S})$ & $3.98 \pm 0.24(\mathrm{~S})$ & $1.46 \pm 0.14$ & $7.2 \pm 0.2(\mathrm{~A})$ & $1.8 \pm 0.3(13)$ & $10.6 \pm 2.1$ \\
\hline \multirow[t]{3}{*}{ SMM 2} & $\mathrm{HCO}^{+}(1-0)$ & & & $0.59 \pm 0.26$ & $1.16 \pm 0.17$ & $0.36 \pm 0.07$ & $4.9 \pm 0.2(\mathrm{~A})$ & $1.4 \pm 0.2(12)$ & $0.6 \pm 0.1$ \\
\hline & $0)$ & & & $0.60 \pm 0.27(\mathrm{G})$ & $2.72 \pm 0.24(\mathrm{G})$ & $0.87 \pm 0.19$ & $4.5 \pm 0.1(\mathrm{~A})$ & $1.0 \pm 0.4$ & $4.2 \pm 1.7$ \\
\hline & & & & $1.61 \pm 0.28(\mathrm{~S})$ & $4.90 \pm 0.26(\mathrm{~S})$ & $1.91 \pm 0.20$ & & $2.6 \pm 0$. & $10.9 \pm 1.7$ \\
\hline \multirow[t]{3}{*}{ SMM 3} & & & & & $0.89=$ & $0.23 \pm 0.04$ & $4.9 \pm$ & $1.1 \pm 0$ & $0.7 \pm 0.1$ \\
\hline & & & & $\pm 0.23(\mathrm{G})$ & $2.18 \pm$ & $0.74 \pm($ & & $5.7 \pm 1$ & $3.4 \pm 1.2$ \\
\hline & & & & & $3.16 \pm$ & $1.26 \pm 1.24$ & & $1.2 \pm 1$ & 7.3 \\
\hline SMM 4 & $\mathrm{HNC}(1-0)$ & & & $0.97 \pm 0.45(\mathrm{G})$ & $2.77 \pm 0.42(\mathrm{G})$ & $1.74 \pm 0.80$ & $4.5 \pm 0.1(\mathrm{~A})$ & $1.3 \pm 0.7(13)$ & $10.8 \pm 5.9$ \\
\hline & $\mathrm{N}_{2} \mathrm{H}^{+}(1-0)$ & & & $1.36 \pm 0.48(\mathrm{G})$ & $3.77 \pm 0.44(\mathrm{G})$ & $1.55 \pm 0.23$ & & $1.5 \pm 0.4(13)$ & $12.4 \pm 3.5$ \\
\hline SMM 5 & $\mathrm{HCO}^{+}(1-0)$ & & & $0.36 \pm 0.23$ & $3.24 \pm 0.43$ & $0.20 \pm 0.03$ & $4.9 \pm 0.2$ & $4.0 \pm 0$. & $2.8 \pm 0.5$ \\
\hline & & & & $0.56 \pm 0.28(\mathrm{G})$ & $1.79 \pm 0.20(\mathrm{G})$ & $0.80 \pm 0.18$ & $4.5 \pm$ & $4.0 \pm 0$. & $2.8 \pm 0.7$ \\
\hline & & & & & $2.77 \pm$ & $3.17 \pm$ & & & $12.0 \pm 7.8$ \\
\hline SMM 6 & & & & & $1.77 \pm 0.38$ & $0.11 \pm 0.02$ & & $2.2 \pm 0$. & $3.4 \pm 0.9$ \\
\hline & & $.7 \pm 0.1$ & $7 \pm 0.10$ & $0.56 \pm 0.31(\mathrm{G})$ & $1.76 \pm 0.21(\mathrm{G})$ & $0.80 \pm 0.19$ & $4.5 \pm 0.1(\mathrm{~A})$ & $3.7 \pm 0.9(12)$ & $5.8 \pm 1.6$ \\
\hline & & & & & $1.59 \pm$ & 48 & & $1.6 \pm 2$ & 43.9 \\
\hline SMM 7 & $\mathrm{HCO}^{+}(1-0)$ & & & $83 \pm 0.31$ & $1.97 \pm 0.21$ & $0.56 \pm 0$ & & $3.2 \pm 0.9$ & $4.7 \pm 1.4$ \\
\hline & & & & $0.63 \pm 0.25(\mathrm{G})$ & $2.00 \pm 0.26(\mathrm{G})$ & $0.93 \pm 0.19$ & $4.5 \pm 0.1(\mathrm{~A})$ & $7.9 \pm 2.2(12)$ & $11.7 \pm 3.5$ \\
\hline & $\mathrm{N}_{2} \mathrm{H}^{+}(1-0)$ & $28.2 \pm 0.1$ & $0.79 \pm 0.26$ & $0.58 \pm 0.23(\mathrm{~S})$ & $1.38 \pm 0.20(\mathrm{~S})$ & $3.84 \pm 3.74$ & $3.8 \pm 0.7$ & $6.6 \pm 12.9(12)$ & $9.8 \pm 19.1$ \\
\hline & & & & & & & & & \\
\hline & & & & & $8.75 \pm$ & & & & \\
\hline & & & & & & & & & \\
\hline & & & & & $1.03 \pm$ & $0.30 \pm 0.05$ & & $1.3 \pm 0.2$ & 0.1 \\
\hline & & & & & $6.17 \pm$ & & & (13) & $=0.8$ \\
\hline & & $9.8 \pm 0.1$ & $7 \pm 0.17$ & $54 \pm 0.26(\mathrm{~S})$ & $6.02 \pm 0.32(\mathrm{~S})$ & $1.81 \pm 0.18$ & $7.2 \pm 0.2(\mathrm{~A})$ & $2.8 \pm 0.3(13)$ & $8.2 \pm 1.2$ \\
\hline SMM 5 & $\mathrm{H}^{13} \mathrm{CO}^{+}(1-0)$ & $49.0 \pm 0.2$ & $2.74 \pm 0.50$ & $0.61 \pm 0.24(\mathrm{G})$ & $1.83 \pm 0.27$ & $0.65_{-029}^{+0.31 h}$ & $5.6 \pm 1.5$ & $5.8 \pm 2.9(12)$ & $0.4 \pm 0.2$ \\
\hline & & $48.8 \pm 0.4$ & $5.95 \pm 1.20$ & $0.38 \pm 0.24$ & $2.38 \pm 0.35$ & $0.12 \pm 0.01$ & $=E_{\mathrm{u}}=6.25$ & $5.5 \pm 0.8(12)$ & $0.4 \pm 0.1$ \\
\hline & $\mathrm{HN}^{13} \mathrm{C}(1-0)$ & $50.0 \pm 0.2$ & $2 \pm 0.39$ & $0.66 \pm 0.25(\mathrm{G})$ & $2.54 \pm 0.24(\mathrm{G})$ & $0.69_{-030}^{+0.40 h}$ & $5.4 \pm 1.4$ & $1.5 \pm 0.8(13)$ & $1.2 \pm 0.6$ \\
\hline & $\mathrm{C}_{2} \mathrm{H}(1-0)^{d}$ & & & & $3.71 \pm 0.32(\mathrm{~S})$ & $2.08 \pm 0.68$ & $0.1(\mathrm{~A})$ & $4.5 \pm 1.3(14)$ & $34.7 \pm 10.6$ \\
\hline & $\mathrm{C}_{2} \mathrm{H}(1-0)^{e}$ & $0.1 \pm 0.1$ & $4.09 \pm 0.32$ & $0.82 \pm 0.21(\mathrm{~S})$ & $3.81 \pm 0.32(\mathrm{~S})$ & $1.17 \pm 0.04$ & $5.1 \pm 0.6$ & $3.4 \pm 0.3(14)$ & $40.9 \pm 5.4$ \\
\hline & $\mathrm{HCN}(1-0)$ & $47.4 \pm 0.3$ & $16.20 \pm 0.79$ & $1.45 \pm 0.34(\mathrm{~S})$ & $7.28 \pm 0.38(\mathrm{~S})$ & $0.58 \pm 0.07^{d}$ & $5.1 \pm 0.5(\mathrm{~A})$ & $7.7 \pm 1.0(13)$ & $5.9 \pm 1.0$ \\
\hline & $\mathrm{HCO}^{+}(1-0)$ & $48.2 \pm 0.1$ & $4.46 \pm 0.14$ & $2.60 \pm 0.25$ & $12.34 \pm 0.30$ & $10.90_{-480}^{+5.30 h}$ & $5.6 \pm 0.3$ & $1.5 \pm 0.7(14)$ & $11.6 \pm 5.5$ \\
\hline & $\operatorname{HNC}(1-0)^{f}$ & $49.1 \pm 0.1$ & $4.65 \pm 0.37$ & $2.55 \pm 0.26(\mathrm{G})$ & $14.19 \pm 0.29(\mathrm{G})$ & $21.78^{+10.80 h}$ & $5.5 \pm 0.3$ & $2.2 \pm 1.0(14)$ & $17.0 \pm 7.9$ \\
\hline & & & & & & & & & \\
\hline & & & & & & & $=E_{\mathrm{u}}=$ & & \\
\hline & ${ }_{2} \mathrm{H}^{+}(1-0)$ & $49.3 \pm 0.1$ & $3.43 \pm 0.07$ & $3.89 \pm 0.30(\mathrm{~S})$ & $18.10 \pm 0.43(\mathrm{~S})$ & $1.19 \pm 0.28$ & $17.9 \pm 3.2$ & $1.1 \pm 0.3(14)$ & $8.5 \pm 2.5$ \\
\hline SMM 6 & & & & $0.76 \pm 0.38(\mathrm{G})$ & $11.23 \pm 0.84(\mathrm{G})$ & $0.79 \pm 0.28$ & $5.1 \pm 0.5(\mathrm{~A})$ & $2.8 \pm 1.1(13)$ & $12.4 \pm 5.0$ \\
\hline & $\mathrm{HCO}^{+}(1-0)$ & $46.9 \pm 0.1$ & $3 \pm 0.32$ & $1.94 \pm 0.38$ & $5.64 \pm 0.42$ & $\ldots^{g}$ & $4.9 \pm 0.2(\mathrm{~A})$ & $6.9 \pm 0.5(12)$ & \\
\hline & $\mathrm{HNC}(1-0)$ & $9.6 \pm 0.1$ & $2.60 \pm 0.23$ & $1.86 \pm 0.37(\mathrm{G})$ & $5.33 \pm 0.34(\mathrm{G})$ & $\ldots{ }^{g}$ & $4.5 \pm 0.1(\mathrm{~A})$ & $9.4 \pm 0.6(12)$ & $4.2 \pm 0.5$ \\
\hline & & $49.4 \pm 0.1$ & & $1.62 \pm 0.44(\mathrm{~S})$ & $5.17 \pm 0.40(\mathrm{~S})$ & $1.93 \pm 0.28$ & $7.2 \pm 0.2(\mathrm{~A})$ & $6.9 \pm 0.4(12)$ & $3.1 \pm 0.4$ \\
\hline SMM 7 & $\mathrm{HN}^{13} \mathrm{C}(1-0)$ & $52.2 \pm 0.1$ & & $0.72 \pm 0.20(\mathrm{G})$ & $1.87 \pm 0.16(\mathrm{G})$ & $0.79_{-0.26}^{+0.30 h}$ & $5.5 \pm 1.1$ & $1.1 \pm 0.2(13)$ & $1.2 \pm 0.3$ \\
\hline & & & & $1.02 \pm 0.23(\mathrm{~S})$ & $3.32 \pm 0.23(\mathrm{~S})$ & $1.62 \pm 0.94$ & & $2.9 \pm 1.7(14)$ & $32.1 \pm 19.1$ \\
\hline & $\mathrm{HCN}(1-0)$ & $51.5 \pm 0.1$ & $3.21 \pm 0.13$ & $1.64 \pm 0.30(\mathrm{~S})$ & $6.41 \pm 0.12$ & $1.96 \pm 0.58$ & $5.5 \pm 0.6$ & $5.8 \pm 1.7(13)$ & $6.4 \pm 2.0$ \\
\hline & $\mathrm{HCO}^{+}(1-0)^{f}$ & $51.1 \pm 0.1$ & $5.70 \pm 0.23$ & $1.62 \pm 0.28$ & $9.80 \pm 0.29$ & $1.79 \pm 0.83$ & $4.9 \pm 0.2(\mathrm{~A})$ & $2.6 \pm 1.2(13)$ & $2.9 \pm 1.4$ \\
\hline
\end{tabular}


A\&A 562, A3 (2014)

Table 3. continued.

\begin{tabular}{|c|c|c|c|c|c|c|c|c|c|}
\hline Source & Transition & $\begin{array}{c}v_{\mathrm{LSR}} \\
{\left[\mathrm{km} \mathrm{s}^{-1}\right]}\end{array}$ & $\begin{array}{c}\Delta v \\
{\left[\mathrm{~km} \mathrm{~s}^{-1}\right]}\end{array}$ & $\begin{array}{c}T_{\mathrm{MB}}{ }^{a} \\
{[\mathrm{~K}]}\end{array}$ & $\underset{\left[\mathrm{K} \mathrm{km} \mathrm{s}^{-1}\right]}{T_{\mathrm{MB}} \mathrm{d} v^{a}}$ & $\tau$ & $\begin{array}{r}T_{\mathrm{ex}}{ }^{a} \\
{[\mathrm{~K}]}\end{array}$ & $\begin{array}{c}N^{b} \\
{\left[\mathrm{~cm}^{-2}\right]}\end{array}$ & $\begin{array}{c}x \\
{\left[10^{-10}\right]}\end{array}$ \\
\hline & & $37.0 \pm 0.1$ & $2.05 \pm 0.38$ & $0.57 \pm 0.28$ & $1.25 \pm 0.17$ & $0.35 \pm 0.07$ & $4.9 \pm 0.2(\mathrm{~A})$ & $1.5 \pm 0.2(12)$ & $0.2 \pm 0.03$ \\
\hline & $\operatorname{HNC}(1-0)^{f}$ & $51.5 \pm 0.1$ & $3.82 \pm 0.37$ & $2.49 \pm 0.23(\mathrm{G})$ & $10.92 \pm 0.22$ & $24.84_{-8.10}^{+9.36 h}$ & $5.5 \pm 0.2$ & $3.7 \pm 1.4(14)$ & $41.0 \pm 16.0$ \\
\hline & & $36.8 \pm 0.1$ & $2.27 \pm 0.37$ & $0.37 \pm 0.23(\mathrm{G})$ & $1.00 \pm 0.16(\mathrm{G})$ & $1.10 \pm 0.10$ & $3.7 \pm 0.6$ & $5.5 \pm 1.0(12)$ & $0.6 \pm 0.1$ \\
\hline & $\mathrm{N}_{2} \mathrm{H}^{+}(1-0)$ & $51.7 \pm 0.1$ & $2.59 \pm 0.06$ & $3.83 \pm 0.32(\mathrm{~S})$ & $13.86 \pm 0.40(\mathrm{~S})$ & $1.00 \pm 0.28$ & $20.0 \pm 4.4$ & $8.9 \pm 2.5(13)$ & $9.9 \pm 2.9$ \\
\hline \multirow[t]{5}{*}{ SMM 11} & $\mathrm{HCO}^{+}(1-0)^{f}$ & $53.5 \pm 0.3$ & $8.64 \pm 0.67$ & $0.56 \pm 0.23$ & $5.10 \pm 0.36$ & $0.34 \pm 0.06$ & $4.9 \pm 0.2(\mathrm{~A})$ & $6.3 \pm 0.5(12)$ & $3.3 \pm 0.4$ \\
\hline & & $37.2 \pm 0.1$ & $1.73 \pm 0.30$ & $0.60 \pm 0.23$ & $1.10 \pm 0.17$ & $0.37 \pm 0.07$ & $4.9 \pm 0.2(\mathrm{~A})$ & $1.4 \pm 0.2(12)$ & $0.7 \pm 0.1$ \\
\hline & $\mathrm{HNC}(1-0)^{f}$ & $52.5 \pm 0.1$ & $4.26 \pm 0.32$ & $0.88 \pm 0.24(\mathrm{G})$ & $4.09 \pm 0.25(\mathrm{G})$ & $1.49 \pm 0.36$ & $4.5 \pm 0.1(\mathrm{~A})$ & $1.9 \pm 0.5(13)$ & $9.9 \pm 2.8$ \\
\hline & & $37.8 \pm 0.1$ & $1.26 \pm 0.16$ & $0.32 \pm 0.24(\mathrm{G})$ & $0.85 \pm 0.19(\mathrm{G})$ & $0.41 \pm 0.07$ & $4.5 \pm 0.1(\mathrm{~A})$ & $1.5 \pm 0.3(12)$ & $0.8 \pm 0.2$ \\
\hline & $\mathrm{N}_{2} \mathrm{H}^{+}(1-0)$ & $52.3 \pm 0.1$ & $2.45 \pm 0.30$ & $0.90 \pm 0.27(\mathrm{~S})$ & $3.27 \pm 0.26(\mathrm{~S})$ & $1.87 \pm 1.63$ & $5.3 \pm 1.8$ & $1.6 \pm 1.4(13)$ & $8.3 \pm 7.4$ \\
\hline \multirow[t]{7}{*}{ SMM 23} & $\mathrm{HCN}(1-0)$ & $37.1 \pm 0.1$ & $2.82 \pm 0.29$ & $0.42 \pm 0.24(\mathrm{G})$ & $4.64 \pm 0.37(G)$ & $2.30 \pm 1.62$ & $3.4 \pm 0.4$ & $3.0 \pm 2.1(13)$ & $18.5 \pm 13.1$ \\
\hline & $\mathrm{HCO}^{+}(1-0)^{f}$ & $37.0 \pm 0.1$ & $3.25 \pm 0.18$ & $1.39 \pm 0.23$ & $4.81 \pm 0.22$ & $1.26 \pm 0.37$ & $4.9 \pm 0.2(\mathrm{~A})$ & $1.0 \pm 0.3(13)$ & $6.2 \pm 2.0$ \\
\hline & & $10.4 \pm 0.2$ & $1.72 \pm 0.44$ & $0.35 \pm 0.22$ & $0.64 \pm 0.15$ & $0.20 \pm 0.03$ & $4.9 \pm 0.2(\mathrm{~A})$ & $7.9 \pm 1.8(11)$ & $0.5 \pm 0.1$ \\
\hline & & $53.0 \pm 0.2$ & $3.50 \pm 0.55$ & $0.42 \pm 0.22$ & $1.55 \pm 0.21$ & $0.24 \pm 0.03$ & $4.9 \pm 0.2(\mathrm{~A})$ & $1.9 \pm 0.3(12)$ & $1.2 \pm 0.2$ \\
\hline & $\operatorname{HNC}(1-0)^{f}$ & $37.7 \pm 0.1$ & $3.45 \pm 0.14$ & $1.50 \pm 0.20(\mathrm{G})$ & $5.62 \pm 0.20(\mathrm{G})$ & $5.74 \pm 5.54$ & $4.5 \pm 0.1(\mathrm{~A})$ & $5.8 \pm 5.6(13)$ & $35.7 \pm 34.7$ \\
\hline & & $52.7 \pm 0.2$ & $3.14 \pm 0.20$ & $0.30 \pm 0.20(\mathrm{G})$ & $1.82 \pm 0.25(\mathrm{G})$ & $0.38 \pm 0.06$ & $4.5 \pm 0.1(\mathrm{~A})$ & $3.2 \pm 0.4(12)$ & $2.0 \pm 0.3$ \\
\hline & $\mathrm{N}_{2} \mathrm{H}^{+}(1-0)$ & $38.2 \pm 0.1$ & $2.29 \pm 0.17$ & $1.43 \pm 0.18(\mathrm{~S})$ & $4.46 \pm 0.24(S)$ & $1.02 \pm 0.77$ & $9.2 \pm 4.2$ & $2.0 \pm 1.5(13)$ & $12.3 \pm 9.3$ \\
\hline \multirow[t]{10}{*}{ SMM 27} & $\mathrm{H}^{13} \mathrm{CO}^{+}(1-0)$ & $36.6 \pm 0.1$ & $2.97 \pm 0.31$ & $0.72 \pm 0.21(\mathrm{G})$ & $2.31 \pm 0.21(\mathrm{G})$ & $1.51_{-0.62}^{+0.80 h}$ & $4.5 \pm 0.7$ & $1.0 \pm 0.5(13)$ & $1.4 \pm 0.7$ \\
\hline & $\mathrm{HN}^{13} \mathrm{C}(1-0)$ & $37.2 \pm 0.1$ & $1.73 \pm 0.57$ & $0.71 \pm 0.22(\mathrm{G})$ & $1.53 \pm 0.18$ & $1.09_{-0.42}^{+0.51 h}$ & $4.8 \pm 0.9$ & $1.0 \pm 0.5(13)$ & $1.4 \pm 0.7$ \\
\hline & $\mathrm{C}_{2} \mathrm{H}(1-0)$ & $36.8 \pm 0.1$ & $2.64 \pm 0.28$ & $0.61 \pm 0.20(S)$ & $1.577 \pm 0.18(\mathrm{~S})$ & $4.01 \pm 2.44$ & $3.6 \pm 0.3$ & $4.6 \pm 2.8(14)$ & $63.8 \pm 39.3$ \\
\hline & $\mathrm{HCN}(1-0)$ & $36.1 \pm 0.5$ & $15.90 \pm 1.92$ & $0.52 \pm 0.25(\mathrm{G})$ & $10.15 \pm 0.68(\mathrm{G})$ & $0.88 \pm 0.20$ & $5.1 \pm 0.5(\mathrm{~A})$ & $1.2 \pm 0.3(14)$ & $16.6 \pm 4.5$ \\
\hline & $\mathrm{HCO}^{+}(1-0)$ & $36.2 \pm 0.1$ & $4.05 \pm 0.17$ & $1.55 \pm 0.24$ & $6.70 \pm 0.23$ & $28.30_{-1140}^{+15.10 h}$ & $4.5 \pm 0.3$ & $2.6 \pm 1.2(14)$ & $36.1 \pm 17.0$ \\
\hline & $\mathrm{HNC}(1-0)$ & $36.7 \pm 0.1$ & $3.54 \pm 0.10$ & $1.91 \pm 0.23(\mathrm{G})$ & $7.72 \pm 0.23(\mathrm{G})$ & $37.80^{+11.82 h}$ & $4.9 \pm 0.2$ & $4.4 \pm 1.9(14)$ & $61.0 \pm 27.0$ \\
\hline & $\mathrm{HC}_{3} \mathrm{~N}(10-9)^{d}$ & $36.7 \pm 0.2$ & $2.73 \pm 0.33$ & $0.57 \pm 0.21(\mathrm{G})$ & $1.67 \pm 0.19(\mathrm{G})$ & $0.08 \pm 0.01$ & $=E_{\mathrm{u}}=24.01$ & $7.0 \pm 0.8(12)$ & $1.0 \pm 0.1$ \\
\hline & $\mathrm{HC}_{3} \mathrm{~N}(10-9)^{e}$ & $36.7 \pm 0.1$ & $3.29 \pm 0.32$ & $0.63 \pm 0.21(\mathrm{G})$ & $2.23 \pm 0.20(\mathrm{G})$ & $0.08 \pm 0.01$ & $=E_{\mathrm{u}}=24.01$ & $9.3 \pm 0.8(12)$ & $2.0 \pm 0.2$ \\
\hline & $\mathrm{N}_{2} \mathrm{H}^{+}(1-0)^{d}$ & $36.8 \pm 0.1$ & $2.77 \pm 0.12$ & $2.01 \pm 0.22(\mathrm{~S})$ & $7.81 \pm 0.28(\mathrm{~S})$ & $1.85 \pm 0.55$ & $8.4 \pm 1.4$ & $3.7 \pm 1.1(13)$ & $5.1 \pm 1.6$ \\
\hline & $\mathrm{N}_{2} \mathrm{H}^{+}(1-0)^{e}$ & $36.7 \pm 0.1$ & $2.82 \pm 0.09$ & $2.39 \pm 0.21(\mathrm{~S})$ & $9.99 \pm 0.31(\mathrm{~S})$ & $2.58 \pm 0.47$ & $8.0 \pm 0.8$ & $4.9 \pm 0.9(13)$ & $9.0 \pm 1.9$ \\
\hline \multirow[t]{14}{*}{ SMM 29} & $\mathrm{C}_{2} \mathrm{H}(1-0)$ & $45.4 \pm 0.1$ & $1.77 \pm 0.29$ & $0.49 \pm 0.18(\mathrm{~S})$ & $1.31 \pm 0.20(\mathrm{~S})$ & $1.25 \pm 0.26$ & $4.1 \pm 0.1(\mathrm{~A})$ & $1.1 \pm 0.3(14)$ & $18.3 \pm 5.3$ \\
\hline & $\mathrm{HCN}(1-0)$ & $57.7 \pm 0.2$ & $2.41 \pm 0.46$ & $0.49 \pm 0.22(\mathrm{G})$ & $1.21 \pm 0.18(\mathrm{G})$ & $0.82 \pm 0.16$ & $5.1 \pm 0.5(\mathrm{~A})$ & $1.6 \pm 0.4(13)$ & $2.7 \pm 0.7$ \\
\hline & $\mathrm{HCO}^{+}(1-0)^{d, f}$ & $44.8 \pm 0.1$ & $3.60 \pm 0.11$ & $1.23 \pm 0.23$ & $4.72 \pm 0.04$ & $1.00 \pm 0.25$ & $4.9 \pm 0.2(\mathrm{~A})$ & $9.2 \pm 2.3(12)$ & $1.5 \pm 0.4$ \\
\hline & & $13.6 \pm 0.1$ & $1.96 \pm 0.11$ & $0.37 \pm 0.23$ & $0.77 \pm 0.04$ & $0.21 \pm 0.03$ & $4.9 \pm 0.2(\mathrm{~A})$ & $9.5 \pm 0.5(11)$ & $0.2 \pm 0.02$ \\
\hline & & $34.8 \pm 0.1$ & $5.43 \pm 0.11$ & $0.34 \pm 0.23$ & $1.97 \pm 0.04$ & $0.19 \pm 0.03$ & $4.9 \pm 0.2(\mathrm{~A})$ & $2.4 \pm 0.1(12)$ & $0.4 \pm 0.04$ \\
\hline & & $57.0 \pm 0.1$ & $3.65 \pm 0.11$ & $0.24 \pm 0.23$ & $0.93 \pm 0.04$ & $0.13 \pm 0.02$ & $4.9 \pm 0.2(\mathrm{~A})$ & $1.1 \pm 0.1(12)$ & $0.2 \pm 0.02$ \\
\hline & $\mathrm{HCO}^{+}(1-0)^{e}$ & $44.6 \pm 0.1$ & $2.44 \pm 0.13$ & $1.97 \pm 0.35$ & $5.13 \pm 0.20$ & $1.57 \pm 0.76^{d}$ & $4.9 \pm 0.2(\mathrm{~A})$ & $9.7 \pm 4.7(12)$ & $3.2 \pm 1.6$ \\
\hline & $\operatorname{HNC}(1-0)^{d, f}$ & $45.2 \pm 0.1$ & $1.57 \pm 0.24$ & $1.29 \pm 0.20(\mathrm{G})$ & $3.92 \pm 0.17(\mathrm{G})$ & $3.13 \pm 1.12$ & $4.5 \pm 0.1(\mathrm{~A})$ & $1.4 \pm 0.6(13)$ & $2.3 \pm 1.0$ \\
\hline & & $13.6 \pm 0.3$ & $2.17 \pm 0.25$ & $0.22 \pm 0.20(\mathrm{G})$ & $0.92 \pm 0.24(\mathrm{G})$ & $0.27 \pm 0.04$ & $4.5 \pm 0.1(\mathrm{~A})$ & $1.6 \pm 0.4(12)$ & $0.3 \pm 0.1$ \\
\hline & & $37.1 \pm 0.3$ & $3.57 \pm 0.76$ & $0.33 \pm 0.20(\mathrm{G})$ & $1.68 \pm 0.23(\mathrm{G})$ & $0.43 \pm 0.06$ & $4.5 \pm 0.1(\mathrm{~A})$ & $3.0 \pm 0.4(12)$ & $0.5 \pm 0.1$ \\
\hline & $\operatorname{HNC}(1-0)^{e, f}$ & $44.8 \pm 0.1$ & $1.80 \pm 0.18$ & $1.93 \pm 0.30(\mathrm{G})$ & $4.74 \pm 0.16(\mathrm{G})$ & $1.87 \pm 0.91$ & $6.0 \pm 1.0$ & $1.5 \pm 0.8(13)$ & $30.6 \pm 3.1$ \\
\hline & & $36.7 \pm 0.2$ & $2.07 \pm 0.23$ & $0.36 \pm 0.30(\mathrm{G})$ & $1.21 \pm 0.35(\mathrm{G})$ & $0.47 \pm 0.10$ & $4.5 \pm 0.1(\mathrm{~A})$ & $2.1 \pm 0.6(12)$ & $0.4 \pm 0.1$ \\
\hline & $\mathrm{N}_{2} \mathrm{H}^{+}(1-0)^{d}$ & $45.1 \pm 0.1$ & $1.69 \pm 0.09$ & $1.53 \pm 0.20(\mathrm{~S})$ & $3.71 \pm 0.19(\mathrm{~S})$ & $0.77 \pm 0.04$ & $11.6 \pm 1.2$ & $1.7 \pm 0.1(13)$ & $2.8 \pm 0.3$ \\
\hline & $\mathrm{N}_{2} \mathrm{H}^{+}(1-0)^{e}$ & $45.0 \pm 0.1$ & $1.59 \pm 0.08$ & $2.07 \pm 0.24(\mathrm{~S})$ & $5.32 \pm 0.22(\mathrm{~S})$ & $3.07 \pm 0.79$ & $6.8 \pm 0.8$ & $2.5 \pm 0.7(13)$ & $5.1 \pm 1.5$ \\
\hline \multirow[t]{15}{*}{ SMM 32} & $\mathrm{H}^{13} \mathrm{CO}^{+}(1-0)$ & $51.8 \pm 0.2$ & $3.58 \pm 0.78$ & $0.45 \pm 0.17(\mathrm{G})$ & $1.81 \pm 0.19(\mathrm{G})$ & $0.72_{-0.31}^{+0.36 h}$ & $4.7 \pm 1.0$ & $6.4 \pm 3.3(12)$ & $1.1 \pm 0.6$ \\
\hline & $\mathrm{C}_{2} \mathrm{H}(1-0)^{d}$ & $52.3 \pm 0.1$ & $3.69 \pm 0.32$ & $0.58 \pm 0.18(\mathrm{~S})$ & $3.16 \pm 0.25(\mathrm{~S})$ & $3.65 \pm 1.38$ & $3.6 \pm 0.3$ & $5.8 \pm 2.2(14)$ & $98.4 \pm 38.6$ \\
\hline & $\mathrm{C}_{2} \mathrm{H}(1-0)^{e}$ & $52.0 \pm 0.4$ & $8.07 \pm 1.33$ & $0.25 \pm 0.18(\mathrm{~S})$ & $2.02 \pm 0.30(\mathrm{~S})$ & $0.56 \pm 0.10$ & $4.1 \pm 0.1(\mathrm{~A})$ & $7.8 \pm 1.2(13)$ & $63.4 \pm 11.6$ \\
\hline & $\operatorname{HNCO}\left(4_{0,4}-3_{0,3}\right)^{e}$ & $51.3 \pm 0.1$ & $2.01 \pm 0.57$ & $0.41 \pm 0.21(\mathrm{G})$ & $0.98 \pm 0.28(\mathrm{G})$ & $0.22 \pm 0.01$ & $=2 E_{\mathrm{u}} / 3=7.03$ & $5.4 \pm 1.5(12)$ & $1.3 \pm 0.4$ \\
\hline & $\operatorname{HCN}(1-0)^{d}$ & $59.4 \pm 0.1$ & $2.50 \pm 0.22$ & $0.86 \pm 0.18(\mathrm{G})$ & $2.45 \pm 0.17(\mathrm{G})$ & $1.84 \pm 0.40$ & $5.1 \pm 0.5(\mathrm{~A})$ & $3.8 \pm 0.9(13)$ & $6.5 \pm 1.7$ \\
\hline & $\operatorname{HCN}(1-0)^{e}$ & $57.3 \pm 0.6$ & $5.43 \pm 0.84$ & $0.46 \pm 0.22(\mathrm{G})$ & $2.30 \pm 0.23(\mathrm{G})$ & $0.76 \pm 0.14$ & $5.1 \pm 0.5(\mathrm{~A})$ & $3.4 \pm 0.8(13)$ & $9.3 \pm 2.4$ \\
\hline & $\mathrm{HCO}^{+}(1-0)^{d}$ & $54.3 \pm 0.1$ & $2.60 \pm 0.12$ & $1.73 \pm 0.19$ & $4.77 \pm 0.17$ & $11.80_{-5.00}^{+5.90 h}$ & $4.7 \pm 0.2$ & $7.3 \pm 3.4(13)$ & $12.4 \pm 5.9$ \\
\hline & $\mathrm{HCO}^{+}(1-0)^{e, f}$ & $53.9 \pm 0.1$ & $2.87 \pm 0.17$ & $1.40 \pm 0.18$ & $4.28 \pm 0.19$ & $1.27 \pm 0.33$ & $4.9 \pm 0.2(\mathrm{~A})$ & $9.3 \pm 2.5(12)$ & $1.7 \pm 0.5$ \\
\hline & & $13.5 \pm 0.1$ & $1.50 \pm 0.34$ & $0.42 \pm 0.18$ & $0.67 \pm 0.13$ & $0.24 \pm 0.04$ & $4.9 \pm 0.2(\mathrm{~A})$ & $8.2 \pm 1.6(11)$ & $0.1 \pm 0.03$ \\
\hline & $\mathrm{HNC}(1-0)^{d}$ & $53.0 \pm 0.1$ & $5.30 \pm 0.16$ & $0.74 \pm 0.20(\mathrm{G})$ & $4.34 \pm 0.22(\mathrm{G})$ & $1.15 \pm 0.21$ & $4.5 \pm 0.1(\mathrm{~A})$ & $1.8 \pm 0.3(13)$ & $3.1 \pm 0.6$ \\
\hline & $\operatorname{HNC}(1-0)^{e, f}$ & $52.9 \pm 0.2$ & $5.09 \pm 0.39$ & $0.60 \pm 0.19(\mathrm{G})$ & $3.32 \pm 0.23(\mathrm{G})$ & $0.87 \pm 0.14$ & $4.5 \pm 0.1(\mathrm{~A})$ & $1.3 \pm 0.2(13)$ & $6.1 \pm 1.9$ \\
\hline & & $14.1 \pm 0.3$ & $2.10 \pm 0.98$ & $0.23 \pm 0.13(\mathrm{G})$ & $0.84 \pm 0.17(\mathrm{G})$ & $0.29 \pm 0.03$ & $4.5 \pm 0.1(\mathrm{~A})$ & $1.5 \pm 0.3(12)$ & $0.7 \pm 0.2$ \\
\hline & $\mathrm{HC}_{3} \mathrm{~N}(10-9)$ & $51.6 \pm 0.1$ & $2.93 \pm 0.30$ & $0.69 \pm 0.20(\mathrm{G})$ & $2.18 \pm 0.16(\mathrm{G})$ & $0.09 \pm 0.01$ & $=E_{\mathrm{u}}=24.01$ & $9.1 \pm 0.7(12)$ & $1.5 \pm 0.2$ \\
\hline & $\mathrm{N}_{2} \mathrm{H}^{+}(1-0)^{d}$ & $51.4 \pm 0.1$ & $2.87 \pm 0.11$ & $1.82 \pm 0.24(\mathrm{~S})$ & $7.21 \pm 0.22(\mathrm{~S})$ & $1.86 \pm 0.53$ & $7.9 \pm 1.3$ & $3.5 \pm 1.0(13)$ & $5.9 \pm 1.8$ \\
\hline & $\mathrm{N}_{2} \mathrm{H}^{+}(1-0)^{e}$ & $51.5 \pm 0.1$ & $2.76 \pm 0.08$ & $2.10 \pm 0.22(\mathrm{~S})$ & $8.96 \pm 0.23(\mathrm{~S})$ & $3.20 \pm 0.47$ & $6.8 \pm 0.5$ & $4.6 \pm 0.7(13)$ & $7.7 \pm 1.4$ \\
\hline
\end{tabular}




\section{Appendix A: Kinematic distances and physical properties of the clumps}

The kinematic distances of the clumps listed in Col. (4) of Table 1 are based on the Galactic rotation curve model by Reid et al. (2009). As the sources are associated with IRDCs, it is assumed that they lie at a near distance in which case there is more IR background radiation against which to see the source in absorption. The clump distances were adopted from Paper I with the following exception. In Paper I, for the first quadrant field G1.87 we had radial velocity data only towards a filamentary cloud near the field centre (see Figs. 1 and 5 in Paper I). As discussed in Paper I, the negative radial velocity of the cloud $\left(\sim-41 \mathrm{~km} \mathrm{~s}^{-1}\right)$ suggests that it lies at a far distance. The far distance solution $(\sim 10.6 \mathrm{kpc})$ was therefore adopted for all the clumps in the field. In the present paper, we used the MALT90 $\mathrm{N}_{2} \mathrm{H}^{+}(1-0)$ radial velocity data to determine the G1.87 clump distances. As a high-density gas tracer, $\mathrm{N}_{2} \mathrm{H}^{+}$is expected to be well-suited for this purpose. Moreover, $\mathrm{N}_{2} \mathrm{H}^{+}(1-0)$ was detected towards all sources in the present study. The radial velocities of the clumps were typically found to be $\sim 40-50 \mathrm{~km} \mathrm{~s}^{-1}$ (i.e. positive rather than negative), and the corresponding near kinematic distances were derived to be $\sim 7 \mathrm{kpc}$ as shown in Table 1 . We note that the near-far kinematic distance ambiguity towards G13.22-SMM 29 (IRAS 18117-1738) was resolved by Sewilo et al. (2004). This source was placed at the far distance because $\mathrm{H}_{2} \mathrm{CO}$ absorption was seen between the source velocity and the velocity at the tangent point.
The clump effective radii listed in Table 1 correspond to the kinematic distances explained above. The rest of the physical properties of the clumps listed in Table 1 were revised from those presented in Paper I by making the following modifications. The masses and densities of the clumps were previously calculated by assuming that the dust-to-gas mass ratio is $1 / 100$. However, this value refers to the canonical dust-to-hydrogen mass ratio, $M_{\text {dust }} / M_{\mathrm{H}}$ (e.g. Draine 2011; Table 23.1 therein). Assuming that the clumps' chemical composition is similar to the solar mixture, i.e. the mass fractions for hydrogen, helium, and heavier elements are $X=0.71, Y=0.27$, and $Z=0.02$, respectively, the ratio of total mass $(\mathrm{H}+\mathrm{He}+$ metals $)$ to hydrogen mass is $1 / X \simeq 1.41$. The total dust-to-gas mass ratio is therefore $M_{\text {dust }} / M_{\text {gas }}=M_{\text {dust }} /\left(1.41 M_{\mathrm{H}}\right)=1 / 141$. For the assumed gas composition, the mean molecular weight per $\mathrm{H}_{2}$ molecule, needed in the calculation of the column and number densities, is $\mu_{\mathrm{H}_{2}} \simeq 2.82$ (Kauffmann et al. 2008; Appendix A.1 therein). As explained in Paper I, the dust temperature was assumed to be $T_{\text {dust }}=15 \mathrm{~K}$ for IR-dark clumps, and $20 \mathrm{~K}$ for clumps associated with Spitzer IR emission. For G2.11-SMM 5 and G13.22-SMM 29, which are associated with IRAS sources, the dust colour temperatures were derived to be 30 and $18.9 \mathrm{~K}$, respectively (Paper I). For G13.22-SMM 32, which is associated with an H II region, we adopted the value ${ }^{10} T_{\text {dust }}=30 \mathrm{~K}$. Finally, the dust opacity per unit dust mass at $870 \mu \mathrm{m}$ was taken to be $\kappa_{870}=1.38 \mathrm{~cm}^{2} \mathrm{~g}^{-1}$, the value interpolated from the widely used Ossenkopf \& Henning (1994) model describing graphite-silicate dust grains that have coagulated and accreted thin ice mantles over a period of $10^{5} \mathrm{yr}$ at a gas density of $10^{5} \mathrm{~cm}^{-3}$.

\footnotetext{
$\overline{10}$ Recently, Hoq et al. (2013) showed that the median dust temperature of the MALT90 clumps increases as a function of the stage of evolution (their Fig. 2). For quiescent (IR-dark) clumps, clumps containing YSOs, and $\mathrm{HII} / \mathrm{PDR}$ sources the median dust temperatures were determined to be $13.9,17.9$, and $26.0 \mathrm{~K}$, respectively. These are comparable to the values we have adopted.
} 


\section{Appendix B: Maps of spectral-line emission}

The integrated intensity maps of the detected spectral lines are presented in Figs. B.1-B.14. In each panel, the line emission is shown as contours overlaid on the Spitzer $8 \mu \mathrm{m}$ image.
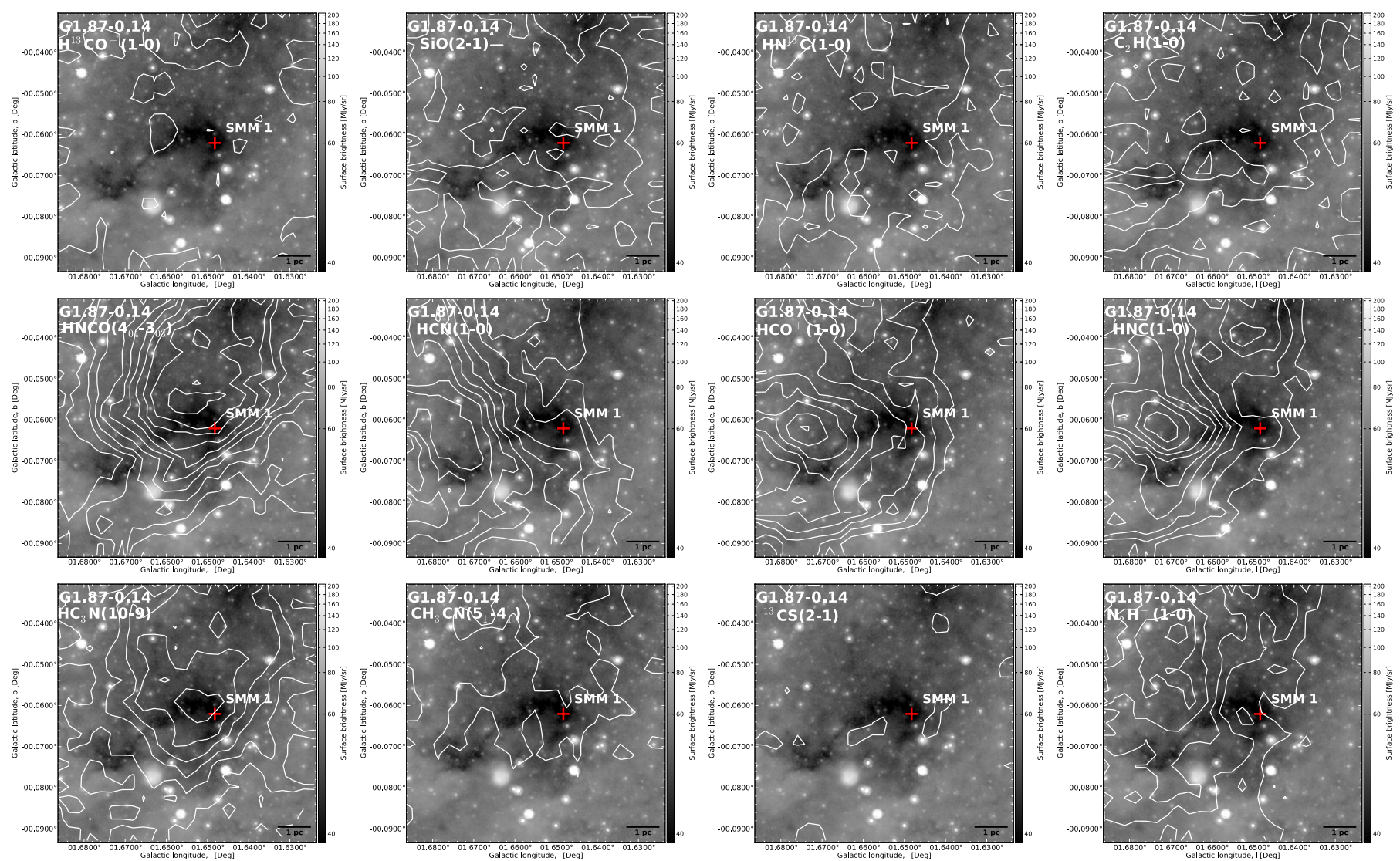

Fig. B.1. Contour maps of integrated intensity of the MALT90 lines detected towards G1.87-SMM 1. In each panel, the contours are overlaid on the arcsinh-scaled Spitzer $8 \mu \mathrm{m}$ image (cf. Fig. 1). The contour levels start at $3 \sigma$ for $\mathrm{H}^{13} \mathrm{CO}^{+}, \mathrm{SiO}, \mathrm{HN}^{13} \mathrm{C}, \mathrm{C}_{2} \mathrm{H}, \mathrm{CH}_{3} \mathrm{CN}$, and ${ }^{13} \mathrm{CS}$. For $\mathrm{HNCO}\left(4_{0,4}-3_{0,3}\right), \mathrm{HCN}, \mathrm{HCO}^{+}, \mathrm{HNC}, \mathrm{HC}_{3} \mathrm{~N}$, and $\mathrm{N}_{2} \mathrm{H}^{+}$, the contours start at $26 \sigma, 23 \sigma, 20 \sigma, 30 \sigma, 6 \sigma$, and $15 \sigma$, respectively. In all cases, the contours go in steps of $3 \sigma$. The average $1 \sigma$ value in $T_{\mathrm{MB}}$ units is $\sim 0.69 \mathrm{~K} \mathrm{~km} \mathrm{~s}^{-1}$. The red plus sign marks the LABOCA $870 \mu \mathrm{m}$ peak position of the clump. A scale bar indicating the $1 \mathrm{pc}$ projected length is indicated. The line emission is extended in many cases, and the $\mathrm{HCN}, \mathrm{HCO}^{+}$, and $\mathrm{HNC}$ emissions are well correlated with each other. The $\mathrm{N}_{2} \mathrm{H}^{+}$emission also shows some resemblance to these species. The spatial distributions of $\mathrm{HNCO}$ and $\mathrm{HC}_{3} \mathrm{~N}$ appear to be similar to each other, while weak $\mathrm{CH}_{3} \mathrm{CN}$ emission traces reasonably well the $8 \mu$ m absorption feature. 
O. Miettinen: A MALT90 chemical study of clumps within IRDCs
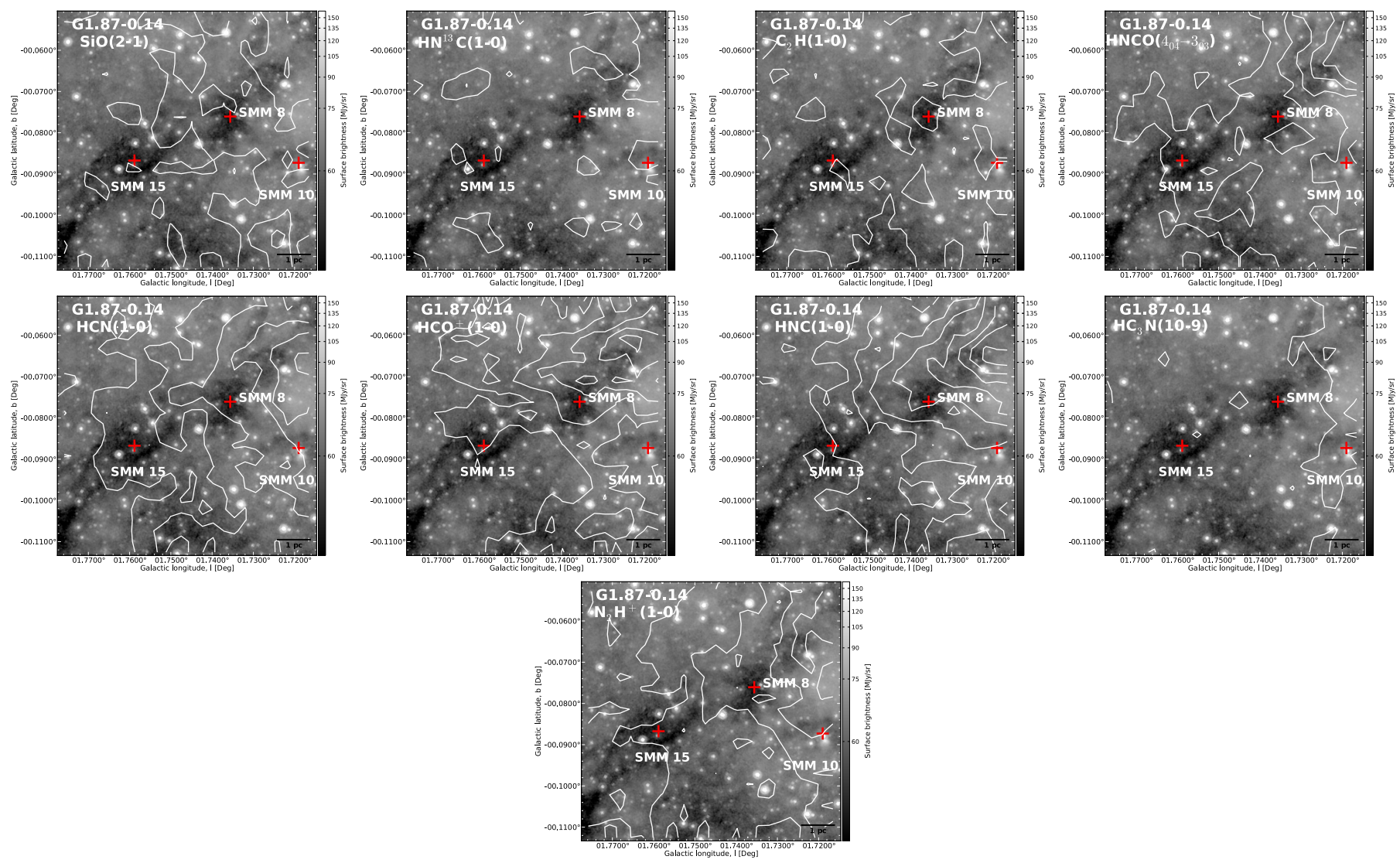

Fig. B.2. Similar to Fig. B.1 but towards G1.87-SMM 8, 10, 15. The contour levels start at $3 \sigma$ for $\mathrm{SiO}, \mathrm{HN}^{13} \mathrm{C}, \mathrm{C}_{2} \mathrm{H}$, and $\mathrm{HC}_{3} \mathrm{~N}$. For $\mathrm{HNCO}\left(4_{0,4}-3_{0,3}\right), \mathrm{HCN}, \mathrm{HCO}^{+}, \mathrm{HNC}$, and $\mathrm{N}_{2} \mathrm{H}^{+}$, the contours start at $9 \sigma, 16 \sigma, 15 \sigma, 12 \sigma$, and $5 \sigma$, respectively. In all cases, the contours go in steps of $3 \sigma$. The average $1 \sigma$ value in $T_{\mathrm{MB}}$ units is $\sim 0.70 \mathrm{~K} \mathrm{~km} \mathrm{~s}^{-1}$. The LABOCA $870 \mu \mathrm{m}$ peak positions of the clumps are marked by red plus

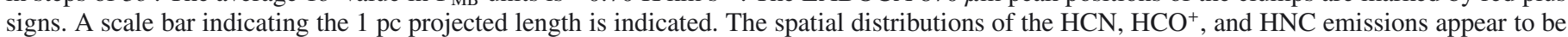
quite similar. Those of $\mathrm{HNCO}$ and $\mathrm{N}_{2} \mathrm{H}^{+}$show some similarities also. 
A\&A 562, A3 (2014)

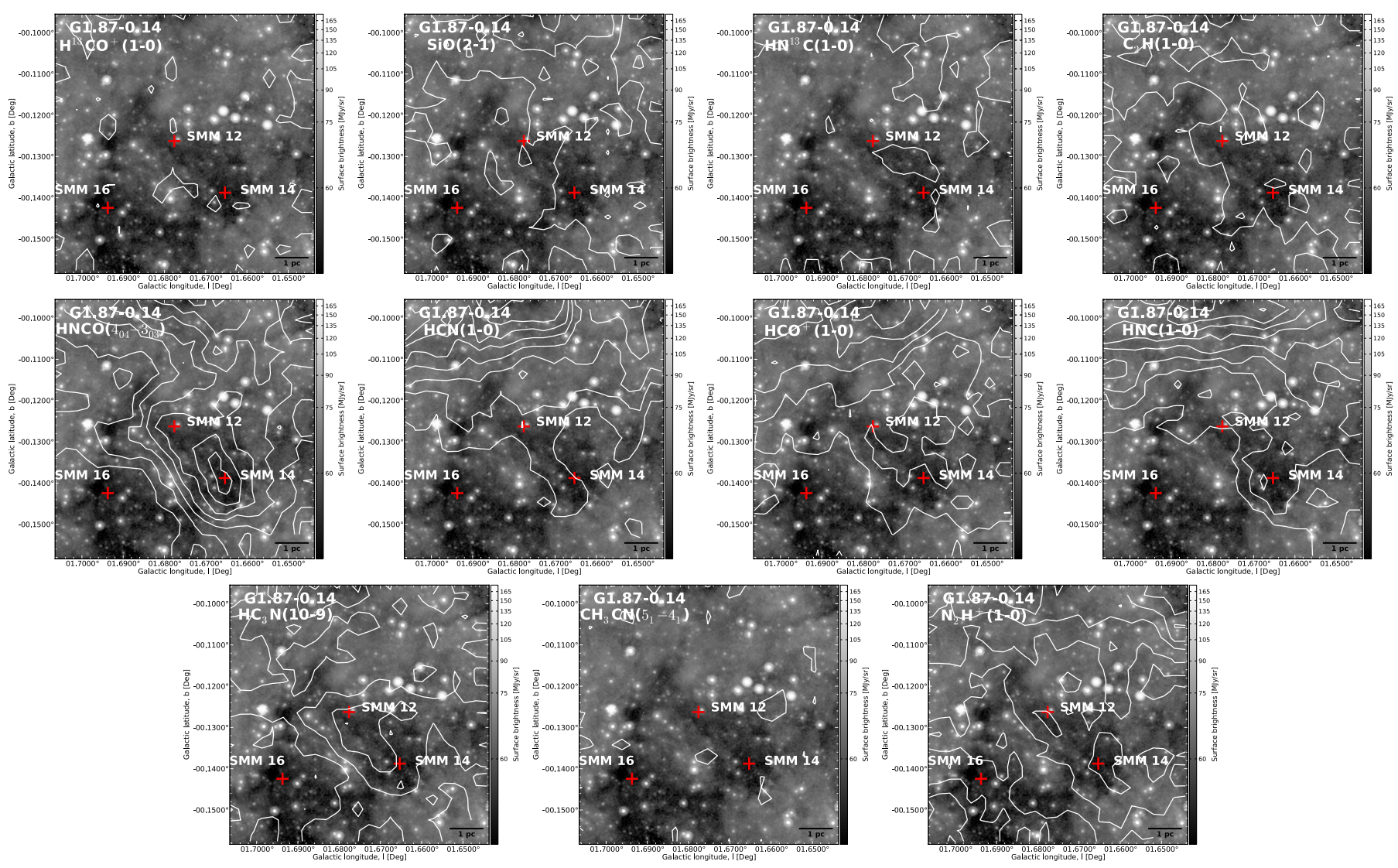

Fig. B.3. Similar to Fig. B.1 but towards G1.87-SMM 12, 14, 16. The contour levels start at $3 \sigma$ for $\mathrm{H}^{13} \mathrm{CO}^{+}, \mathrm{HN}^{13} \mathrm{C}_{2} \mathrm{C}_{2} \mathrm{H}, \mathrm{HC}_{3} \mathrm{~N}$, and $\mathrm{CH}_{3} \mathrm{CN}$. For $\mathrm{SiO}, \mathrm{HNCO}\left(4_{0,4}-3_{0,3}\right), \mathrm{HCN}_{1} \mathrm{HCO}^{+}, \mathrm{HNC}$, and $\mathrm{N}_{2} \mathrm{H}^{+}$, the contours start at $5 \sigma, 15 \sigma, 19 \sigma, 10 \sigma, 15 \sigma$, and $6 \sigma$, respectively. In all cases, the contours go in steps of $3 \sigma$. The average $1 \sigma$ value in $T_{\mathrm{MB}}$ units is $\sim 0.63 \mathrm{~K} \mathrm{~km} \mathrm{~s}^{-1}$. The LABOCA $870 \mu \mathrm{m}$ peak positions of the clumps are marked by red plus signs. A scale bar indicating the 1 pc projected length is indicated. The emissions of $\mathrm{HNCO}, \mathrm{HCN}, \mathrm{HCO}^{+}, \mathrm{HNC}, \mathrm{HC}_{3} \mathrm{~N}$, and $\mathrm{N}_{2} \mathrm{H}^{+}$ are extended in a similar fashion, but the HNCO emission is clearly the strongest. 
O. Miettinen: A MALT90 chemical study of clumps within IRDCs
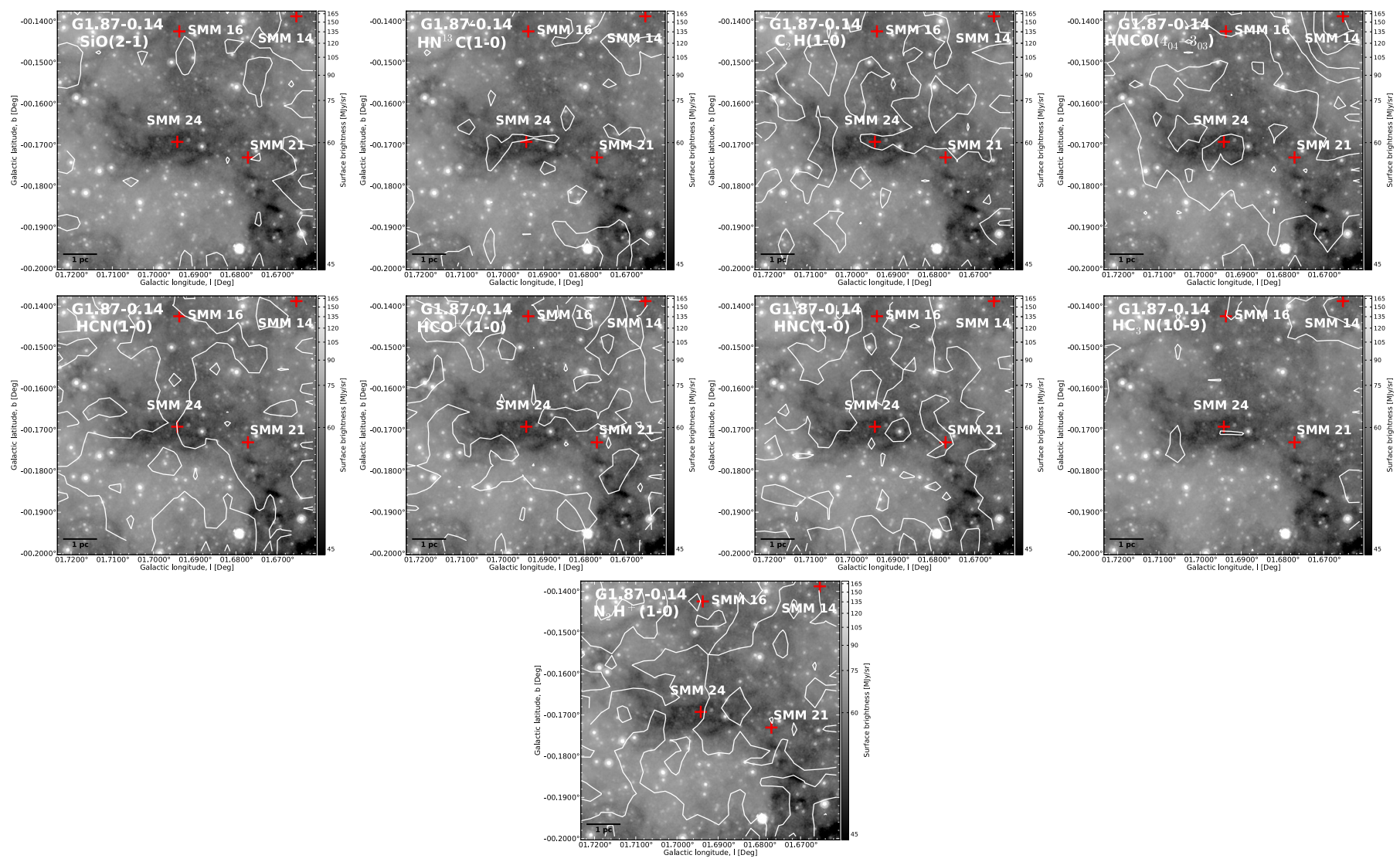

Fig. B.4. Similar to Fig. B.1 but towards G1.87-SMM 14, 16, 17, 21, 24. The contour levels start at $3 \sigma$ for $\mathrm{SiO}, \mathrm{HN}^{13} \mathrm{C}, \mathrm{C}_{2} \mathrm{H}$, and $\mathrm{HC}_{3} \mathrm{~N}$. For $\mathrm{HNCO}\left(4_{0,4}-3_{0,3}\right), \mathrm{HCN}, \mathrm{HCO}^{+}, \mathrm{HNC}$, and $\mathrm{N}_{2} \mathrm{H}^{+}$, the contours start at $8 \sigma, 8 \sigma, 5 \sigma, 5 \sigma$, and $5 \sigma$, respectively. In all cases, the contours go in steps of $3 \sigma$. The average $1 \sigma$ value in $T_{\mathrm{MB}}$ units is $\sim 0.78 \mathrm{~K} \mathrm{~km} \mathrm{~s}^{-1}$. The LABOCA $870 \mu \mathrm{m}$ peak positions of the clumps are marked by red plus signs. The $870 \mu \mathrm{m}$ peak of SMM 17 (west of SMM 21) lies just outside the MALT90 map (cf. Fig. 1, middle left panel). A scale bar indicating the 1 pc projected length is indicated. The HCN and HNC emissions show some morphological similarities. 

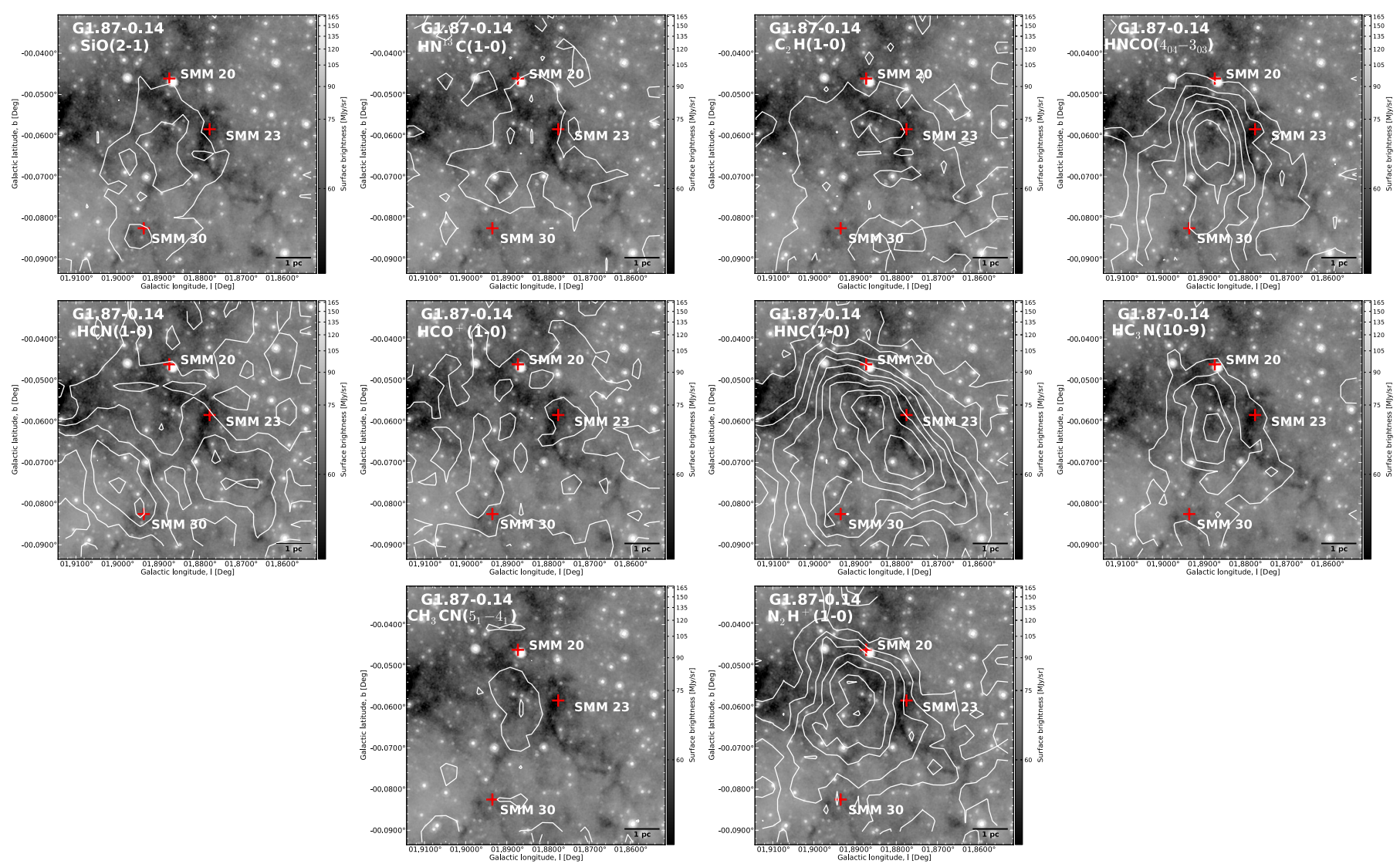

Fig. B.5. Similar to Fig. B.1 but towards G1.87-SMM 20, 23, 30. The contour levels start at $3 \sigma$ for $\mathrm{HN}^{13} \mathrm{C}, \mathrm{C}_{2} \mathrm{H}$, and $\mathrm{CH}_{3} \mathrm{CN}$, and $5 \sigma$ for $\mathrm{SiO}$, $\mathrm{HNCO}\left(4_{0,4}-3_{0,3}\right), \mathrm{HCN}, \mathrm{HCO}^{+}, \mathrm{HNC}, \mathrm{HC}_{3} \mathrm{~N}$, and $\mathrm{N}_{2} \mathrm{H}^{+}$. In all cases, the contours go in steps of $3 \sigma$. The average $1 \sigma$ value in $T_{\mathrm{MB}}$ units is $\sim 0.63 \mathrm{~K} \mathrm{~km} \mathrm{~s}^{-1}$. The LABOCA $870 \mu \mathrm{m}$ peak positions of the clumps are marked by red plus signs. A scale bar indicating the $1 \mathrm{pc}$ projected length is indicated. Good correlations are seen between the peak emissions of $\mathrm{SiO}, \mathrm{HNCO}, \mathrm{HC}_{3} \mathrm{~N}, \mathrm{CH}_{3} \mathrm{CN}$, and $\mathrm{N}_{2} \mathrm{H}^{+}$. The $\mathrm{HNC}$ emission also shows its maxima in-between the submm peaks. 
O. Miettinen: A MALT90 chemical study of clumps within IRDCs
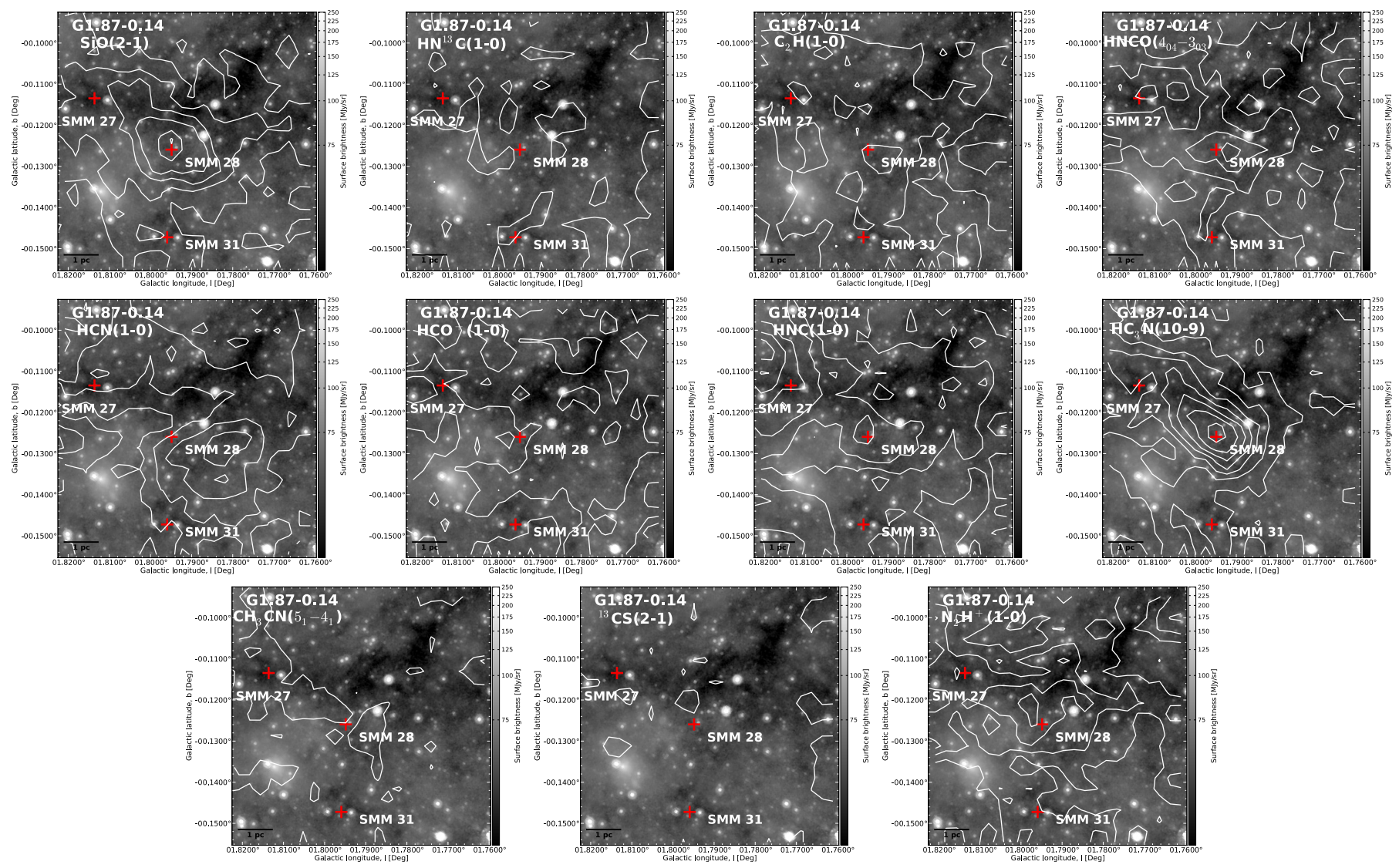

Fig. B.6. Similar to Fig. B.1 but towards G1.87-SMM 27, 28, 31. The contour levels start at $3 \sigma$ for $\mathrm{SiO}, \mathrm{HN}^{13} \mathrm{C}, \mathrm{C}_{2} \mathrm{H}, \mathrm{CH}_{3} \mathrm{CN}$, and ${ }^{13} \mathrm{CS}$. For, $\mathrm{HNCO}\left(4_{0,4}-3_{0,3}\right), \mathrm{HNC}, \mathrm{HCN}, \mathrm{HCO}^{+}, \mathrm{HC}_{3} \mathrm{~N}$, and $\mathrm{N}_{2} \mathrm{H}^{+}$, the contours start at $9 \sigma, 7 \sigma, 7 \sigma, 4 \sigma, 4 \sigma$, and $5 \sigma$, respectively. In all cases, the contours go in steps of $3 \sigma$. The average $1 \sigma$ value in $T_{\mathrm{MB}}$ units is $\sim 0.63 \mathrm{~K} \mathrm{~km} \mathrm{~s}^{-1}$. The LABOCA $870 \mu \mathrm{m}$ peak positions are marked by red plus signs. A scale bar indicating the 1 pc projected length is indicated. The $\mathrm{SiO}$ and $\mathrm{HC}_{3} \mathrm{~N}$ emission morphologies resemble each other. $\mathrm{HNC}$ and $\mathrm{N}_{2} \mathrm{H}^{+}$also share some common features (e.g. peak close to SMM 28).
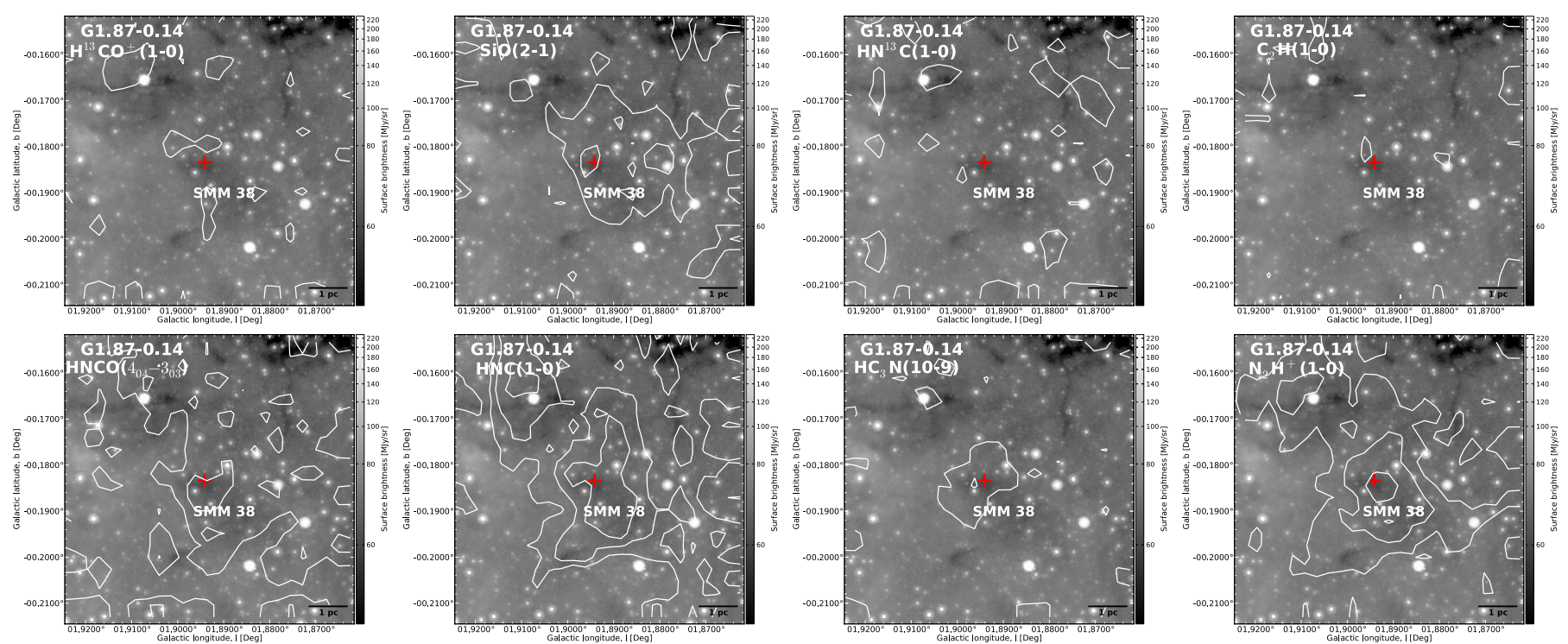

Fig. B.7. Similar to Fig. B.1 but towards G1.87-SMM 38. The contour levels start at $3 \sigma$ for $\mathrm{H}^{13} \mathrm{CO}^{+}, \mathrm{SiO}_{,} \mathrm{HN}^{13} \mathrm{C}, \mathrm{C}_{2} \mathrm{H}, \mathrm{HNCO}\left(4_{0,4}-3_{0,3}\right), \mathrm{HC}_{3} \mathrm{~N}$, and $\mathrm{N}_{2} \mathrm{H}^{+}$. For $\mathrm{HNC}$, the contours start at $5 \sigma$. In all cases, the contours go in steps of $3 \sigma$. The average $1 \sigma$ value in $T_{\mathrm{MB}}$ units is $\sim 0.77 \mathrm{~K} \mathrm{~km} \mathrm{~s}{ }^{-1}$. The clump's LABOCA $870 \mu \mathrm{m}$ peak position is marked by a red plus sign. A scale bar indicating the $1 \mathrm{pc}$ projected length is indicated. We note that the $\mathrm{SiO}, \mathrm{HNC}, \mathrm{HC}_{3} \mathrm{~N}$, and $\mathrm{N}_{2} \mathrm{H}^{+}$emissions peak towards the submm maximum. $\mathrm{HNC}$ and $\mathrm{N}_{2} \mathrm{H}^{+}$show otherwise comparable spatial distributions. 

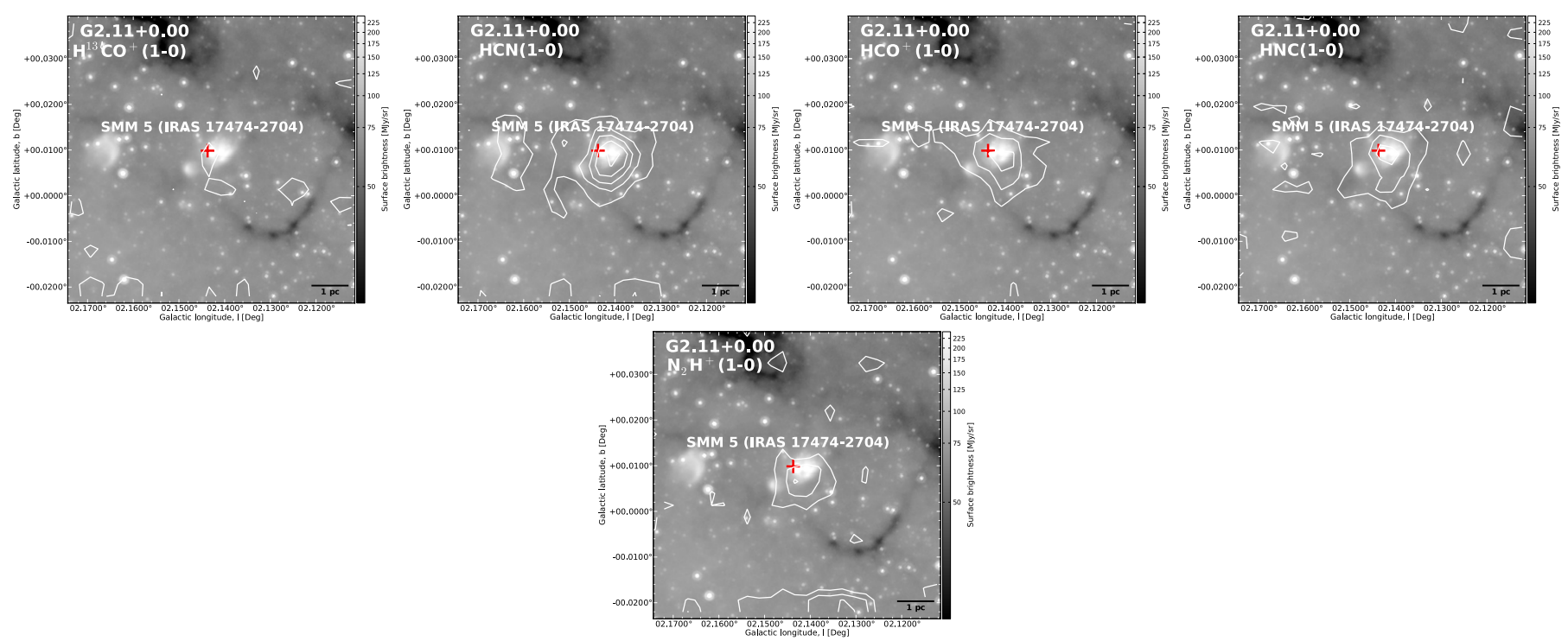

Fig. B.8. Similar to Fig. B.1 but towards G2.11-SMM 5. In each panel, the contour levels start at $3 \sigma$, and go in steps of $3 \sigma$. The average $1 \sigma$ value in $T_{\mathrm{MB}}$ units is $\sim 0.68 \mathrm{~K} \mathrm{~km} \mathrm{~s}^{-1}$. The clump's LABOCA $870 \mu \mathrm{m}$ peak position is marked by a red plus sign. A scale bar indicating the $1 \mathrm{pc}$ projected length is indicated. The $\mathrm{HCN}, \mathrm{HCO}^{+}, \mathrm{HNC}$, and $\mathrm{N}_{2} \mathrm{H}^{+}$show similar clump-like morphologies. The first two species also show an extension to the west of the emission peak.
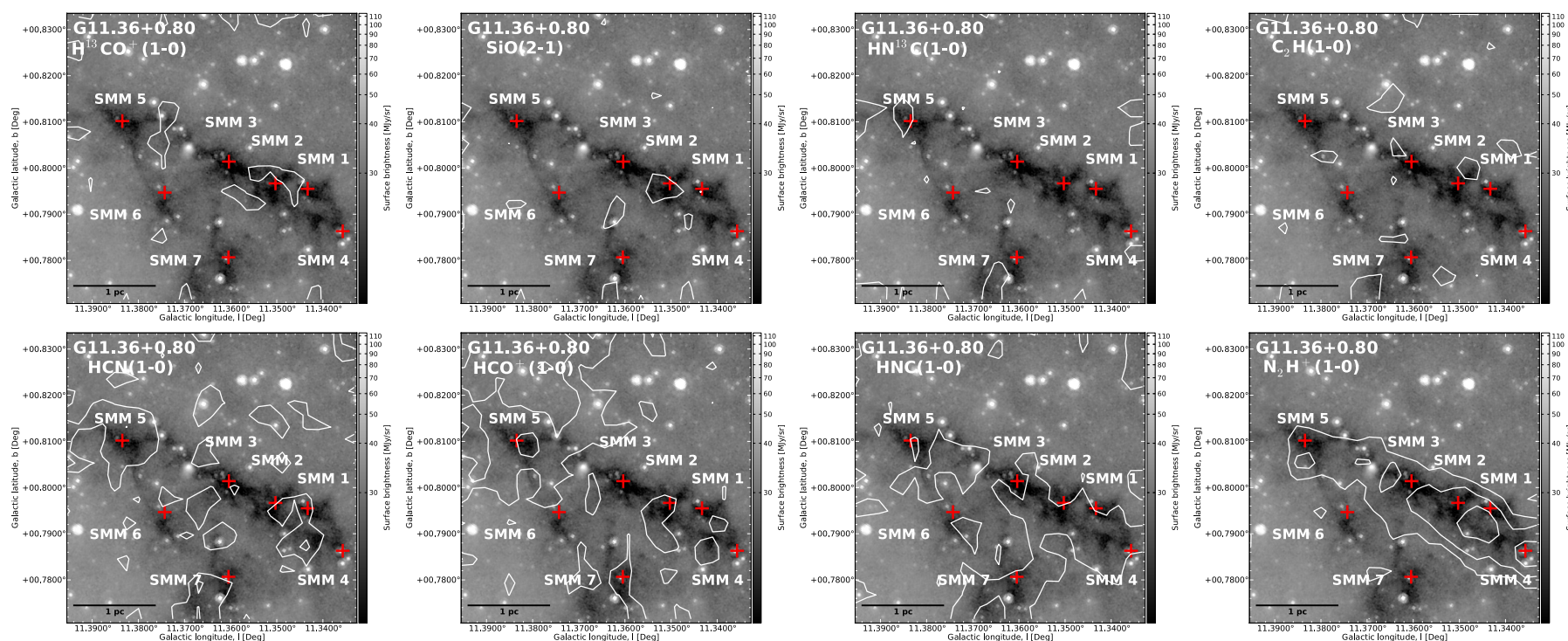

Fig. B.9. Similar to Fig. B.1 but towards the filamentary IRDC G11.36. The contour levels start at $3 \sigma$ in all cases except for $\mathrm{N}_{2} \mathrm{H}^{+}$, where they start at $5 \sigma$. In all cases, the contours go in steps of $3 \sigma$. The average $1 \sigma$ value in $T_{\mathrm{MB}}$ units is $\sim 0.74 \mathrm{~K} \mathrm{~km} \mathrm{~s}^{-1}$. The LABOCA $870 \mu \mathrm{m}$ peak positions of the clumps are marked by red plus signs. A scale bar indicating the $1 \mathrm{pc}$ projected length is indicated. The $\mathrm{HCN}$ and $\mathrm{HCO}^{+}$appear to concentrate towards SMM 5, while $\mathrm{HNC}$, and particularly $\mathrm{N}_{2} \mathrm{H}^{+}$, trace the filamentary $8 \mu \mathrm{m}$ absorption feature. 
O. Miettinen: A MALT90 chemical study of clumps within IRDCs
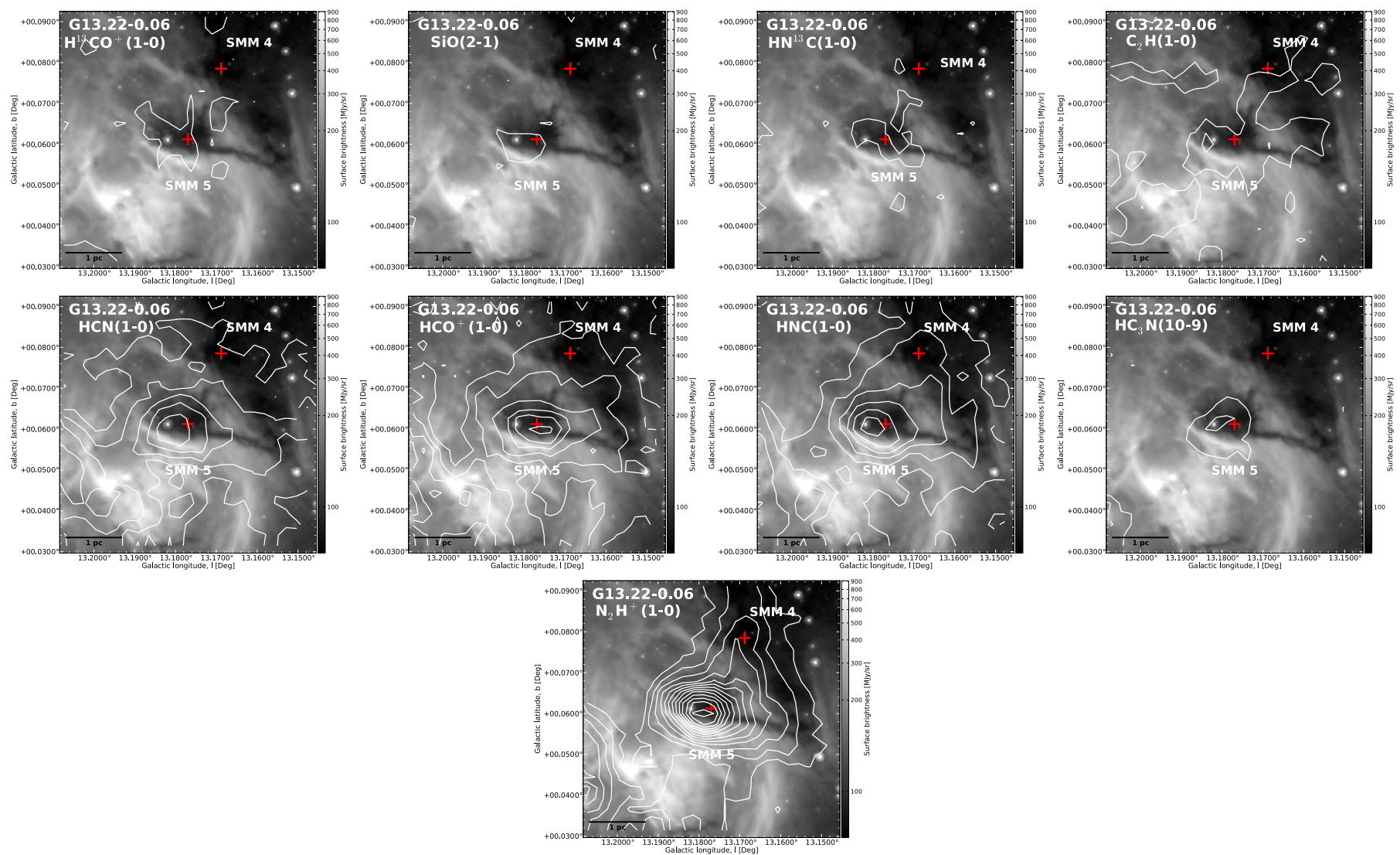

Fig. B.10. Similar to Fig. B.1 but towards G13.22-SMM 4, 5 around the N10/11 IR-bubble pair. The contour levels start at $3 \sigma$ for $\mathrm{H}^{13} \mathrm{CO}^{+}, \mathrm{SiO}_{\text {, }}$ $\mathrm{HN}^{13} \mathrm{C}, \mathrm{C}_{2} \mathrm{H}$, and $\mathrm{HC}_{3} \mathrm{~N}$, while for $\mathrm{HCN}, \mathrm{HCO}^{+}, \mathrm{HNC}$, and $\mathrm{N}_{2} \mathrm{H}^{+}$they start at $5 \sigma$. In all cases, the contours go in steps of $3 \sigma$. The average $1 \sigma$ value in $T_{\mathrm{MB}}$ units is $\sim 0.79 \mathrm{~K} \mathrm{~km} \mathrm{~s}^{-1}$. The LABOCA $870 \mu \mathrm{m}$ peak positions of the clumps are marked by red plus signs. A scale bar indicating the $1 \mathrm{pc}$ projected length is indicated. We note the extended morphological similarities between $\mathrm{HCN}, \mathrm{HCO}^{+}, \mathrm{HNC}$ and $\mathrm{N}_{2} \mathrm{H}^{+}$. $\mathrm{C}_{2} \mathrm{H}$ shows a ridge-like emission along the northwest-southeast direction. The emission of all the species peaks towards SMM 5.
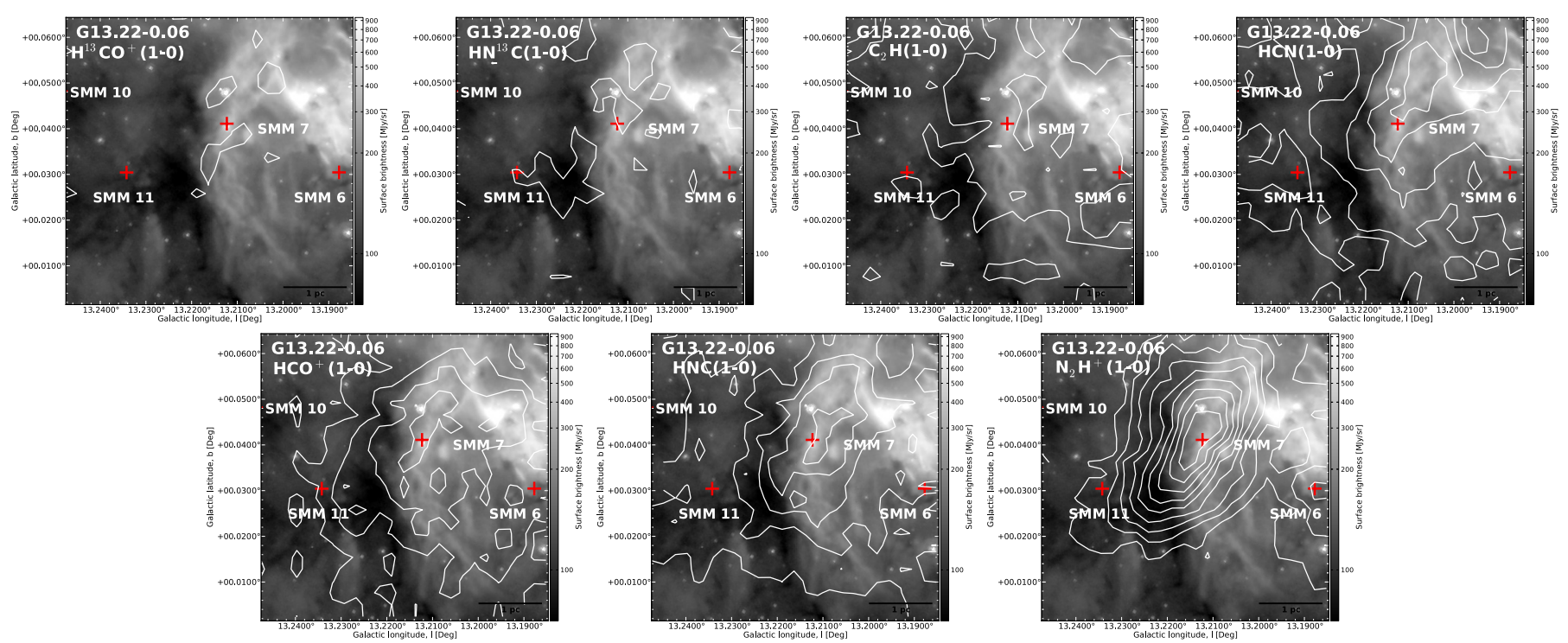

Fig. B.11. Similar to Fig. B.1 but towards G13.22-SMM 6, 7, 10, 11 around the N10/11 bubble. The contour levels start at $3 \sigma$ for $\mathrm{H}^{13} \mathrm{CO}^{+}, \mathrm{HN}^{13} \mathrm{C}$, and $\mathrm{C}_{2} \mathrm{H}$, while for $\mathrm{HCN}, \mathrm{HCO}^{+}$, $\mathrm{HNC}$, and $\mathrm{N}_{2} \mathrm{H}^{+}$they start at $6 \sigma, 6 \sigma, 5 \sigma$, and $6 \sigma$, respectively. In all cases, the contours go in steps of $3 \sigma$. The average $1 \sigma$ value in $T_{\mathrm{MB}}$ units is $\sim 0.70 \mathrm{~K} \mathrm{~km} \mathrm{~s}^{-1}$. The LABOCA $870 \mu \mathrm{m}$ peak positions of the clumps are marked by red plus signs. The $870 \mu \mathrm{m}$ peak of SMM 10 lies just outside the MALT90 map boundary. A scale bar indicating the 1 pc projected length is indicated. Emission from $\mathrm{C}_{2} \mathrm{H}$, $\mathrm{HCN}, \mathrm{HCO}^{+}, \mathrm{HNC}$, and $\mathrm{N}_{2} \mathrm{H}^{+}$are similarly spatially extended, peaking towards SMM 7 . 

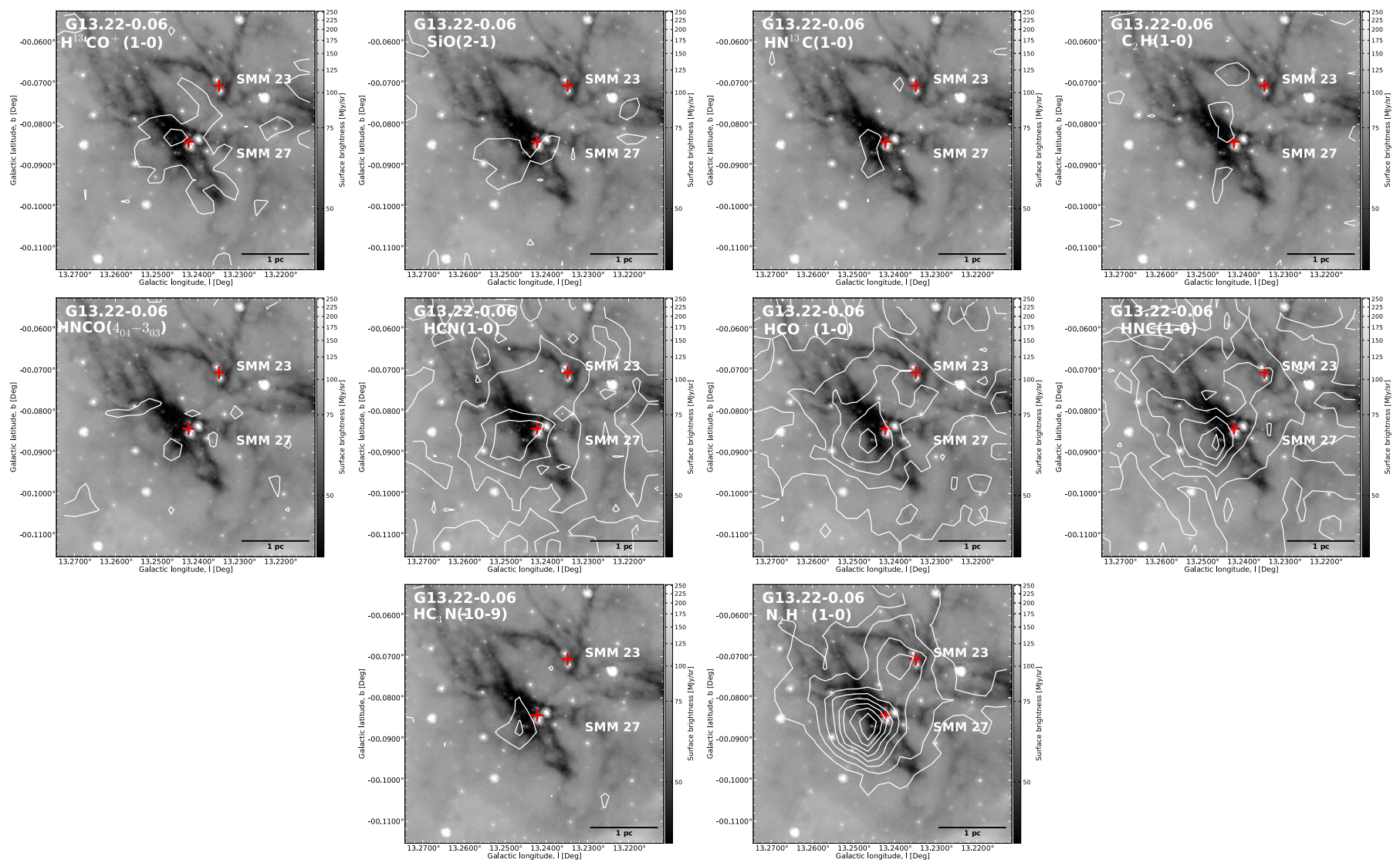

Fig. B.12. Similar to Fig. B.1 but towards G13.22-SMM 23, 27. The contour levels start at $3 \sigma$ for $\mathrm{H}^{13} \mathrm{CO}^{+}, \mathrm{SiO}, \mathrm{HN}{ }^{13} \mathrm{C}, \mathrm{C}_{2} \mathrm{H}, \mathrm{HNCO}\left(4_{0,4}-3_{0,3}\right)$, $\mathrm{HCN}$, and $\mathrm{HC}_{3} \mathrm{~N}$, while for $\mathrm{HCO}^{+}, \mathrm{HNC}$, and $\mathrm{N}_{2} \mathrm{H}^{+}$they start at $5 \sigma, 4 \sigma$, and $5 \sigma$, respectively. In all cases, the contours go in steps of $3 \sigma$. The average $1 \sigma$ value in $T_{\mathrm{MB}}$ units is $\sim 0.69 \mathrm{~K} \mathrm{~km} \mathrm{~s}^{-1}$. The LABOCA $870 \mu \mathrm{m}$ peak positions of the clumps are marked by red plus signs. A scale bar indicating the $1 \mathrm{pc}$ projected length is indicated. $\mathrm{HCN}, \mathrm{HCO}^{+}, \mathrm{HNC}$, and $\mathrm{N}_{2} \mathrm{H}^{+}$show similar spatial distributions and their emission peaks are well correlated. Although weaker, $\mathrm{H}^{13} \mathrm{CO}^{+}$and $\mathrm{HC}_{3} \mathrm{~N}$ also peak near $\mathrm{SMM} 27$.
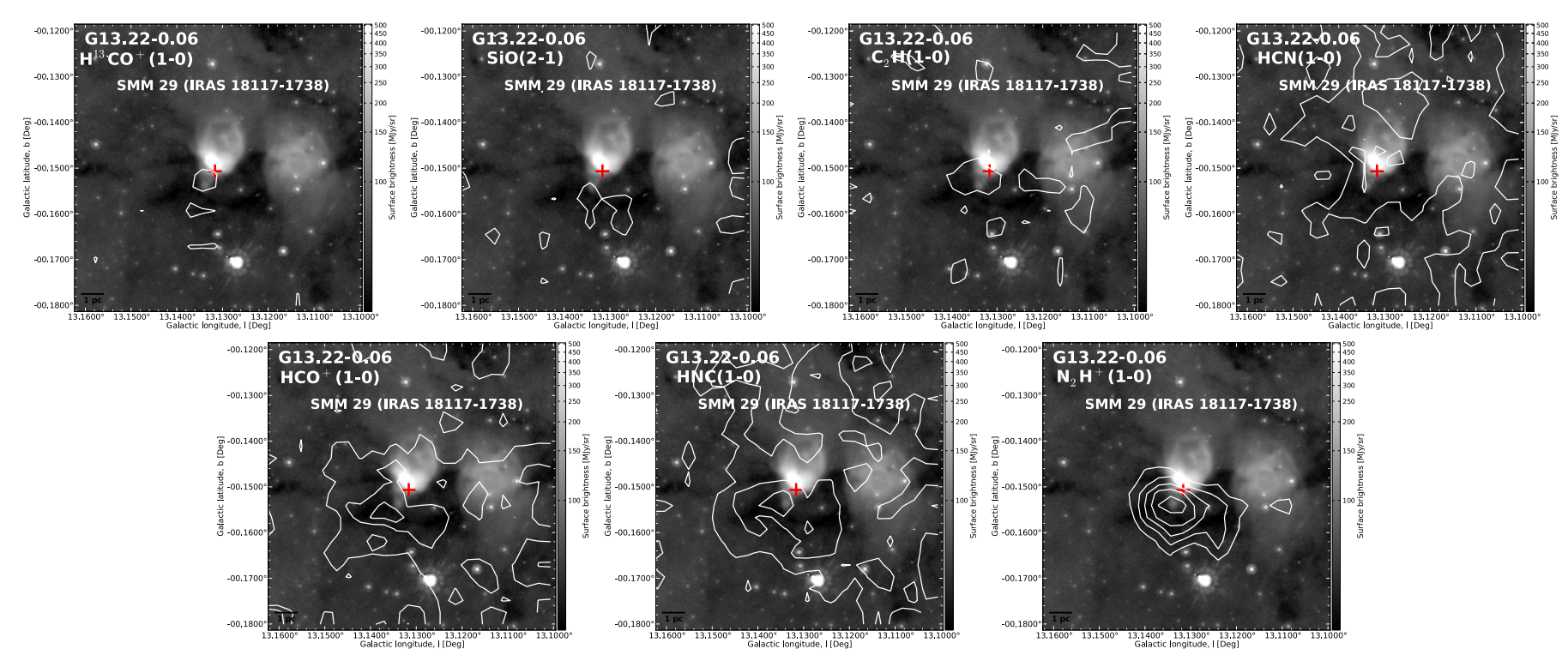

Fig. B.13. Similar to Fig. B.1 but towards G13.22-SMM 29. The contour levels start at $3 \sigma$ for $\mathrm{H}^{13} \mathrm{CO}^{+}, \mathrm{SiO}$, and $\mathrm{C}_{2} \mathrm{H}$, at $4 \sigma$ for $\mathrm{HCN}$ and $\mathrm{HNC}$, and at $5 \sigma$ for $\mathrm{HCO}^{+}$and $\mathrm{N}_{2} \mathrm{H}^{+}$. In all cases, the contours go in steps of $3 \sigma$. The average $1 \sigma$ value in $T_{\mathrm{MB}}$ units is $\sim 0.66 \mathrm{~K} \mathrm{~km} \mathrm{~s}{ }^{-1}$. The LABOCA $870 \mu \mathrm{m}$ peak position of the clump is marked by a red plus signs. A scale bar indicating the $1 \mathrm{pc}$ projected length is indicated. The $\mathrm{HCO}^{+}$and HNC emissions show similar distributions, with $\mathrm{HCN}$ sharing some spatial features. The strong $\mathrm{N}_{2} \mathrm{H}^{+}$emission peaks towards the $\mathrm{HNC}$ maximum. 

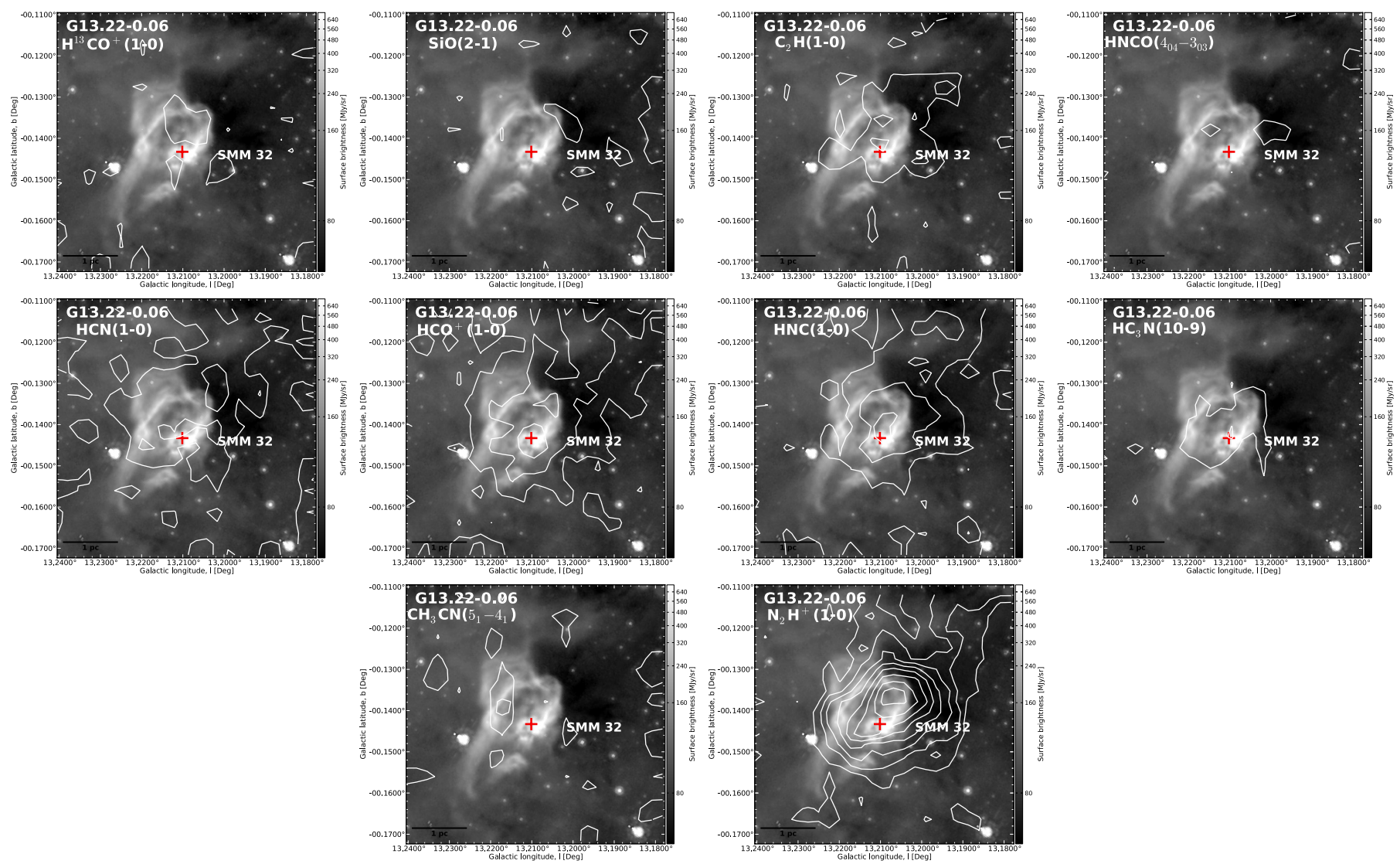

Fig. B.14. Similar to Fig. B.1 but towards G13.22-SMM 32. The contour levels start at $3 \sigma$ for $\mathrm{H}^{13} \mathrm{CO}^{+}, \mathrm{SiO}, \mathrm{C}_{2} \mathrm{H}, \mathrm{HNCO}\left(4_{0,4}-3_{0,3}\right), \mathrm{HCN}$, $\mathrm{HC}_{3} \mathrm{~N}$, and $\mathrm{CH}_{3} \mathrm{CN}$, while for $\mathrm{HCO}^{+}, \mathrm{HNC}$, and $\mathrm{N}_{2} \mathrm{H}^{+}$they start at $4 \sigma, 5 \sigma$, and $4 \sigma$, respectively. In all cases, the contours go in steps of $3 \sigma$. The average $1 \sigma$ value in $T_{\mathrm{MB}}$ units is $\sim 0.57 \mathrm{~K} \mathrm{~km} \mathrm{~s}^{-1}$. The LABOCA $870 \mu \mathrm{m}$ peak position of the clump is marked by a red plus sign. A scale bar indicating the $1 \mathrm{pc}$ projected length is indicated. The spatial distributions of $\mathrm{HCN}, \mathrm{HCO}^{+}, \mathrm{HNC}$, and $\mathrm{N}_{2} \mathrm{H}^{+}$are quite similar, but the different peak positions of $\mathrm{HCO}^{+}$and $\mathrm{N}_{2} \mathrm{H}^{+}$should be noted (as expected from their chemistry). $\mathrm{C}_{2} \mathrm{H}$ and $\mathrm{HC}_{3} \mathrm{~N}$ also show weaker emission towards $\mathrm{SMM} 32$, with some morphological similarities to the strongly emitting species. 


\section{Appendix C: Spectra}

The Hanning-smoothed spectra of the detected spectral lines are presented in Figs. C.1-C.33. In each panel, the fit to the line is superimposed as a green line.
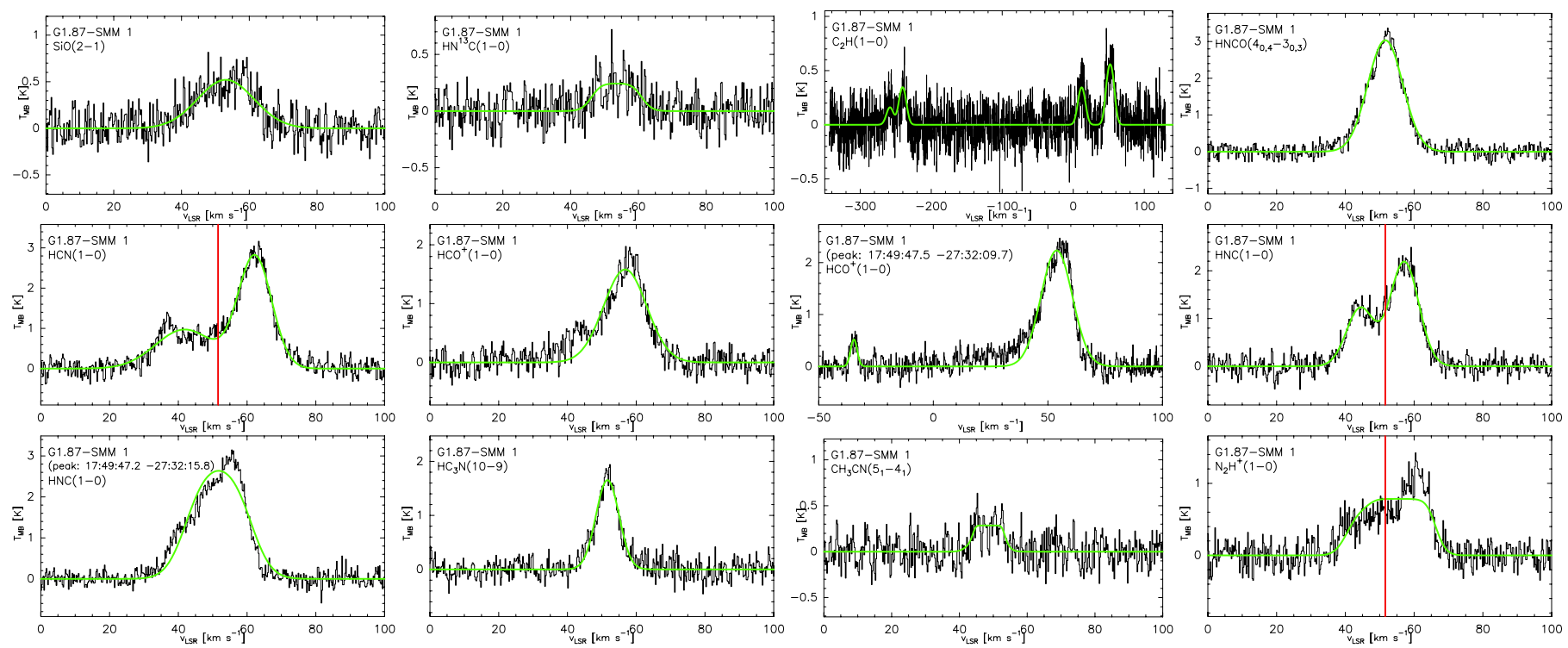

Fig. C.1. Hanning-smoothed spectra towards G1.87-SMM 1. The single-Gaussian and hf structure fits are shown with green lines. The vertical red line plotted on double-peaked profiles indicates the radial velocity of the optically thin $\mathrm{HC}_{3} \mathrm{~N}$ line. The velocity range is wider in the $\mathrm{C}_{2} \mathrm{H}$ and $\mathrm{HCO}^{+}$(line emission peak) spectra than in the other panels to show all the detected lines (the additional velocity component is included for the latter).
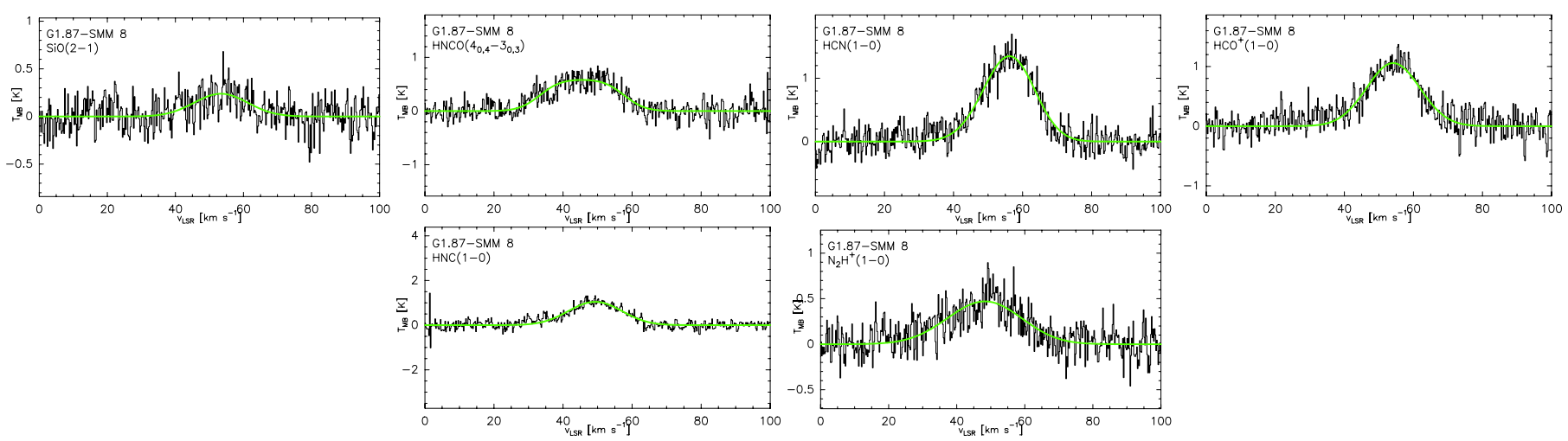

Fig. C.2. Same as Fig. C.1 but towards G1.87-SMM 8.
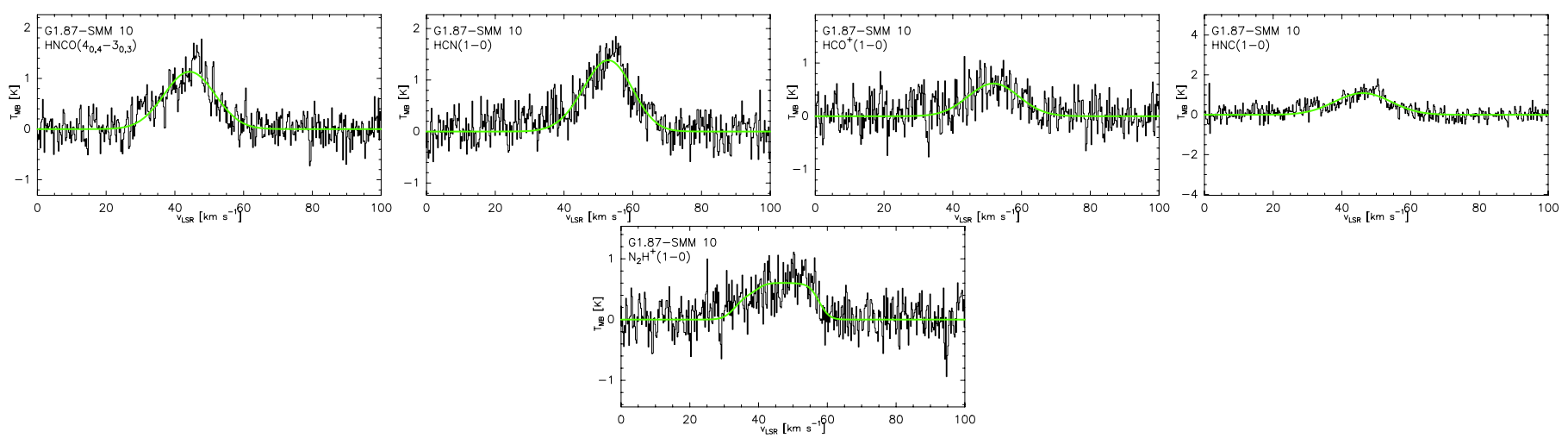

Fig. C.3. Same as Fig. C.1 but towards G1.87-SMM 10. 
O. Miettinen: A MALT90 chemical study of clumps within IRDCs
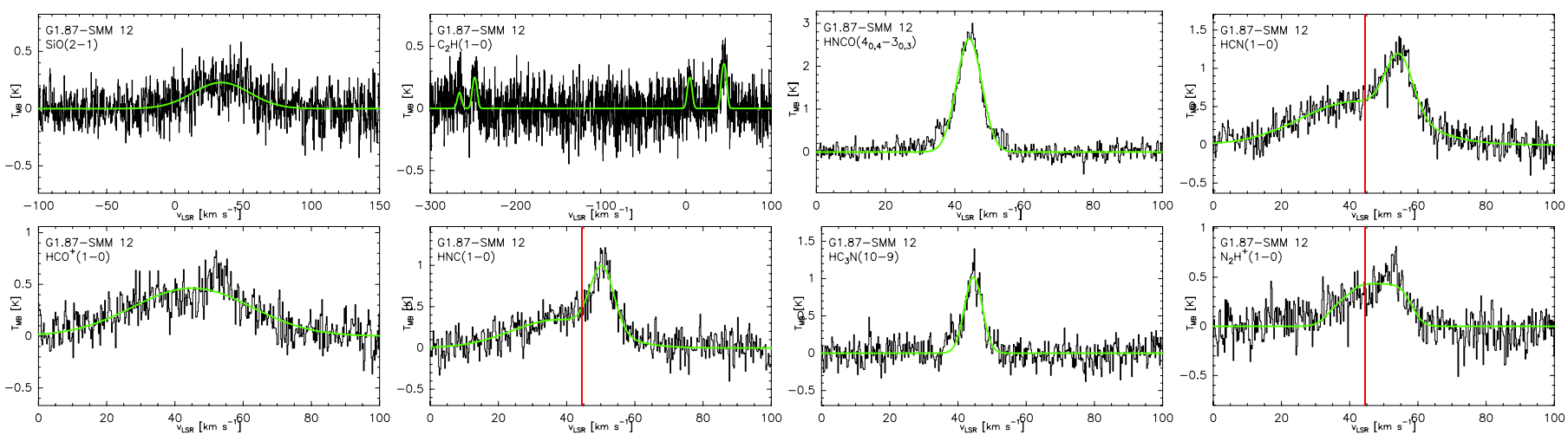

Fig. C.4. Same as Fig. C.1 but towards G1.87-SMM 12. We note that the velocity range for the $\mathrm{SiO}$ and $\mathrm{C}_{2} \mathrm{H}$ spectra is wider for illustrative purposes. The vertical red line marks the radial velocity of the optically thin $\mathrm{HC}_{3} \mathrm{~N}$ line.
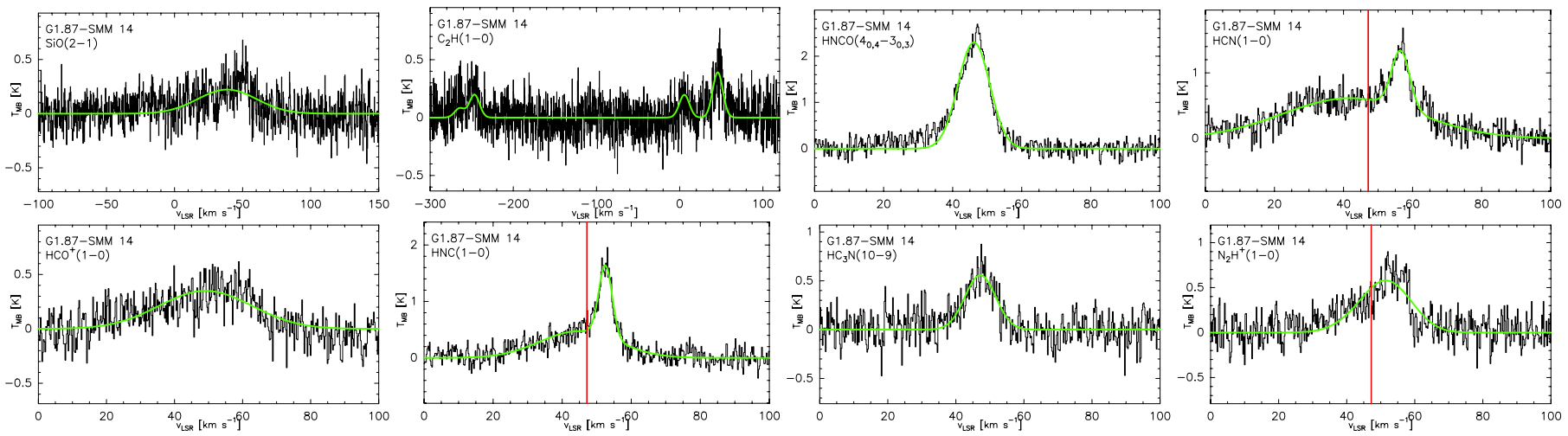

Fig. C.5. Same as Fig. C.1 but towards G1.87-SMM 14. We note that the velocity range for the $\mathrm{SiO}$ and $\mathrm{C}_{2} \mathrm{H}$ spectra is wider for illustrative purposes. The vertical red line marks the radial velocity of the optically thin $\mathrm{HC}_{3} \mathrm{~N}$ line.
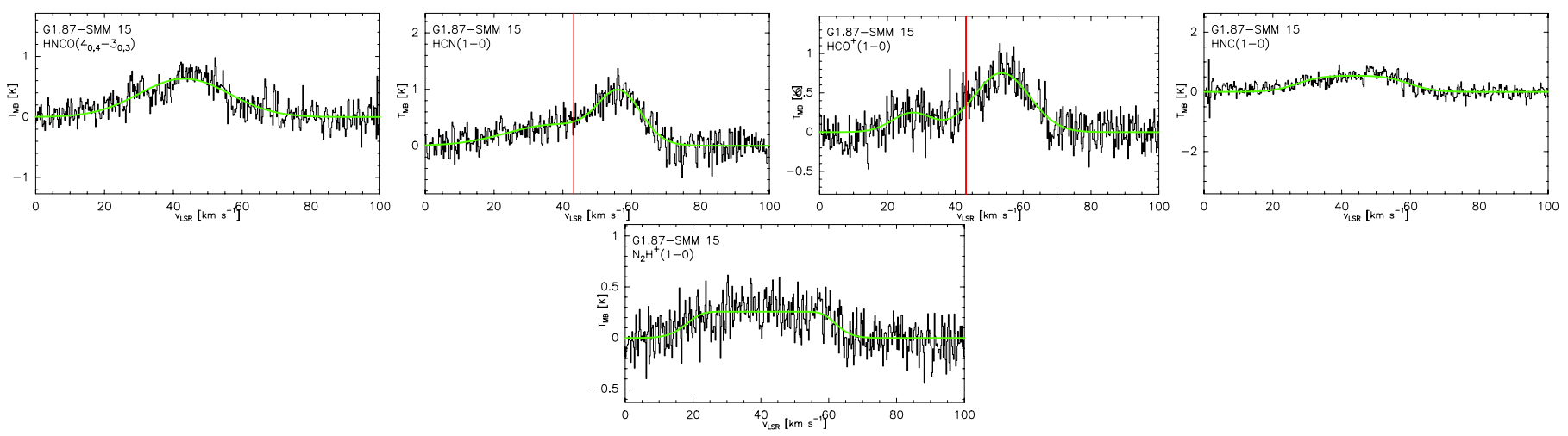

Fig. C.6. Same as Fig. C.1 but towards G1.87-SMM 15. The vertical red line indicates the radial velocity of the optically thin HNCO line.
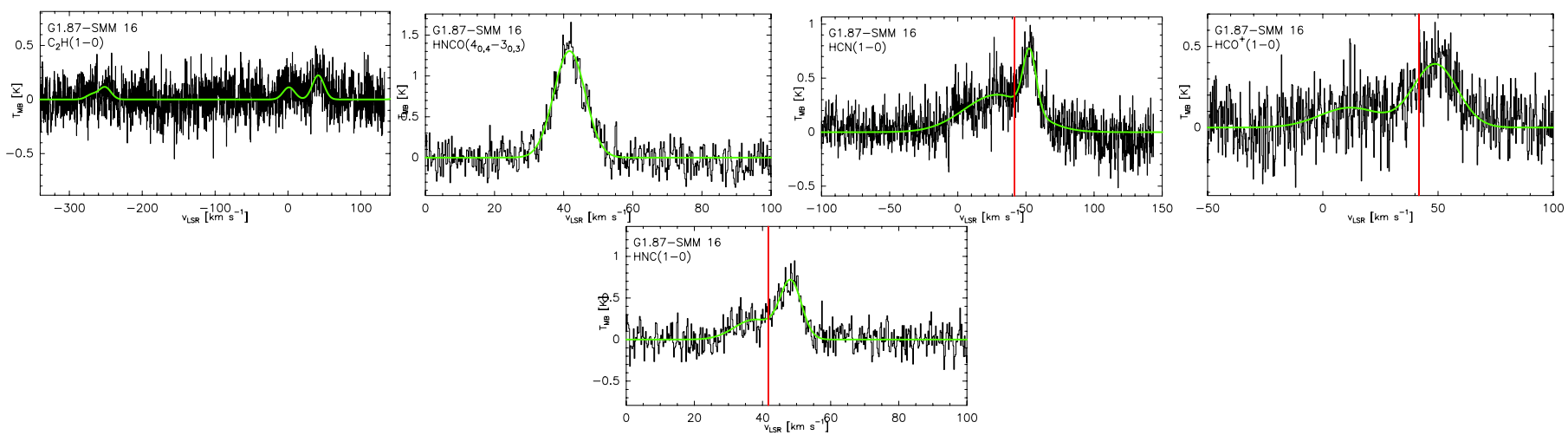

Fig. C.7. Same as Fig. C.1 but towards G1.87-SMM 16. In some of the spectra, the velocity range shown is wider for illustrative purposes. The vertical red line mark the velocity of the HNCO line. 
A\&A 562, A3 (2014)
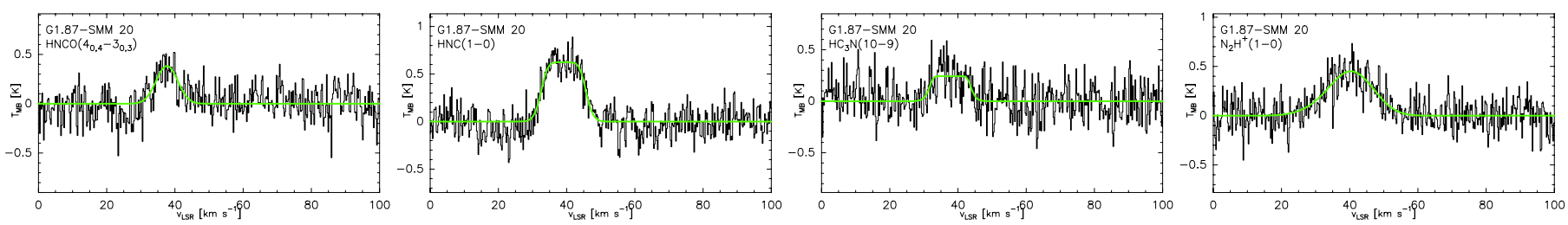

Fig. C.8. Same as Fig. C.1 but towards G1.87-SMM 20.
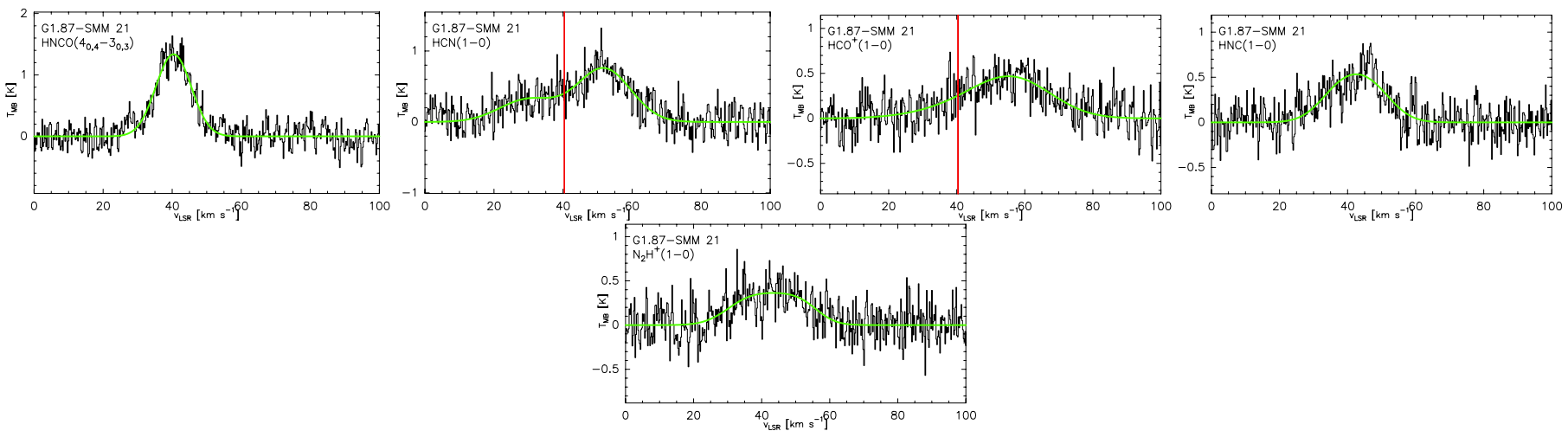

Fig. C.9. Same as Fig. C.1 but towards G1.87-SMM 21. The vertical red line indicates the radial velocity of the HNCO line.
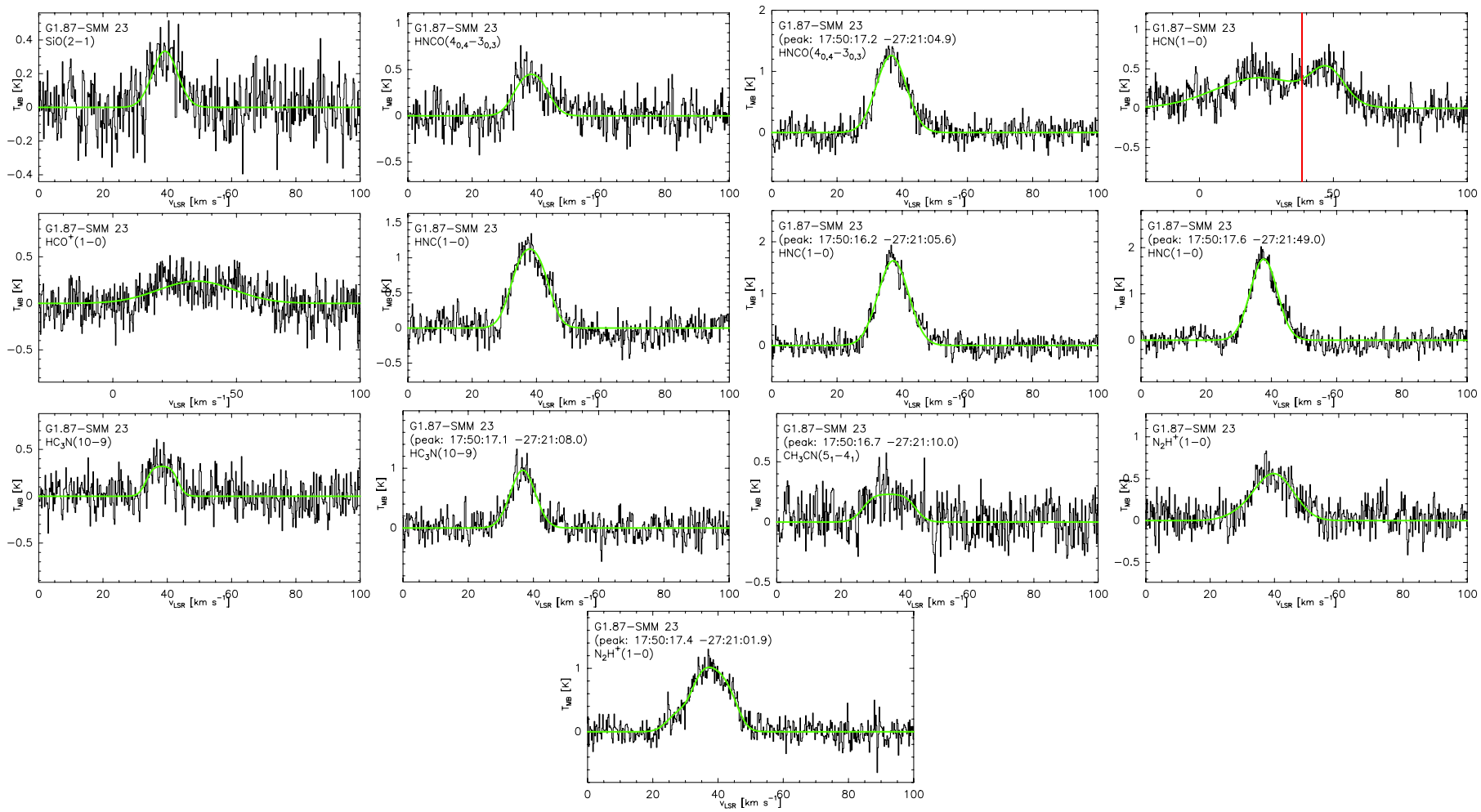

Fig. C.10. Same as Fig. C.1 but towards G1.87-SMM 23. We note that the velocity range for the $\mathrm{HCN}$ and $\mathrm{HCO}^{+}$spectra is wider for illustrative purposes. The vertical red line indicates the radial velocity of the optically thin $\mathrm{HC}_{3} \mathrm{~N}$ line. 
O. Miettinen: A MALT90 chemical study of clumps within IRDCs
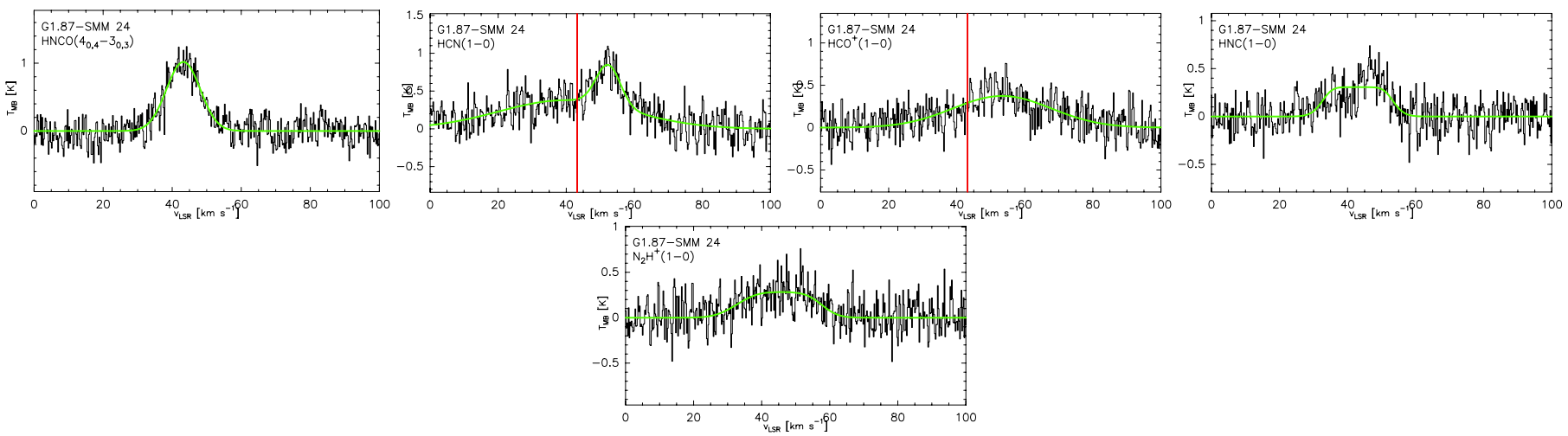

Fig. C.11. Same as Fig. C.1 but towards G1.87-SMM 24. The vertical red line indicates the radial velocity of the HNCO line.
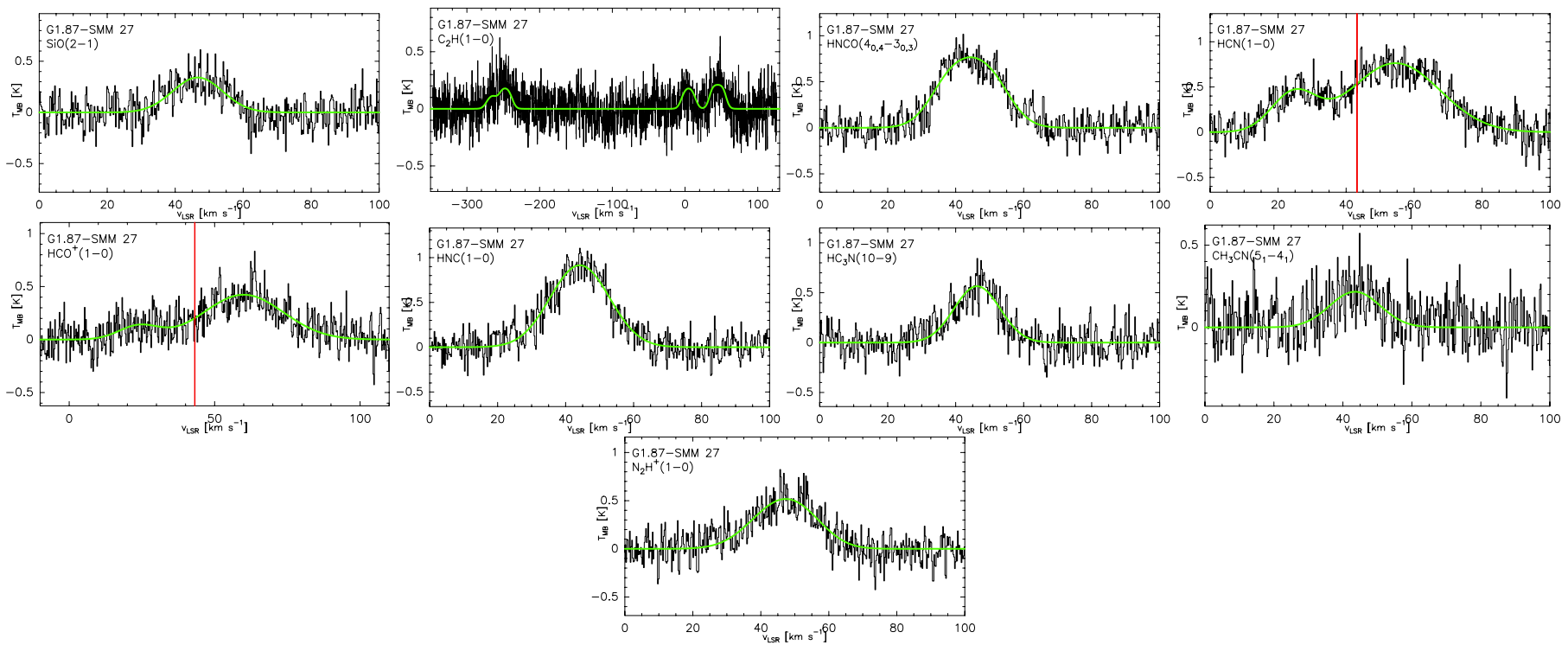

Fig. C.12. Same as Fig. C.1 but towards G1.87-SMM 27. The velocity range shown is wider for the $\mathrm{C}_{2} \mathrm{H}$ and $\mathrm{HCO}^{+}$spectra. The vertical red line indicates the radial velocity of the optically thin $\mathrm{HNCO}$ line.
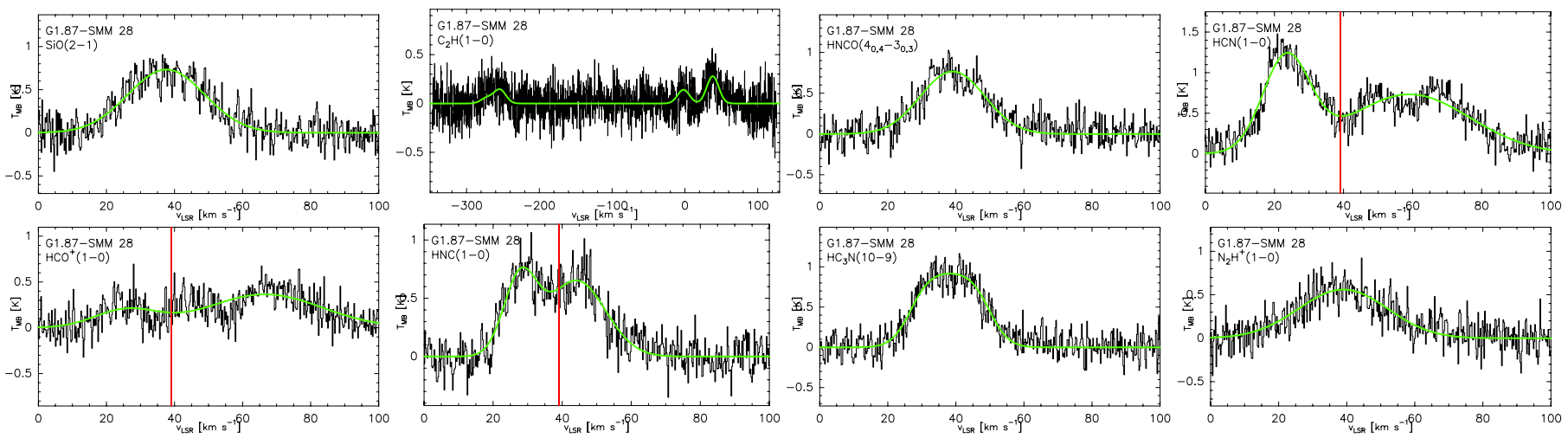

Fig. C.13. Same as Fig. C.1 but towards G1.87-SMM 28. The velocity range shown is wider for the $\mathrm{C}_{2} \mathrm{H}$ spectrum. The vertical red line indicates the radial velocity of the optically thin HNCO line. 
A\&A 562, A3 (2014)

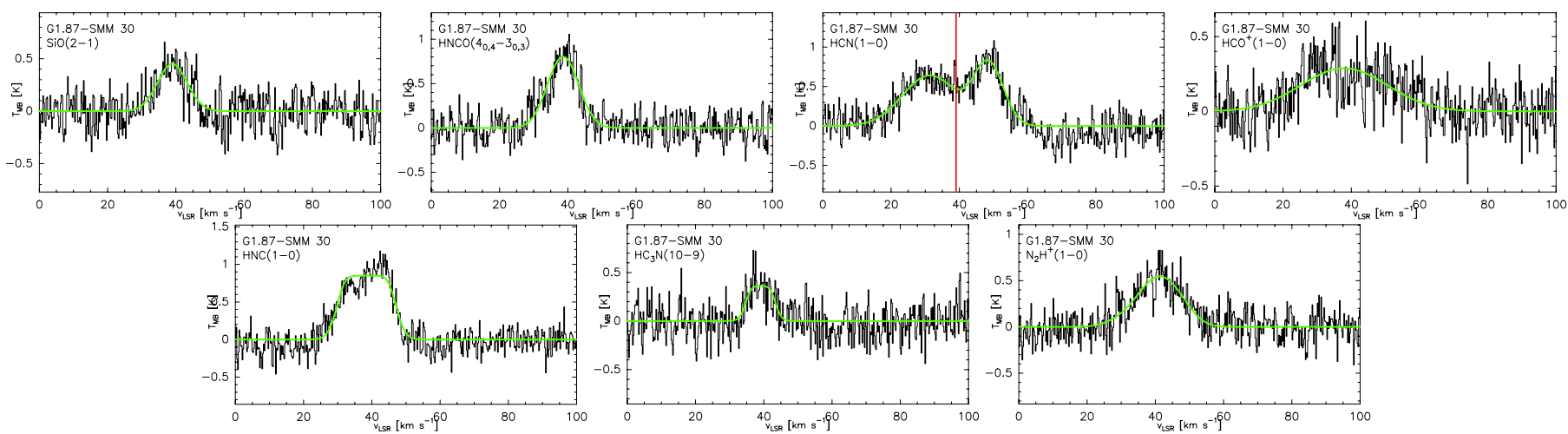

Fig. C.14. Same as Fig. C.1 but towards G1.87-SMM 30. The vertical red line indicates the radial velocity of the optically thin $\mathrm{HC}_{3} \mathrm{~N}$ line.

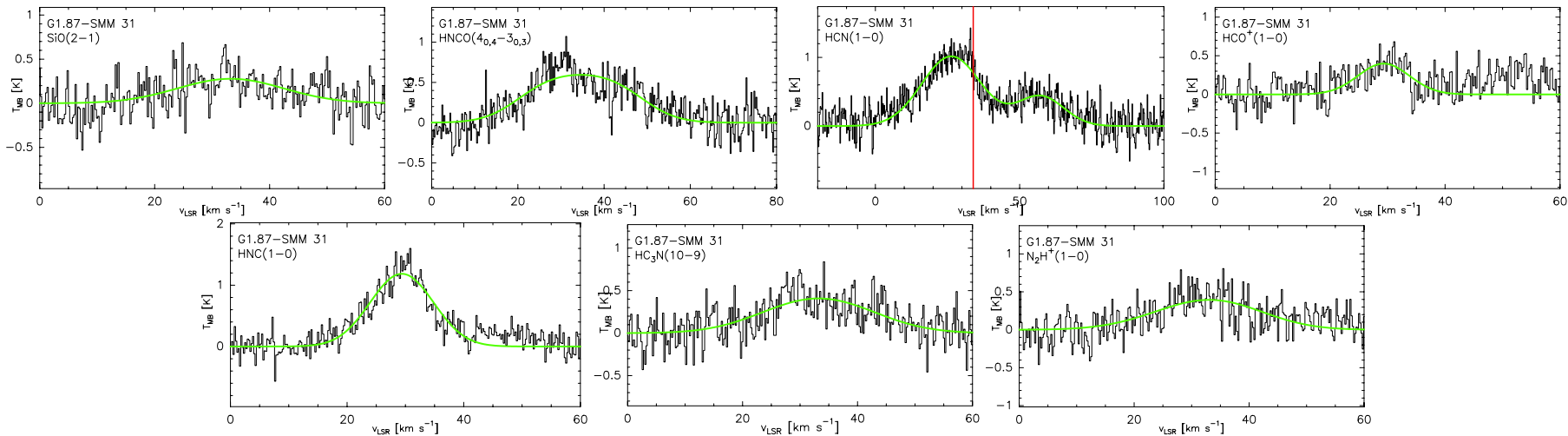

Fig. C.15. Same as Fig. C.1 but towards G1.87-SMM 31. The velocity range is wider for the HCN spectrum. The vertical red line indicates the radial velocity of the optically thin $\mathrm{HNCO}$ line.

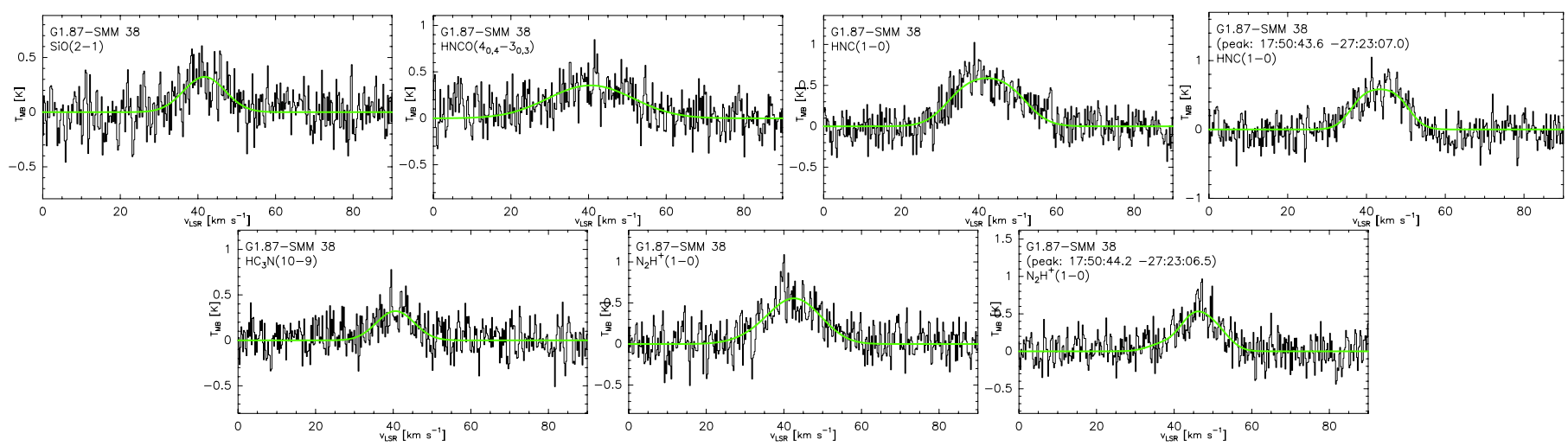

Fig. C.16. Same as Fig. C.1 but towards G1.87-SMM 38.
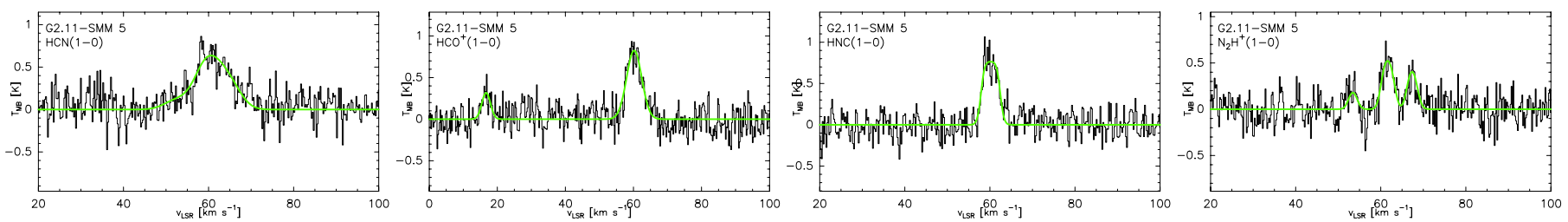

Fig. C.17. Same as Fig. C.1 but towards G2.11-SMM 5. A wider velocity range for the $\mathrm{HCO}^{+}$spectrum is shown because of the additional velocity component. 
O. Miettinen: A MALT90 chemical study of clumps within IRDCs
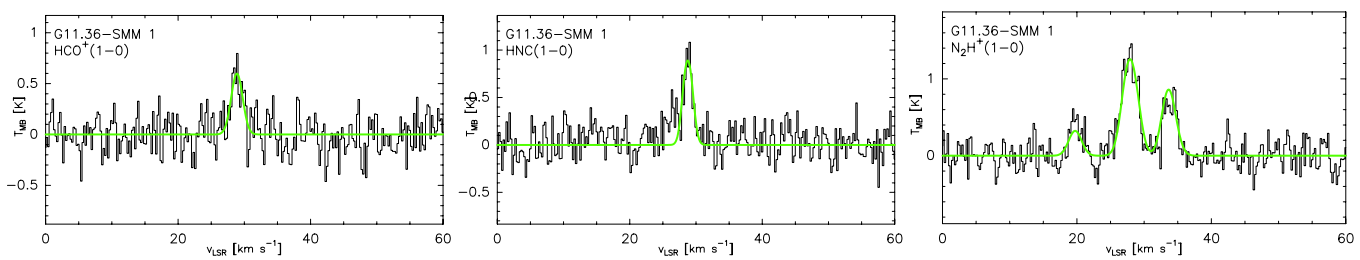

Fig. C.18. Same as Fig. C.1 but towards G11.36-SMM 1.
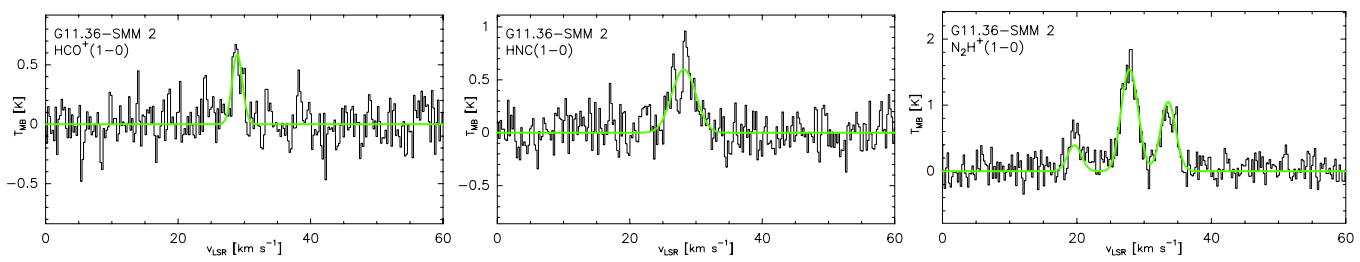

Fig. C.19. Same as Fig. C.1 but towards G11.36-SMM 2.
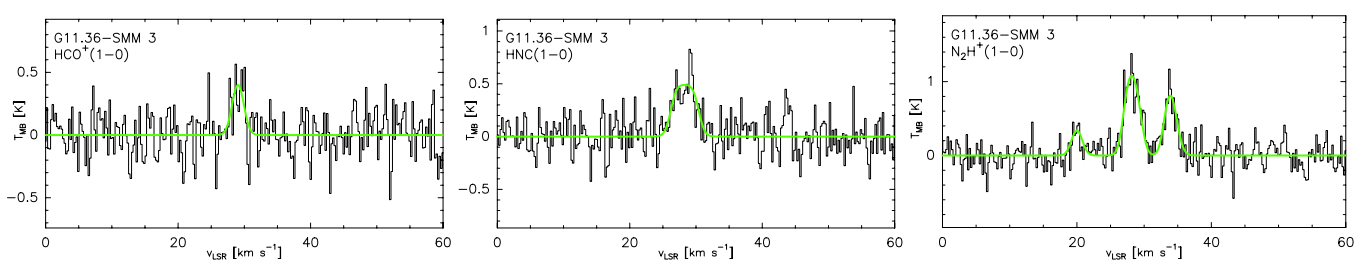

Fig. C.20. Same as Fig. C.1 but towards G11.36-SMM 3.

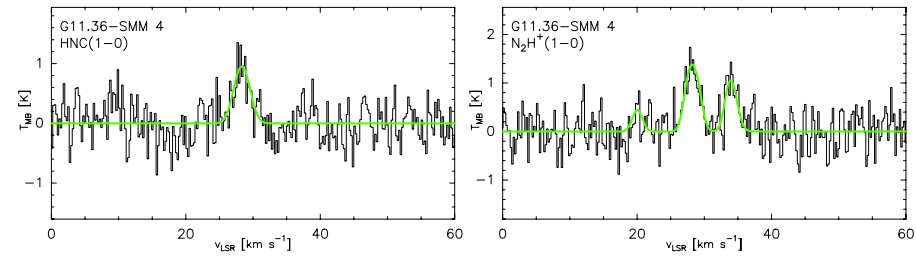

Fig. C.21. Same as Fig. C.1 but towards G11.36-SMM 4.
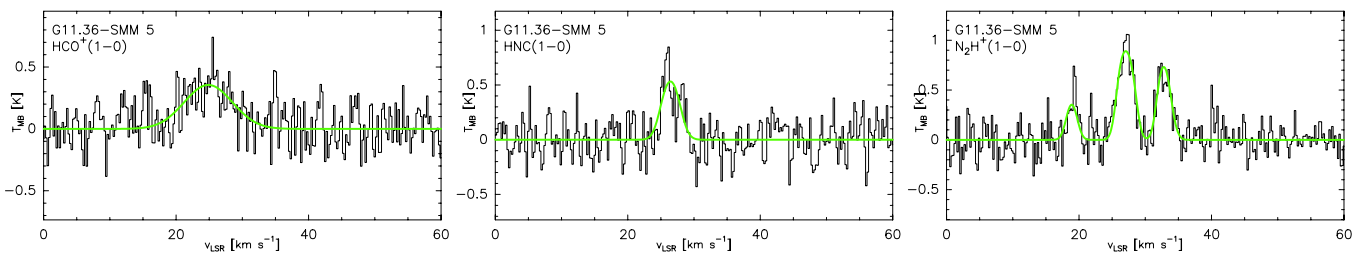

Fig. C.22. Same as Fig. C.1 but towards G11.36-SMM 5.
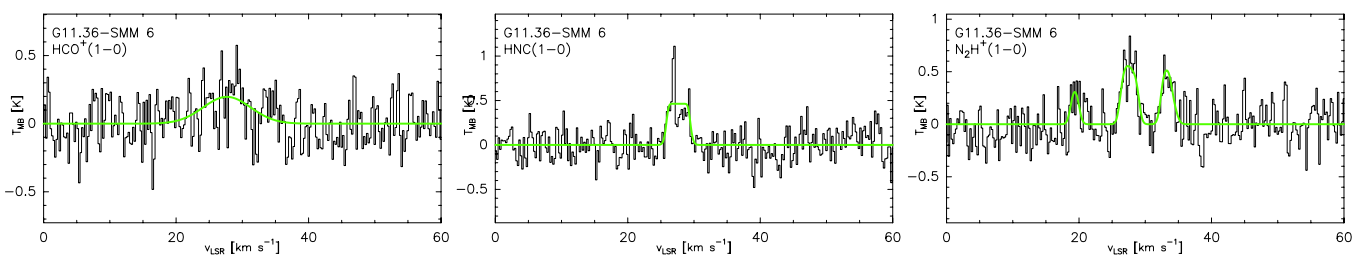

Fig. C.23. Same as Fig. C.1 but towards G11.36-SMM 6.
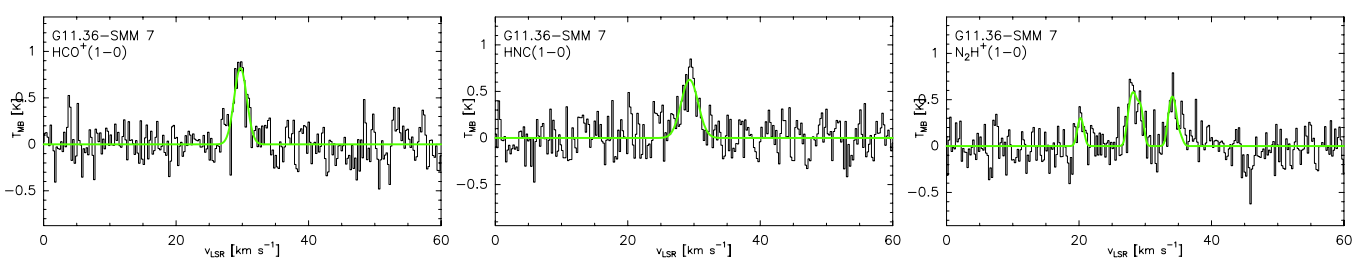

Fig. C.24. Same as Fig. C.1 but towards G11.36-SMM 7. 
A\&A 562, A3 (2014)
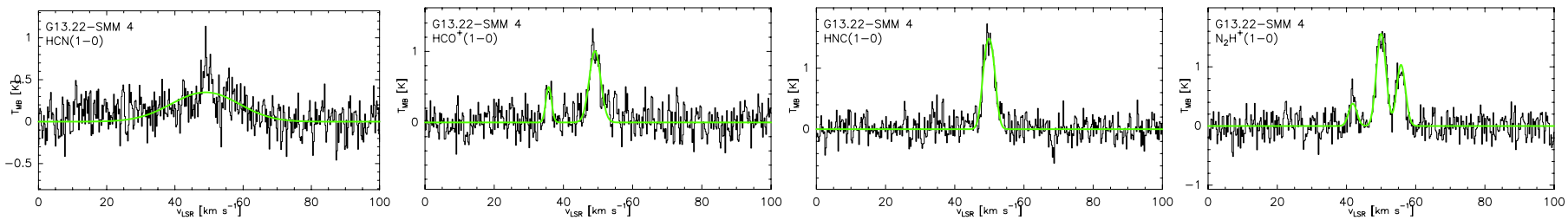

Fig. C.25. Same as Fig. C.1 but towards G13.22-SMM 4. There is an additional velocity component in the $\mathrm{HCO}^{+}$spectrum.
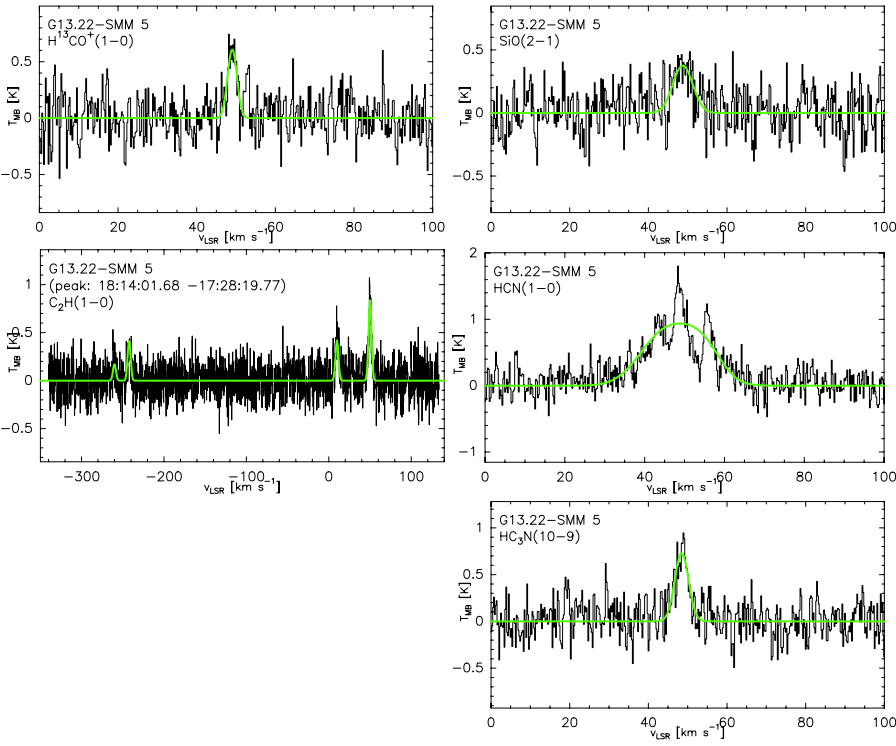
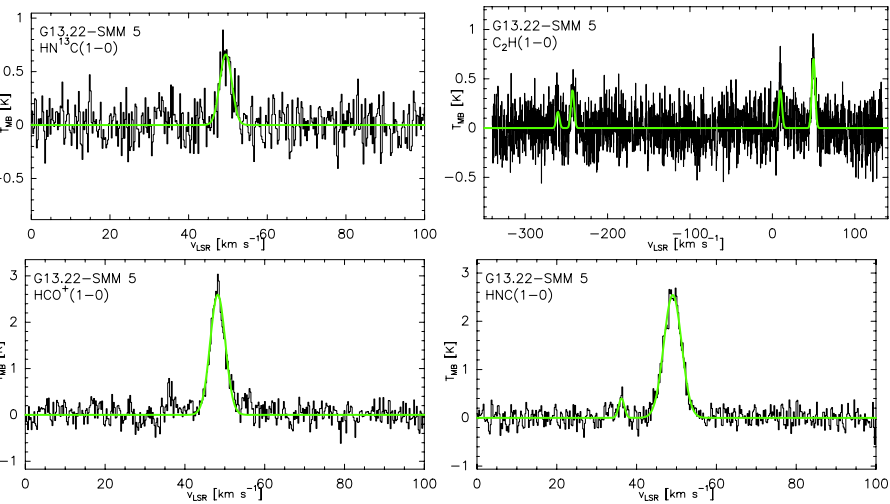

Fig. C.26. Same as Fig. C.1 but towards G13.22-SMM 5. The velocity range is wider for the two $\mathrm{C}_{2} \mathrm{H}$ spectra.
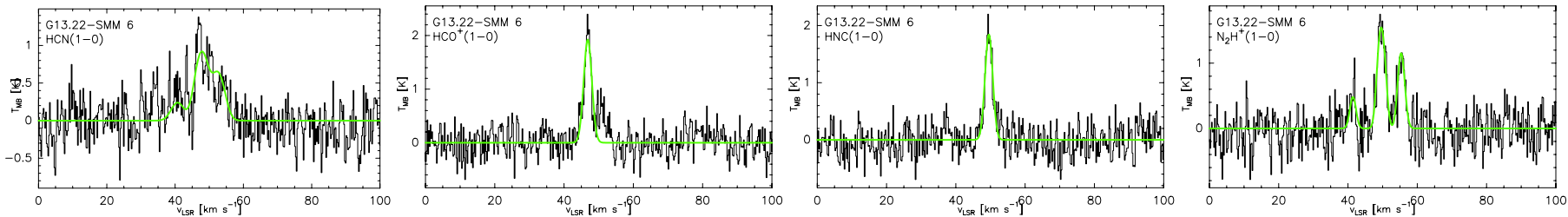

Fig. C.27. Same as Fig. C.1 but towards G13.22-SMM 6.
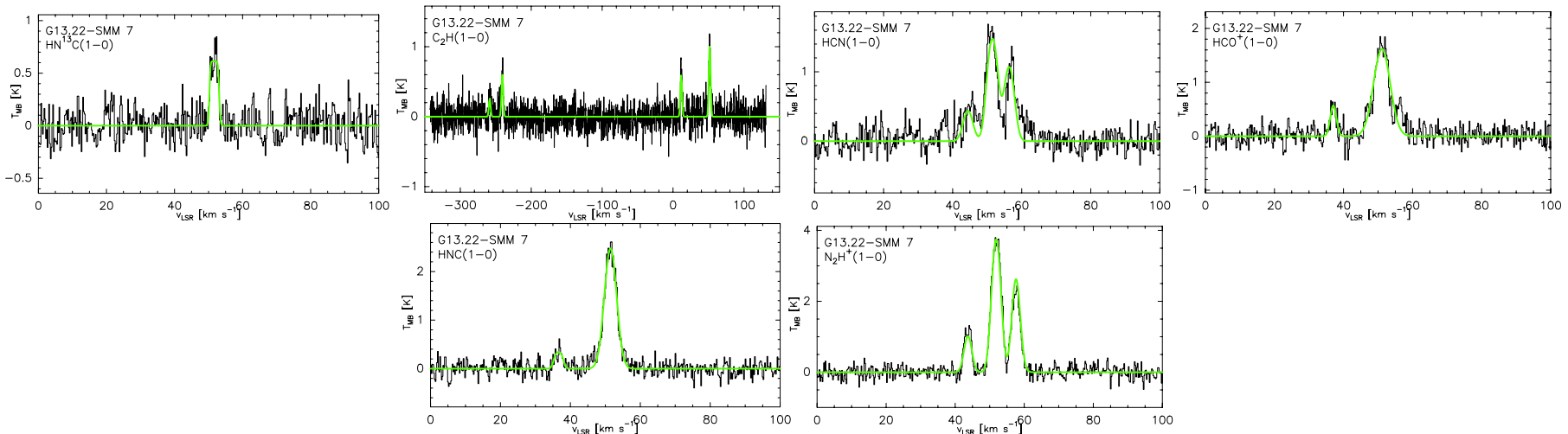

Fig. C.28. Same as Fig. C.1 but towards G13.22-SMM 7. The $\mathrm{C}_{2} \mathrm{H}$ spectrum has a wider velocity range. Two velocity components are seen in the $\mathrm{HCO}^{+}$and $\mathrm{HNC}$ spectra. 
O. Miettinen: A MALT90 chemical study of clumps within IRDCs
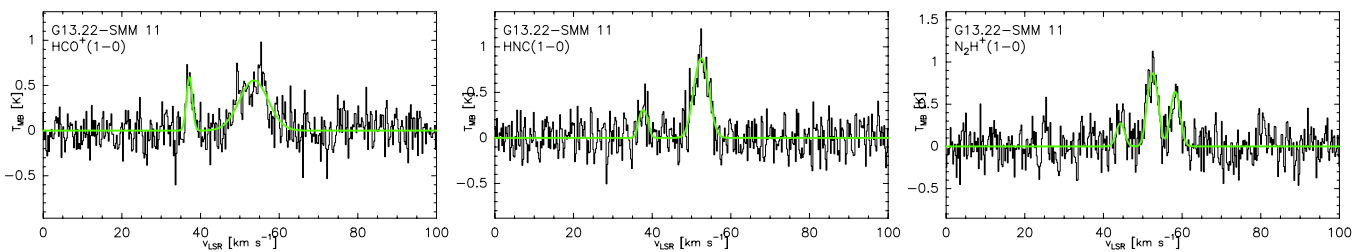

Fig. C.29. Same as Fig. C.1 but towards G13.22-SMM 11. Two velocity components are seen in the $\mathrm{HCO}^{+}$and $\mathrm{HNC}_{\text {spectra. }}$
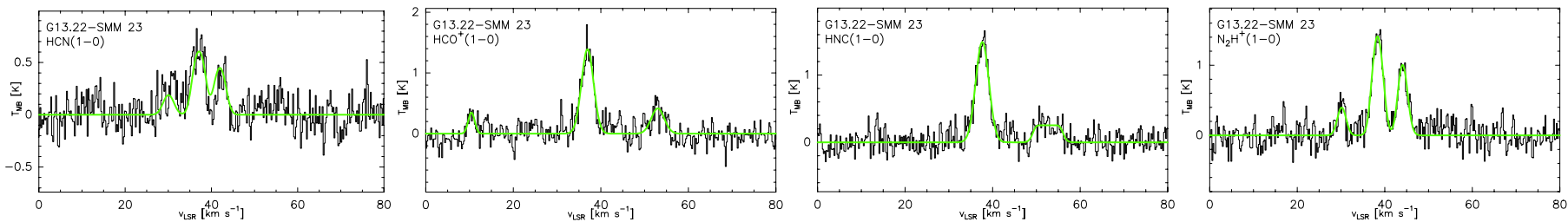

Fig. C.30. Same as Fig. C.1 but towards G13.22-SMM 23. Three velocity components are seen in the $\mathrm{HCO}^{+}$spectrum, while two are visible in the HNC spectrum.
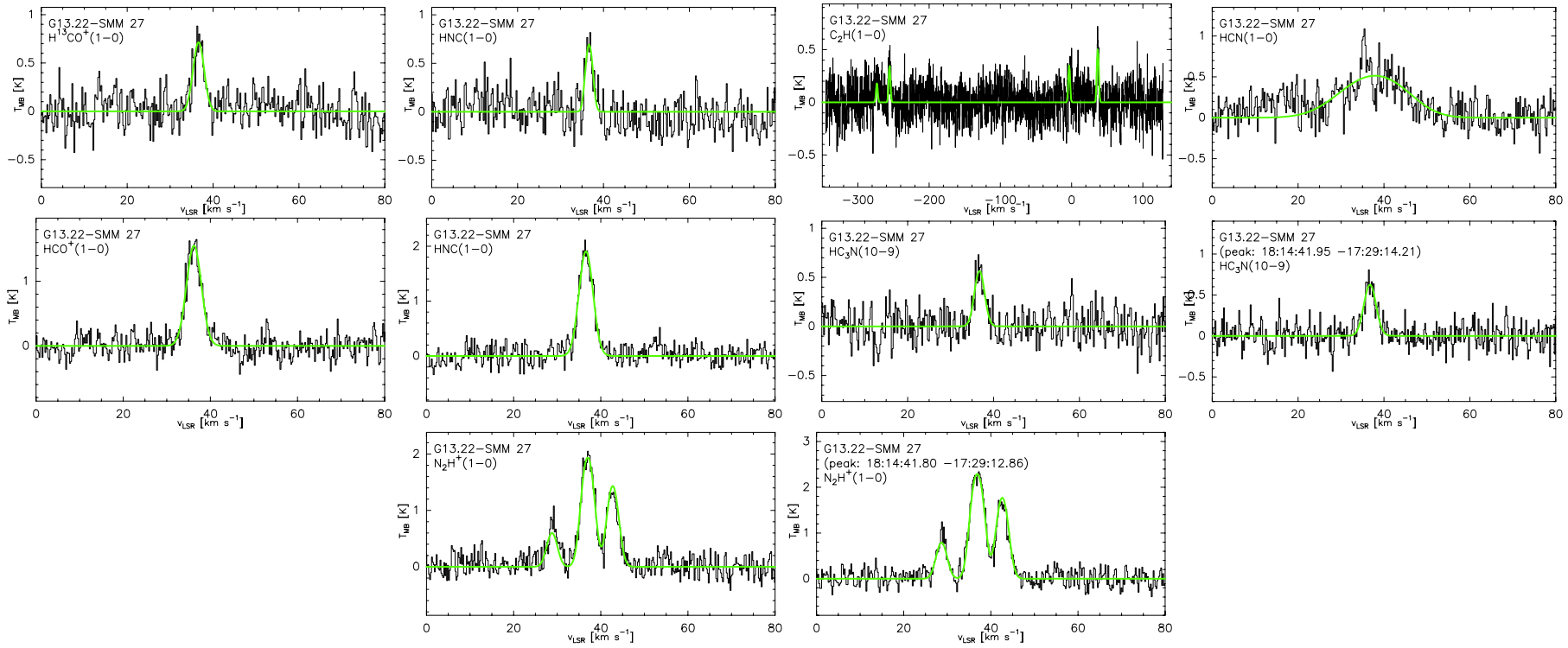

Fig. C.31. Same as Fig. C.1 but towards G13.22-SMM 27. The velocity range in the $\mathrm{C}_{2} \mathrm{H}$ spectrum is wider.
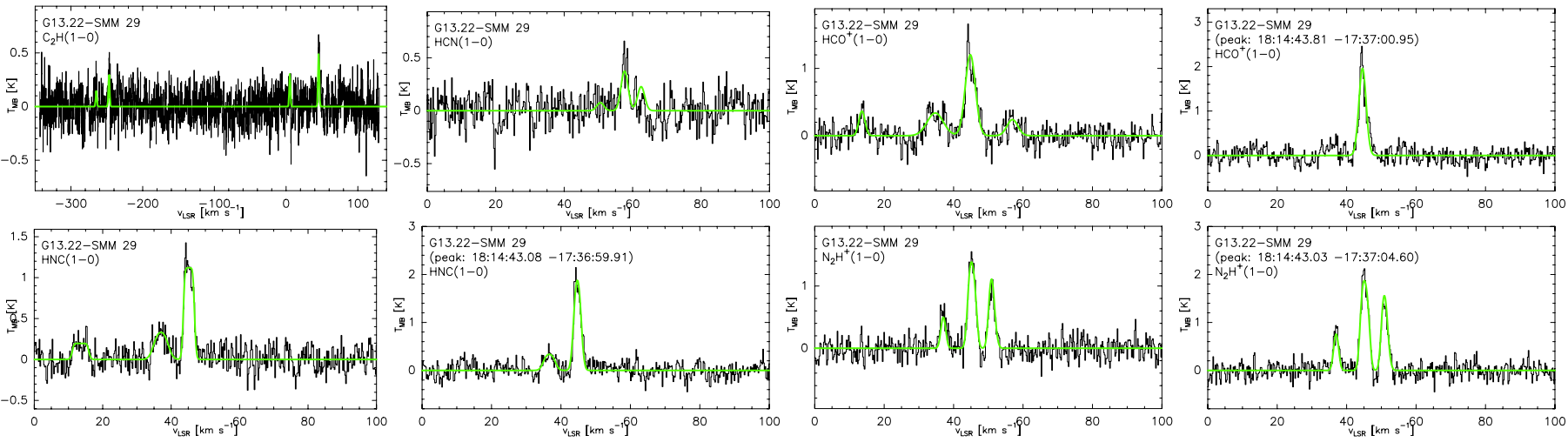

Fig. C.32. Same as Fig. C.1 but towards G13.22-SMM 29. The velocity range in the $\mathrm{C}_{2} \mathrm{H}$ spectrum is wider. Four velocity components are seen in the $\mathrm{HCO}^{+}$spectrum, three in the HNC spectrum towards the LABOCA peak, and two in the HNC spectrum towards the line emission peak. 
A\&A 562, A3 (2014)

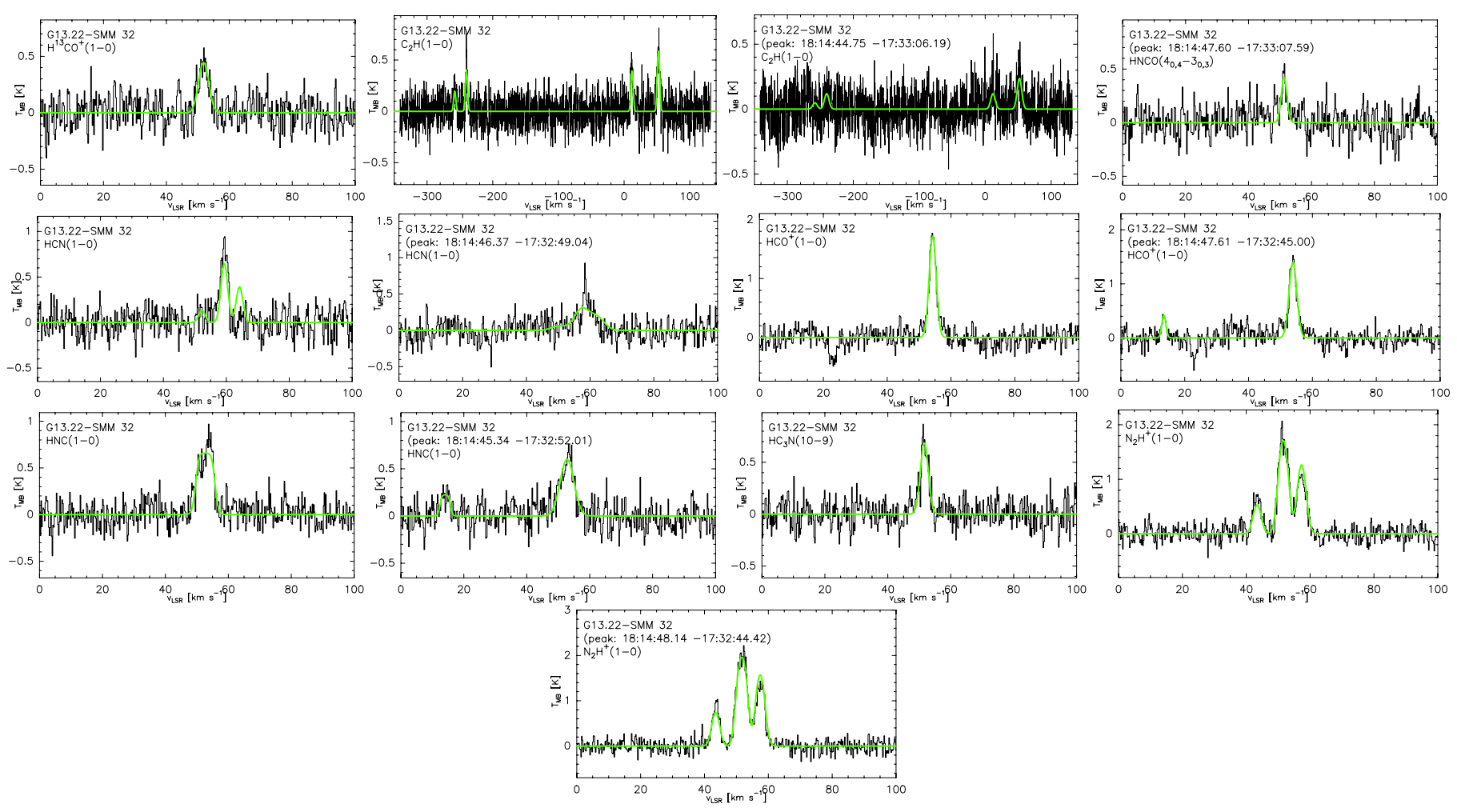

Fig. C.33. Same as Fig. C.1 but towards G13.22-SMM 32. The velocity range for the $\mathrm{C}_{2} \mathrm{H}$ spectra is wider. Two velocity components are detected in the $\mathrm{HCO}^{+}$and $\mathrm{HNC}$ spectra towards the line emission peaks. 